Linköping Studies in Science and Technology, Dissertations.

No. 977

\title{
Uplink Load in CDMA Cellular Radio Systems
}

\section{Erik Geijer Lundin}

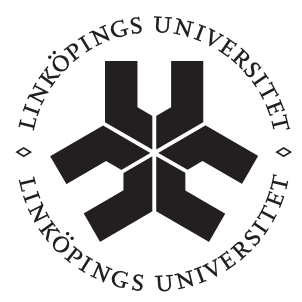

Department of Electrical Engineering

Linköpings universitet, SE-581 83 Linköping, Sweden

Linköping 2005 


\section{Uplink Load in CDMA Cellular Radio Systems}

(c) 2005 Erik Geijer Lundin

$$
\begin{gathered}
\text { geijerdisy.liu.se } \\
\text { www. control.isy.liu.se } \\
\text { Division of Automatic Control }
\end{gathered}
$$

Department of Electrical Engineering

Linköpings universitet

SE-581 83 Linköping

Sweden

ISBN 91-85457-49-3 ISSN 0345-7524

Printed by LiU-Tryck, Linköping, Sweden 2005 
To my sister and pärons! 



\section{Abstract}

The uplink of code division multiple access (CDMA) cellular radio systems is often interference limited. The interference originates from many users whose transmission powers are not observable for the system. This thesis introduces uplink load and applies means of explicitly considering the users' radio environment when approximating and controlling the load.

A desirable property of all cellular radio systems is uplink feasibility, i.e., existence of finite user transmission powers to support the allocated services. Uplink load can be considered as a measure of how far from infeasibility the system is. The performed characterization of uplink load lead to two concrete definitions related to the amount of received and transmitted power, respectively.

An important part of the total load is the intercell load which is caused by users connected to neighboring base stations. If not carefully handled, the intercell load can jeopardize uplink feasibility. Conversely, knowledge of a lower intercell load can be used to increase the resource assignments. A common denominator in all the work in this thesis is that the intercell load is explicitly considered.

When approximating uplink load, a centralized approach is adopted to study information gathered in several base stations. This yields good approximations of the average load. However, centralized approximations can not detect momentarily peaks in the load. A number of resource allocation algorithms making control decisions in the local base stations are proposed based on experience from characterizing uplink load. As the algorithms study the intercell load, yet without measuring the interference power, they are robust in the sense that they will never assign resources yielding an infeasible system.

A straightforward way of controlling the uplink load is to use measurements of the received interference power. This approach, just as the proposed load approximations, can gain from knowing the background noise power. The same framework used for designing robust resource allocation algorithms, is also used for estimating the background noise power. 


\section{Acknowledgments}

So these are the words of this thesis that will be read by the most people. The probability of me forgetting someone is certainly high, but I'll give it a go anyway.

First of all, the man who I got to know when he helped me every Tuesday, the whole day that is, during my master thesis, Dr. Fredrik Gunnarsson. Since then, he has excellently guided me through my time as a PhD student, without cutting down on those $20 \%$ of the work week. I am forever grateful for giving me exactly enough help to develop both my self and research results and never turning me down even when disturbing him at the Ericsson office. I don't know how many times he has read half done work, which I claimed was ready for publishing.

Since I never moved out of the office room I was first placed in, I have had the pleasure of having Professor Fredrik Gustafsson next door ever since I joined the automatic control group. This has made it easy for me to take advantage of his intuition and calm. He listens, even though it often does not look like it, and then proposes a simple solution to my, often poorly formulated, question.

I like to take the opportunity to thank Professor Lennart Ljung for creating a good working atmosphere through good leadership. I also owe Ulla Salaneck a thank you for cheerfully making all those administrative tasks work out smoothly.

The thesis has been proof read by Dr. Ragnar Wallin, Dr. Rickard Karlsson and David Törnqvist. Thank you for trying to make it readable even for those not already ensnared into the work. Gustaf Hendeby usually appears in acknowledgments for his $\mathrm{T}_{\mathrm{E}} \mathrm{X}$ skills. I would like to thank him for more than just helping with the practical stuff. He has been a good friend and ( $\mathrm{HT}_{\mathrm{E}} \mathrm{X}$-) companion during long weekends at the office. Without him, this thesis would not have happened as soon as it did.

Unfortunately, I will probably leave this place without repaying all the help I have received from people in the automatic control group. As has been concluded by many people before me, the group has a good atmosphere and everyone is willing to help. A thank you goes out to all the people in the group. There are some people who I have bothered also after office hours. These include Jonas Gillberg, who has been a good friend in tough situations, and Daniel Axehill with whom I have had rewarding discussions and received many good advice about boats of all kinds (they certainly helped during those long tedious periods away from the sea).

Dr. Gunnar Bark, Dr. Niclas Wiberg and Dr. Eva Englund are some of the people working in the group at Ericsson, LinLab, that deserve a big thank you for being an endless source of inspiration and sharing their huge knowledge within radio resource management.

This project has been supported financially by the VINNOVA Center of Excellence ISIS and the SSF graduate school ECSEL, which are gratefully acknowledged. Ericsson has provided both financial support and access to resources at their Linköping office which is much appreciated.

A big thank you goes out to Ludde, Eva and Karin for the support and hospitality they have shown without getting much in return. I would also like to thank Olle Berggren, the only child hood friend I have left in the Swedish royal capital, for always having a place in his fishing boat despite me usually calling first during the late Friday afternoon. 



\section{Contents}

1 Introduction 1

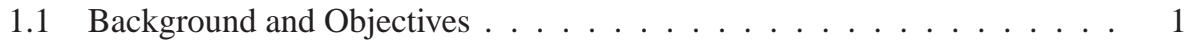

1.2 Thesis Outline . . . . . . . . . . . . . . . . . . . . 2

1.3 Contributions and Publications $\ldots \ldots \ldots \ldots \ldots$

2 Extended Summary $\quad 7$

2.1 Cellular Radio Communication . . . . . . . . . . . . . . . . . . . 7

2.1.1 System Model . . . . . . . . . . . . . . . . . . . 7

2.1.2 Assumptions and Scope of the Thesis . . . . . . . . . . . 9

2.2 Characterizing Uplink Load . . . . . . . . . . . . . . . . . . 10

2.3 Approximating Uplink Load . . . . . . . . . . . . . . . . . . 12

2.3.1 Derivation of Load Approximations . . . . . . . . . . . . 13

2.3.2 Error Sources . . . . . . . . . . . . . . . . . . . . . . . . . . 14

2.3.3 Evaluation . . . . . . . . . . . . . . . . . 14

2.4 Analyzing Uplink Load . . . . . . . . . . . . . . . . . . . . . 15

2.5 Controlling Uplink Load . . . . . . . . . . . . . . . . . . . . . 16

2.6 Feasibility versus Coverage . . . . . . . . . . . . . . . . . . . . 19

2.7 Filtering and Estimating Uplink Load . . . . . . . . . . . . . 20

2.7.1 Noise Rise Relative Load Filtering . . . . . . . . . . . . . . . 20

2.7.2 Background Noise Power Estimation _ . . . . . . . . . 21

3 Cellular Radio Communication 25

3.1 Radio Wave Propagation . . . . . . . . . . . . . . . . . . . . 26

3.2 Radio Communication Systems . . . . . . . . . . . . . . . . 28

3.3 Multiple Access . . . . . . . . . . . . . . . . . . . . . . . . . . . . 29

3.3.1 Orthogonal Signals . . . . . . . . . . . . . . . . . . . . . . . . . 29

3.3.2 Nonorthogonal Signals . . . . . . . . . . . . . . . . . . . . 29 
3.4 Cellular Radio Networks . . . . . . . . . . . . . . . . . . . . 30

3.5 System Performance . . . . . . . . . . . . . . . . . . . . 33

3.6 Radio Resource Management Algorithms . . . . . . . . . . . . . . . . 34

3.6.1 Power Control . . . . . . . . . . . . . . . . . . 34

3.6 .2 Load Control . . . . . . . . . . . . . . . . . . 35

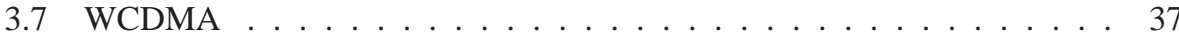

4 Characterizing Uplink Load 39

4.1 System Load and Capacity . . . . . . . . . . . . . . . . . . . . . . . . . 40

4.2 Decentralized Load . . . . . . . . . . . . . . . . . . . . . . . . 43

4.2.1 Intercell-to-Intracell-Interference Factor . . . . . . . . . . . . . . 44

4.2.2 Interference Power Measurements . . . . . . . . . . . . . . . 45

4.3 Centralized Load . . . . . . . . . . . . . . . . . . . . . . . . . . . . . . . . . . . . . . . . .

4.3.1 Feasibility Relative Load . . . . . . . . . . . . . . . . . . 46

4.3.2 Convergence of Power Control Algorithms . . . . . . . . . . . . 48

4.3.3 Link Based Estimates . . . . . . . . . . . . . . . . . 50

4.4 Discussion . . . . . . . . . . . . . . . . 52

5 Approximating Uplink Load 53

5.1 Uplink Interference Power Expressions . . . . . . . . . . . . . . . . . 53

5.2 Uplink Load Expressions . . . . . . . . . . . . . . . . . . . 55

5.2.1 Methods for Solving Nonlinear Equations . . . . . . . . . . . . 56

5.2.2 Approximation I: Equal Interference Power In All Cells . . . . . 56

5.2.3 Approximation II: Equal Background Noise Power . . . . . . . . 58

5.2.4 Approximation III: Distributed Information . . . . . . . . . . . . 60

5.2 .5 Required Information . . . . . . . . . . . . . . . 61

5.3 Comparison of the Uplink Load Expressions . . . . . . . . . . . . . . . . 62

5.4 Sources of Estimation Errors . . . . . . . . . . . . . . . . . 63

5.4.1 Nonlinear Relation Between CIR and CTIR . . . . . . . . . . 63

5.4 .2 TX Increase . . . . . . . . . . . . . . . . . . . . . . 64

5.5 TX Increase Compensation . . . . . . . . . . . . . . . . . . . 65

5.6 Summary of Proposed Approximations and Compensation Methods . . . 67

5.7 Simulations . . . . . . . . . . . . . . . . . 68

5.7 .1 Simulation Setup . . . . . . . . . . . . . . . . 68

5.7.2 Measurement Report Frequency . . . . . . . . . . . . . . . . 68

5.7.3 One Radio Network Controller . . . . . . . . . . . . . . . . . . . 70

5.7.4 Several Radio Network Controllers . . . . . . . . . . . . . . 75

5.8 Summary ........................... 77

6 Analyzing Uplink Load 81

6.1 System Properties . . . . . . . . . . . . . . . . . . . . . 82

6.1 .1 Terminology .................... 82

6.1.2 Interference Power Expression . . . . . . . . . . . . . . . . . 83

6.2 System Load . . . . . . . . . . . . . . . . . . . . . . . . . . . . . . 84

6.2.1 System Noise Rise Relative Load . . . . . . . . . . . . . . . . . 84

6.2.2 Feasibility Relative Load . . . . . . . . . . . . . . . . . . . 84 
6.3 Estabilishing the Feasibility Relative Load . . . . . . . . . . . . . . . . 86

6.3.1 Connectivity One . . . . . . . . . . . . . . . 86

6.3 .2 Higher Connectivity . . . . . . . . . . . . . . . . . . . . . . . . . . . 89

6.4 Relative Load Comparisons . . . . . . . . . . . . . . . . . . . . . 90

6.4.1 Connectivity One . . . . . . . . . . . . . . . 90

6.4.2 Higher Connectivity . . . . . . . . . . . . . . . . . . . . 92

6.5 Convergence of Fix Point Iterations . . . . . . . . . . . . . . . . . . . . . . . . . . . 92

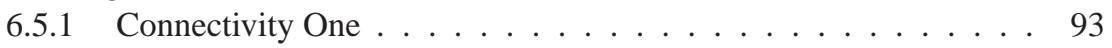

6.5.2 Higher Connectivity . . . . . . . . . . . . . . . . 93

6.6 Approximating Uplink Load in Practice . . . . . . . . . . . . . . . . . 93

6.7 A Simulation Example . . . . . . . . . . . . . . . . . . . . . . . . . . . . . . . . 93

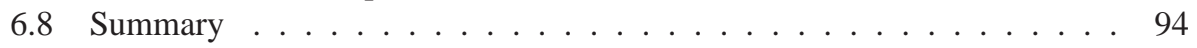

7 Controlling Uplink Load 97

7.1 Practical Centralized Resource Allocation . . . . . . . . . . . . . . . . . 98

7.2 Resource Control Approaches . . . . . . . . . . . . . . . . . . 100

7.3 Resource Control Algorithms . . . . . . . . . . . . . . . . . . 102

7.3.1 Centralized Robust Algorithm . . . . . . . . . . . . . . 103

7.3.2 Semi-Centralized Robust Algorithm . . . . . . . . . . . . . . 103

7.3.3 Decentralized Robust Algorithms . . . . . . . . . . . . . . . 107

7.3.4 Blind Algorithms . . . . . . . . . . . . . . . . . . . 110

7.4 Resource Control Evaluations . . . . . . . . . . . . . . . . . . 111

7.5 Inaccurate Background Noise Power Knowledge . . . . . . . . . . . . . 113

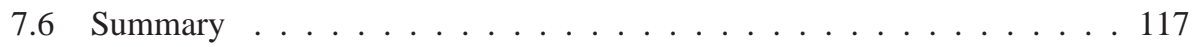

8 Feasibility versus Coverage 119

8.1 Unlimited Transmission Powers . . . . . . . . . . . . . . . . 120

8.1 .1 Two Users . . . . . . . . . . . . . . . . . . . 120

8.1.2 Several Users . . . . . . . . . . . . . . . . . . . . . . . . . . . . . . . . . . . . . . . . . . .

8.2 Limited Transmission Powers . . . . . . . . . . . . . . . . . . . . 121

8.2.1 Link Budget . . . . . . . . . . . . . . . . . . 121

8.2.2 Density Functions and Constants . . . . . . . . . . . . . . 122

8.2 .3 Simulations . . . . . . . . . . . . . . . 123

8.3 Maximum Load . . . . . . . . . . . . . . . . . . . . . . . . . . . . . . . . . . . . . . . . . . . . . . . .

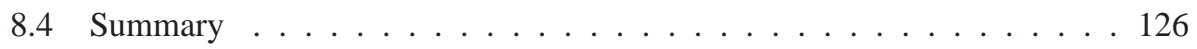

9 Filtering and Estimating Uplink Load 129

9.1 Adaptive Filtering Theory . . . . . . . . . . . . . . . . . . . . . 129

9.1.1 Kalman Filtering . . . . . . . . . . . . . . . . 130

9.1.2 Linearized Kalman Filtering . . . . . . . . . . . . . . . . . . 131

9.1.3 Extended Kalman Filtering . . . . . . . . . . . . . . . . . . . 132

9.1.4 Particle Filtering . . . . . . . . . . . . . . . . . . . . 132

9.1.5 Change Detection . . . . . . . . . . . . . . . . . . . . . . . . . . . . . . . .

9.2 Load Filtering . . . . . . . . . . . . . . . . . . . . . . . . . . . . . . . . . . . . . . . . . . . . .

9.2.1 Motivation . . . . . . . . . . . . . . . 135

9.2.2 Signal Model . . . . . . . . . . . . . . . . . 137 
9.2 .3 Design Choices . . . . . . . . . . . . . . . 138

9.2.4 Simulations . . . . . . . . . . . . . . . . . 140

9.3 Background Noise Power Estimation . . . . . . . . . . . . . . . . . . . 140

9.3.1 Connectivity One . . . . . . . . . . . . . . 142

9.3.2 Higher Connectivity . . . . . . . . . . . . . . . . 143

9.3.3 Estimation Limitations . . . . . . . . . . . . . . . . . . . . 144

9.3 .4 Simulations . . . . . . . . . . . . . . . . 146

9.3.5 Implementation Aspects . . . . . . . . . . . . . . . . . . 149

9.4 Summary . . . . . . . . . . . . . . . . . . . . . 149

10 Conclusions $\quad 151$

A Appendix 153

A.1 Cellular Radio System Simulator . . . . . . . . . . . . . . . . . 153

A.1.1 Models . . . . . . . . . . . . . . . . 153

A.1.2 Other Features . . . . . . . . . . . . . . . . . . 154

A.1.3 Simulator Utilization . . . . . . . . . . . . . . . . . . 154

A.2 Positive Matrices Theory . . . . . . . . . . . . . . . . . 154

A.3 Schur Complement . . . . . . . . . . . . . . . . . 155

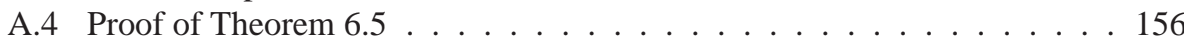

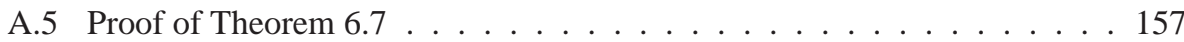

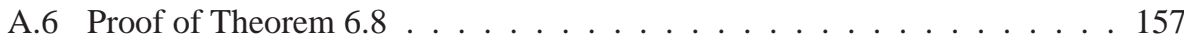

A.6.1 Preliminaries . . . . . . . . . . . . . 157

A.6.2 Proof of Theorem $6.8 \ldots \ldots \ldots 159$

A.6.3 Standard Interference Functions . . . . . . . . . . . . . 159

A.7 Proof of Lemma $7.2 \ldots \ldots$. . . . . . . . . . . . . . . . . 160

$\begin{array}{ll}\text { Bibliography } & 161\end{array}$

$\begin{array}{ll}\text { Index } & 169\end{array}$ 


\section{Notation}

\section{Symbols}

$B \quad$ Number of base stations in the system, 30

$G \quad$ Power gain matrix, 30

$I^{\text {other }}$ Intercell interference power, 43

$I^{\text {own }} \quad$ Intracell interference power, 43

$I^{\text {tot }} \quad$ Total received interference power, 31

$K \quad$ Link matrix, 82

$L \quad$ System matrix, 83

$L^{n r} \quad$ Noise rise relative load, 41

$L_{f} \quad$ Feasibility relative load, 85

$L_{s} \quad$ System noise rise relative load, 84

$M \quad$ Number of users in the entire system, 30

$N \quad$ Background noise power, 31

$Q \quad$ Covariance matrix, process noise, 130

$R \quad$ Covariance matrix, measurement noise, 130

$Z \quad$ Relative power gain matrix, 83

$\Lambda \quad$ Noise rise, 40

$\alpha \quad$ Propagation exponent, 26

$\alpha \quad$ Self interference factor, 32

$\bar{\lambda} \quad$ Eigenvalue with largest magnitude, 47

$\beta \quad$ Carrier-to-Total-Interference Ratio (CTIR), 31

$\beta^{\text {tgt }} \quad$ Target CTIR, 42

$\beta_{0} \quad$ Target CTIR in a single service system, 43

$\epsilon \quad$ Residual, 130

$\gamma \quad$ Carrier-to-Interference Ratio (CIR), 31

$\gamma^{\text {tgt }} \quad$ target CIR, 35 


$\begin{array}{ll}\hat{x}(t \mid \tau) & \text { Estimate of } x(t) \text { using data until time } \tau, 130 \\ \theta & \text { Background noise power ratio, } 114 \\ \underline{\lambda} & \text { Eigenvalue with smallest magnitude, } 90 \\ f & \text { Intercell-to-intracell-interference factor, } 44 \\ g & \text { Power gain, 26 } \\ p & \text { Transmission power, } 31 \\ q & \text { Shift operator, } q^{-1} x(t)=x(t-1), 27 \\ x_{0} & \text { Linearization point, working point, } 131\end{array}$

\section{Abbreviations}

CDMA Code Division Multiple Access, 29

CIR Carrier-to-Interference Ratio, 28

CTIR Carrier-to-Total-Interference Ratio, 31

CUSUM CUmulative SUM, 134

EKF Extended Kalman Filter, 132

GoS Grade of Service, 33

LKF Linearized Kalman Filter, 131

PF Particle Filter, 132

QoS Quality of Service, 33

RMSE Root Mean Square Error, 146

RNC Radio Network Controller, 37

RRM Radio Resource Management, 34

SIR Signal-to-Interference Ratio, 28

WCDMA Wideband CDMA, 37 


\section{1}

\section{Introduction}

This chapter is meant to provide an overall picture of the rest of the thesis. After a brief discussion on the background and objectives of the work in the next section, the remaining chapters of the thesis are addressed one by one in Section 1.2. Main contributions and concrete outcomes produced as the work progressed are summarized in Section 1.3.

\subsection{Background and Objectives}

Cellular radio systems providing speech service to users have been around long enough to be a part of the every day living for a many people. The mobile phone is one of the things you bring with you together with your wallet and keys as you walk on the street. Radio systems providing a multitude of services to the users, on the other hand, can not yet be called mature.

The radio resource management (RRM) problem can be defined as the problem of deciding what service to provide to each specific user. The increased complexity due to a multitude of services in the radio systems of today makes the RRM problem more challenging. In the systems considered in this work, using an inadequate RRM algorithm, the algorithm solving the RRM problem, does not only correspond to inefficient resource utilization, it can also mean that the ability to provide any service at all is jeopardized.

A fundamental criterion for a well operating RRM algorithm is accurate knowledge of the amount of available resources, both currently and after a possible RRM decision has been made. The systems considered in this work use code division multiple access (CDMA) as the scheme for sharing resources between users. Because of CDMA characteristics, the total amount of resources in the uplink, i.e., communication from mobile phone to the fixed base station, is not constant over time. Instead, it depends on where in the service area users are located. This makes it hard to decide how much of the resources that are currently used. Another word for this quantity, the ratio between used 
and total resources, is uplink relative load.

The objectives of this work have been to characterize, approximate and control the uplink load of a CDMA cellular system.

Characterizing Even though it is well known that the primary resource in the uplink is the total received power, it is interesting to derive a measure of how far the system is from being overloaded. The first objective of this thesis is to provide definitions of uplink load which can later be used in practice and/or theory.

Approximating A problem when trying to establish the uplink load in practice is that it is hard to measure the received power accurately. Furthermore, the uplink load measure used in practice also involves the unknown background noise power, which is why simply using measurements of the uplink received power is not recommended. The second objective of this thesis is to derive practically attractive approximations of the uplink load using only readily available information.

Controlling The ability to provide service in one area covered by cellular radio system depends on decisions made in other areas. Thus, making inappropriate decisions in the own geographical area can ruin the possibilities to provide services in the own area as well as in other areas. This indicates that centralized control should be applied. On the other hand, for increased performance, RRM decisions should be based on detailed information on the local radio environment and momentarily transmission requests. Therefore, both centralized and decentralized schemes seem to have advantages. The third objective of this thesis is to develop robust and efficient RRM algorithms based on the knowledge and experience gained from earlier parts of the thesis.

\subsection{Thesis Outline}

Below is a short explanation of the contents and purpose of the ten chapters in this thesis. Figure 1.1 provides an overview of where the chapters fit into an automatic control view of a cellular radio system.

Chapter 2 is a presentation of the results of the thesis. The chapter is, in terms of details, intermediate between the ordinary abstract and the entire thesis. Basic knowledge of cellular radio systems is here assumed to be known by the reader.

Chapter 3 provides fundamentals of systems for radio communications in general and CDMA cellular radio communications in particular along with the notation used throughout the thesis.

Chapter 4 introduces different aspects on uplink load in CDMA cellular radio systems. Uplink load from a practical point of view as well as from a more theoretical point of view is given. 


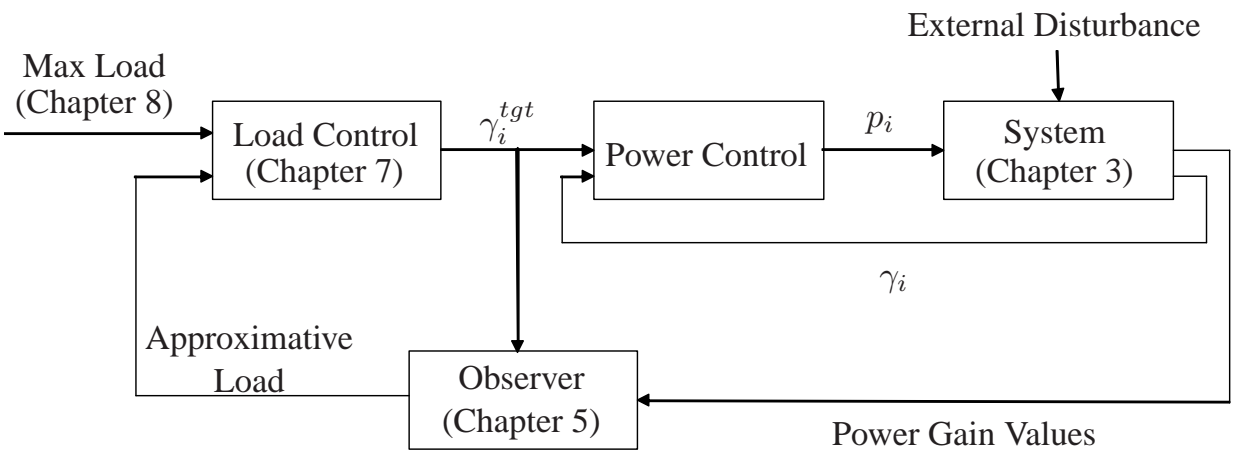

Figure 1.1: Resource control implemented as a cascade control system.

Once the uplink load is characterized, the remaining chapters contain the main results of this work. These results cover approximating and controlling the uplink load as well as using the developed framework for advanced filtering techniques.

Chapter 5 contains the derivation of a number of load approximations suited for practical use. Each approximation can be seen as an observer of the true system. The chapter also contains simulation where the approximations are evaluated under rather realistic circumstances.

Chapter 6 looks at relations between the load approximations derived in Chapter 5 and more theoretical aspects of uplink load. The analysis leads to a method for guaranteeing convergence of the uplink load approximations.

Chapter 7 uses experience from earlier chapters to design robust load controlling algorithms. Included in the chapter is a simulation study to give an idea of the performance of the algorithms.

Chapter 8 looks at the uplink load's role in the always present trade off between network performance and service quality for individual users. For a fixed maximum load, for example, higher bit rates can be given to fewer users, or coverage can be increased at the expense of less momentarily revenue for the operator.

Chapter 9 contains two applications of signal processing. The purpose of the first of these is to provide a more stable load approximation, while the second application uses nonlinear filtering to estimate the background noise power based on readily available measurements.

Chapter 10 contains the conclusions of the thesis. 


\subsection{Contributions and Publications}

The main contribution is the overall results on uplink load in CDMA cellular radio systems. The results cover characterizing, approximating and controlling the uplink load.

Detailed contributions are:

- Development and evaluation of centralized load approximations in Chapter 5.

- The framework including the system matrix introduced in Chapter 6. Much of the theoretical results are based on this framework.

- Development and evaluation of decentralized robust resource controlling schemes in Chapter 7.

Results presented in this thesis are partially covered by the following publications.

- The work on characterizing load in Chapter 4 is to a great extent also provided in

Erik Geijer Lundin and Fredrik Gunnarsson. Characterizing uplink load - concepts and algorithms. In Mohsen Guizani, editor, Wireless Communications Systems and Networks, chapter 14, pages 425-441. Kluwer Academic, 2003.

- The load approximations derived and evaluated in Chapter 5 were published in

Erik Geijer Lundin, Fredrik Gunnarsson, and Fredrik Gustafsson. Uplink load estimation in WCDMA. In Proceedings of the IEEE Wireless Communications and Networking Conference, New Orleans, LA, USA, March 2003c.

and

Erik Geijer Lundin, Fredrik Gunnarsson, and Fredrik Gustafsson. Uplink load estimates in WCDMA with different availability of measurements. In Proceedings of the IEEE Vehicular Technology Conference, Cheju, South Korea, April 2003a.

- Schemes for controlling uplink load are defined and evaluated in Chapter 7. This is based on

Erik Geijer Lundin, Fredrik Gunnarsson, and Fredrik Gustafsson. Robust uplink resource allocation in CDMA cellular radio systems. In Proceedings of the IEEE Conference on Decision and Control, Seville, Spain, December 2005a. To appear.

The work in Chapter 7 is based on the patent

Erik Geijer Lundin and Fredrik Gunnarsson. Using uplink relative path gain related measurements to support uplink resource management. US Patent Application No: 11/066,558. 
- A part of Chapter 8 treats performance trade offs in the presence of limited transmission powers. This was first published in

Erik Geijer Lundin, Fredrik Gunnarsson, and Fredrik Gustafsson. Uplink load and link budget with stochastic noise rise levels in CDMA cellular systems. In RVK05, Linköping, Sweden, June 2005 b.

- The adaptive filtering approach to load measure estimation in Chapter 9 was first published in

Erik Geijer Lundin, Fredrik Gunnarsson, and Fredrik Gustafsson. Adaptive filtering applied to an uplink load estimate in WCDMA. In Proceedings of the IEEE Vehicular Technology Conference, Cheju, South Korea, April 2003b.

- A part of the work is summarized in

Erik Geijer Lundin and Fredrik Gunnarsson. Uplink load in CDMA cellular radio systems. IEEE Transactions on Vehicular Technology, 2005. To Appear.

- Publications containing related work not included in this thesis are

David Törnqvist, Erik Geijer Lundin, Fredrik Gunnarsson, and Fredrik Gustafsson. Transmission timing - a control approach to distributed uplink scheduling in WCDMA. In Proceedings of the American Control Conference, Boston, MA, USA, June 2004.

Fredrik Gunnarsson, Erik Geijer Lundin, Gunnar Bark, and Niclas Wiberg. Uplink admission control in WCDMA based on relative load estimates. In IEEE International Conference on Communications, New York, NY, USA, April 2002. 


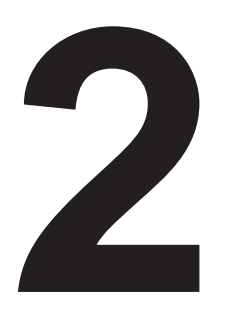

\section{Extended Summary}

The purpose of this chapter is to give a short version of the thesis, but yet elaborate on ideas and results. No complete results are given here, only enough to give an idea of what the corresponding parts of the thesis contain. As the sections in this chapter have the same name as the remaining chapters, it is hopefully fairly easy to find more detailed derivations and results.

Section 2.1 introduces the notation used. Definitions of uplink load are made and motivated in Section 2.2. Section 2.3 contains a short summary of the extensive work that has been on deriving and evaluating a number of load approximations. The framework for studying load from a more theoretical perspective and fundamental results are given in Section 2.4. Experience from Section 2.4 is then used in Section 2.5 to derive a number of robust algorithms for control of the uplink load. It is generally known that, for example, high load implies a tighter trade off between coverage, capacity and service quality for individual users. Both a theoretical and a more practical aspect of this trade off is touched upon in Section 2.6. Finally, signal processing is used in Section 2.7 to extract a more stable load approximation as well as estimating the background noise power.

\subsection{Cellular Radio Communication}

This section will only contain the introduced notation and is, unlike the corresponding chapter, not an attempt to introduce the reader to cellular radio communications in general. However, after having introduced the notation, the scope of the thesis is given from a automatic control point of view.

\subsubsection{System Model}

Consider the uplink, i.e., communication from user to base station, of a code division multiple access (CDMA) cellular radio system consisting of $M$ users and $B$ base stations, 
or cells. The radio channel between user $i$ and base station $j$ will simply be modeled as a power gain, $g_{i, j}<1$. Using this model, the received carrier power from user $i$ in base station $j$ is

$$
C_{i, j}=g_{i, j} p_{i}
$$

where $p_{i}$ is the power user $i$ transmits with.

The limiting resource in the uplink of a CDMA cellular radio system is often the total received interference power, $I^{\text {tot }}$. The total received interference power in base station $j$ is modeled as the background noise power, $N_{j}$, plus the sum of received carrier powers from users on the same frequency band,

$$
I_{j}^{t o t}=N_{j}+\sum_{i=1}^{M} C_{i, j}, \quad j=1,2, \ldots, B .
$$

For the purpose of maintaining a suitable received signal quality, power control is implemented in the systems considered here. It is useful to introduce the following notation for the base stations a user is power controlled by.

Definition 2.1 (Link Matrix). The element on row $i$ and column $j$ of the link matrix, $K \in \mathbb{R}^{M \times B}$, is defined as

$$
K_{i, j} \triangleq\left\{\begin{array}{l}
1, \text { if user } i \text { is power controlled by base station } j \\
0, \text { otherwise. }
\end{array}\right.
$$

A similar quantity is the set $K_{i}$ which contains the base stations that user $i$ is power controlled by, essentially

$$
K_{i} \triangleq\left\{j \mid K_{i, j}=1\right\} .
$$

The dual set is the set of users connected to a base station. For base station $j$,

$$
c_{j} \triangleq\left\{i \mid K_{i, j}=1\right\}
$$

The sets $K_{i}$ and power gain values are visualized in Figure 2.1. A user who is power controlled by several base stations, is said to be in soft handover between these cells. To characterize the number of cells a user may be power controlled by, the term connectivity is introduced.

Definition 2.2 (Connectivity). A system is said to have connectivity $k$ if at least one user is power controlled by $k$ base stations.

In a system with connectivity one, each set $K_{i}, i=1,2, \ldots, M$ contains only one base station. Uplink load is often related to uplink noise rise in the literature. The uplink noise rise is defined as

$$
\Lambda_{j} \triangleq \frac{I_{j}^{t o t}}{N_{j}} .
$$

The quality of the signal transmitted by user $i$ and received in cell $j$ is characterized by the carrier-to-interference ratio (CIR),

$$
\gamma_{i, j} \triangleq \frac{C_{i, j}}{I_{j}^{t o t}-C_{i, j}}
$$




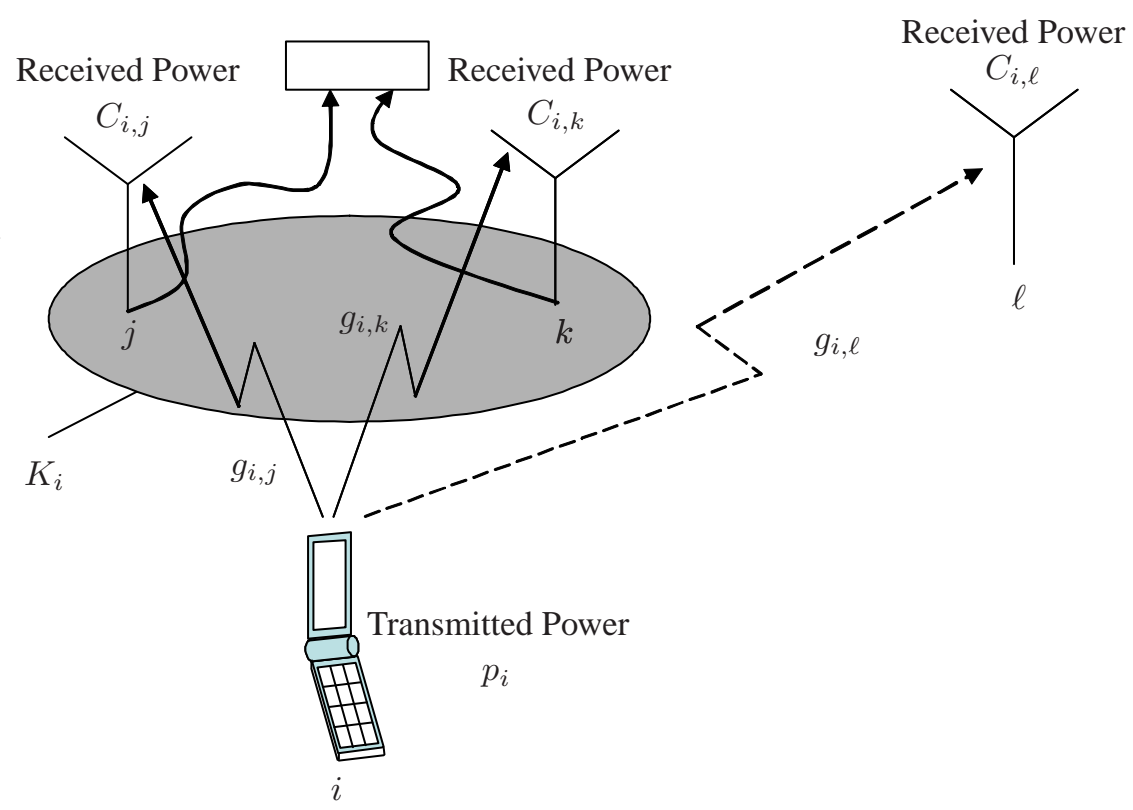

Figure 2.1: Variables in a cellular radio system.

For notational ease, carrier-to-total-interference ratio (CTIR), $\beta$, is introduced,

$$
\beta_{i, j} \triangleq \frac{C_{i, j}}{I_{j}^{t o t}} .
$$

The relations between $\gamma$ and $\beta$ are simply

$$
\beta=\frac{\gamma}{1+\gamma} \text { and } \gamma=\frac{\beta}{1-\beta}
$$

The total perceived signal quality is related to the CIR and CTIR obtained by combining the signals received in different base stations. These total CIR and CTIR will simply be denoted by $\gamma_{i}$ and $\beta_{i}$, respectively. For example, in case of connectivity one,

$$
\gamma_{i}=\gamma_{i, K_{i}} \text { and } \beta_{i}=\beta_{i, K_{i}}
$$

\subsubsection{Assumptions and Scope of the Thesis}

Communication systems are among the most complex systems built by man. As such, there is much to gain from breaking down the system into smaller subsystems, which can be handled relatively independently. Besides splitting the system into uplink and downlink, mechanisms of the system operating on different time scales can be separated.

Systems operating on different time scales are often stumbled upon within automatic control. The different time scales make the system well suited for a cascade control 
framework, as in Figure 1.1. In the inner loop, where fast updates are made, power control adjusts the users' transmission powers on a time scale of milliseconds. The purpose of the power control loop is to adjust the transmission powers to maintain an experienced CIR approximately equal to a target CIR, despite variations in the radio environment and background noise. The power control can only succeed if the individual target CIR values, $\gamma_{i}^{t g t}$ for each user $i$, are not set too high. The adjustment of the target CIR values is done by a number of load controlling algorithms in the outer loop operating at a far slower rate than the power control. It is customary to analyze the inner and outer loop of a cascade system independently, which is motivated by the considerable difference in time scale. When analyzing the outer loop, the inner loop is often assumed to operate perfectly on the time scale that the outer loop operates on. This work is entirely devoted to the load control part. Motivated by the cascade control view, it is always assumed that the system has perfect power control when deriving the results.

\subsection{Characterizing Uplink Load}

Example 2.1 is meant to give an intuitive view of one of the two definitions of uplink load that will be made, the noise rise relative load.

\section{Example 2.1: Interference Limited System}

Consider a system consisting of just one base station, i.e., $B=1$. The total received interference power is

$$
\begin{array}{r}
I_{1}^{t o t}=N_{1}+\sum_{i=1}^{M} C_{i, 1}=N_{1}+\sum_{i=1}^{M} \beta_{i} I_{1}^{t o t} \Leftrightarrow \\
\Lambda_{1}=\frac{I_{1}^{t o t}}{N_{1}}=\frac{1}{1-\sum_{i=1}^{M} \beta_{i}} .
\end{array}
$$

The nature of $I_{1}^{\text {tot }}$ implies that it should be positive. Thus, a basic requirement is

$$
\sum_{i=1}^{M} \beta_{i}<1 .
$$

This puts a constraint on the maximum combined CTIR that the users can have, regardless of how much transmission power they have available.

Because of the polynomial in the denominator of (2.2), this is often called the pole equation (Holma and Toskala, 2000). The above example shows that the system's ability to provide service to the users is limited, despite access to infinite transmission powers. This is true also for a system consisting of several cells. A system with this property is called an interference limited system.

Noise Rise Relative Load. Since the system is interference limited, a definition of uplink load should be related to the received interference power. The most common 
definition of uplink load found in the literature is

$$
L^{n r} \triangleq 1-\frac{N}{I}=1-\frac{1}{\Lambda} .
$$

The index $n r$, which is an acronym for noise rise, has been added to separate this load from the other type of load defined below. This type of load, which will be called noise rise relative load is especially used for practical applications in the literature. The definition is natural, considering that the uplink interference power is the primary uplink resource. Note that a noise rise relative load of zero corresponds to a noise rise of one, i.e., $I^{t o t}=N$.

When introducing this type of load, it is common to talk about the pole capacity. This is the capacity, in whatever measure used, of the system when the noise rise relative load reaches one. This is a theoretical capacity since a noise rise relative load of one corresponds to infinite interference powers.

For the purpose of establishing the noise rise relative load in practice, the total received interference power is often split into three parts. One being the background noise and the other two are interference from users connected to the own base station, intracell interference, and interference from users connected to other base stations, intercell interference,

$$
I^{\text {tot }}=N+I^{\text {own }}+I^{\text {other }} .
$$

The intercell interference depends on where users are located in the system. This means that also the system's pole capacity depends on where users are located and how many users there are in different cells. The soft capacity of a system can only be reached if soft characteristics, such as received interference power or power gain values between users and base stations, are studied in the radio resource management (RRM). So, for example, an RRM algorithm studying the system's hard capacity, as in for example counting the number of users or measuring the throughput of the system, can not fully utilize the system's resources.

A common way of approximating the uplink load is to use an intercell-to-intracell factor, $f$. When doing so, the intercell interference is assumed to be a fraction $f$ of the intracell interference, $I^{\text {other }}=f I^{\text {own }}$. The uplink load in base station $j$ is then approximated by

$$
L_{j}^{n r} \approx \frac{1}{1-(1+f) \sum_{i \in c_{j}} \beta_{i}^{t g t}} .
$$

The soft capacity of the system can not be achieved when using this method.

There are several reasons for operating a system at lower load than one. Lower load means lower noise rise. A high noise rise indicates a high total interference power, something which can cause problems with coverage as the users have limited transmission powers. Another reason is that it is harder for the power control to operate satisfactory at a high noise rise level. Loosely speaking, small changes in the radio environment or small changes by the load control algorithm in Figure 1.1 will make much higher impact at high noise rise levels, see Figure 2.2.

Feasibility Relative Load. The second type of studied uplink load is related to the existence of finite transmission powers to support the service requested by the load control. A system is feasible if it exists finite transmission powers to support the requested 


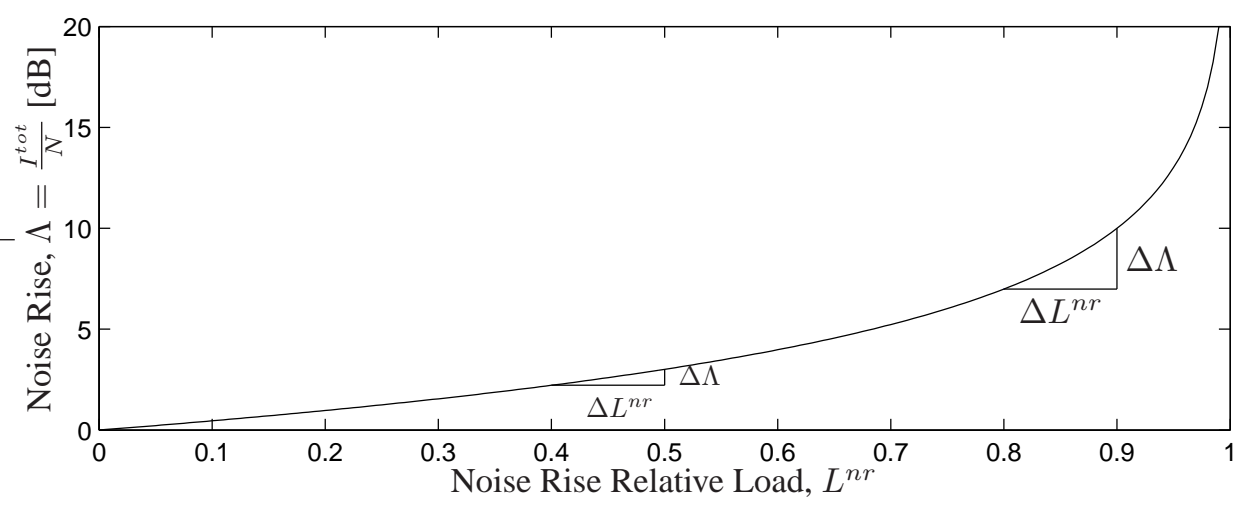

Figure 2.2: The nonlinear relation between noise rise relative load, $L^{n r}$, and noise rise, $\Lambda$. Additional load, $\Delta L^{n r}$, gives different noise rise contribution, $\Delta \Lambda$, at different load levels.

signal qualities (Zander, 1993). If that is not the case, the system is infeasible. The uplink feasibility relative load is defined as one over the factor by which all users' target CTIR can be scaled by while maintaining a feasible system,

$$
L_{f} \triangleq \sup _{\mu}\left\{\frac{1}{\mu}: \mu \beta_{i}^{t g t} \text { leads to a feasible system }\right\} .
$$

From the definition, it is clear that a feasibility relative load less than one, $L_{f}<1$, corresponds to a feasible system.

The noise rise relative load is related to the load in a specific base station while the feasibility relative load is related to the entire system. Furthermore, the feasibility relative load is more of a theoretical load, since it only applies as long as the users have transmission powers enough to support their target CTIR values. The noise rise relative load, on the other hand, is always applicable and much easier to establish in a general, practical system. A more detailed comparison between the two load definitions is made in Chapter 6. It is concluded, for example, that as long as all users can maintain their target CIR in a system with connectivity one, the feasibility relative load is lower than or equal to the noise rise relative load, i.e., $L_{f} \leq L^{n r}$. Equality holds only in a single cell system.

\subsection{Approximating Uplink Load}

A major part of this work has been spent on deriving and evaluating the performance of a number of uplink load approximations of the uplink noise rise relative load. Using only readily available information on the users' target CTIR values and the power gain between users and base stations, they provide approximations that are for free in a sense since they do not require any additional signaling.

Due to the intercell interference part of the total inteference power, the noise rise relative load in one base station depends on the situation in the surrounding cells. This 
yields that an approximation in a base station using only local information can not consider the whole interference power explicitly. The proposed load approximations, on the other hand, use information gathered in several base stations and can therefore explicitly consider all contributions to the total received interference power.

\subsubsection{Derivation of Load Approximations}

Inspired by what is done in Wideband CDMA (WCDMA), a system utilizing soft handover is studied in Chapter 5. A consequence of utilizing soft handover is that the sets $K_{i}$ may contain several base stations. This makes the relation between the interference power in different base stations quite complex. To exemplify, first approximate the combination of signals received from one user in different cells with maximum ratio combining. Using maximum ratio combining implies that the CIR of the combined signal equals the sum of the separately received signals' CIR, i.e.,

$$
\gamma_{i}=\sum_{k \in K_{i}} \gamma_{i, k}
$$

By neglecting the power control errors, received CIR can be approximated with target CIR. Finally, transforming CIR into CTIR and using the model of total received interference power in (2.1) at time $t$ yields

$$
I_{j}^{t o t}(t)=N_{j}(t)+\sum_{i=1}^{M} p_{i}(t) g_{i, j}(t) \approx N_{j}(t)+\sum_{i=1}^{M} g_{i, j}(t) \frac{\beta_{i}^{t g t}(t)}{\sum_{k \in K_{i}} \frac{g_{i, k}(t)}{I_{k}^{t o t}(t)}}, j=1,2, \ldots, B .
$$

An important property of the above equation is that it only contains variables that can be expected to be known in a central node, i.e., the users' target CTIR and measured power gain values, and the quantity that will be solved for, $I_{j}^{t o t}(t), j=1,2, \ldots, B$.

Considering that there is one equation like (2.4) in each base station $j$, calculating either the uplink noise rise, $I_{j}^{t o t} / N_{j}$, or the uplink noise rise relative load involves solving a system of nonlinear equations. Two methods for doing this are proposed in Chapter 5, one is based on linearization and the other uses fix point iterations.

Linearization. By approximating $I_{k}^{t o t}(t)$ with $I_{j}^{t o t}(t)$ in $(2.4)$, the uplink noise rise relative load in base station $j$ at time $t$ can be approximated by

$$
L_{j}^{l i n}(t)=\sum_{i=1}^{M} \beta_{i}^{t g t}(t) \frac{g_{i, j}(t)}{\sum_{k \in K_{i}} g_{i, k}(t)} .
$$

The index lin has been added to emphasize the linearity in the users' target CTIR. This expression should be compared to (2.3), which relates to a single cell system. Since all users in the system is considered by $L_{j}^{\text {lin }}$, not only the load caused by users connected to the own cell, but also the load caused by users connected to other base stations are explicitly considered. There is thus no need for an intercell-to-intracell factor as is usually the case in the literature. 
Fix Point Iterations. The system of nonlinear equations defined by (2.4) can be solved through fix point iterations. by approximating the background noise power in all base stations with a common, but yet unknown, background noise power, i.e., $N_{j}(t) \approx N(t)$ for all $j$, the nonlinear equations can be approximately solved using fix point iterations, as in Algorithm 2.1. The parameter $N^{i t e r}$ is the number of fix point iterations performed before each update. The analysis in Chapter 6 yields that Algorithm 2.1 converges to the

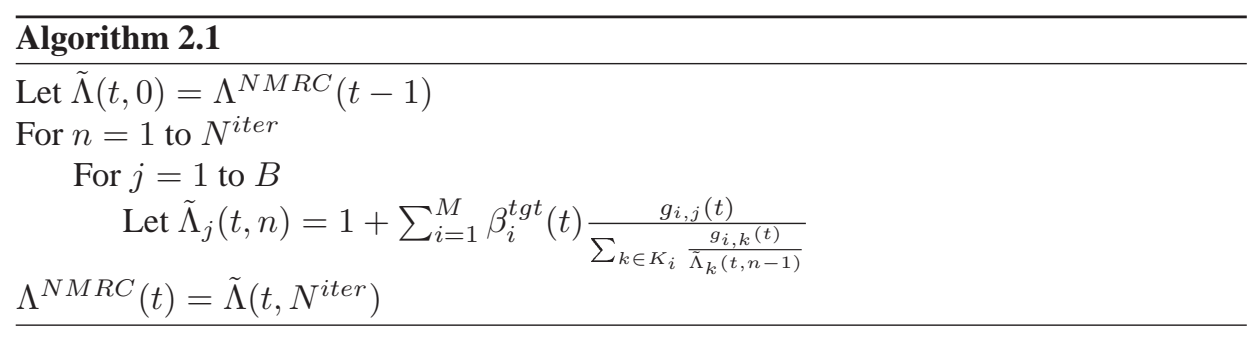

true noise rise if provided with accurate power gain values and applied in a system with connectivity one.

As the approximations derived here, $L_{j}^{l i n}$ and $\Lambda_{j}^{N M R C}$, study the users' relative power gain and all cells simultaneously, a resource management algorithm using these approximations can in fact achieve the system's soft capacity.

\subsubsection{Error Sources}

Besides the approximations made during the derivation, examples of the error sources that appear in practice are the following.

- Inaccurate and incomplete knowledge of the power gain between users and base stations.

- Inaccurate assumption on how signals received in different cells are combined.

- TX increase, which is the increase in average intercell interference power due to fast power control.

\subsubsection{Evaluation}

A rather complex simulator has been used to perform an extensive simulation study on the performance of the uplink load approximations. Many weaknesses of a true system is modeled, such as imperfect power control and sparsely sampled power gain reports that do not include all base stations. The results from this study are reported in Chapter 5. The simulator models many characteristics of a true system such as fast fading, decoding ability, soft handover and user mobility.

When deriving (2.4), which defines the system of nonlinear equations that is the bases for the approximations, maximum ratio combining of the signals was assumed. Similar expressions as (2.4) are derived by assuming selection combining ${ }^{1}$ or the actual mix of

\footnotetext{
${ }^{1}$ When combining signals using selection combining, the CIR of the combined signal is the maximum CIR of the separately received signals.
} 
maximum ratio and selection combining that is used in the system. Applying fix point iterations to these systems of nonlinear equations leads to two more approximations of the uplink noise rise. These will be referred to as $\Lambda^{N S E L}$ and $\Lambda^{N B O T H}$, respectively. Figure 2.3 shows the average error of the approximations with and without a compensation for the TX increase contribution to the true noise rise. The compensation requires knowledge of the characteristics of the specific channels. The simulations indicate that

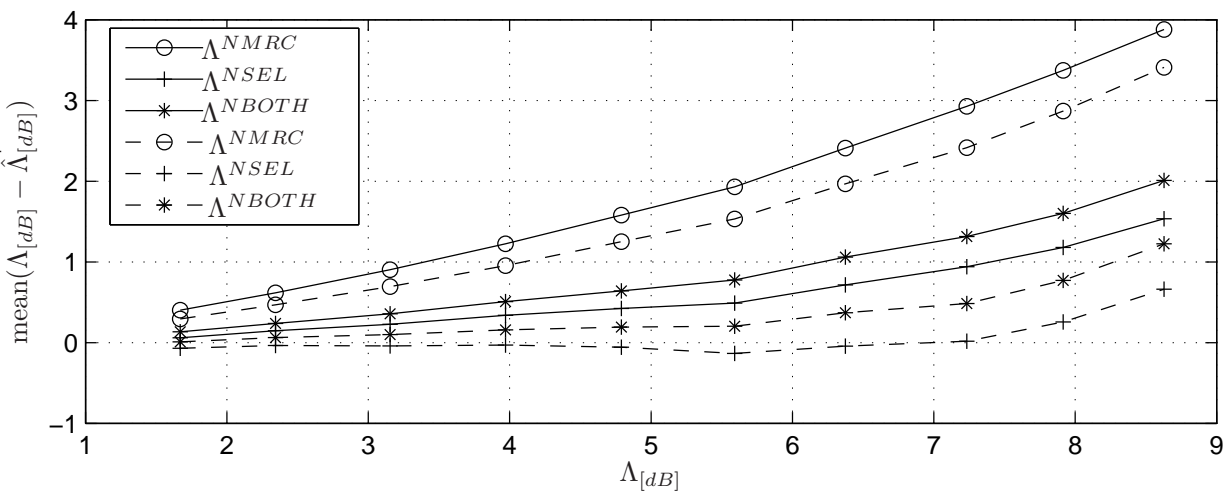

Figure 2.3: Error in noise rise with 64 kbps users. The dashed lines represents approximations with the proposed compensation for TX increase.

it is possible to approximate the uplink load to within $1 \mathrm{~dB}$ for as high noise rise levels as 8 or even $9 \mathrm{~dB}$. Even though not shown here, the variance of individual approximation errors is fairly small. The TX increase compensation comes with a slight increase in variation in the errors.

Similar results for the approximations using linearization in Section 2.3.1 are reported on in Chapter 5.

\subsection{Analyzing Uplink Load}

Relations between noise rise relative load and feasibility relative load are derived in Chapter 6. These relations, together with relations to the uplink load approximations are also found in Chapter 6, are used to provide a criteria for system feasibility. The results are divided into those applicable to a system with connectivity one, and those applicable to systems with higher connectivity.

A basis for much of the work in Chapter 6 is a framework using a matrix expression for the total received interference powers in the base stations. In order to derive the matrix, consider a system with connectivity one. This means that (2.4) simplifies to

$$
I_{j}^{t o t}(t)=N_{j}(t)+\sum_{i=1}^{M} \beta_{i}^{t g t}(t) \frac{g_{i, j}(t)}{g_{i, K_{i}}(t)} I_{K_{i}}^{t o t}(t)=N_{j}(t)+\sum_{i=1}^{M} \beta_{i}^{t g t}(t) z_{i, j}(t) I_{K_{i}}^{t o t}(t) .
$$


Here, $z_{i, j}(t)$ is the relative power gain which is defined for any connectivity as

$$
z_{i, j}(t) \triangleq \frac{g_{i, j}(t)}{\sum_{k \in K_{i}} g_{i, k}(t)} .
$$

The duality between $K_{i}$ and $c_{k}$ implies $K_{i}=k, \forall i \in c_{k}$. The total interference power in a system with connectivity one can therefore be expressed as

$$
I_{j}^{t o t}(t)=N_{j}(t)+\sum_{k=1}^{B} \sum_{i \in c_{k}} \beta_{i}^{t g t}(t) z_{i, j}(t) I_{K_{i}}^{t o t}(t)=N_{j}(t)+\sum_{k=1}^{B} L_{k, j}(t) I_{k}^{t o t}(t),
$$

where

$$
L_{k . j}(t) \triangleq \sum_{i \in c_{k}} \beta_{i}^{t g t}(t) z_{i, j}(t), k, j=1,2, \ldots, B .
$$

Each element $L_{k, j}(t)$ can be interpreted as the load that users power controlled by base station $k$ causes in base station $j$ at time $t$. Compiling all $L_{k, j}$ into a matrix $L=\left[L_{k, j}\right]$ yields the system matrix. A matrix expression for the total received interference in all base stations in a system with connectivity one is thus

$$
I^{t o t}=N+L^{T} I^{t o t} .
$$

This expression is used repeatedly in Chapter 6 to derive various results. For example, the feasibility relative load of a system with connectivity one is the maximum eigenvalue of the system matrix, i.e.,

$$
L_{f}=\bar{\lambda}(L),
$$

where $\bar{\lambda}(L)$ is the eigenvalue of the system matrix $L$ with maximum magnitude.

Other results found in Chapter 6 give several bounds on the uplink feasibility relative load, bounds that are possible to calculate before a resource management decision is made. Feasibility relative load is also related to convergence of the fix point iterations described in Section 2.3.1. For example, it is shown that Algorithm 2.1 converges to the true noise rise vector in a feasible system with connectivity one. The results found in Chapter 6 are summarized in a procedure for approximating uplink load of a system with arbitrary connectivity.

\subsection{Controlling Uplink Load}

Various properties of the system matrix, whose elements are given by (2.5), are combined with experience from the theoretical analysis to design resource allocation algorithms in Chapter 7. More concrete, the proposed allocation algorithms are optimization problems in which the constraints are inspired by the relations given by the theoretical analysis. The utilization function in these problems is the sum of maximum achievable rate normalized with the signal bandwidth (Wozencraft and Jacobs, 1965, page 520),

$$
\sum_{i} \log _{2}\left(1+\gamma_{i}^{t g t}\right)
$$

Essentially, two resource allocation algorithms are proposed. They both make resource allocations in local nodes while maintaining system feasibility. Neither of the algorithms rely on measurements of the uplink noise rise. 
Decentralized Algorithm. The first proposed algorithm does not use a central node at all. The algorithm is based on a result applicable to all square matrices,

$$
\bar{\lambda}(L) \leq\|L\|_{\infty}
$$

Since all elements of $L$ are positive, the matrix infinity norm is simply the maximum row sum.

A single base station can control all elements of a row in the system matrix $L$. Therefore, if all base stations make resource assignments such that the corresponding row is less than some constant $L_{f}^{t g t}$, so will the feasibility relative load, $L_{f}$, be. Obviously, by choosing $L_{f}^{t g t}$ less than one, this yields a method for guaranteeing system feasibility.

A result by Gantmacher (1974) states that a lower bound on $\bar{\lambda}(L)$ is given by the minimum row sum. Therefore, if all row sums equal $L_{f}^{t g t}$, so does $L_{f}$.

The optimization problem solved in each base station $k$ at each time instant $t$ is

$$
\begin{aligned}
& \max _{\beta_{i}^{t g t}(t) \in c_{k}} \sum_{i \in c_{k}} \log _{2}\left(1+\gamma_{i}(t)\right)=-\sum_{i \in c_{k}} \log _{2}\left(1-\beta_{i}^{t g t}(t)\right) \\
& \text { s.t. }\left\{\begin{array}{l}
\sum_{i \in c_{k}} \beta_{i}^{t g t}(t) \sum_{j=1}^{B} z_{i, j}(t) \leq L_{f}^{t g t} \\
\beta_{\text {min }} \leq \beta_{i}^{t g t}(t) \leq \beta_{\text {max }}, \forall i \in c_{k} .
\end{array}\right.
\end{aligned}
$$

Semi-Centralized Algorithm. The second proposed algorithm uses a central node to distribute resource pools to the base stations. Each base station then assigns resources to the users connected to it. System feasibility is guaranteed through the use of a mutual agreement between the central node and the base stations.

The purpose of the central node is to distribute resource pools to the base stations. Typically, a base station with many users in it should receive a larger resource pool. By feeding back information on where in the radio environment the users are located, soft capacity can be studied in the central node just as it is in the base stations. Feeding complete information on each users' location would require too much signaling. The information send back from base station $k$ at time $t$ is

$Y_{k, j}(t) \triangleq \frac{1}{\sum_{i \in c_{k}} \beta_{i}^{t g t}(t-1)} \sum_{i \in c_{k}} z_{i, j}(t-1) \beta_{i}^{t g t}(t-1)=L_{k, j}(t-1), j=1,2, \ldots, B$

Based on the information received from all the base stations, the central node compiles a matrix $\bar{L}=\left[\bar{L}_{k, j}\right]$, with

$$
\bar{L}_{k, j}=s_{k} Y_{k, j}(t)
$$

The resource pools, $s_{j}(t), j=1,2, \ldots, B$, are given in the central node as the solution to 
the following optimization problem

$$
\begin{aligned}
\max _{s} & \sum_{j=1}^{B} s_{j}(t) \\
\text { s.t. } & \left\{\begin{array}{l}
\left(\begin{array}{cc}
E & \bar{L} \\
\bar{L}^{T} & L_{f}^{t g t^{2}} E
\end{array}\right) \succeq 0 \\
\bar{L}_{k, j}=s_{k}(t) Y_{k, j}(t), k, j=1,2, \ldots, B \\
s_{k}(t)=\sum_{i \in c_{k}} \beta_{i}^{t g t}(t) \\
\beta_{\text {min }} \leq \beta_{i}^{t g t}(t) \leq \beta_{\max }, i=1,2, \ldots, M .
\end{array}\right.
\end{aligned}
$$

Here, $E$ is the identity matrix. The matrix inequality will guarantee that the maximum eigenvalue of $\bar{L}$ is less than or equal to the target feasibility relative load, $L_{f}^{t g t}$. Now, if each element $L_{k, j}(t)$ in the system matrix is less than or equal to the corresponding element in $\bar{L}$, the maximum eigenvalue of $L(t)$ will also be less than $L_{f}^{t g t}$. This requirement will be guaranteed by the way resources are assigned in the local nodes (the base stations). Upon receiving the resource pool $s_{k}(t)$, the resource assignment made by local node $k$ will be the solution to the following optimization problem

$$
\begin{aligned}
& \max _{\beta_{i}^{t g t}(t) \in c_{k}} \sum_{i \in c_{k}} \log _{2}\left(1+\gamma_{i}(t)\right)=-\sum_{i \in c_{k}} \log _{2}\left(1-\beta_{i}^{t g t}(t)\right) \\
& \text { s.t. }\left\{\begin{array}{l}
\sum_{i \in c_{k}} \beta_{i}^{t g t}(t) z_{i, j}(t) \leq Y_{k, j}(t) s_{k}(t) \forall j \\
\beta_{\text {min }} \leq \beta_{i}^{t g t}(t) \leq \beta_{\max }, \forall i \in c_{k} .
\end{array}\right.
\end{aligned}
$$

After the base stations have assigned target CTIR values, they calculate new values $Y_{k, j}(t+1)$ and send them back to the central node. Using this iterative procedure, repeatedly solving the optimization problems in the different nodes, implies that the algorithm can adapt to changes in the radio environment as well as to users moving between cells.

Simulations. For comparison, two additional algorithms are introduced. One is a centralized algorithm with complete knowledge of the radio environment in the entire system. This algorithm is meant to give an idea of what can be achieved. The second algorithm introduced for comparison do not use a central node nor relative power gain values, and will therefore be referred to as the blind algorithm. Using this algorithm, each base station has a resource pool of $s_{0}$ which it shares evenly over users connected to it. Figure 2.4 shows the result of a simulation study in which a small part of the service area has a considerably higher user density. This implies that there is performance to gain by moving resources between base stations.

According to Figure 2.4a, both of the proposed robust algorithms provide practically equal capacity as the completely centralized, while the blind algorithm gives significantly lower capacity. Figure $2.4 \mathrm{~b}$ shows the relative success rate of the robust algorithms. The semi-centralized algorithm's ability to distribute resources between base stations results in a higher success rate for low target load levels. For example, in this specific scenario, choosing the target feasibility relative load to 0.5 yields that the semi-centralized algorithm succeeds in approximately $80 \%$ of the cases while the decentralized algorithm only 
a)

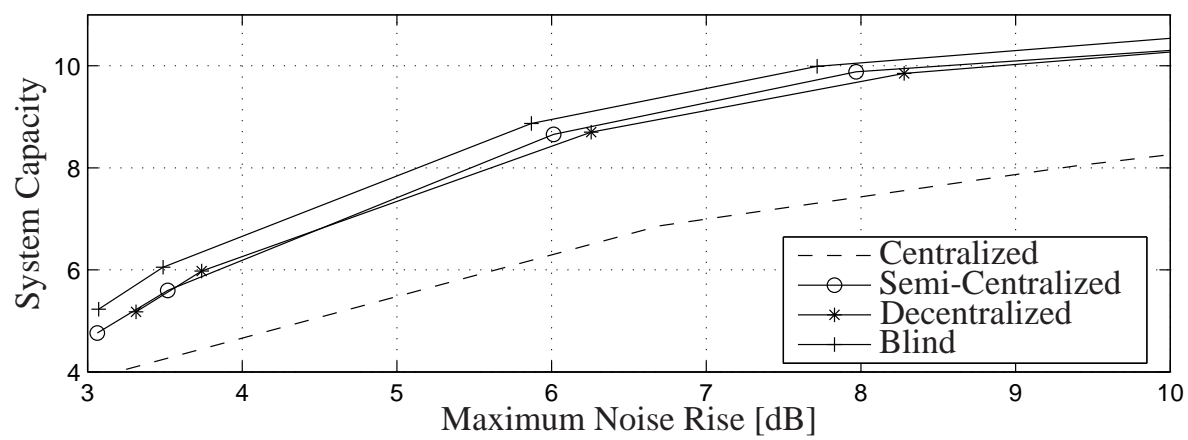

b)

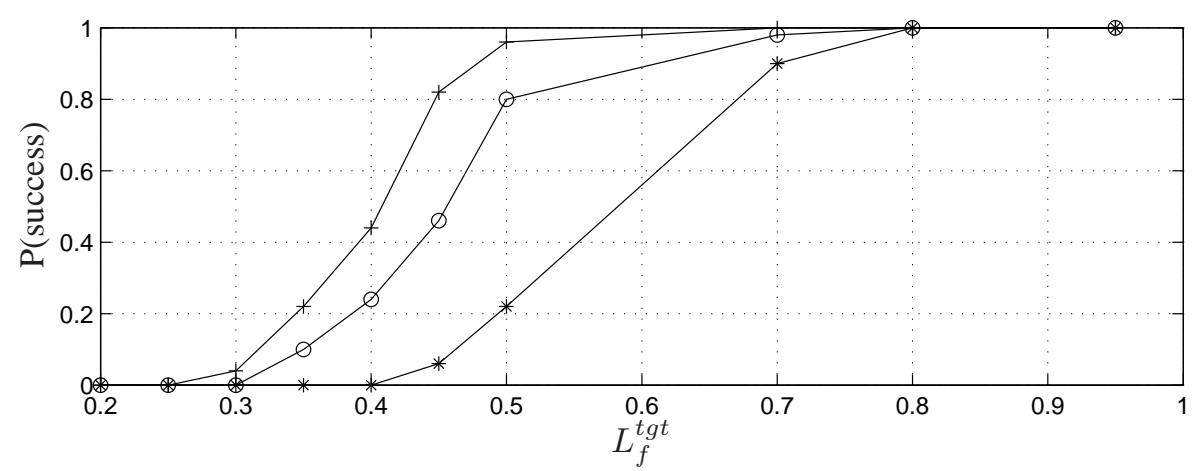

Figure 2.4: Comparison of radio resource management algorithms. $\beta_{\min }=-15 \mathrm{~dB}$ $\left(\gamma_{\min } \approx-15 \mathrm{~dB}\right), \beta_{\max }=0.5\left(\gamma_{\max }=1\right)$. 50 Monte Carlo simulations.

manages to find a solution in $20 \%$ of the cases. Considering higher offered load, these success rates would appear for a higher target feasibility relative load. The difference in success rates between the different algorithms is due to the semi-centralized algorithm's ability to distribute resources between the local nodes.

As a conclusion, by studying the relative power gain it is possible to design decentralized algorithms that provide a throughput comparable to that given by a completely centralized algorithm. This is done with considerably less, or no, signaling between local and central nodes, without neglecting the robustness in terms of guaranteed feasibility. Furthermore, since the algorithms use local nodes, they can take advantage of local information on for example the radio environment.

\subsection{Feasibility versus Coverage}

Thus far, focus has been on approximating or controlling the uplink load. By using a few examples, the trade off between capacity, coverage and quality of service for individual users is addressed in Chapter 8.

Assuming that the users have transmission powers to support their service, the feasi- 
bility relative load in a single service scenario with two cells and one user in each is given by (dropping the time index, $t$ )

$$
L_{f}=\beta_{0}\left(1+\sqrt{z_{1,2} z_{2,1}}\right) \Leftrightarrow \sqrt{z_{1,2} z_{2,1}}+1=\frac{L_{f}}{\beta_{0}},
$$

where $\beta_{0}$ is the target CTIR values for the only service provided. The right hand side relation indicates a possible trade off between coverage, in terms of users' relative power gain, and capacity in terms of the target CTIR. This shows that the coverage is limited in a multi cell scenario, even if the users have unlimited transmission powers. As isolated cells correspond to zero relative power gain to other cells, better isolation between cells in a system yields better capacity or possible higher service quality for the users. A possible limit on the uplink noise rise is in this case arbitrary, since it is the feasibility requirement that provides the limitations. In a scenario where the relative power gain values are small, it is primarily the target CTIR, $\beta_{0}$, that is constrained. This is scenario is referred to as a capacity limited scenario.

Now consider limited transmission powers. In this case it is perhaps wise to choose a low noise rise target for the resource allocation algorithms, in order to not lose too much coverage. A stochastic approach to link budgets has been applied to calculate an approximative relation between target CTIR, coverage and grade of service in a few example scenarios. Figure 2.5 shows the relation between maximum allowed noise rise relative load and cell radius. It is clear that the noise rise relative load that a system can cope with in practice decreases fast as the cell radius grow, especially in system deployments with large cells. The actual numbers on the $\mathrm{x}$-axis depend on the specific scenario studied, such as the background noise power and maximum user transmission power. The shaded area is where the system can be expected to be capacity limited, as opposed to coverage limited.

A conclusion of this analysis, is thus that the target load for the resource allocation algorithms depends on the specific power gain distribution. In some scenarios, the target load is set by coverage demands, while in others it is the feasibility requirement that gives the maximum allowed load. The system may thus be coverage limited or capacity limited. Since the distribution depends on the specific system deployment such as the size of the cells and antenna characteristics, it can be changed to some extent by choosing a different system deployment.

\subsection{Filtering and Estimating Uplink Load}

Signal processing techniques have been used in two different applications in Chapter 9. These two are here explained in separate subsections.

\subsubsection{Noise Rise Relative Load Filtering}

The noise rise relative load is constantly oscillating about a load level, as can be noticed in practice. If these oscillations can be canceled, resource management algorithms can be more aggressive, leading to better resource allocation. An ordinary auto-regressive (AR) signal model, describing oscillations with zero mean, was extended to a biased $A R$ 


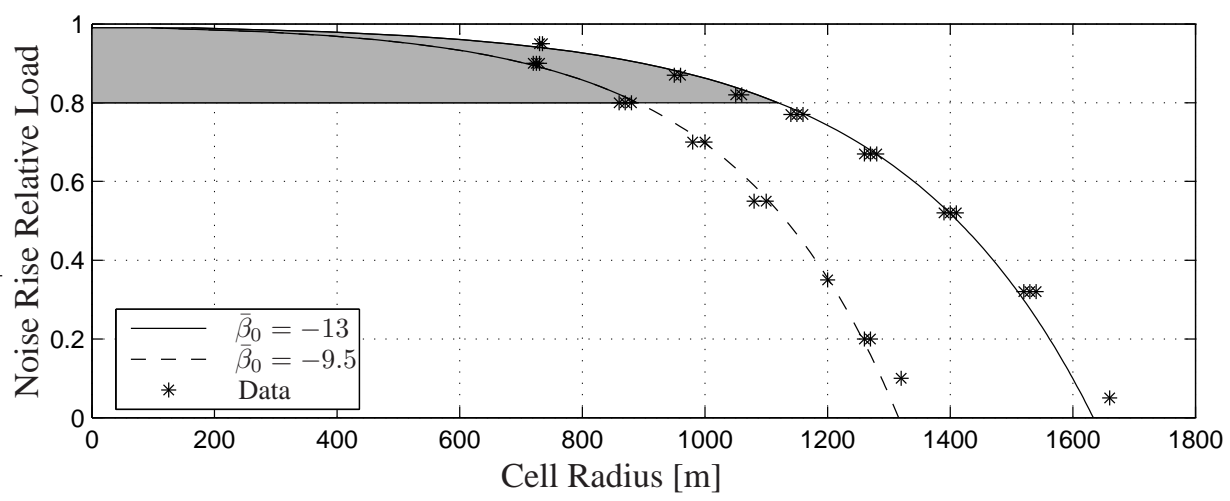

Figure 2.5: Relation between average noise rise relative load and cell radius when the users have a 95\% probability of experiencing coverage. Two different target CTIR values are considered. A simple model has also been fitted to data. The shaded area represents situations where the system is capacity limited, as opposed to coverage limited. $A \bar{\beta}_{0}$ of $-9.5 \mathrm{~dB}$ approximately corresponds to a $384 \mathrm{kbps}$ service and $\bar{\beta}_{0}=-13$ to a $144 \mathrm{kbps}$ service.

model, describing oscillations around an arbitrary level. In the application, this level is considered time varying and it is the primary quantity to estimate.

The developed signal model, Kalman filtering and change detection are applied to a signal produced by the load approximations derived in Section 2.3. The result is a more stable load approximation which is alert to sudden changes in the load level as well as an estimate of the load levels derivative. Ordinary low pass filtering of the signal would either be very slow to adapt to a new load level or not suppress the oscillations to the same extent as the Kalman filter. Figure 2.6 shows an example of how the estimation quickly adapts to a new load level, while simply low pass filtering the estimate results in slow adaptation to a new load level.

\subsubsection{Background Noise Power Estimation}

When using measurements of the uplink interference power for resource management, an inaccurate measure of the background noise power can lead to decreased performance in terms of capacity and coverage. Signal processing is used to estimate the background noise power using only available measurements of the uplink interference power. A nonlinear signal model based on the system matrix, describing the relation between background noise power and measured received interference power, is developed. The model incorporates uplink interference power measurements being corrupted by a base station individual bias in logarithmic scale.

As a nonlinear signal model is used, the estimation performance can be improved by using nonlinear filtering. Besides linearizing the state space model and applying a Kalman filter, extended Kalman filter (EKF) and particle filters have been applied.

The EKF, in general, uses a linearized version of the nonlinear model where the linearization point is repeatedly chosen to the latest estimated state variables. The particle 


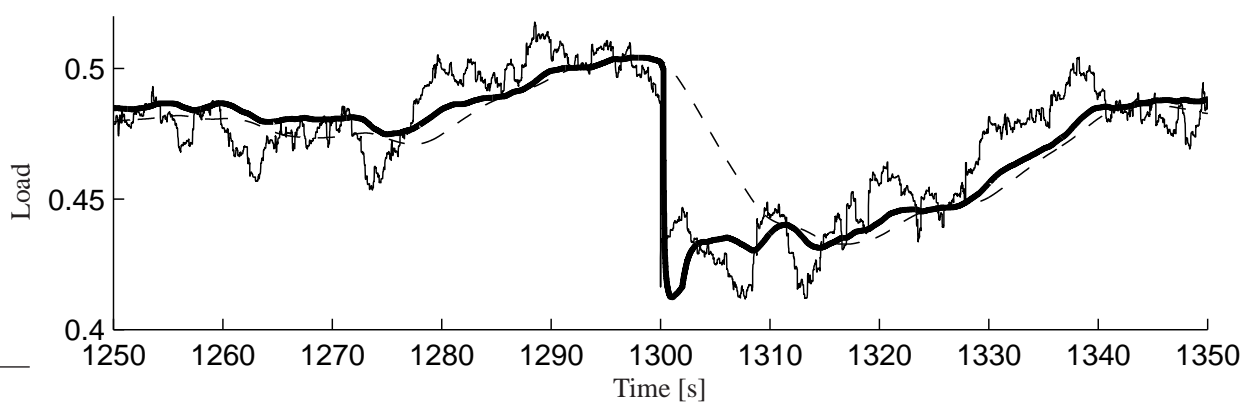

Figure 2.6: Example of estimated average load level when simply low pass filtering (dashed) and using a biased AR-model together with Kalman filtering and change detection (solid thick). The thin solid line is the load approximation used as input to the estimation process.

filter uses Monte Carlo integration to approximate the probability density function for the true state space vector. The main strength with particle filters is that almost arbitrary probability density functions for the measurement and process noise can be modeled. When using particle filters a nonlinear signal model does not have to be linearized at any stage.

The application performs well, despite the rather unrealistic circumstances assumed during the derivation. Even when soft handover is used in the simulations, unlike in the modeling, the algorithm manages to estimate the background noise power with usually less than $1 \mathrm{~dB}$ error in bursty traffic. Figure 2.7 shows how an EKF and two different particle filters with 5000 and 10000 particles, respectively adapts to a sudden change in the background noise power in one out of nine base stations. Using more particles implies a better approximation of the probability density function for the possible values of the state vector. However, in this case, 5000 particles seem to be enough to still detect the sudden change in the true background noise power. 


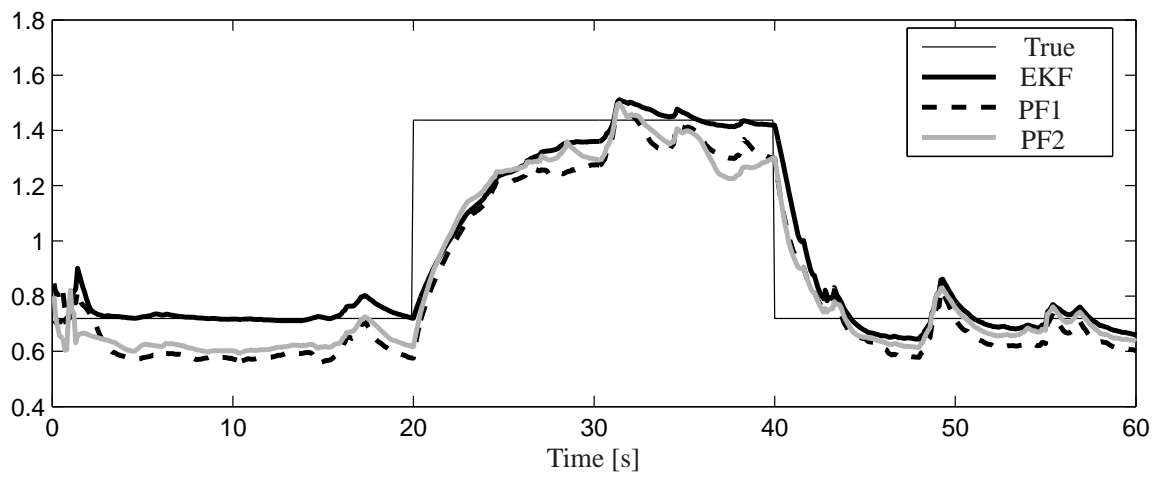

Figure 2.7: Example of background noise power estimation. The true background noise power makes jumps at $t=20 \mathrm{~s}$ and $t=40 \mathrm{~s}$. The particle filters have 5000 (PF1) and 10000 (PF2) particles. EKF is the extended Kalman filter. It is the product of background noise power and measurement bias on the $y$-axis. 


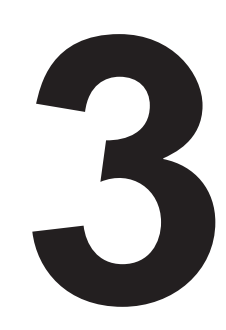

\section{Cellular Radio Communication}

A requirement for applying math to wireless communication systems is obviously a way of mathematically describing how a signal changes as it travels through the air. Since the exact behaviour of the propagation channel is far too complex to be described exactly, a model is used. The first section of this chapter describes how the propagation channel is modeled. Besides the propagation channel, two other important parts of a radio system are the transmitter and the receiver. Section 3.2 describes the generic parts of these two components.

For many reasons, one being that the bandwidth available for radio communication is limited and therefore expensive, the radio spectrum must be efficiently utilized. Methods for sharing the available bandwidth are presented in Section 3.3. Because of this sharing, users will interfere with each other. However, if they can be spatially separated, a user will share the available bandwidth with less users. This is one of the ideas behind cellular radio networks. Theory regarding cellular radio networks is further explored in Section 3.4. There is a number of expectations on a radio system. What these expectations are depends on what kind of relation you have with the system. An attempt to characterize the performance of the system is done in Section 3.5. In order to utilize available resources in an efficient manner, radio resource management algorithms are used. In Section 3.6 fundamental radio resource management algorithms are mentioned and their purpose explained.

Exactly how these algorithms are implemented is a choice of the individual system manufacturers. However, successful operation over manufacture borders requires standardization, both in terms of radio network architecture and in protocols between for example transmitters and receivers. The last section of this chapter presents details of the architecture of a WCDMA system and, for the present work interesting, parts of the current standard. 


\subsection{Radio Wave Propagation}

A signal propagating through the air is subject to attenuation. Given perfect knowledge of the surrounding environment, this attenuation can be calculated using Maxwell's equations. This, of course, is not practically feasible for many reasons. Therefore, a simplified version of the reality, a model, is used. A criteria for the model is that it will provide a statistically correct description of the attenuation. Instead of modeling the attenuation, its inverse, power gain, is often modeled. The received signal power can comprehensively be expressed as transmitted power times the power gain. The model is often separated into three components. The product of these three is the power gain

$$
g=g_{p} g_{s} g_{m p}<1
$$

where $g_{p}$ represents path gain ${ }^{1}, g_{s}$ shadow fading and $g_{m p}$ multipath fading. These three components are explained in a bit more detail below.

Path loss is the long term attenuation caused by the distance between transmitter and receiver. Path loss is the dominating factor in for example satellite communications. It is usually modeled as

$$
g_{p}=C_{p} r^{-\alpha},
$$

where $C_{p}$ is a constant which depends on the gain at the receiving antenna and the wavelength of the radio signals, $r$ is the distance between transmitter and receiver and $\alpha$ is a radio environment dependent, propagation exponent ranging from 2 (free space propagation close to the antenna) to 5.5 (far from the antenna in a very dense urban environment). This model, with terrain dependent $\alpha$ and $C_{p}$, was verified by Okumura et al. (1968) and Hata (1980). In cellular radio systems, $\alpha$ is usually taken equal to 4 (Gilhousen et al., 1991).

Shadow fading is due to large obstacles in the radio environment, objects which may absorb the radio wave. This part of the power gain is not, unlike the path loss, strictly increasing with the distance. Shadow fading is usually modeled using a log-normal distribution (Hata, 1980; Okumura et al., 1968)

$$
g_{s}=10^{\xi / 10}, \xi \in \mathcal{N}\left(0, \sigma_{s}\right) .
$$

This model assumes that the user is standing still and thus experiences the same shadow fading over time. A user moving around in the environment will experience time varying shadow fading. The correlation between two consecutive samples of the shadow fading depends on how fast the user is moving. Gudmundson (1991) proposes a model where the correlation is expressed using a relation between the user's speed, $v$, and a correlation distance, $d_{0}$. This distance is chosen together with an additional constant $\epsilon_{D}$ such that the correlation between the shadow fading at two points separated a distance $d_{0}$ should be

\footnotetext{
${ }^{1}$ The transmitting antenna's gain and performance of the algorithms in the receiver can also be incorporated in the path gain.
} 
$\epsilon_{D}$. The dependence between consecutive samples is then implemented by filtering the $\xi$-values through a first order low pass filter with a pole at $\epsilon_{D}^{\frac{v T}{d_{0}}}$

$$
y(t)=\frac{\left(1-\epsilon_{D}^{\frac{v T}{d_{0}}}\right) q}{q-\epsilon_{D}^{\frac{v T}{d_{0}}}} \xi(t), g_{s}=10^{y / 10}
$$

The constant $T$ is the sampling time used, $t=1,2, \ldots$ represents the discrete time instants and $q$ is the shift operator (e.g., $q^{-1} \xi(t)=\xi(t-1)$ ).

Multipath fading is caused by signals being reflected on obstacles in the radio environment. The reflections cause a signal to be received in several copies. Since these copies may arrive at different times and with different strength, they interfere either constructively or destructively. Multipath fading depends on the user's position relative the surrounding environment. Thus one position does not have a time constant multipath fading due to a time varying radio environment. This contribution can change very rapidly, which is why it is also called fast fading. Further details on multi path fading can be found in Sklar (1997). The changes in the multi path fading produces deep fades in the total power gain, but the multi path fading gain can occasionally be larger than $1(0 \mathrm{~dB})$. Multipath fading also causes local deep fades in the frequency spectrum. In case of narrow

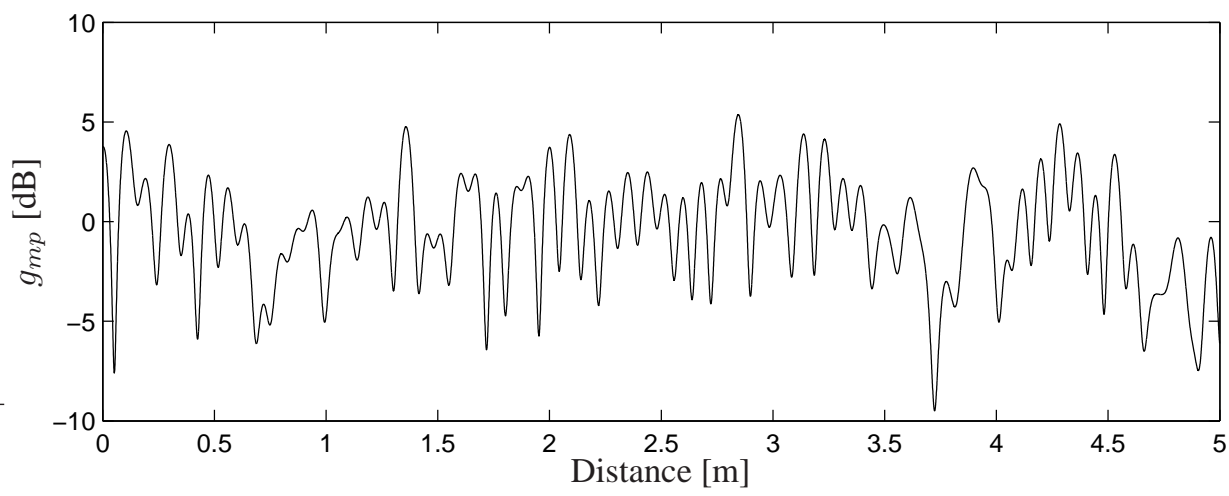

Figure 3.1: Example of the multipath fading part of the total power gain after the rake receiver, when adopting the characteristics given by $3 G P P$ Typical Urban multipath model, (3GPP, 2000c).

band communication this can be devastating.

One way of decreasing the variations in experienced power gain which the multipath fading causes is to use a rake receiver. A rake receiver estimates the relative delay of separate signal copies (rays). The information from different rays can then be combined providing a more stable total power gain after the rake receiver. Figure 3.1 illustrates the multipath fading gain after the rake receiver. 


\subsection{Radio Communication Systems}

Figure 3.2 shows the generic parts of a radio system. A message given to the source encoder can be in practically any format, such as a text file, a picture or speech. The source encoder converts this information into a string of bits.

These bits are then given to a channel encoder. A channel encoder adds redundancy bits, which in the receiver will be used to correct errors induced between sender and receiver. Both information bits and redundancy bits are then used to modulate a carrier signal. This process produces a high frequency signal which is suited for transmission over the air interface.

At the other end, on the receiver side, things are basically done in the opposite direction. However, algorithms here are much more complicated. For example, demodulation usually requires accurate synchronization between receiver and sender. The channel decoder uses the redundancy bits introduced by the channel encoder to detect and possibly correct bit errors. Finally, the source decoder converts the bits into the form of the original information. In order to provide a certain service to the users, the system has to

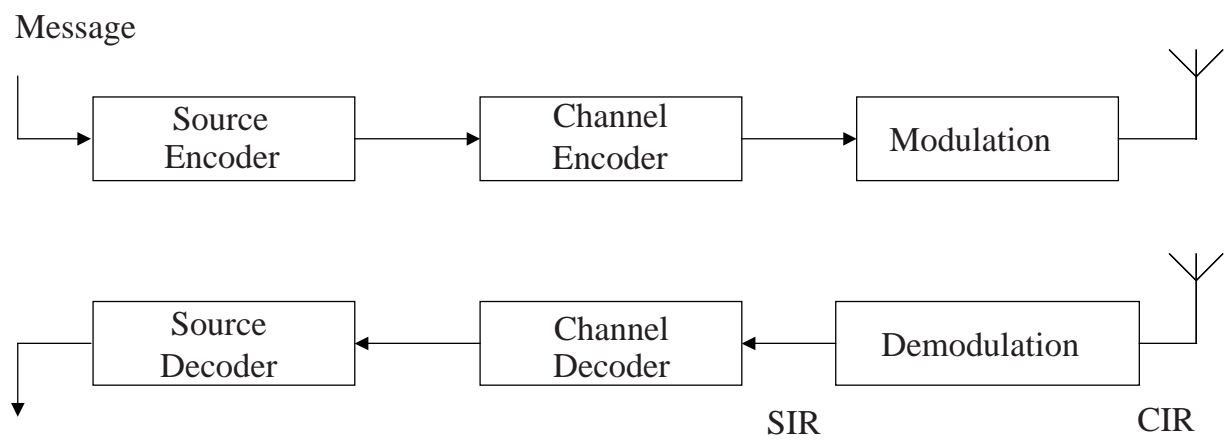

Estimated Message

Figure 3.2: The generic parts of a radio system.

provide each user with a received signal-to-interference ratio (SIR). A user's SIR is the ratio between the received power of the user's signal and the interference power. The interference power consists of the background noise power, $N$ and the signal power from all other users currently transmitting using the same frequency band (see Section 3.3). SIR is closely related to the more generally known signal-to-noise ratio. The difference lies in the fact that SIR considers the actual noise power, i.e., not just background noise but also noise originating from other users. Another user quality related quantity is a user's carrier-to-interference ratio (CIR), denoted $\gamma_{i}$. This is a measure of the power of the signal received from the user versus the interfering noise power, when measured at the receiving antenna. Thus, CIR is measured in the radio frequency band and SIR is measured in the base band, see Figure 3.2. 


\subsection{Multiple Access}

Because of limited availability of radio spectrum, it has to be shared between several users. This is done using some sort of multiple access technique. Regardless of which algorithm is used, the multiple access is implemented as a part of the modulation and demodulation of Figure 3.2. Below is a description of the three most common techniques, divided into two groups based on whether they use orthogonal signals or not.

\subsubsection{Orthogonal Signals}

A rather simple, but yet in many areas wide spread, technique is called Frequency Division Multiple Access (FDMA). The idea here is simply to split the available radio bandwidth into a number of (possibly differently sized) parts and assign each user one part. A publicly known example of FDMA is radio broadcasting. A drawback when using FDMA is that each user is limited to a (narrow) frequency band. Each user is stuck at using his assigned frequencies, even if there is locally heavy interference on this frequency band or the power gain is locally exceptionally low on these frequencies due to multipath fading.

The second generic technique, Time Division Multiple Access (TDMA), splits the radio spectrum in time, instead of in frequency. This technique requires precise synchronization between all users (which in some areas and applications even means taking the propagation time into account). An example of where TDMA is used is when several users share the same walkie talkie system. When using TDMA, each user is momentarily allocated the system's entire available frequency band. This means transmission over a larger bandwidth, and therefore less sensitivity to local narrow band interference.

The signals in the above mentioned techniques are what is sometimes referred to as orthogonal-signals. Ideally the users do not interfere with each other.

\subsubsection{Nonorthogonal Signals}

The third technique, Code Division Multiple Access (CDMA), uses non-orthogonalsignals. Using this technique, the users transmit independently of each other, using the same frequency. As this thesis is constrained to systems using CDMA, this technique for sharing resources will be explained in a bit more detail.

The idea behind CDMA is that each user is assigned an individual spreading code. The spreading code consists of a number of chips, each chip is either 1 or -1 . The number of chips per second, the chip rate, is higher than or equal to the symbol rate at which the user intends to send the information. The ratio between chip rate and symbol rate is called processing gain, $P G \geq 1$. Because of the processing gain, each user needs a CIR which is a factor $P G$ lower than would be required in for example FDMA to maintain the same SIR. It is the ability to use such a low CIR that has made CDMA popular in military applications, the transmitted signal can easily be hidden in the background noise and detecting it requires knowledge of the spreading code. In cellular applications however, the interference power is far higher using CDMA compared to the two other multiple access techniques shown here due to the concurrent transmission of several users on the same frequency band. Thus the required transmission power is still not decimated a factor $P G$. Due to the spreading, which is done in time domain (see Figure 3.3), the modulating 


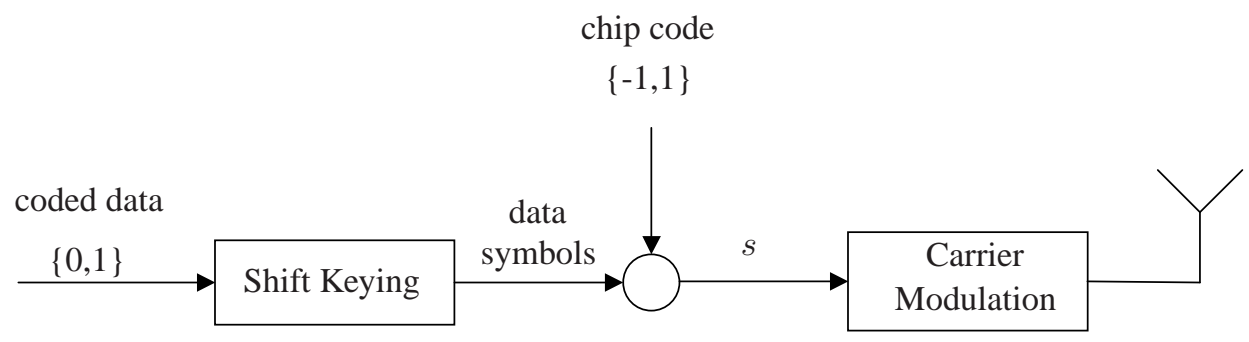

Figure 3.3: The basic blocks of the modulator in a CDMA system.

signal, $s$, has a bandwidth which is a factor $P G$ larger. With an increased bandwidth the transmitted signal is less sensitive to multipath fading. The inherent usage of a large bandwidth makes CDMA more spectrum efficient than the orthogonal signal techniques.

\subsection{Cellular Radio Networks}

Because of the attenuation between user and the access point providing the service, a too large distance implies an impairment in service and in the extreme case lack of connection due to limited transmission power. Hence, the distance has to be kept short.

By dividing the entire service area into a number of cells, each served by an access point which in this case is referred to as a base station, the attenuation can be kept at moderate levels yielding lower requirements on transmission powers. The attenuation's dependence on distance is thus utilized here since users far from each other will create negligible mutual interference if they belong to different cells. A system providing radio service over an area divided into several cells is called a cellular radio network.

The radio environment, expected user density and type of traffic should be considered when choosing the size of the cells. In an area where several users are expected to use high bit-rate-services simultaneously, the cells are chosen to be smaller. Typical cell radii are between a couple of hundred meters (in a dense urban environment) to several tenths of kilometers (in rural areas). In practice, a cell's size is defined by the location of the users connected to the cell. A user chooses cell based on the pilot signal from different base stations; the user applies for a connection to the base station with the strongest pilot signal. Therefore, since the transmission power of the pilot signal is variable, a cell can actually be resized dynamically during operation in order to adapt to changing user densities. This technique is referred to as cell breathing (Jalali, 1998).

In radio systems in general one denotes the direction from user to the access point (i.e., base station in the case of cellular networks) as uplink and conversely the opposite direction as downlink.

The uplink performance of a cellular radio network is highly dependent on the power gains between each user and the base stations. A comprehensive way of representing these power gains is through the (time varying) power gain matrix. 
Definition 3.1 (Power Gain Matrix). The power gain matrix of a system with $M$ users and $B$ base stations is

$$
G(t)=\left(\begin{array}{ccc}
g_{1,1}(t) & \cdots & g_{1, B}(t) \\
\vdots & \ddots & \vdots \\
g_{M, 1}(t) & \cdots & g_{M, B}(t)
\end{array}\right)
$$

where $g_{i, j}(t)$ is the power gain at time $t$ between user $i$ and the base station serving cell $j$.

The elements of the power gain matrix representing downlink are in general different from the corresponding ones in the power gain matrix representing the uplink.

Using the above notation for power gain between users and base stations, the uplink CIR user $i$ experiences in cell $j$ is defined as

$$
\gamma_{i, j}(t) \triangleq \frac{C_{i, j}(t)}{I_{j}^{t o t}(t)-C_{i, j}(t)}=\frac{p_{i}(t) g_{i, j}(t)}{N_{j}(t)+\sum_{l \neq i} p_{l}(t) g_{l, j}(t)},
$$

where $I_{j}^{t o t}(t)$ is the total interference power in base station $j, p_{i}(t)$ is user $i$ 's individual transmission power, $C_{i, j}(t)$ is the received carrier power of user $i$ in base station $j$ and $N_{j}(t)$ is the background noise in cell $j$, all at time $t$. The sum is over all users using the same frequency band. A similar ratio is the carrier-to-total-interference ratio (CTIR), $\beta$. The CTIR user $i$ experiences in cell $j$ is defined as

$$
\beta_{i, j}(t) \triangleq \frac{C_{i, j}(t)}{I_{j}^{t o t}(t)}=\frac{\gamma_{i, j}(t)}{1+\gamma_{i, j}(t)}<1 .
$$

The performance of a radio network is related to the CIR it can provide to the users. According to (3.3), the system performance is therefore dependent on the amount of induced interference from other users. Hence, it is of utmost importance to maintain a suitable transmission power at all times, a transmission power such that the required CIR is maintained while not inducing excessive interference to other users. Because of this, a fundamental mechanism of a cellular radio network is power control. Power control regularly decides what transmission power commands to send to each transmitter in the network (mobile phones and base stations). The intercell interference, which is due to the fact that all cells use the same frequencies, makes the power control problem much harder. However, since all cells share the same frequencies in a CDMA cellular network, there are no longer any hard frequency allocation problems to solve. In for example the second generation system used in Europe, Global System for Mobile Communications (GSM), huge amounts of efforts have been put into solving the complex optimization problem of allocating frequencies to different cells.

As a user moves from one cell to another, the power gain to the base station controlling the cell the user is connected to will decrease while the power gain to the neighbouring cell will increase. It is therefore natural to, at some point, change the cell the user is connected to. This maneuver is called handover. Since communication takes place on the same frequency in all cells of a CDMA cellular system, a user can actually be connected to several cells at a time. So, signals to/from a user located in between two or more cells 
can be send/received in several nearby located base stations. A user connected to several cells is said to be in soft handover. The cells that user $i$ is connected to is called the active set, denoted $K_{i}$. Signals can be received using maximum ratio combining or selection combining. Maximum ratio combining utilizes correlation between the received signals making the combined CIR equal to the separately received CIR values,

$$
\gamma_{i}=\sum_{k \in K_{i}} \gamma_{i, k}
$$

Selection combining, on the other hand, means that the combined CIR is simply the maximum CIR,

$$
\gamma_{i}=\max _{k \in K_{i}} \gamma_{i, k}
$$

Since selection combining implies disregarding information, it is less efficient than maximum ratio combining. The notation introduced so far is summarized in Figure 2.1.

In practice, the users' signals can not be decoded entirely correct. All users will therefore experience a degree of self interference, i.e., a part of a user's own signal will be experienced as interference. The fraction of the own received signal power that can not be subtracted in the denominator of (3.3) is called the self interference factor, $\alpha$. The relation between a user's CTIR, $\beta$, the user's perceived CIR, $\gamma$, and the self interference factor is

$$
\gamma=\frac{C}{I^{t o t}-(1-\alpha) C}=\frac{\beta}{1-(1-\alpha) \beta} .
$$

Since $\gamma$ tends to $1 / \alpha$ as $\beta$ tends to one, a nonzero self interference factor decreases the system performance and puts a limit on the maximum CIR that a user can perceive.

\section{Example 3.1: Intercell Interference}

Consider a system with two users and two cells. The power gain matrix is

$$
G=\left(\begin{array}{ll}
g_{1,1} & g_{1,2} \\
g_{2,1} & g_{2,2}
\end{array}\right)
$$

User 1 is solely connected to base station one while user two is initially not connected to the system at all. User one has a service requiring a carrier-to-interference ratio of $\gamma_{1}$ if the self-interference can be neglected. User two's transmission power is

$$
\gamma_{1}=\frac{p_{1} g_{1,1}}{N} \Leftrightarrow p_{1}=\frac{\gamma_{1} N}{g_{1,1}},
$$

where $N$ is the background noise power common to both cells. If user two is provided another type of service requiring a carrier-to-interference ratio of $\gamma_{2}$ through a connection with base station two, the required transmission powers satisfy the following system of equations

$$
\left\{\begin{array}{rl}
\gamma_{1} & =\frac{p_{1} g_{1,1}}{N+p_{2} g_{2,1}} \\
\gamma_{2} & =\frac{p_{2} g_{2,2}}{N+p_{1} g_{1,2}}
\end{array} .\right.
$$

Solving for $P=\left(p_{1} p_{2}\right)^{T}$ yields

$$
P=\left(\begin{array}{cc}
g_{1,1} & -\gamma_{1} g_{2,1} \\
-\gamma_{2} g_{1,2} & g_{2,2}
\end{array}\right)^{-1}\left(\begin{array}{l}
\gamma_{1} \\
\gamma_{2}
\end{array}\right) N
$$


Explicitly,

$$
\begin{aligned}
p_{1} & =\gamma_{1} \frac{g_{2,2}+\gamma_{2} g_{2,1}}{g_{2,2} g_{1,1}-\gamma_{1} \gamma_{2} g_{1,2} g_{2,1}} N>\frac{\gamma_{1}}{g_{1,1}} N \\
p_{2} & =\gamma_{2} \frac{g_{1,1}+\gamma_{1} g_{1,2}}{g_{1,1} g_{2,2}-\gamma_{2} \gamma_{1} g_{2,1} g_{1,2}} N .
\end{aligned}
$$

Thus, user 1's transmission power is affected by the decision to admit another user, even if it is in a neighbouring cell. The factor by which user 1's transmission power is increased depends on both $\gamma_{1}$ and $\gamma_{2}$.

User 1's increase in transmission power in the above example is to compensate for the increased intercell interference power.

\subsection{System Performance}

The performance of the system is quite hard to measure. First of all one needs to define what we expect from the system. This expectation clearly depends on what kind of user you are; subscriber or operator.

A subscriber typically expects to have a high probability of being allowed to use the system at any given time. This defines a quantity called Grade of Service (GoS). Once connected, the subscriber expects to experience some sort of quality of service (QoS) which is a truly subjective measure. For example, in the case of a speech service, the subscriber expects to hear the other person reasonably well, or in the case of a data-service, that the connection is fast enough without a too long delay. A connected subscriber expects to be provided services without being disconnected, something that is considered far worse than not getting a connection at all. These different measures, just from the subscribers point of view, make defining the system performance a hard task.

The operator, who owns and manages the system, also puts expectations on the system. These can for example be total throughput. However, maximizing the total throughput often comes with a degrade in individual subscriber satisfaction, which then, in the long run, means less customers for the operator.

This leads to a discussion of which policy to use, i.e., how to prioritize subscribers and what service to give them. The result is of course a trade off between individual subscriber satisfaction and maximum utilization of the resources.

Different choices of how resources are assigned to users will be compared in this thesis. For this end, a definition of capacity motivated by Shannon's theorem on the maximum channel capacity (Wozencraft and Jacobs, 1965, page 520) is made below.

Definition 3.2 (Capacity). The capacity is

$$
C \triangleq \sum_{i} \log _{2}\left(1+\gamma_{i}\right)
$$

where the sum is taken over a set of users.

The set of users considered in the definition depends on whether it is the capacity of an entire system consisting of several base stations, a single base station or just a single 


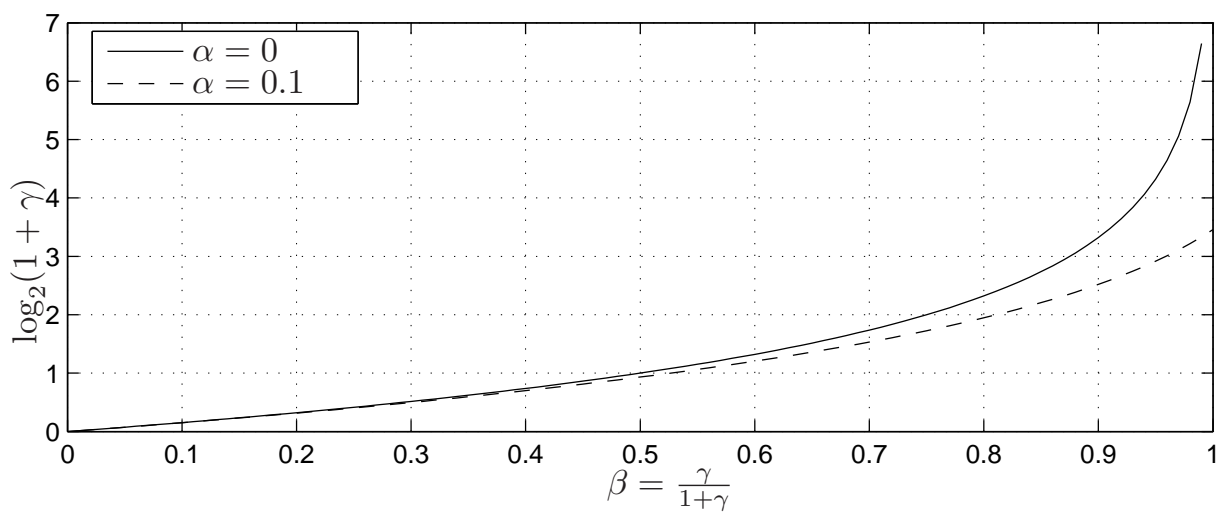

Figure 3.4: Relation between CTIR, $\beta$, and capacity, parameterized by the self interference factor $\alpha$. The capacity loss due to self interfernce is negligible for low CTIR levels.

user connection. Figure 3.4 shows the relation between a single user's capacity and CTIR for two different choices of the self interference factor $\alpha$. Figure 3.4 implies that the self interference factor only has a practical interest for high CTIR values. The self interference factor will therefore generally be omitted in the this thesis.

\subsection{Radio Resource Management Algorithms}

Obviously the ability of a system to provide service is limited. However, it is not as obvious in what way it is limited. An always present trade off is the one between capacity, quality and coverage. It is desirable to provide service to as many subscribers as possible at any given time. Allocating one subscriber a higher data rate or lower bit error probability means that his received signal power will be higher and thus higher interference to other subscribers is introduced. This means that less users can be accommodated into the system. Furthermore, since the mobiles' transmission powers are limited, higher interference power in the base stations means less coverage.

The choice of which subscribers to admit and what service they will be provided is a matter of policy. The choices which repeatedly have to be made in order to follow this policy are done by Radio Resource Management (RRM) algorithms. There is a wide range of RRM algorithms, starting from algorithms making decisions several times each millisecond such as fast power control to more long term decisions such as admission control which considers time periods of whole sessions (e.g., expected length of a phone call).

\subsubsection{Power Control}

Power control is used to set the users' transmission power such that they experience the block error rate (BLER) they have been guaranteed by other RRM algorithms, while not inducing unnecessary interference power into the system. For uplink capacity reasons 
among others, users' transmission powers have to be kept to a minimum while still providing satisfactory user individual SIR. Power control is often implemented as two loops, inner and outer loop.

The inner loop, also known as fast power control, adjusts each user's transmission power such that the CIR equals a user individual target CIR, $\gamma^{t g t}$. The fast power control is often based on decision feedback

$$
\begin{aligned}
\text { Receiver: } s_{i}(t) & =\operatorname{sign}\left(\gamma_{i}^{t g t}(t)-\gamma_{i}(t)\right) \\
\text { Transmitter: } p_{i}(t) & =p_{i}(t)+\Delta s_{i}(t-n),
\end{aligned}
$$

where $\Delta$ is a fixed value by which the transmission power is changed every time instant $t$ and $n$ is a delay caused by measurement times and discrete transmission instants. This is a well known technique for power control with limited feedback bandwidth. A fast update rate enables tracking of the multipath fading at low velocities. In for example WCDMA the fast power control updates each user's transmission power 1500 times a second. However, a fast update rate also induces a few problems. Perhaps most obvious is the amount of signaling required between each user and the base stations, but most severe is the ability to rapidly raise the transmission powers to levels which may jeopardize system stability.

The outer power control loop, adjusts each user's target CIR. Here, quality is simply converted into a certain target BLER. The outer loop is necessary because an average CIR can not be directly mapped to a BLER, i.e., if the carrier-to-interference ratio fluctuates too much the received BLER increases. The outer loop adjusts each user's target CIR at a rate of somewhere around 10 to $100 \mathrm{~Hz}$.

The power control implementation is thus a cascade control system. The inner loop is the fast power control operating between base station and mobile to adjust the mobile transmission power. The outer loop adjusts the target CIR for the inner loop such that the resulting BLER is neither too high or too low. The outer loop operates only in the core network.

\subsubsection{Load Control}

If the system has too many users, power control can not find transmission powers such that all users achieve their target SIR, not even if they had access to infinite transmission power. Too many users in the system simultaneously results in what is usually referred to as party effect, which can be understood by studying (3.3). An increase in the interference power, results in power control demanding an increase in transmission power. Increased transmission power means higher interference power, and so on. If a there exists finite transmission powers providing required carrier-to-interference ratio to all users in the network, the power control problem is said to be feasible, otherwise it is infeasible (Zander, 1993). To avoid too high transmission powers caused by too many users in the system, the load of the system needs to be controlled. Therefore, the system has a number of load controlling resource management algorithms which operate on different time scales, all much slower than the fast power control. Below is a brief explanation of some load controlling radio resource management algorithms.

- Admission Control is basically a door watchman. In order to send information, a user needs a session. A new user is given a session only if the system is ex- 
pected to cope with that user's request for system resources throughout the entire session. Note that, being granted a session does not always imply continuous access to the network resources, see Packet Scheduling and Channel Switching below. Admission control can for example study uplink received power as load measure, see (Choi and Bahk, 2001; Dimitriou et al., 2000; Holma and Laakso, 1999; Huang and Yates, 1996; Ishikawa and Umeda, 1997; Kuri and Mermelstein, 1999; Lei et al., 1999; Outes et al., 2001; Timus and Pettersson, 2001).

- Packet Scheduling is the task of selecting which packet users that will be allowed to send. This is typically done on a time scale of about two to twenty milliseconds. This algorithm only applies to packet users, i.e., not for speech users. Laakso et al. (1998) formulate the packet scheduling problem as an optimization problem. A special mode of WCDMA in which higher data rates can be accomplished at the price of less fairness is discussed by Kolding et al. (2002).

- Channel Switching handles users who already have a session. During the session, a user's channel conditions and the load of the base stations change. To adapt to these changes, channel switching increases and decreases the users' maximum transmission rate. A lower transmission rate means a lower target SIR. Channel switching in WCDMA is studied by Gyung-Ho and Dong-Ho (2000).

- Congestion Control can be compared to a bouncer at a restaurant. In more extreme cases, when neither Channel Switching or Packet Scheduling can take the system from an overload situation back to the target load, congestion control uses actions which are stronger than the previous algorithms. Examples of possible actions are

- denying users' requests for increased transmission power in the downlink

- move some users to an alternative network, e.g., GSM

- down switching speech users to a worse speech quality or even drop some real time users.

Being disconnected is usually consider far more annoying for a user than never being connected at all. Hence disconnection is strongly avoided by the system.

- Link Admission is the algorithm considering users' requests for augmenting their active set with another cell. Important to note here is that the link admission decision depends on the downlink only. The reason being that an extra link means higher transmission power for the base station serving the new cell. In the uplink, on the other hand, an additional link does not imply increased transmission power. Therefore, the uplink can only gain from more soft handover links. 


\subsection{WCDMA}

The architecture of a WCDMA system contains a number of levels. Starting from cell level, the service in each cell is provided by a base station, see Figure 3.5. A base station hosts all lower level (link level) algorithms such as fast power control. The figure also

Outside World

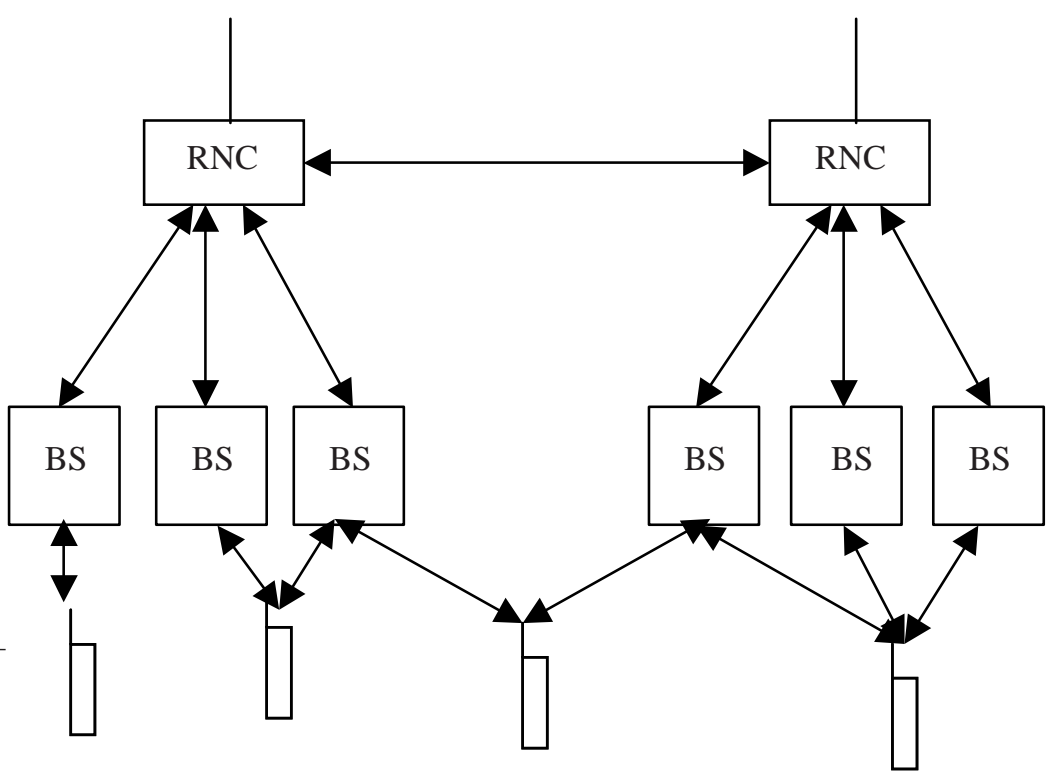

Figure 3.5: The architecture of a WCDMA system. The outside world is connected to the network at Radio Network Controllers (RNC). Each RNC is connected to a number of base stations (BS). Lower down in the architecture, each base station is connected to several mobiles.

shows how signals from different base stations are combined further up in the hierarchy in a Radio Network Controller (RNC). A radio network controller receives information gathered in several cells, even from cells controlled by other RNCs. This, more complete, information about the system as a whole can be used by radio resource management algorithms in general and relative load estimates in particular.

A common standard is a requirement for product interaction over manufacture borders. The committee specifying the WCDMA standard is called Third Generation Partnership Project (3GPP) ${ }^{2}$. They specify parameters regarding measurement accuracies, possible data-rate configurations and power control frequency to mention a few. In this section we will only mention the small part of the standardization process concerning this work. To enable reasonable well tracking of the multipath fading while not using too

\footnotetext{
${ }^{2}$ http://www.3gpp.org
} 
much bandwidth, the power control feedback has a bandwidth of 1500 bps. The standard states that the system should contain RNCs. Each RNC controls a number of base stations. Since the RNC has knowledge of the situation in a far larger area than a single base station does, many of the load controlling schemes reside here such as admission and congestion control.

An addition to the 3GPP standard is the enhanced uplink (EUL) (3GPP, 2005). The idea with this addition is to move much of the load controlling algorithms operating on a fast time scale from the RNC to the base stations. This decentralization will enable faster load control. The base stations may also have more accurate information on the current radio conditions in the base station's immediate surroundings.

Each base station may control several cells in WCDMA. A user is said to be in softer handover between cells in the active set controlled by one base station and in soft handover between cells in the active set controlled by different base stations. When using softer handover, the signals are combined using maximum ratio combining and when using soft handover, selection combining is used. The reason for this is simple, maximum ratio combining requires information about the correlation between the signals as the combining is done before decoding. Selection combining, on the other hand, is done after decoding and therefore only needs a reliability tag such as the estimated received CIR for the separately received signals. Hence, soft handover yields selection combining and softer handover maximum ratio combining. Figure 3.6 explains how the signals are combined at different levels of the hierarchy.

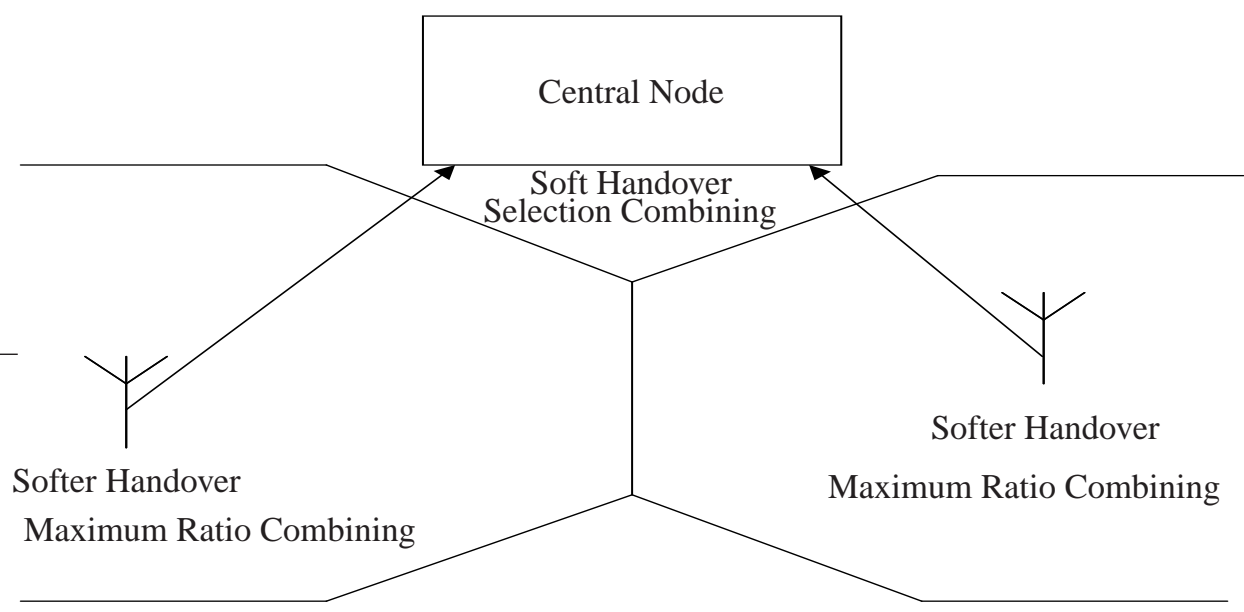

Figure 3.6: The combination of soft and softer handover in a WCDMA system. 


\section{Characterizing Uplink Load}

The purpose of this chapter is to characterize uplink load and introduce various ways of looking and approximating it. Inherent properties of CDMA yield that the uplink is interference limited, i.e., the system performance is limited by the amount of received interference power. A formal definition of uplink relative load is given Section 4.1. Since an interference limited system is considered, the definition relates load to the amount of interference power received in the base stations.

That the uplink is interference limited also means that there is not a one-to-one relation between how loaded the system is and, for example, how many channels that are occupied. This makes it harder to determine the fraction of available resources that are currently in use, compared to systems with a fixed number of channels. Predicting the load is even harder, since that involves estimating the future received interference power.

Load measures in the literature can be divided into those considering hard resources and those based on soft resources. These two types of resources will be explained and compared in Section 4.1.

Another way of dividing the load measures is related to where they reside. Resource management algorithms can reside in a base station. An advantage with this is of course that a minimum of signaling is required if the decisions can be made on this intermediate level. Relative load estimates designed to reside in base stations are referred to as decentralized estimates. Theory related to such estimates is presented in Section 4.2.

A base station has no information about the situation in cells supported by other base stations. Thus, a decentralized estimate can not predict how resource management decisions will affect cells supported by other base stations. However, nodes at a higher level in the system's architecture have access to information gathered in several cells. This more complete information about the system as a whole can be used by load estimates at this level. Section 4.3 discusses centralized load measures, related to the entire system as opposed to the different base stations.

The theoretical total capacity, the pole capacity, is interesting to study for comparative 
purposes, even though it is not achievable in practice. Along the way, pole capacity will be discussed from the different angles presented here.

Section 4.4 concludes the chapter by discussing different aspects of the presented approaches to estimating the uplink load.

\subsection{System Load and Capacity}

A basic requirement for providing service to users is that there is sufficient power available to maintain an acceptable QoS. In the uplink this, among other things, means that the total received interference power in the base station must not be too high. The total received uplink interference power is

$$
I^{t o t}=N+\sum_{i=1}^{M} C_{i},
$$

where $N$ represents the part of the interference power which is not power controlled by our system. All contributions to the total interference power which originate from terrestrial non-power controlled sources, such as cellular systems operating at neighbouring frequencies and electronic equipment in for example cars, are thus embedded into $N$. Added to the background noise is the sum of the users' carrier powers, $C_{i}$ for user $i$. The sum is taken over all $M$ users in the entire network. In the literature, interference power is often related to background noise power through the uplink noise rise.

Definition 4.1 (Uplink Noise Rise). Uplink noise rise, $\Lambda$, is defined as the total uplink received interference power, $I^{t o t}$, divided by the background noise power, $N$, i.e.,

$$
\Lambda \triangleq \frac{I^{t o t}}{N} \text {. }
$$

Since the uplink noise rise is the constraining resource, and increasing the number of active users or the active users' quality results in a noise rise increase, there is a natural trade off between the number of users and quality. Furthermore, as the users have limited transmission power, a higher noise rise means reduced coverage. An always present trade off is therefore one between the number of users, quality and coverage. Perhaps not useful in practice, but still an educational model is

$$
\text { Quality + Number of users }+ \text { Coverage }=\text { Utilized resources. }
$$

The amount of available soft resources is unknown and time varying. An alternative is therefore to estimate the uplink relative load or uplink fractional load, $L$, i.e., the amount of currently utilized resources relative to the total amount of resources

$$
\text { Quality }+ \text { Number of users }+ \text { Coverage }=L \cdot \text { total resources. }
$$

Since performance of the system is related to the currently received interference power, a more formal definition of load should incorporate this property of the system. One way is to relate the useful received interference power to the total received interference power. Mathematically this can be expressed as

$$
L=\frac{\sum_{i=1}^{M} C_{i}}{N+\sum_{i=1}^{M} C_{i}}=\frac{I^{t o t}-N}{I^{\text {tot }}}=1-\frac{1}{\Lambda} .
$$


Below is a formal definition of load which is inspired what has been said above.

Definition 4.2 (Noise Rise Relative Load). The noise rise relative load of a CDMA cellular system is defined as

$$
L^{n r} \triangleq 1-\frac{1}{\Lambda}
$$

A rearrangement of the expression in the above definition yields the pole equation (Holma and Toskala, 2000)

$$
\Lambda=\frac{I^{t o t}}{N}=\frac{1}{1-L^{n r}} .
$$

The equation clearly shows that $L^{n r}=0$ implies $I^{t o t}=N$, i.e., an empty system with only background noise. As the noise rise relative load approaches one the system is operated close to the system's theoretical capacity, the pole capacity, and the interference power goes to infinity, see Figure 4.1.

\section{Example 4.1: Noise Rise Relative Load, Single Cell}

The total received interference power in a single cell scenario can be expressed as in (4.1). The QoS of each user is related to the carrier-to-total-interference ratio,

$$
\beta_{i}=\frac{C_{i}}{I^{t o t}}
$$

Solving for $C_{i}$ in (4.4) and inserting it into (4.1) yield

$$
I^{t o t}=N+\sum_{i=1}^{M} \beta_{i} I^{t o t} \Leftrightarrow \Lambda=\frac{I^{t o t}}{N}=\frac{1}{1-\sum_{i=1}^{M} \beta_{i}} .
$$

The equation has the same form as (4.3) and therefore according to Definition 4.2,

$$
L^{n r}=\sum_{i=1}^{M} \beta_{i}
$$

The noise rise relative load is clearly a function of the received interference power. In the multi cell case, the noise rise relative load is therefore not purely a function of how many users there are in the system, but also for example where the users are located in the radio environment. This means that the pole capacity in terms of e.g., number of users is in general both unknown and time varying. From the inherent requirement of a positive noise rise and (4.3) we can conclude that a feasible total resource allocation is associated with a noise rise relative load between zero and one. Example 4.1 shows that the capacity of a CDMA system with conventional receivers (i.e., not utilizing multiuser detection) is in fact interference limited because the interference from other connections limits the capacity. The opposite is a noise limited system . 


\section{Example 4.2: Noise Limited System}

Consider a system with one user in an isolated cell. The carrier-to-total-interference ratio is

$$
\beta=\frac{C}{I^{t o t}}=\frac{C}{N+C}<1,
$$

eliminating $C$ in this expression gives

$$
I^{t o t}=\frac{1}{1-\beta} N<\infty .
$$

The total interference power, $I^{\text {tot }}$ is finite for all possible $\beta$ since $\beta<1$. In a noise limited system, quality (data rate) is therefore limited by the amount of available transmission power.

According to (4.5), the number of users in an interference limited system is, even with unlimited transmission power, limited by the mutual interference power between the connections. Furthermore, the amount of additional interference power caused by admitting a new user depends on the current interference power in the system. Figure 4.1 illustrates the higher interference power contribution of an admitted user at high load, compared to that at low load. In Figure 4.1, $\Delta L^{n r}$ is associated with the amount of noise rise relative load a user contributes with. For example in a scenario with an isolated cell and perfect power control, $\Delta L^{n r}=\beta^{t g t}$ for the admitted user according to (4.6). The constant $\beta^{t g t}$ is the CTIR that the power control aims at.

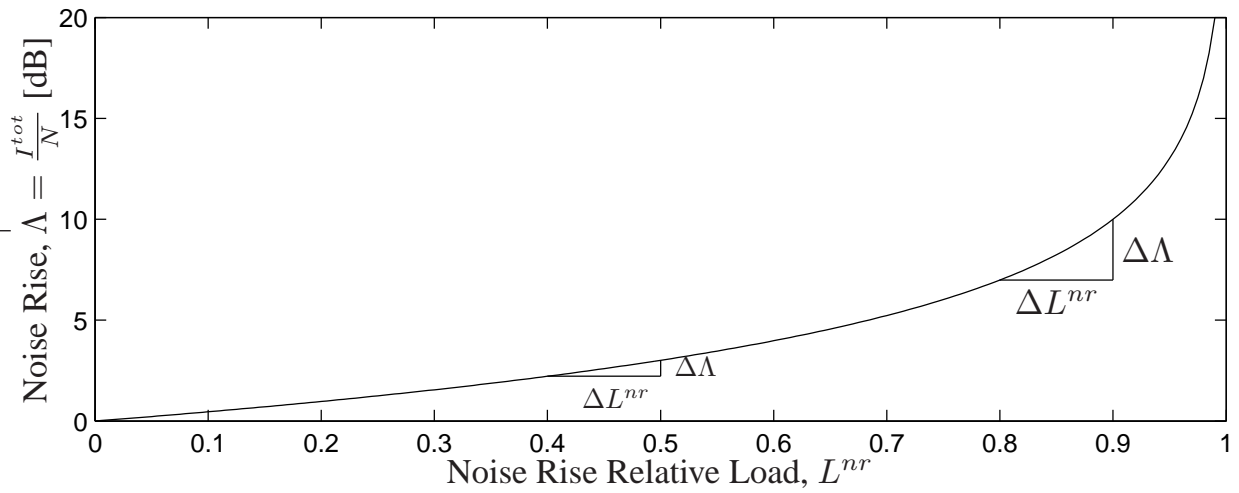

Figure 4.1: The nonlinear relation between noise rise relative load, $L^{n r}$, and noise rise, $\Lambda . \Delta L^{n r}$ could be the increase caused by admitting another user. This gives different noise rise contribution, $\Delta \Lambda$, at different load levels.

A traditional definition of relative load, which is suitable for FDMA and TDMA systems, is the number of currently used channels over total number of channels, i.e.,

$$
L^{\text {trad }} \triangleq \frac{M}{M^{\max }} \text {. }
$$

A capacity defined as a fixed maximum number of channels is an example of a hard capacity. The hard capacity of a system is fixed and known, unlike the soft capacity. The 
soft capacity can only be achieved when a soft resource is studied in the resource management algorithms. Uplink noise rise is an example of a soft resource since it depends on time varying variables such as the power gains the users experience. As the uplink of a CDMA cellular system is limited by this spatial resource, the uplink's capacity depends on the situation in several cells. If the load is low in surrounding cells, little interference power is received from these cells. This results in an increased capacity in the own cell compared to when the surrounding cells are more loaded. A centralized resource management algorithm based on a soft resource can, unlike a decentralized algorithm studying a hard resource, utilize this additional capacity. Example 4.4 at the end of the chapter studies this difference.

In the cases where the soft capacity equals the hard capacity, the traditional definition of relative load coincides with Definition 4.2. Consider a single cell situation in which the only service available is characterized by a target carrier-to-total-interference ratio of $\beta_{0}$. By using (4.6), the pole capacity can then be calculated as ( $L^{n r}=1$ and assuming perfect power control, $\beta_{i}=\beta_{0}$, give the maximum number of users)

$$
1=\sum_{i=1}^{M^{\text {pole }}} \beta_{0}=M^{\text {pole }} \beta_{0} \Leftrightarrow M^{\text {max }}=M^{\text {pole }}=\frac{1}{\beta_{0}} .
$$

According to (4.6), the noise rise relative load in this scenario is merely the number of users times $\beta^{t g t}$. The noise rise relative load can thus be expressed as

$$
L^{n r}=M \beta_{0}=\frac{M}{1 / \beta_{0}}=\frac{M}{M^{\max }}=L^{t r a d} .
$$

Huang and Yates (1996) study a single service scenario with several cells. The total interference is therein divided into three parts; background noise, intracell interference, $I^{\text {own }}$, which is interference from users within the cell, and intercell interference, $I^{\text {other }}$, which is interference from users in other cells,

$$
I^{\text {tot }}=N+I^{\text {own }}+I^{\text {other }} .
$$

Since a single service scenario is studied, $I^{\text {other }}$ can be converted into a corresponding number of users connected to other base stations, $M^{\text {other }}=I^{\text {other }} / C$, where $C$ is the received power required to maintain $\beta_{0}$. This once again enables an expression corresponding to (4.7), only with $M$ substituted for $M^{\text {own }}+M^{\text {other }}$, where $M^{\text {own }}$ is the number of users connected to the own base station.

\subsection{Decentralized Load}

Some resource management algorithms act in a decentralized node, in which only local information is available. For example, the part of the interference power caused by users connected to another base station, $I^{\text {other }}$, depends on variables not known in the own base station. Therefore a decentralized estimate has to either measure it or somehow estimate it using local variables only. 


\subsubsection{Intercell-to-Intracell-Interference Factor}

One way of eliminating the intercell interference from (4.8) is to simply state that it is a nominal fraction, $f$, of the intracell interference, i.e.,

$$
I^{\text {other }}=f I^{\text {own }} \text {. }
$$

This is a natural assumption since an increase in interference power in one cell leaks to surrounding cells. Higher intercell interference power in surrounding cells causes users in these cells to use higher transmission power, yielding higher intercell interference power in the own cell. This effect would not be captured if $I^{\text {other }}$ is assumed constant.

Combining the above expression with (4.8) results in an expression for $I^{\text {tot }}$ that contains only local variables

$$
I^{\text {tot }}=N+(1+f) I^{\text {own }} .
$$

According to (4.4), the received power of user $i$ is $C_{i}=\beta_{i} I^{t o t}$. The interference power from users in the own cell, $I^{\text {own }}$, is simply the sum of these user individual carrier powers. An expression for the total interference power is therefore

$$
I^{t o t}=N+(1+f) \sum_{i=1}^{M^{\text {own }}} C_{i}=N+(1+f) \sum_{i=1}^{M^{\text {own }}} \beta_{i} I^{\text {tot }}
$$

Solving for $I^{\text {tot }}$ yields

$$
I^{\text {tot }}=\frac{N}{1-(1+f) \sum_{i=1}^{M^{\text {own }}} \beta_{i}} .
$$

Once again comparing with (4.3), we see that an approximation of the noise rise relative load in a multi cell scenario is

$$
L^{n r} \approx(1+f) \sum_{i=1}^{M^{\text {own }}} \beta_{i}
$$

A comparison with (4.6) shows that one should not consider cells as isolated, since this gives an underestimation of the noise rise relative load. This is perhaps obvious since considering cells as isolated corresponds to completely ignoring the intercell interference, $I^{\text {other }}$ in (4.8). Using this technique, the requirement for pole capacity that the noise rise relative load should equal one corresponds to

$$
\sum_{i=1}^{M^{\text {own }}} \beta_{i}=\frac{1}{1+f}
$$

Hence, an estimate of the pole capacity is put in terms of combined carrier-to-totalinterference ratio. In case of a single service scenario, an estimate of the maximum number of users would be

$$
M^{\max }=\left\lfloor\frac{1}{(1+f) \beta_{0}}\right\rfloor,
$$

where the brackets implies that the ratio should be rounded to the nearest lower integer. The intercell-to-intracell factor, $f$, is widely used throughout the literature. Boyer et al. 
(2001); Hiltunen and Binucci (2002); Holma and Laakso (1999); Ying et al. (2002); Zhang and Yue (2001) and Sanchez et al. (2002) use it in noise rise relative load expressions. A range in which $f$ is usually chosen is between 0.4 and 0.6 if uniformly distributed traffic is expected.

\subsubsection{Interference Power Measurements}

Another way of considering the entire interference power using only local information is to simply assume that it is measurable. As concluded in Section 4.1, the increase in interference power due to an admitted user depends on the interference level. Holma and Laakso (1999) use measurements of the total received interference power, $I^{\text {tot }}$, and approximates the additional interference power a new user would cause through derivatives of (4.3)

$$
\frac{\partial I^{t o t}}{\partial L^{n r}}=\frac{N}{\left(1-L^{n r}\right)^{2}}=I^{t o t} \frac{1}{1-L^{n r}} .
$$

An approximative expression for the interference power increase due to an additional load of $\Delta L^{n r}$ is then

$$
\Delta I^{t o t}=\frac{\partial I^{t o t}}{\partial L^{n r}} \Delta L^{n r}=I^{t o t} \frac{\Delta L^{n r}}{1-L_{0}^{n r}},
$$

where $L_{0}^{n r}$ is the noise rise relative load before admitting the new user. An alternative expression for the interference power increase is derived as the integrated difference in $I^{t o t}$

$$
\begin{aligned}
\Delta I^{t o t} & =\int_{L_{0}^{n r}}^{L_{0}^{n r}+\Delta L^{n r}} \frac{\partial I^{t o t}}{\partial L^{n r}} d L^{n r}= \\
\frac{I^{t o t}}{1-L_{0}^{n r}-\Delta L^{n r}} \Delta L^{n r} & =I^{t o t}\left(L_{0}^{n r}+\Delta L^{n r}\right)-I^{t o t}\left(L_{0}^{n r}\right),
\end{aligned}
$$

where $I^{t o t}\left(L^{n r}\right)$ is the total interference power when $L^{n r}$ is used in (4.3). The last equality is put there simply as a reminder that integrating a derivate is the same as taking the difference between the function values at the end points of the integration interval.

Motivated by the calculations leading to (4.6), $\Delta L^{n r}$ can be estimated as the new user's target CTIR. Using measurements of the total interference power inherently catches the variations in intercell interference power. However, it relies heavily on somewhat accurate measurements of the current uplink interference power.

\subsection{Centralized Load}

The estimates in Section 4.2 can not predict the effects a decision made in one cell will have on other cells. Users located close to the cell border are especially important. These users can occasionally introduce considerable interference power in other cells as the following example shows. 


\section{Example 4.3: Intercell Interference}

Study a prospective user in cell $k$. Power control will force him to use a transmission power $p_{i}$ satisfying

$$
C_{i, k}=p_{i} g_{i, k}=\beta_{i}^{t g t} I_{k}^{t o t} \Leftrightarrow p_{i}=\beta_{i}^{t g t} I_{k}^{t o t} \frac{1}{g_{i, k}} .
$$

User $i$ 's signal will be received in cell $j$ with a power of

$$
C_{i j}=p_{i} g_{i, j}=\beta_{i}^{t g t} I_{k}^{t o t} \frac{g_{i, j}}{g_{i, k}} .
$$

The received carrier power $C_{i j}$ will in cell $j$ be a part of the intercell interference. The magnitude of $C_{i j}$ depends on where the user is located. If user $i$ is close to the cell border, $g_{i, j}$ and $g_{i, k}$ will be of the same order and $C_{i j}$ will thus to a greater extent contribute to the noise rise relative load in cell $j$, compared to the case where user $i$ is close to base station $k$.

From a resource management point of view it is quite interesting to have an idea of how much load a user will actually induce in the own and surrounding cells. This, however, requires information which is gathered in several cells and an estimate of the noise rise relative load using this information would therefore have to reside in a more centralized site than a base station serving just one cell.

\subsubsection{Feasibility Relative Load}

Thus far, relative load has been related to received interference power. It can equally well be related to the users' transmission powers. All work in this section is done under an assumption that a user is power controlled in exactly one cell, i.e., soft handover is not utilized here. Zander (1993) studies solvability of the power control equation in the downlink. The basis for the work is the power control equation, which is derived below. Let user $i$ be power controlled solely from base station $j_{i}$. The $i$ :th position of the vector $j$ thus contains the number of the base station user $i$ is connected to. User $i$ 's downlink carrier-to-interference ratio can be expressed as

$$
\gamma_{i}=\frac{C_{i}}{I_{i}^{t o t}-C_{i}}=\frac{p_{i} g_{i, j_{i}}}{N_{i}+\sum_{\ell \neq i} p_{l} g_{i, j_{\ell}}}=\frac{p_{i}}{\frac{N_{i}}{g_{i, j_{i}}}+\sum_{\ell \neq i} p_{\ell} \frac{g_{i, j_{\ell}}}{g_{i, j_{i}}}} .
$$

Since this is the downlink, $p_{i}$ is the transmission power which information to user $i$ is sent with. $N_{i}$ is the background noise user $i$ experiences. Introduce the following matrix and vectors

$$
P(t) \triangleq\left[p_{i}(t)\right], \eta=\left[\eta_{i}\right] \triangleq\left[\frac{N_{i}}{g_{i, j_{i}}}\right], \bar{Z}=\left[\bar{z}_{i \ell}\right] \triangleq\left[\frac{g_{i, j_{\ell}}}{g_{i, j_{i}}}\right] \in \mathbb{R}^{M \times M} .
$$

In a single service system, i.e., all $\gamma_{i}=\gamma_{0}$, the above equation for all users can be put in matrix form

$$
P=\frac{\gamma_{0}}{1+\gamma_{0}}(\eta+\bar{Z} P)
$$


Clearly, it is interesting to study for which $\gamma_{0}$ this equation is solvable (with all $p_{i}>0$ ). Zander (1993) shows that, given knowledge of all power gains in the system, it is possible to determine the maximum achievable $\gamma_{0}$ in the noise free case, $\gamma_{0}^{*}$.

For convenience, introduce the notation

$$
\bar{\lambda}(A)=\max |\operatorname{eig}(A)|,
$$

for the spectral radius of a matrix $A$.

Theorem 4.1 (Zander, 1993)

Whenever the receiver noise is negligible, there exists, with probability one, a unique maximum achievable CIR-level

$$
\gamma_{0}^{*}=\sup \left\{\gamma_{0} \mid \exists P \geq 0: \gamma_{i} \geq \gamma_{0} \forall i\right\} .
$$

The maximum is given by

$$
\gamma_{0}^{*}=\frac{1}{\bar{\lambda}(\bar{Z})-1}
$$

The power vector $P^{*}$ achieving this maximum CIR-level is the eigenvector corresponding to $\bar{\lambda}(\bar{Z})$.

According to Theorem 4.1, simply choosing all users' $\gamma_{i}^{t g t}$ less than or equal to $\gamma_{0}^{*}$ guarantees existence of a positive solution to (4.11) in the single service case and in absence of noise, i.e., $N_{i}=0$. However, Zander (1993) also states that even in the noisy case, where $N_{i}>0$, the influence of background noise can be made arbitrarily small by scaling all users' transmission powers with a factor which is large enough.

The above theorem can be used to determine whether or not there is a base station transmission power vector $P$ such that all user achieves the maximum possible C/Irequirement. In a cellular system, it can sometimes be interesting to use a smaller $\gamma_{0}$ than $\gamma_{0}^{*}$. By formulating and solving a linear programming problem Zander (1993) shows the following theorem

\section{Theorem 4.2 (Zander, 1993)}

Whenever $\gamma_{0}<\gamma_{0}^{*}$, the power vector $P$ of least total (sum) power achieving the C/I-level $\gamma_{0}$ will be the solution to the following system of linear equations:

$$
\left(\frac{1+\gamma_{0}}{\gamma_{0}} E-\bar{Z}\right) P=\tilde{N} .
$$

where E denotes the identity matrix.

Thus, by combining Theorem 4.1 and Theorem 4.2, we can conclude that all positive $\gamma_{0}<\gamma_{0}^{*}$ are possible to achieve. As pointed out by Gunnarsson (2000), requiring all users' $\gamma_{i}^{t g t}<\gamma_{0}^{*}$ is a sufficient but not necessary condition in a multi service scenario. Thus a load measure based on e.g., the ratio between the maximum current $\gamma_{i}^{t g t}$ and $\gamma_{0}^{*}$ would be quite conservative. To handle multiple service, introduce the matrix $\Gamma^{t g t}$ which is a diagonal matrix with the users' target CIR in the diagonal,

$$
\Gamma^{t g t} \triangleq \operatorname{diag}\left(\gamma_{1}^{t g t}, \gamma_{2}^{t g t}, \ldots, \gamma_{M}^{t g t}\right) .
$$

Gunnarsson (2000) uses the above ideas to define feasibility relative load for multi service systems. 
Definition 4.3 (Gunnarsson, 2000). The downlink feasibility relative load is defined as

$$
L_{r}=\inf \left\{\mu \in \mathbb{R}: \frac{1}{\mu} \Gamma^{t g t} \text { is feasible }\right\} .
$$

A set of target CIR:s are said to be feasible if there is a solution to the resulting power control problem. Gunnarsson (2000) also presents the following theorem to find the downlink feasibility relative load in a system without soft handover.

Theorem 4.3 (Gunnarsson, 2000)

The feasibility relative load, $L_{r}$, of a system is

$$
L_{r}=\bar{\lambda}\left(\Gamma^{t g t}(\bar{Z}-E)\right) \text {. }
$$

In case of a feasible system, i.e., $L_{r}<1$, the transmission powers are

$$
P=\left(E-\Gamma^{t g t}(\bar{Z}-E)\right)^{-1} \Gamma^{t g t} \eta,
$$

where $\eta$ is a $M$-dimensional vector with the $i$ :th element equal to $N_{i} / g_{i, i}$.

By using the above theorem it is possible to show that the downlink feasibility relative load of a single service scenario is the ratio between the target CIR of the only service provided and $\gamma_{0}^{*}$ in a single service scenario,

$$
L_{r}=\bar{\lambda}\left(\Gamma^{t g t}(\bar{Z}-E)\right)=\bar{\lambda}\left(\gamma_{0} E(\bar{Z}-E)\right)=\gamma_{0}\left(\lambda^{*}-1\right)=\frac{\gamma_{0}}{\gamma_{0}^{*}} .
$$

This justifies Definition 4.3, given Theorem 4.1.

\subsubsection{Convergence of Power Control Algorithms}

Inspired by the presentation done by Hanly and Tse (1999), the uplink interference power in a multi-cell system is studied. Therein, user $i$ is power controlled solely from one cell, here denoted cell $j_{i}$. Consider a power control algorithm that at each time instant $t$ sets user $i$ 's transmission power according to

$$
p_{i}(t)=\gamma_{i}^{t g t}\left(\frac{N_{j_{i}}}{g_{i, j_{i}}}+\sum_{\ell \neq i} p_{\ell}(t-1) \frac{g_{\ell, j_{i}}}{g_{i, j_{i}}}\right),
$$

where $N_{j_{i}}$ is the background noise power user $i$ experiences (which is assumed constant) and $p_{i}(t)$ is user $i$ 's transmission power at time $t$. The sum in the above expression considers all users in the entire network. A matrix expression for all users update at time $t$ is therefore

$$
P(t)=\Gamma^{t g t}\left(\tilde{N}+\left(\tilde{Z}^{T}-E\right) P(t-1)\right),
$$

where

$$
P(t) \triangleq\left[p_{i}(t)\right], \tilde{N}=\left[\tilde{N}_{i}\right] \triangleq\left[\frac{N_{j_{i}}}{g_{i, j_{i}}}\right], \tilde{Z}=\left[\tilde{z}_{\ell k}\right] \triangleq\left[\frac{g_{\ell, j_{k}}}{g_{k, j_{k}}}\right] .
$$

The matrix $\tilde{Z}$ will, just as $\Gamma^{t g t}$, be in $\mathbb{R}^{M \times M}$. It is well known from theory of linear systems (see e.g., Kailath (1980)) that the recursion in (4.15) will converge if and only 
if all eigenvalues of $\Gamma^{t g t}\left(\tilde{Z}^{T}-E\right)$ are within the unit circle. Thus, a measure of the convergence rate is

$$
L_{c}=\bar{\lambda}\left(\Gamma^{t g t}\left(\tilde{Z}^{T}-E\right)\right) .
$$

Note that $\tilde{Z}$ and $\bar{Z}$ will have the same eigenvalues if the system is reciprocal, i.e., that the power gain is equal in uplink and downlink. In this case,

$$
L_{c}=\bar{\lambda}\left(\Gamma^{t g t}\left(\tilde{Z}^{T}-E\right)\right)=\bar{\lambda}\left(\Gamma^{t g t}\left(\bar{Z}^{T}-E\right)\right)=L_{r} .
$$

\section{Example 4.4: Multi Cell Load Estimation}

Study a system consisting of three users and two cells in which power control is implemented according to (4.15). The power gain matrix is

$$
G=\left(\begin{array}{cc}
0.53 & 0.15 \\
0.4 & 0.1 \\
0.14 & 0.8
\end{array}\right)
$$

Denote the cell represented in the first column I and the second column II. Users 1 and 2 are connected to cell I, see Figure 4.2, and the third user applies for a connection to cell II yielding a j-vector of $j=\left[\begin{array}{lll}1 & 1 & 2\end{array}\right]$. The corresponding $\bar{Z}$ and $\tilde{Z}$ matrices are thus

$$
\bar{Z}=\left(\begin{array}{ccc}
1 & 1 & 0.28 \\
1 & 1 & 0.25 \\
0.18 & 0.18 & 1
\end{array}\right) \text { and } \tilde{Z}=\left(\begin{array}{ccc}
1 & 1.33 & 0.19 \\
0.75 & 1 & 0.13 \\
0.26 & 0.35 & 1
\end{array}\right)
$$

The matrix $\bar{Z}$ has been given here simply to exemplify the calculation of it, it will not be used in the rest of the example. Note that the upper left two-by-two submatrix of $\bar{Z}$ is an all one matrix. This part of $\bar{Z}$ corresponds to a single cell system. At first, consider $\beta_{i}^{t g t}$ values according to

$$
\beta_{1}^{t g t}=0.5, \beta_{2}^{t g t}=0.3 \text { and } \beta_{3}^{t g t}=0,
$$

which corresponds to a $\Gamma$ matrix according to

$$
\Gamma^{t g t}=\operatorname{diag}\left(\begin{array}{lll}
1 & 0.43 & 0
\end{array}\right) .
$$

This yields $L_{c} \approx 0.65$ according to (4.16). Figure 4.3a shows how the users' transmission powers converge. After 100 iterations, user three is connected to cell II with $\beta_{3}^{t g t}=0.35$. Naturally this affects the transmission powers for all users in the network, as indicated in Figure 4.3a. Figure 4.3b shows what the different estimates provide in this specific example. When the new user is admitted $L_{c}$ increases up to approximately 0.70 . Thus, the fact that admitting a user in one cell requires additional transmission powers for users connected to other cells is reflected in an increased $L_{c}$. The estimate defined by (4.9) is in the first half, of course, in compliance with the load according to Definition 4.2. However, as the estimate is stationary completely insensitive to the situation in other cells, it eventually settles to the same noise rise relative load as before User 3 was admitted. The intercell-to-intracell factor was chosen to zero. In this scenario the true intercell-tointracell factor when the power control has settled is

$$
f=\frac{I^{\text {other }}}{I^{\text {own }}}=\frac{g_{3,1} p_{3}}{g_{1,1} p_{1}+g_{2,1} p_{2}} \approx 0.04 \text {. }
$$




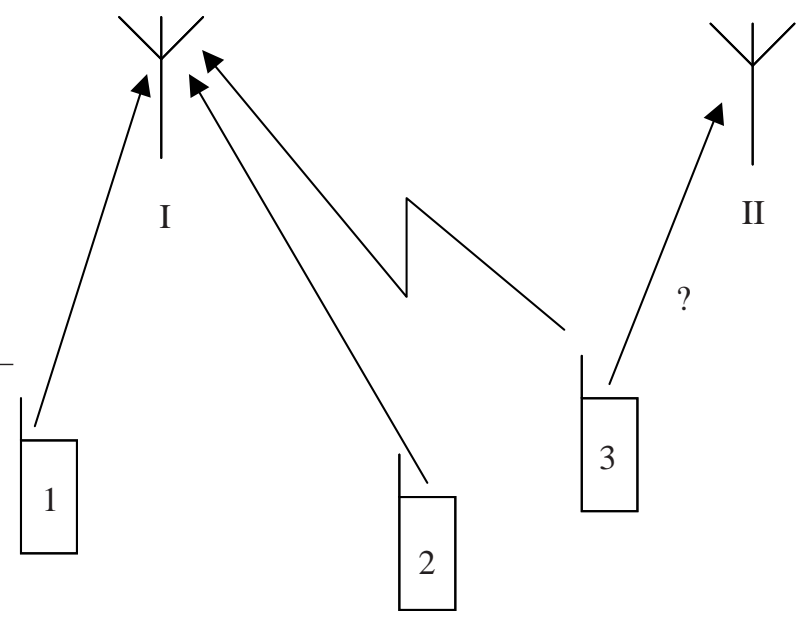

Figure 4.2: Admission of a new user. User 3 applies for a channel in cell II. Users connected to other cells will then experience increased intercell interference power.

As can be seen in the table below, $L_{r}$ equals $L_{c}$. This and other comparisons between different load definitions will be discussed in Chapter 6. Table 4.1 shows how a decentralized estimate, such as (4.9), is completely insensitive to what happens in other cells as well as to fluctuations in soft capacity. The example also indicates the trade off between cov-

Table 4.1: Four approximations of the uplink load before and after admission of user 3.

\begin{tabular}{|cc|c|c|}
\hline & & Users 1,2 & Users 1,2,3 \\
\hline$L^{n r}=1-\frac{1}{\Lambda}$ & Eq. $(4.2)$ & 0.8 & 0.84 \\
\hline$(1+f) \sum_{i} \beta_{i}$ & Eq. $(4.9)$ & 0.8 & 0.8 \\
\hline$\lambda\left(\Gamma^{t g t}(Z-E)\right)$ & Eq. $(4.12)$ & 0.65 & 0.70 \\
\hline $\bar{\lambda}\left(\Gamma^{t g t}\left(\tilde{Z}^{T}-E\right)\right)$ & Eq. $(4.16)$ & 0.65 & 0.70 \\
\hline
\end{tabular}

erage and throughput. When a new user is admitted in cell $I I$, the throughput increases but the higher transmission power of the users contributes to a decreased coverage due to limited transmission powers.

\subsubsection{Link Based Estimates}

An estimate which considers the number of links each user has can to some extent capture the soft handover gain. Assuming that maximum ratio combining is used in the combining 
a)

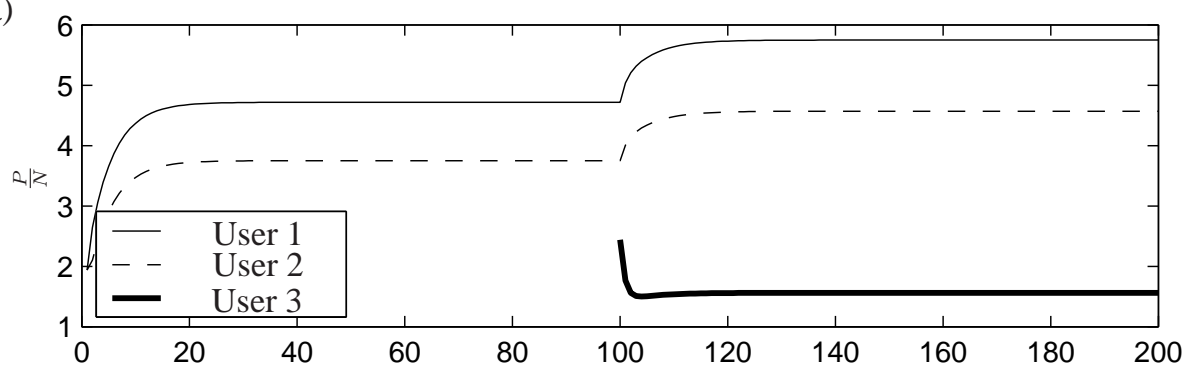

b)

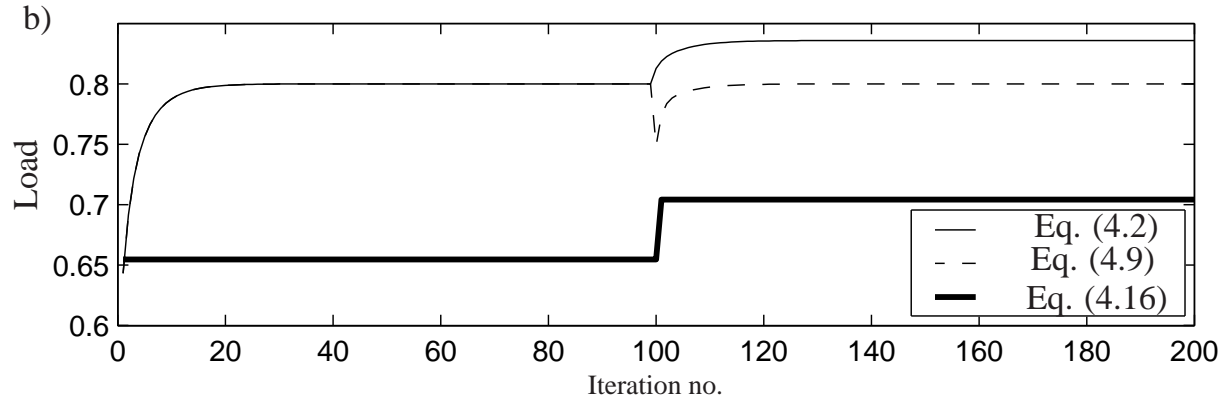

Figure 4.3: Intercell effects. a) Ratio between users' transmission power and the background noise power. b) Noise rise relative load in cell I according to Definition 4.2 and two different estimates of it. A new user is admitted in cell II at iteration number 100. The intercell-to-intracell-interference factor, $f$, is chosen to zero in (4.9).

of the locally received information, a simple way of utilizing the information regarding number of links is to assume that each user's contribution to the noise rise relative load is inversely proportional to the number of soft handover links. The noise rise relative load approximation would then be

$$
L_{j}^{n r} \approx(1+f) \sum_{i}^{M_{j}} \frac{\beta_{i}}{n_{i}},
$$

where $M_{j}$ is the number of users power controlled in cell $j$ and $n_{i}$ is user $i$ 's number of soft handover links. There has been some studies where link based admission control has been compared with interference power based admission control algorithms. For example Ishikawa and Umeda (1997) and Gunnarsson et al. (2002) show that using a link based resource compared to using an interference power based resource in the call admission control yields approximately the same performance, but the link based algorithm is far more sensitive to changes in the radio environment. Since these types of estimates indirectly consider variations in the radio environment the users experience, estimated pole capacity will change over time. 


\subsection{Discussion}

Theory regarding uplink load in cellular CDMA systems is presented in this chapter. It has been shown that, despite unlimited transmission power, the capacity of the uplink of a CDMA cellular radio system is in fact bounded by a finite time varying capacity, the pole capacity. The uplink is thus interference limited. Since the maximum capacity of the system is generally both unknown and time varying a quantity called noise rise relative load was introduced. The noise rise relative load relates the current amount of used uplink capacity to the current maximum uplink capacity, even though both are unknown. Properties of the noise rise relative load has been explored through a survey of different approaches to approximating it.

Decentralized Load This type of approximations uses information locally available in each cell. An advantage with these is of course that they can directly be used in local resource management algorithms. However, these approximations have no real knowledge of the effects that resource management decisions have on the surrounding cells. Furthermore, a decentralized approximation which makes the assumption that the other cells are equally loaded as the own cell can not fully utilize the soft capacity of a CDMA cellular system. In fact, it can be argued that some of these approximations are purely related to a hard capacity of the system. Measuring the current total interference power is one way of locally approximate how loaded the surrounding cells are. This, however, requires accurate interference power measurements - something which should not be taken for granted.

Centralized Load If a centralized approximation is used, information from several cells can be considered. The type of information may be simply the number of soft handover links each user has or, more advanced, it may be the power gains each user experiences. Some simplifying assumptions still have to be made also in the centralized cases. In the approximation where the number of links is used for example, the assumption is that the signals received in different base station are combined using maximum ratio combining, which is not true in general.

A more theoretical approach has also been handled in the chapter. A definition of downlink relative load and feasibility of the downlink power control problem was explored. By using techniques from system theory, solvability of the power control problem has been associated with a load level below one. As the power control problem is concerned with the entire network, this type of load applies to the entire network as opposed to just one base station. 


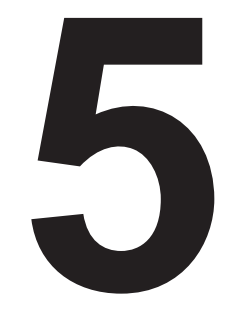

\section{Approximating Uplink Load}

This chapter contains the derivation of a number of uplink load approximations. Section 5.1 contains a derivation of a set of nonlinear equations for the uplink interference power. Different ways of approximately solving the equations lead to two fundamentally different types of approximations. These are derived in Section 5.2. Because of details in the current WCDMA standard, information required by the load approximations may not be available. Approximations explicitly handling this problem are also derived. A small comparison of the expressions is made in Section 5.3 before Section 5.4 addresses the sources of estimation errors. Finally, results of a thorough simulation study of the performance of the approximations is reported on in Section 5.7.

It is assumed that the self interference, caused by the base station not being able to utilize all of the signal received from a user, is assumed negligible in this chapter. Derivation and evaluation of the approximations can also be found in (Geijer Lundin et al., 2003c) and (Geijer Lundin et al., 2003a).

\subsection{Uplink Interference Power Expressions}

As the name suggests, noise rise relative load is closely related to the uplink noise rise. Therefore, just as the uplink noise rise, the noise rise relative load is a truly spatial quantity. A noise rise relative load expression using information gathered in several cells can be made sensitive to changes in the load caused by changed circumstances in other cells. Below, a number of approximative expressions of the noise rise relative load are derived. These expressions assume power gain values and target carrier-to-total-interference ratios to be readily available in a central node.

As a starting point for our derivation of the approximations, consider (4.1) only spe- 
cialized to base station $j$,

$$
I_{j}^{t o t}=N_{j}+\sum_{i=1}^{M} C_{i, j},
$$

where $M$ is the number of users in the entire network and $C_{i, j}$ is the power of the signal from user $i$ received in cell $j$. The signal power from user $i$ in cell $j$ is

$$
C_{i, j}=p_{i} g_{i, j}
$$

where $g_{i, j}$ is the power gain between user $i$ and base station $j$ and $p_{i}$ is the transmission power of user $i$. Combining (5.1) and (5.2) yields an expression of $I_{j}^{\text {tot }}$ in users' transmission powers. Fast power control between base stations and users is employed to counteract the influences of fast changes in the radio environment which is partially caused by the multipath fading. Due to a fast update rate and errors in the transmission power commands, the momentarily transmission powers are unknown to a central node. However, if the central node is provided with path gain measurement reports it is still possible to get an approximative expression of a low pass filtered version the users' transmission powers. The picture is somewhat complicated by the fact that a user's transmitted signal is received in several base stations. The information from these base stations can be combined in three different manners as discussed in Section 3.4. Aside from using the correct combination of the signals, it turns out that it will be useful to have approximations of it as well. The following three manners of combining the information will be used in the final noise rise relative load approximations.

Maximum Ratio Combining By approximating the combination of selection combining and maximum ratio combining with just maximum ratio combining, the total combined carrier-to-total-interference ratio is approximately the sum of the individually received carrier-to-total-interference ratios in the separate base stations

$$
\beta_{i}(t) \approx \sum_{k \in K_{i}} \frac{g_{i, k}(t) p_{i}(t)}{I_{k}^{t o t}(t)}
$$

where $K_{i}$ is the set of cells user $i$ is connected to. The approximation in the above equation is due to a nonlinear relation between CIR and CTIR, see Section 5.4.1. Solving for the transmission power above yields an approximation of user $i$ 's transmission power at time $t$,

$$
p_{i}(t) \approx \frac{\beta_{i}(t)}{\sum_{k \in K_{i}} \frac{g_{i, k}(t)}{I_{k}(t)}}
$$

Inserting this expression into (5.1) yields

$$
I_{j}^{t o t}(t)=N_{j}(t)+\sum_{i=1}^{M} p_{i}(t) g_{i, j}(t) \approx N_{j}(t)+\sum_{i=1}^{M} \beta_{i}(t) \frac{g_{i, j}(t)}{\sum_{k \in K_{i}} \frac{g_{i, k}(t)}{I_{k}^{t o t}(t)}} .
$$


Selection Combining. The above expression for the interference power in cell $j$ was derived with an assumption that softer handover is used everywhere. If soft handover is assumed instead, the signals from the different cells are combined using selection combining, i.e., the signal with the highest reliability tag is chosen. Consequently, the total combined carrier-to-total-interference ratio is

$$
\beta_{i}(t)=\max _{k \in K_{i}} \frac{g_{i, k}(t) p_{i}(t)}{I_{k}^{t o t}(t)} .
$$

Maximum Ratio and Selection Combining. Another, more accurate, assumption is to use the correct combination of soft and softer handover. Let user $i$ 's configuration of soft and softer handover be defined by $K_{i}$. The function $c\left(K_{i}, G(t), I(t)\right)$ corresponds to the denominator of (5.4) and is a combination of maximum and sums.

Divide the set of base stations user $i$ is connected to, i.e., $K_{i}$, into subsets, $K_{i, r}$ where each subset contains base stations which are all located at one specific physical site. The subsets $K_{i, r}$ are disjunct and $K_{i}=\bigcup_{r} K_{i, r}$. The function $c$ may then by defined as

$$
c\left(K_{i}, G, I\right) \approx \max _{r} \sum_{k \in K_{i, r}} \frac{g_{i, k}}{I_{k}} .
$$

Thus, maximum ratio combining is used on the signals received in each separate set $K_{i, r}$ and selection combining is then applied on the results from the maximum ratio combining. Finally, if the system is maintained at reasonable load levels, it is customary to assume that the fast power control manages to provide $\beta_{i} \approx \beta_{i}^{t g t}$.

The three different ways of approximating the transmission powers yield three different approximative expressions for the uplink interference power in cell $j$.

$$
\begin{aligned}
I_{j}^{t o t}(t) & \approx N_{j}(t)+\sum_{i=1}^{M} \beta_{i}^{t g t}(t) \frac{g_{i, j}(t)}{\sum_{k \in K_{i}} \frac{g_{i, k}(t)}{I_{k}^{t o t}(t)}} \\
I_{j}^{t o t}(t) & \approx N_{j}(t)+\sum_{i=1}^{M} \beta_{i}^{t g t}(t) \frac{g_{i, j}(t)}{\max _{k \in K_{i}} \frac{g_{i, k}(t)}{I_{k}^{t o t}(t)}} \\
I_{j}^{t o t}(t) & \approx N_{j}(t)+\sum_{i=1}^{M} \beta_{i}^{t g t}(t) \frac{g_{i, j}(t)}{c\left(K_{i}, G(t), I^{t o t}(t)\right)}
\end{aligned}
$$

Applying one of these three equations on all cells in a radio network, i.e., for $j=$ $1,2 \ldots B$, defines a system of nonlinear equations in the total received interference power.

\subsection{Uplink Load Expressions}

Three different expressions for the uplink interference power are derived in the previous section. This section is devoted to solving for the uplink noise rise using these expressions. Due to Definition 4.2 of noise rise relative load, there is a one-to-one relation 
between uplink noise rise and noise rise relative load. Thus, any of the expressions for uplink noise rise derived here can be converted to an expression for noise rise relative load.

First are some general methods for solving nonlinear equations given and then two fundamentally different ways of solving the system of nonlinear equations defined by (5.6) will be presented. In order to solve a problem of distributing required information, another set of expressions is given in a separate subsection. Finally, a discussion on how required information can be made available is included in this section.

\subsubsection{Methods for Solving Nonlinear Equations}

Each one of the equations in (5.6) defines a system of nonlinear equations. If an approximative solution to these equations can be found, it can be used to approximate the true load.

There are numerous ways of numerically solving such systems in the literature. Dennis Jr. and Schnabel (1983) give some examples. Generally the methods look for a solution to the equation $f(x, c)=0$, where $x$ is a vector of variables and $c$ are parameters of a general function $f(x, c)$.

One way of solving the equation is to linearize it or, through some other approximation, make the nonlinear equations algebraically solvable. Another way to go is through numerical algorithms. The perhaps most famous algorithm is the Newton-Raphson-method, where the approximative solution is, in each step, updated according to

$$
x^{(i+1)}=x^{(i)}-\left(\left.\frac{d f}{d x}\right|_{x=x^{(i)}}\right)^{-1} f\left(x^{(i)}\right) .
$$

A problem with this is of course that the derivative of $f(x)$ has to be available in algebraic form. If this is not the case, an approximation of the derivative can be used instead. This leads to a group of methods commonly referred to as Secant-methods.

If the nonlinear equations can be written on the form $x=f(x, c)$, fix point iterations can be applied, i.e.,

$$
x^{i+1}=f\left(x^{i}, c\right) .
$$

Note that convergence of this iteration procedure is not guaranteed in general.

Equations (5.6) are on the form given by (5.7), where $x$ would represent the total interference power, $I^{\text {tot }}$. In this work, (5.6) have been solved using linear approximations as well as fix point iterations of the nonlinear equations.

\subsubsection{Approximation I: Equal Interference Power In All Cells}

For a moment, assume that the interference powers in all cells are the same as in the cell we want to express the interference power in, i.e., $I_{k}^{t o t}=I_{j}^{t o t}$. Equation (5.6a) then becomes

$$
I_{j}^{t o t}(t) \approx N_{j}(t)+I_{j}^{t o t}(t) \sum_{i=1}^{M} \beta_{i}^{t g t}(t) \frac{g_{i, j}(t)}{\sum_{k \in K_{i}} g_{i, k}(t)} .
$$


The system of coupled nonlinear equations defined by (5.6a) is then simplified into a number of linear decoupled equations. Solving for $I_{j}^{\text {tot }}(t)$ yields an explicit expression of the interference power

$$
I_{j}^{t o t}(t)=\frac{N_{j}}{1-\sum_{i=1}^{M} \beta_{i}^{t g t}(t) \frac{g_{i, j}(t)}{\sum_{k \in K_{i}} g_{i, k}(t)}} .
$$

When comparing the above expression with (4.3), it is natural to associate the uplink relative load with

$$
L_{j}^{I M R C}(t) \triangleq \sum_{i=1}^{M} \beta_{i}^{t g t}(t) \frac{g_{i, j}(t)}{\sum_{k \in K_{i}} g_{i, k}(t)},
$$

where index $I M R C$ has been added to indicate that maximum ratio combining has been used and that the interference powers in all cells were temporarily assumed equal during the derivation. This is a practically tractable expression of the uplink relative load in cell $j$ based on readily available information. The corresponding expression for uplink noise rise is easily found by dividing (5.9) with $N_{j}$,

$$
\Lambda_{j}^{I M R C}(t) \triangleq \frac{1}{1-\sum_{i=1}^{M} \beta_{i}^{t g t}(t) \frac{g_{i, j}(t)}{\sum_{k \in K_{i}} g_{i, k}(t)}} .
$$

Starting from (5.6b) instead yields a different approximative expression for $L_{j}(t)$, namely

$$
L_{j}^{I S E L}(t) \triangleq \sum_{i=1}^{M} \beta_{i}^{t g t}(t) \frac{g_{i, j}(t)}{\max _{k \in K_{i}} g_{i, k}(t)},
$$

where the index ISEL indicates the assumption of soft handover and equal interference power in all cells. The corresponding expression of the uplink noise rise is

$$
\Lambda_{j}^{I S E L}(t) \triangleq \frac{1}{1-\sum_{i=1}^{M} \beta_{i}^{t g t}(t) \frac{g_{i, j}(t)}{\max _{k \in K_{i}} g_{i, k}(t)}} .
$$

Finally the most accurate way of combining the information used in (5.6c) results in yet another approximative expression for the uplink load

$$
L_{j}^{I B O T H}(t) \triangleq \sum_{i=1}^{M} \beta_{i}^{t g t}(t) \frac{g_{i, j}(t)}{b(K, G(t))}
$$

and for the uplink noise rise

$$
\Lambda_{j}^{N B O T H}(t) \triangleq \frac{1}{1-\sum_{i=1}^{M} \beta_{i}^{t g t}(t) \frac{g_{i, j}(t)}{b\left(K_{i}, G(t)\right)}},
$$

where $b\left(K_{i}, G\right)$ is a function combining elements of the $G$-matrix according to the configuration implied by $K_{i}$.

To summarize, the following three approximative expressions for the uplink noise rise have been derived. 


$$
\begin{aligned}
\Lambda_{j}^{I M R C}(t) & \triangleq \frac{1}{1-\sum_{i=1}^{M} \beta_{i}^{t g t}(t) \frac{g_{i, j}(t)}{\sum_{k \in K_{i}} g_{i, k}(t)}} \\
\Lambda_{j}^{I S E L}(t) & \triangleq \frac{1}{1-\sum_{i=1}^{M} \beta_{i}^{t g t}(t) \frac{g_{i, j}(t)}{\max _{k \in K_{i}} g_{i, k}(t)}} \\
\Lambda_{j}^{I B O T H}(t) & \triangleq \frac{1}{1-\sum_{i=1}^{M} \beta_{i}^{t g t}(t) \frac{g_{i, j}(t)}{b\left(K_{i}, G(t)\right)}}
\end{aligned}
$$

By using Definition 4.2, the above expressions can be rearranged into expressing the noise rise relative load instead of noise rise. The converted expressions would then equal (4.6) in a single cell system. Note that, when not utilizing soft handover, all expressions in (5.13) will be equal.

\subsubsection{Approximation II: Equal Background Noise Power}

The other approach to solving the equations defined by (5.6) is to assume equal background noise power in all cells, i.e., $N_{j}=N$. Dividing (5.6a) by $N$ yields

$$
\frac{I_{j}^{t o t}(t)}{N} \approx 1+\sum_{i=1}^{M} \beta_{i}^{t g t}(t) \frac{g_{i, j}(t)}{\sum_{k \in K_{i}} \frac{g_{i, k}(t)}{\frac{I_{k}^{t o t}(t)}{N}}}
$$

By using Definition 4.1, the ratio between total received interference power and background noise power can be substituted by the noise rise, $\Lambda_{j}$,

$$
\Lambda_{j}(t) \approx 1+\sum_{i=1}^{M} \beta_{i}^{t g t}(t) \frac{g_{i, j}(t)}{\sum_{k \in K_{i}} \frac{g_{i, k}(t)}{\Lambda_{k}(t)}} .
$$

This yields a new nonlinear system of equations where the variables are the uplink noise rise in the different base stations. The path gain values are then known parameters of the equations. This system can be solved through any of the numerical methods previously discussed in Section 5.2.1. Most simple of these is to use the previous estimate of the noise rise in base station $k$ in the right hand side of the above equation

$$
\Lambda_{j}^{N M R C}(t) \triangleq 1+\sum_{i=1}^{M} \beta_{i}^{t g t}(t) \frac{g_{i, j}(t)}{\sum_{k \in K_{i}} \frac{g_{i, k}(t)}{\Lambda_{k}^{N M R C}(t-1)}},
$$

where $N M R C$ indicates that maximum ratio combining and equal background noise in all cells were assumed during the derivation. If the users target CTIR and power gain values are constant or slowly varying, this is a fix point iteration as in (5.7). This expression of the uplink noise rise can be converted into an expression of the uplink relative load according to

$$
L_{j}^{N M R C}(t) \triangleq 1-\frac{1}{\Lambda_{j}^{N M R C}(t)} .
$$


Once again, assuming soft handover instead of softer handover provides yet another expression of the uplink noise rise,

$$
\Lambda_{j}^{N S E L}(t) \triangleq 1+\sum_{i=1}^{M} \beta_{i}^{t g t}(t) \frac{g_{i, j}(t)}{\sum_{k \in K_{i}} \frac{g_{i, k}(t)}{\Lambda_{k}^{N S E L}(t-1)}} .
$$

Also in this case, the correct combination of soft and softer handover can be used

$$
\Lambda_{j}^{N B O T H}(t) \triangleq 1+\sum_{i=1}^{M} \beta_{i}^{t g t}(t) \frac{g_{i, j}(t)}{c\left(K_{i}, g(t), \Lambda^{N B O T H}(t-1)\right)},
$$

where $c(K, g, \boldsymbol{\Lambda})$ is a function combining the noise rise vector with the $G$-matrix according to the configuration implied by the combination of soft and softer handover (here represented by $K$ ).

The following three approximations have been derived in this section.

$$
\begin{gathered}
\Lambda_{j}^{N M R C}(t) \triangleq 1+\sum_{i=1}^{M} \beta_{i}^{t g t}(t) \frac{g_{i, j}(t)}{\sum_{k \in K_{i}} \frac{g_{i, k}(t)}{\Lambda_{k}^{N M R C}(t-1)}} \\
\Lambda_{j}^{N S E L}(t) \triangleq 1+\sum_{i=1}^{M} \beta_{i}^{t g t}(t) \frac{g_{i, j}(t)}{\max _{k \in K_{i}} \frac{g_{i, k}(t)}{\Lambda_{k}^{N S E L}(t-1)}} \\
\Lambda_{j}^{N B O T H}(t) \triangleq 1+\sum_{i=1}^{M} \beta_{i}^{t g t}(t) \frac{g_{i, j}(t)}{c\left(K_{i}, g(t), \Lambda^{N B O T H}(t-1)\right)}
\end{gathered}
$$

Just as with (5.13), all of these expressions are equal when soft handover is not utilized. The expressions can be rearranged into expressing the noise rise relative load instead. In a single cell system, the corresponding expression for noise rise relative load once again coincides with (4.6). In (5.16), one iteration at each time update is used. This can be generalized into using several iterations before each update as in Algorithm 5.1. The assumption of slowly varying target CTIR and power gain values can then be relaxed. Denote by $\beta^{t g t}$ a vector containing the users' target carrier-to-total-interference ratios and by the integer $N^{i t e r}$ the number of fix point iterations performed before each update. By

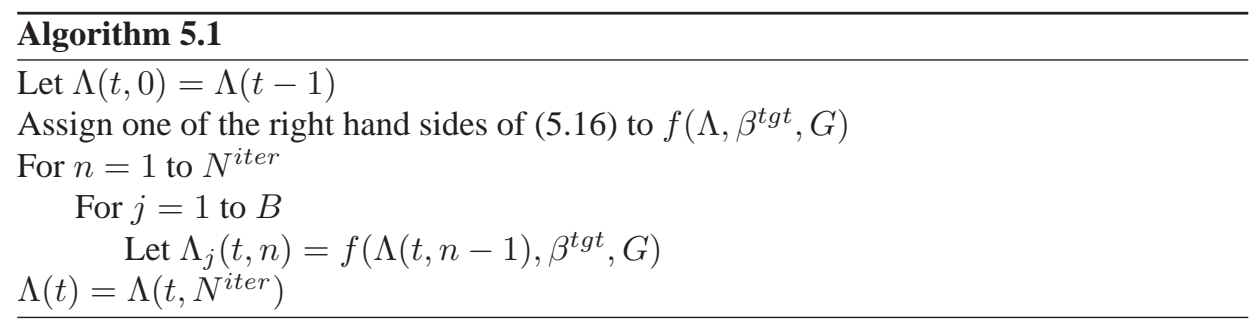

using fix point iterations like these, the expressions catch frequent updates in path gain measurements better. Convergence of this algorithm when using (5.16a) is analyzed in Chapter 6. 


\subsubsection{Approximation III: Distributed Information}

A complication concerning the distribution of required information is that the base stations may deliver the path gain measurement reports to different central nodes. For example according to the 3GPP standard, a user initiating its session in an area covered by a central, call it $R N C_{2}$, and during the session moves to an area supported by another RNC, $R N C_{1}$, will not report path gain measurements to $R N C_{1}$ but to $R N C_{2}$. A user located inside $R N C_{1}$ 's service area may thus introduce considerable interference power to the base stations without delivering any path gain reports to $R N C_{1}$. All the above expressions for uplink noise rise can only consider users reporting path gain measurements to the central the estimate resides in. Besides the obvious solution to neglect users not reporting their path gain, a set of noise rise approximations which are a combination of the techniques used in the two previous sections is proposed. Below is a derivation of the alternative expression which approximates the noise rise in base stations belonging to $R N C_{1}$.

Split the sum over users in the (5.6) into two sums, one being over the users reporting their path gain measurements to $R N C_{1}$ and the other over users reporting to other central nodes (here represented by $R N C_{2}$ ). Exemplifying with (5.6a)

$$
\begin{aligned}
I_{j}^{t o t}(t)=N_{j} & +\sum_{i \in R N C_{1}} \beta_{i}^{t g t}(t) \frac{g_{i, j}(t)}{\sum_{k \in K_{i}} \frac{g_{i, k}(t)}{I_{k}^{t o t}(t)}} \\
& +\sum_{i \in R N C_{2}} \beta_{i}^{t g t}(t) \frac{g_{i, j}(t)}{\sum_{k \in K_{i}} \frac{g_{i, k}(t)}{I_{k}^{t o t}(t)}}
\end{aligned}
$$

Here cell $j$ belongs to $R N C_{1}$. Consider just the sum over users in $R N C_{2}$ and approximate $I_{k}^{\text {tot }}(t)$ in (5.17) with the total received interference power in base station $j$. This allows us to approximate the sum as

$$
\sum_{i \in R N C_{2}} \beta_{i}^{t g t}(t) \frac{g_{i, j}(t)}{\sum_{k \in K_{i}} \frac{g_{i, k}(t)}{I_{k}^{t o t}(t)}} \approx I_{j}^{t o t}(t) \sum_{i \in R N C_{2}} \beta_{i}^{t g t}(t) \frac{g_{i, j}(t)}{\sum_{k \in K_{i}} g_{i, k}(t)}
$$

Substituting the second sum in (5.17) by the above expression and solve for $I_{j}^{\text {tot }}(t)$ yield

$$
I_{j}^{t o t}(t)=\frac{N_{j}+\sum_{i \in R N C_{1}} \beta_{i}^{t g t}(t) \frac{g_{i, j}(t)}{\sum_{k \in K_{i}} \frac{g_{i, k}(t)}{I_{k}^{t o t}(t)}}}{1-\sum_{i \in R N C_{2}} \beta_{i}^{t g t}(t) \frac{g_{i, j}(t)}{\sum_{k \in K_{i}} g_{i, k}(t)}} .
$$

Assume that $N_{j}=N_{k}=N$ and that $\Lambda_{k}(t)=\Lambda_{k}(t-1)$. Dividing the above equation by $N$ then results in an expression of the uplink noise rise which is a combination of (5.13a) and (5.16a)

$$
\Lambda_{j}^{R N C M R C}(t) \triangleq \frac{1+\sum_{i \in R N C_{1}} \beta_{i}^{t g t}(t) \frac{g_{i, j}(t)}{\sum_{k \in K_{i}} \frac{g_{i, k}(t)}{\Lambda_{k}^{R N C M R C}(t-1)}}}{1-\sum_{i \in R N C_{2}} \beta_{i}^{t g t}(t) \frac{g_{i, j}(t)}{\sum_{k \in K_{i}} g_{i, k(t)}}} .
$$


Note that the sum in the denominator represents the noise rise relative load that users reporting to $R N C_{2}$ introduce to cell $j$. Neglecting these users results in a larger denominator, which yields a smaller approximation.

This approximation is interesting since $R N C_{2}$ can now send a message to $R N C_{1}$ containing the additional noise rise relative load that users belonging to $R N C_{2}$ introduce in each cell controlled by $R N C_{1}$. This message would then be far smaller than one containing the complete path gain information regarding these users. Obviously soft handover can be assumed to be used everywhere here as well, or the actual combination of soft and softer handover can be assumed. This gives three types of expressions using the technique with sending relative load information between different RNCs.

$$
\begin{gathered}
\Lambda_{j}^{R N C M R C}(t) \triangleq \frac{1+\sum_{i \in R N C_{1}} \beta_{i}^{t g t}(t) \frac{g_{i, j}(t)}{\sum_{k \in K_{i}} \frac{g_{i, k}(t)}{\Lambda_{k}^{R N C M R C}(t-1)}}}{1-\sum_{i \in R N C_{2}} \beta_{i}^{t g t}(t) \frac{g_{i, j}(t)}{\sum_{k \in K_{i}} g_{i, k(t)}}} \\
\Lambda_{j}^{R N C S E L}(t) \triangleq \frac{1+\sum_{i \in R N C_{1}} \beta_{i}^{t g t}(t) \frac{g_{i, j}(t)}{\max _{k \in K_{i}} \frac{g_{i, k}(t)}{\Lambda_{k}^{R N C S E L}(t-1)}}}{1-\sum_{i \in R N C_{2}} \beta_{i}^{t g t}(t) \frac{g_{i, j}(t)}{\max _{k \in K_{i}} g_{i, k(t)}}} \\
\Lambda_{j}^{R N C B O T H}(t) \triangleq \frac{1+\sum_{i \in R N C_{1}} \beta_{i}^{t g t}(t) \frac{g_{i, j}(t)}{c\left(K_{i}, G(t), \Lambda^{R N C B O T H}(t-1)\right.}}{1-\sum_{i \in R N C_{2}} \beta_{i}^{t g t}(t) \frac{g_{i, j}(t)}{b\left(K_{i}, g_{i, k(t)}\right)}}
\end{gathered}
$$

\subsubsection{Required Information}

All the above derived expressions for the uplink load rely on knowledge of users' target carrier-to-total-interference-ratio, $\beta^{\text {tgt }}$, which are assumed known by the system. They also need path gain measurements, which can be made available in two different ways: (3GPP, 2000a, 1999)

M1: The mobile stations are requested to periodically (but not necessarily synchronously) report pilot power measurements. As an example, pilot power from the six strongest base stations can be reported at a rate of $0.5 \mathrm{~Hz}$.

M2: For handover purposes, the mobile typically reports similar measurements in an event-driven fashion. It measures the pilot powers from the neighboring cells and reports up to the six strongest path gains at handover events.

In both cases, the channel is assumed reciprocal, i.e., the uplink path gain is assumed approximately equal to the corresponding downlink path gain with respect to distance dependent path loss and shadow fading. Multi path fading is assumed filtered out in the 
lower layer filtering. Furthermore, these different strategies only provide data from a limited set of mobile-to-base path gains. The remaining path gains, however, are considered small and set equal to zero. According to the 3GPP standard the individual measurement errors in relative path gain should not be greater than $1.5 \mathrm{~dB}$ with a probability of $90 \%$ (3GPP, 2000a).

\subsection{Comparison of the Uplink Load Expressions}

In the previous section two fundamentally different ways of solving the nonlinear system of equations implied by (5.6a) are presented. The approximate expressions derived in Section 5.2.2 which assume the interference power to be equal in all cells, may at first seem redundant since other expressions which are derived under far more reasonable assumptions are available, the ones assuming equal background noise. The approximations $\Lambda^{I M R C}, \Lambda^{I S E L}$ and $\Lambda^{I B O T H}$ have, however, one major advantage compared to the iterations. When trying to approximate the uplink noise rise in base station $j$, the interference power in base station $k$ was approximated with that in base station $j$, i.e., $I_{k}^{\text {tot }}=I_{j}^{\text {tot }}$. In the case base station $j$ is the base station with the highest interference power in the area, this implies, starting with (5.6a),

$$
\begin{aligned}
I_{j}^{t o t} & \approx N_{j}+\sum_{i=1}^{M} \beta_{i}^{t g t} \frac{g_{i, j}}{\sum_{k \in K_{i}} \frac{g_{i, k}}{I_{k}^{t o t}}} \\
& \leq N_{j}+\sum_{i=1}^{M} \beta_{i}^{t g t} \frac{g_{i, j}}{\sum_{k \in K_{i}} \frac{g_{i, k}}{I_{j}^{t o t}}}=N_{j}+I_{j}^{t o t} \sum_{i=1}^{M} \beta_{i}^{t g t} \frac{g_{i, j}}{\sum_{k \in K_{i}} g_{i, k}}=L_{j}^{I M R C} .
\end{aligned}
$$

This property of the estimate can be useful when using the estimate in RRM algorithms. Since the approximation is likely to be higher then the true load, the RRM algorithms will receive an earlier warning of a potential future congestion.

The main strength of the above expressions for uplink load is that they, like the true interference power, depend on the actual situation in several cells and do not rely on an intercell-to-intracell-interference factor, $f$. Furthermore, since the expressions involve the users' carrier-to-total-interference target, users who require high transmission power will inherently be given less coverage if an expression like this is used in the resource management algorithms. To exemplify, study (5.10)

$$
L_{j}^{I M R C}(t)=\sum_{i=1}^{M} \beta_{i}^{t g t}(t) \frac{g_{i, j}(t)}{\sum_{k \in K_{i}} g_{i, k}(t)} .
$$

Each users' contribution is a product of the target carrier-to-total-interference ratio and a ratio between path gains. A user far from the own base station but close to another, will have a small gain to its own base station $\left(g_{i, k}\right)$ and a relatively large gain to the other base station $\left(g_{i, j}\right)$. Therefore, the ratio of the path gains will be relatively large which can be compensated for with a small target carrier-to-total-interference ratio. This one of the ideas used for designing radio resource allocation algorithms in Chapter 7. 


\subsection{Sources of Estimation Errors}

A number of different effects and approximations degrade the performance of the load approximations. These include:

- The nonlinear relation between CIR and CTIR (see Section 5.4.1)

- TX increase (see Section 5.4.2)

- The soft(er) handover assumption

- Imperfect power control

- The assumption regarding relation between the uplink interference power, alternatively the background noise power, in different base stations

- The assumption that unknown path gains are equal to zero

- Path gain measurement errors according to 3GPP (2000a) and sparsely sampled path gain measurement reports

These error sources' affect on the approximation accuracy is investigated through simulations in Section 5.7.

\subsubsection{Nonlinear Relation Between CIR and CTIR}

When deriving (5.3) it is assumed that the sum over the received CTIR in different base stations $k, \beta_{i, k}$, equals the $\beta_{i}$ which is required by the user's service, i.e.,

$$
\beta_{i}=\sum_{k \in K_{i}} \beta_{i, k}
$$

This is not true in general where there may be more than one term in the sum, even if $\sum_{k \in K_{i}} \gamma_{i, k}=\gamma_{i}$. A simple proof is

$$
\sum_{k \in K_{i}} \beta_{i, k}=\sum_{k=1}^{3} \frac{\gamma_{i, k}}{1+\gamma_{i, k}} \geq \sum_{k=1}^{3} \frac{\gamma_{i, k}}{1+\gamma_{i}}=\frac{\gamma_{i}}{1+\gamma_{i}}=\beta_{i}
$$

where equality holds if and only if two of the $\gamma_{i, k}$ :s are zero (which corresponds to no soft handover). Thus, the sum over the separately received CTIR is larger than the experienced CTIR. Therefore, this contributes to under estimating the users' transmission powers.

How big the difference is depends on the relation between the separately received CIR values; if a user is almost solely connected to one base station, the difference is small. The combined CIR also influences how big the difference is. The bigger the combined CIR is, the bigger the difference may be. 


\subsubsection{TX Increase}

For simplicity, assume that all users in the system is power controlled form exactly one base station. TX increase is a result of fast power control adjusting each user's transmission power to compensate for multi path fading dips in the power gain between mobile and connected base station. The mobile uses a high power during deep fades. This may yield a considerable interference increase to neighboring cells since a dip in the power gain to the connected base station does not always imply an, as deep, dip to the neighboring base stations.

To exemplify, consider a scenario where mobile one is connected to base station two. We are interested in its interference power contribution to base station three. Due to power control, each user's transmission power is approximately inversely proportional to the momentary power gain between the user and the controlling base station, i.e.,

$$
p_{1} \propto \frac{1}{g_{1,2}}
$$

Base station three will receive the signal $p_{1} g_{1,3}$ which is proportional to the ratio between the momentarily power gains, i.e.,

$$
C_{1,3} \propto \frac{g_{1,3}}{g_{1,2}}
$$

The path gain reports provided by the users do not include multi path fading (the actual reported value is low pass filtered; $F\left\{g_{i, j}\right\}$ ). Since the uplink load approximations, unlike fast power control, use users' path gain reports there will be a part of the uplink interference power that will not be counted for in the approximations. Essentially, it is possible to observe $\frac{F\left\{g_{1,3}\right\}}{F\left\{g_{1,2}\right\}}$ but not $\frac{g_{1,3}}{g_{1,2}}$, which is related to the actual interference power contribution in base station three. In Figure 5.1a, the ratio of low-pass filtered versions of the path gain measurements (solid) is plotted. Of course this will not detect the high peaks in the ratio of the momentarily power gains (dashed). Perhaps momentarily sharp peaks are tolerable by base station three. A better model could therefore be to consider the low-pass filtered load contribution which is essentially proportional to a low pass filtered version of the ratio between the power gain to base station three and base station one, $F\left\{\frac{g_{1,3}}{g_{1,2}}\right\}$. However, as indicated by Figure 5.1b, the load contribution is still underestimated. Essentially, this exemplifies Jensen's inequality (Billingsley, 1995)

$$
f(\mathrm{E}(x)) \leq \mathrm{E}(f(x))
$$

where $f(x)$ is a convex function and $\mathrm{E}(x)$ denotes the expected mean with respect to $x$. The solid line in the Figure 5.1b thus represents the quantity used in our expressions of the average noise rise while the dashed line represents the quantity that corresponds to user $i$ 's actual contribution to the average uplink noise rise in base station $j$. This additional contribution in inter-cell-interference is referred to as $T X$ increase, defined as follows.

Definition 5.1 (TX increase). The $T X$ increase, $\tau$, of a connection is the ratio between expected transmission power with multipath fading and the expected transmission power without multipath fading. 
a)

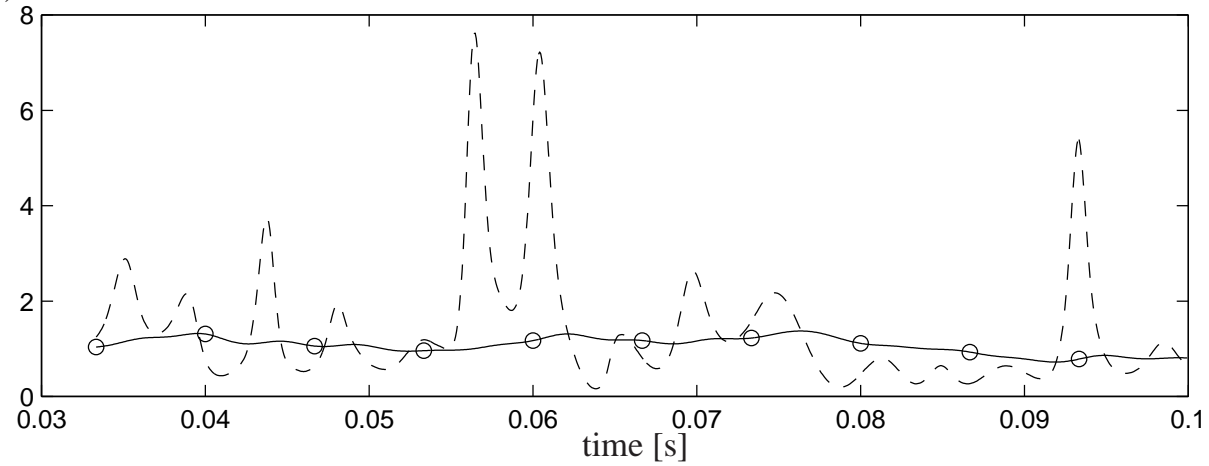

b)

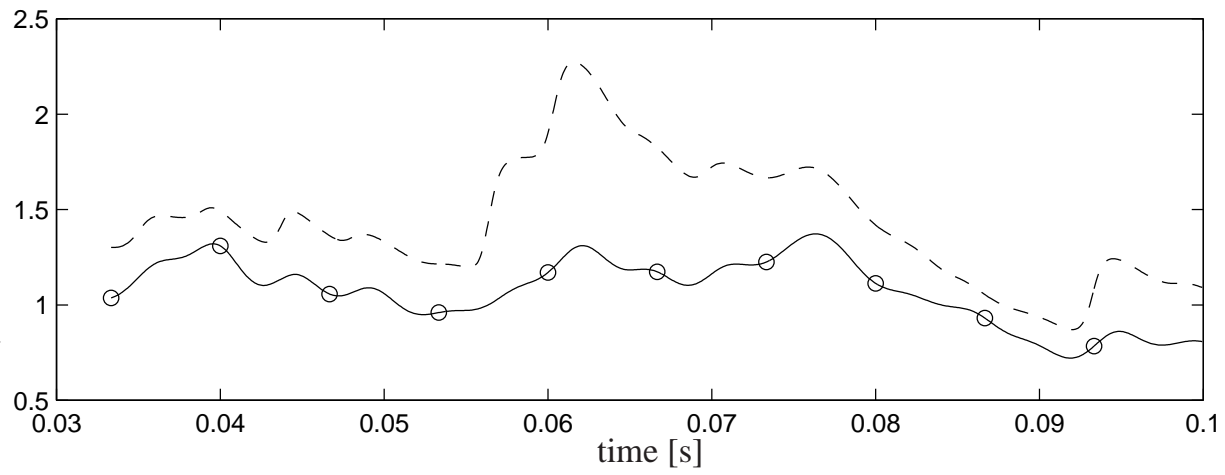

Figure 5.1: a) Ratio between filtered power gain measurements $\frac{F\left\{g_{i, j}\right\}}{F\left\{g_{i, k}\right\}}$ (solid) and momentary measurements $\frac{g_{1,3}}{g_{1,2}}$ (dashed). The momentary high peaks in the ratio between the momentary measurements are not present in the ratio between the filtered versions. b) Ratio between filtered measurements (solid) and filtered ratio, $F\left\{\frac{g_{1,3}}{g_{1,2}}\right\}$ (dashed). The average of the filtered ratio between momentary measurements (dashed) is higher than the ratio between the filtered measurements (solid).

Since the uplink load expressions, unlike fast power control, use power gain reports from users, the approximations do not include the part of the uplink load caused by TX increase.

Aside from the approximations, there are ideas of how to schedule transmission in such a way that transmission during deep fades are avoided. This will make the variance of the momentary power gain values less and hence decrease the TX increase effect. An example of such a work is Törnqvist et al. (2004). TX increase has been studied by e.g., Ariyavisitakul and Chang (1993); Hashem and Sousa (1999) and Sipilä et al. (1999).

\subsection{TX Increase Compensation}

Boujemaa et al. (2002); Sipilä et al. (1999) provide expressions for the average trans- 
mission power increase when utilizing the diversity gain of a rake receiver. Sipilä et al. (1999) also concludes that the expressions, which are derived under an assumption of perfect power control, are reasonable even with imperfect power control. The amount of additional average intercell interference power induced by TX increase is a function of how many multi path fading rays the receiver considers and the relative strength these have. A first result is that in case there are $N$ equally strong rays the interference power is increased a factor $N /(N-1)$. We study a more general and practically interesting case, where the rays have different strength. If the strength of each received multi path ray, $X_{k}, k=1,2 \ldots, N$ can be assumed exponentially distributed, the weighted sum, $\sum a_{k} X_{k}$, will have a probability density function which can be approximated by

$$
\sum_{k=1}^{N} \frac{\pi_{k}}{a_{k}} e^{-\frac{x}{a_{k}}}, x \geq 0
$$

where $\pi_{k}$ is

$$
\pi_{k}=\prod_{i=1, i \neq k}^{N} \frac{a_{k}}{a_{k}-a_{i}} .
$$

This is an approximative expression because perfect utilization of each received multi path ray was assumed during the derivation. Let the coefficients $a_{k}$ be normalized so that their sum equals 1 . The expected average transmission power increase can now be expressed as

$$
\tau=\sum_{k=1}^{N} \frac{a_{k}^{N-2} \log \left(a_{k}\right)}{\prod_{i=1, i \neq k}^{N} a_{k}-a_{i}}, \sum_{k=1}^{N} a_{k}=1,
$$

where $\tau$ is the ratio between expected transmission power with multi path fading and the transmission power without. Note, that the above expression requires all the relative strengths, $a_{k}$, to be unique. In a practical scenario, however, this is always the case.

Given the characteristics of the radio channel, and assuming that these characteristics are the same in all surrounding cells, it is thus possible to calculate a factor $\tau_{i, j}$ which approximates the additional received carrier power from user $i$ in base station $j$. The factors can then be used by the noise rise approximations in (5.13) and (5.16). Accordingly, Algorithm 5.1 can be adjusted to incorporate this TX increase compensation. This idea is used in Algorithm 5.2 which is applicable to a system not using soft handover. The algorithm is only exemplified with $\Lambda^{N M R C}$, of course any other of the recursions derived earlier can be applied. This assumes all $a_{k}$ to be unique. The corresponding algorithm

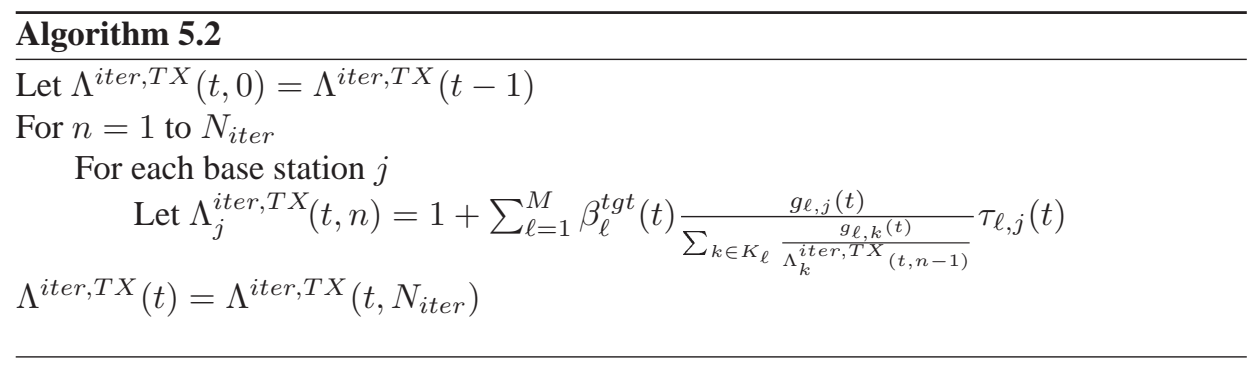


applicable to a system using soft handover requires more detailed consideration of each mobile's set $K_{\ell}$. TX increase compensation can also be applied to $L^{l i n}$,

$$
L_{j}^{l i n, T X}(t) \triangleq \sum_{\ell=1}^{M} \beta_{\ell}^{t g t}(t) \frac{g_{\ell, j}(t)}{\sum_{k \in K_{\ell}} g_{\ell, k}(t)} \tau_{\ell, j}(t) .
$$

\subsection{Summary of Proposed Approximations and Com- pensation Methods}

In the previous sections a number of uplink load approximations have been proposed. This section will try to bring some clarity into how these approximations relate and when to use which. Basically there are two dimensions, one being what kind of signal combining is assumed in the receiver and the other dimension being how a system of nonlinear equations is solved.

Three different ways of combining the signals in the base station and radio network controller are studied. These are maximum ratio combing or selection combining and, perhaps the most obvious, using the correct combination of maximum ratio combining and selection combining. All of these three choices on type of combining leads to different system of nonlinear equations that needs to be solved, see (5.6). The second dimension is about how these equations are solved. The three alternatives that were proposed are relaxing the interconnections between the equations, using fixed point iterations or using a combination of the two. These are found in Section 5.2.2, Section 5.2.3 and Section 5.2.4, respectively. The last method is designed to solve a problem with distributing path gain information between different central nodes, or radio network controller in the case of WCDMA, of a system. This is especially useful in networks where base stations in a geographically limited area do not necessarily belong to the same central node.

Table 5.1 summarizes the nine approximations given by the two dimensions. TX in-

Table 5.1: Summary of load approximations.

\begin{tabular}{|c|c|c|c|}
\hline & $I_{k}=I_{j}$ & $N_{k}=N_{j}=N$ & $I_{k}=I_{j}$ and $N_{k}=N_{j}=N$ \\
\hline MRC & $\Lambda^{\text {IMRC }}$ & $\Lambda^{\text {NMRC }}$ & $\Lambda^{\text {RNCMRC }}$ \\
\hline SEL & $\Lambda^{\text {ISEL }}$ & $\Lambda^{\text {NSEL }}$ & $\Lambda^{\text {RNCSEL }}$ \\
\hline MRC and SEL & $\Lambda^{\text {IBOTH }}$ & $\Lambda^{\text {NBOTH }}$ & $\Lambda^{\text {RNCBOTH }}$ \\
\hline
\end{tabular}

crease is a term for the additional intercell interference that power control in combination with multi path fading causes. This contribution to the total received interference power can not be detected using path gain measurements only. Section 5.5 introduces a method for approximating this increase in received interference power. The technique is applicable to all of the approximations in Table 5.1. 


\subsection{Simulations}

Performance of the load approximations derived in this chapter is in this section evaluated using simulations. The simulator models many of the characteristics of a true system. See Appendix A.1 for more details of the simulator. The performance of the approximations in terms of mean and standard deviation of single errors will be evaluated for different load levels. Reasons for the approximation errors will also be discussed along the way.

\subsubsection{Simulation Setup}

The simulator used when evaluating the performance of the approximations is discussed in Appendix A.1. The simulation area is a system of 21 base stations located at 7 different sites. In order to avoid border effects in the outermost cells, a wrapping technique has been used. The wrapping applies to both interference and user movement.

For the purpose of evaluating the performance of the expressions for noise rise derived in Section 5.2, momentary true uplink noise rise and all the approximative expressions for it is sampled every frame, i.e., 100 times a second. The true noise rise is calculated using Definition 4.1, in which the interference power is found using (4.1).

Measurements of path gains are subject to errors according to (3GPP, 2000a), i.e. a $90 \%$ confidence interval of $1.5 \mathrm{~dB}$. The users' inability to report path gain to all base stations is modeled through limiting each report to the six strongest base stations. This is also the maximum number of cells that is reported in a WCDMA system.

The total power gain is calculated using two factors; $g^{s}$ and $g^{m p}$. Antenna gain, distance attenuation and shadow fading are all represented by $g^{s}$ while $g^{m p}$ represents the variations caused by the multi path fading. The true noise rise is thus calculated according to

$$
\Lambda_{j}(t)=\frac{N+\sum_{i}^{M} g_{i, j}^{s}(t) g_{i, j}^{m p}(t) p_{i}(t)}{N},
$$

where $N$ is a constant representing the thermal noise only.

Each approximative expression in Section 5.2 is then compared to the true noise rise, time instant by time instant. In the following sections of this chapter, statistics of the resulting differences in noise rise are reported for different true noise rise levels.

Two different bit rates are used in the simulations, $64 \mathrm{kbps}$ and $192 \mathrm{kbps}$. The users have no upper transmission power limit in order to isolate the sources of estimation errors considered here.

\subsubsection{Measurement Report Frequency}

The approximative expressions in Section 5.2 rely on measurements of the path gains between user and base station. In reality, complete knowledge of the entire path gain matrix is not available since path gain reports are sparsely spread in time and they do not cover all base stations. Therefore this section is devoted to studying the expressions' sensitivity to limited path gain knowledge. As a comparison, Figure 5.2 shows average error of $\Lambda^{N B O T H}$ and $\Lambda^{I B O T H}$ (which both can be found in (5.16)) when knowing the whole uplink path gain matrix and actual received $\beta_{i}$. Already under these relatively idealistic circumstances, an effect of approximating the interference power in all cells 
with the interference power in the own cell is apparent; the average error when using $\Lambda^{I B O T H}$ deviates from the trend for high loads. The samples representing this part of the curve have probably been sampled in the cells which experience the highest load. Thus, assuming all other cells to have the same high noise rise contributes to an over estimation, which makes the average error smaller since it is an underestimation. The path gain

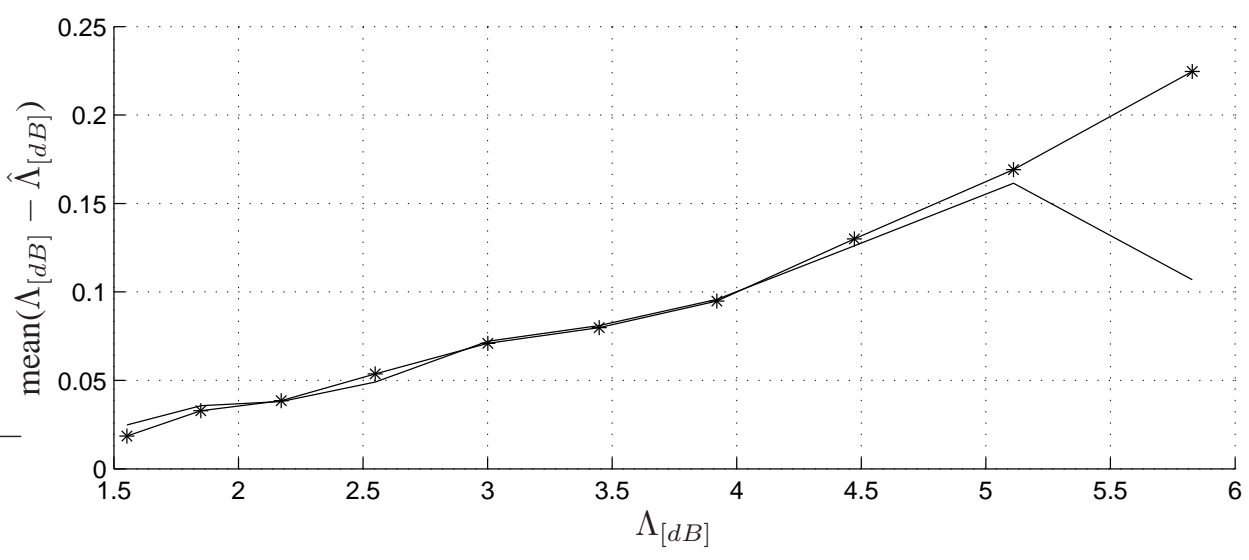

Figure 5.2: Mean error in noise rise when knowing the entire path gain matrix. '*, represents $\Lambda^{N B O T H}$ and '-' represents $\Lambda^{I B O T H}$

measurements which all the expressions evaluated herein depend on can be made available in many different ways. Two different ways, which are both in the current 3GPP standard, are described in Section 5.2.5. Basically the one referred to as M1 schedules the path gain reports in a periodic manner with a periodicity of $0.5 \mathrm{~Hz}$, while when using M2 users report only in conjunction with soft hand over requests, i.e., in an event driven manner. In this section, the expressions sensitivity to how the measurements are scheduled will be investigated. Not surprisingly, as can be seen in Figure 5.3, using event driven path gain reports (i.e., M2) results in far less reports per user and hence less signaling overhead for the system. More interesting is the fact that using M2 does not necessarily imply a worse approximation, see Figure 5.4 and 5.5 which show the average estimation error of $\Lambda^{N M R C}, \Lambda^{N S E L}$ and $\Lambda^{N B O T H}$ when using users traveling at an average speed of 10,20 and $70 \mathrm{~km} / \mathrm{h}$, respectively. A comparison between expressions assuming $I_{k}=I_{j}$ as opposed to $N_{k}=N_{j}$ shows that there is no statistical difference in average error. However, using M1 results in considerably larger standard deviation when assuming $I_{k}=$ $I_{j}$, i.e., $\Lambda^{I M R C}, \Lambda^{I S E L}$ or $\Lambda^{I B O T H}$ (all from (5.13)), see Figure 5.6 and 5.7.

Only when traveling at a rather high speed, $70 \mathrm{~km} / \mathrm{h}$, using M1 or M2 results in different error statistics for $\Lambda^{N M R C}, \Lambda^{N S E L}$ and $\Lambda^{N B O T H}$. At this speed, M1 provides a better average error but as can be seen in Figure 5.6, the standard deviation is much higher compared with using M2. The average error may be canceled by an error correction method, but we can do almost nothing to combat a high standard deviation. Average error cancellation also requires a low standard deviation, which is why M2 can be considered providing an even better approximation than M1. The significant increase in standard deviation for low load levels when using M1 is explained by the approximation being far 
from accurate in a few instants. The statistics is thus somewhat misleading in this case.

It is the users that are close to the cell border that are the most important ones to have somewhat accurate path gain knowledge of since these are the users that cause most of the inter-cell-interference. Since these users are more likely to change their soft handover setup compared to users within the cell, using M2 thus mean that it is also more likely that these users provide reports. Simulations shown in this section implies that scheduling the users' path gain reports in a event driven manner, such as M2, provides an at least equally good approximation of the uplink noise rise while requiring less signaling overhead.

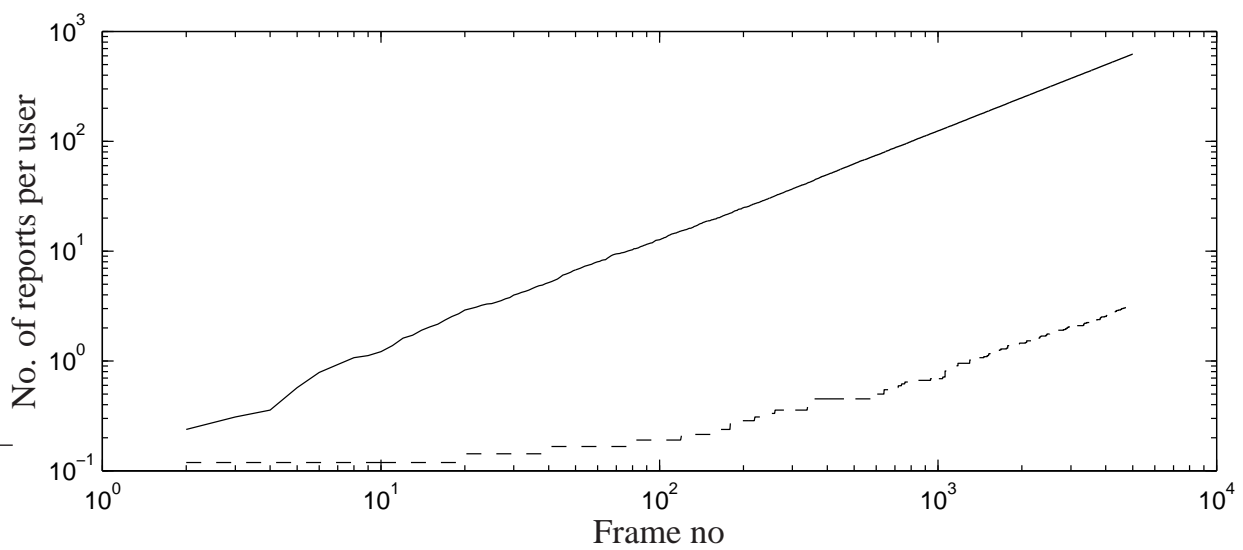

Figure 5.3: Cumulative sum over number of reports per user for M1 (solid) and M2 (dashed)

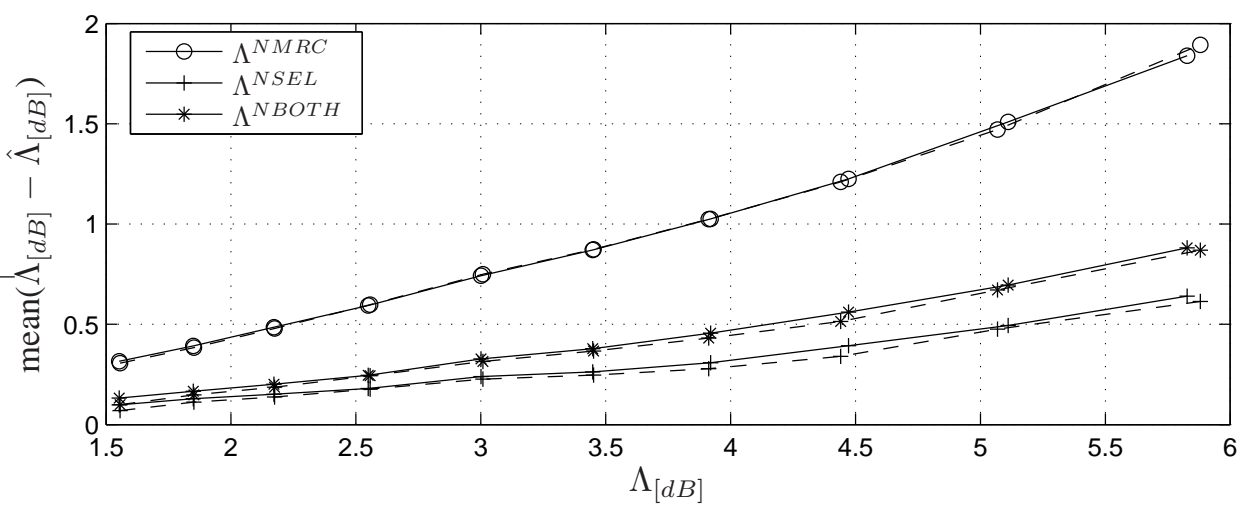

Figure 5.4: Mean error in noise rise. User speed: $10 \mathrm{~km} / \mathrm{h}$. Solid:M1, dashed:M2.

\subsubsection{One Radio Network Controller}

In the previous section it is shown that the expressions are practically independent of the type of path gain reporting, periodic or event-based, and therefore only event-based reports are considered in this section. 


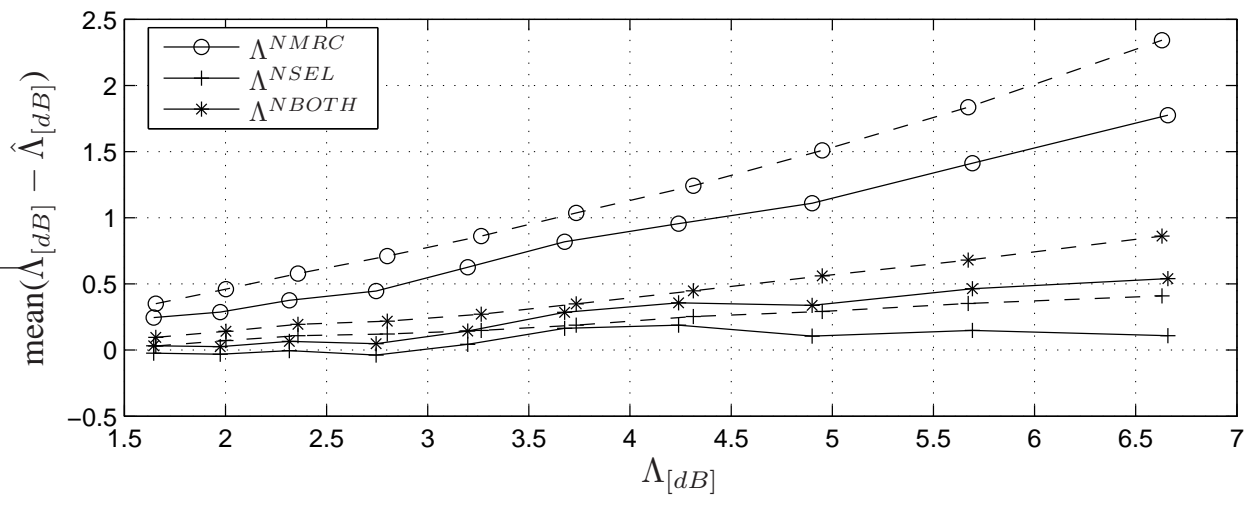

Figure 5.5: Mean error in noise rise. User speed: 70 km/h. Solid:M1, dashed:M2.

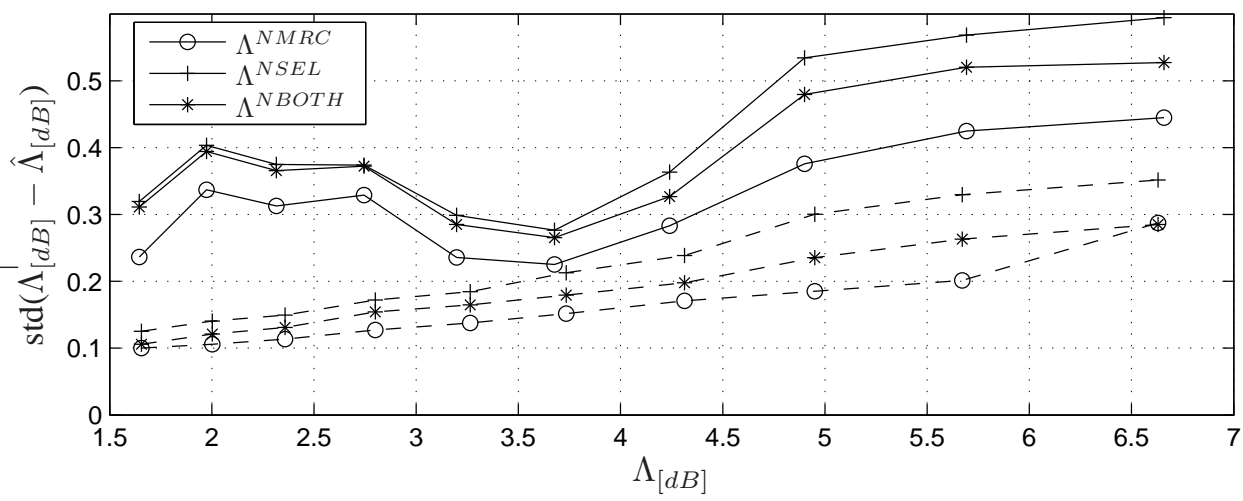

Figure 5.6: Standard deviation of single noise rise errors. All is for $70 \mathrm{~km} / \mathrm{h}$. solid:M1, dashed:M2

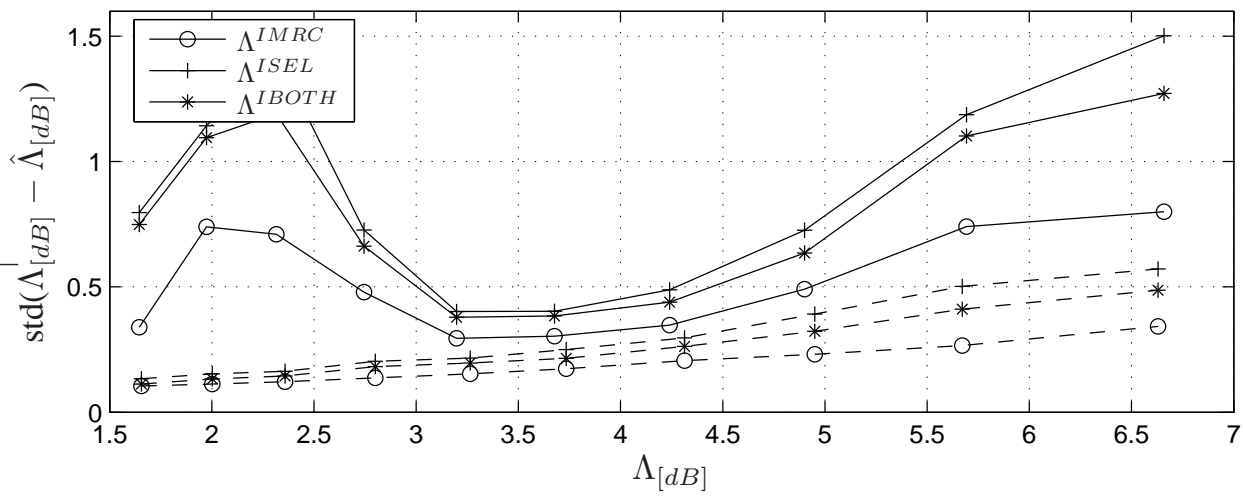

Figure 5.7: Standard deviation of single noise rise errors.All is for $70 \mathrm{~km} / \mathrm{h}$. solid:M1, dashed:M2 


\section{Uniform Traffic With Single Service}

Figure 5.8 shows the performance of four different expressions when using $64 \mathrm{kbps}$ streaming users. As each user has no more than one link, assuming soft or softer handover makes no difference in this scenario. The major gap between the lines is due to TX increase (see Section 5.4.2), since multi path fading is not simulated in the runs corresponding to the dashed lines. The conclusion from this figure is that TX increase does give a considerable contribution to the total error. Also, all four expressions produce an underestimate of the uplink noise rise, mainly due to that measurements of all path gains are not available. A part of the error due to TX increase can be eliminated by using the

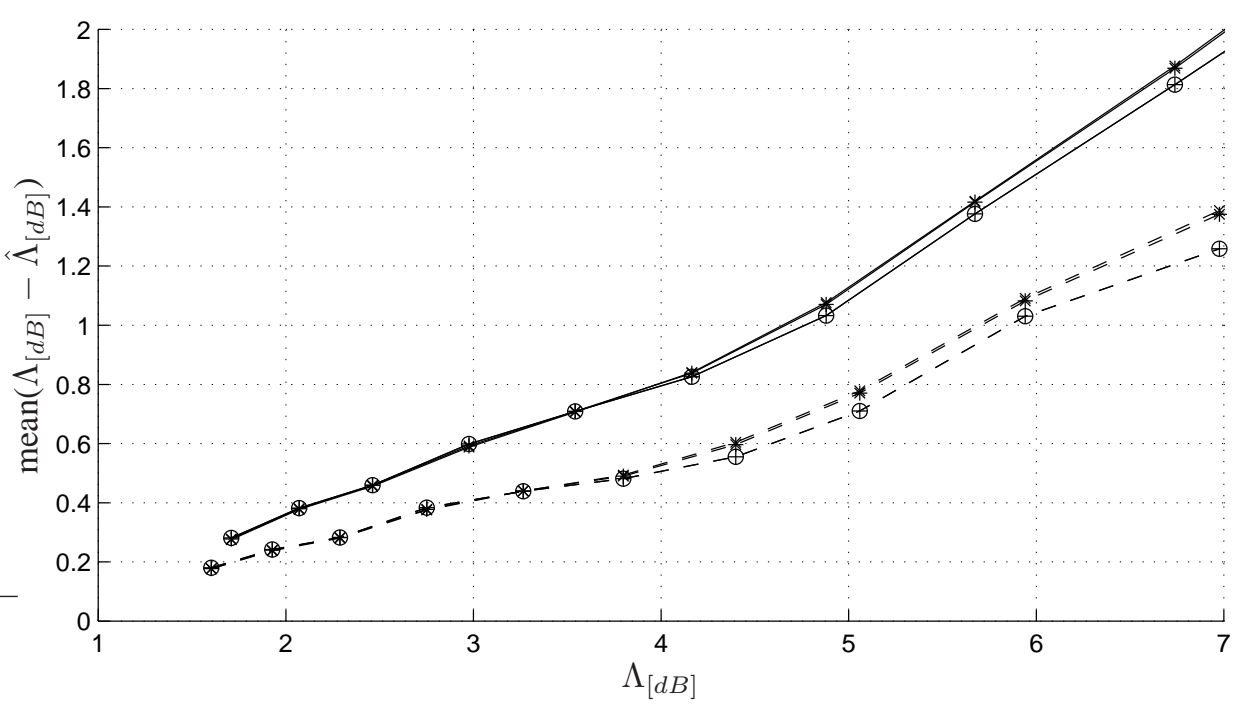

Figure 5.8: Error in noise rise with 64 kbps users. One link per user. Dashed: No multi path fading. 'o': $\Lambda^{I M R C},{ }^{\prime} X^{\prime}: \Lambda^{N M R C}$, ' + ': $\Lambda^{I S E L},{ }^{*}{ }^{\prime}: \Lambda^{N S E L} . T X$ increase clearly has an impact on the approximations.

expressions for the additional transmission power in Section 5.5. If the factors by which the separate received multi path rays are weighted with, i.e., $a_{k}$ in (5.19), are known it is possible to apply Algorithm 5.2. Figure 5.9 shows that it is then possible to eliminate most of the error in average. In Figure 5.10 a more realistic scenario is shown where each user is allowed up to three handover links at a time. First of all, assuming softer handover $\left(\Lambda^{I M R C}\right.$ and $\left.\Lambda^{N M R C}\right)$ gives larger average error compared to assuming soft handover (i.e., $\Lambda^{I S E L}$ and $\Lambda^{N S E L}$ ) or both soft and softer handover (i.e., $\Lambda^{I B O T H}$ and $\left.\Lambda^{N B O T H}\right)$. This is natural since assuming softer handover everywhere means overestimating the system's ability to correctly receive signals, which in turn means underestimating the required transmission powers. Under these circumstances, i.e., almost equal load in all cells, the errors from all six expressions are small in average and has fairly low standard deviation. Using any of the approximations in (5.16) provides equal average error as those in (5.13), but with lower standard deviation. This is explained by the fact that assuming equal background noise power makes the performance of the expression 


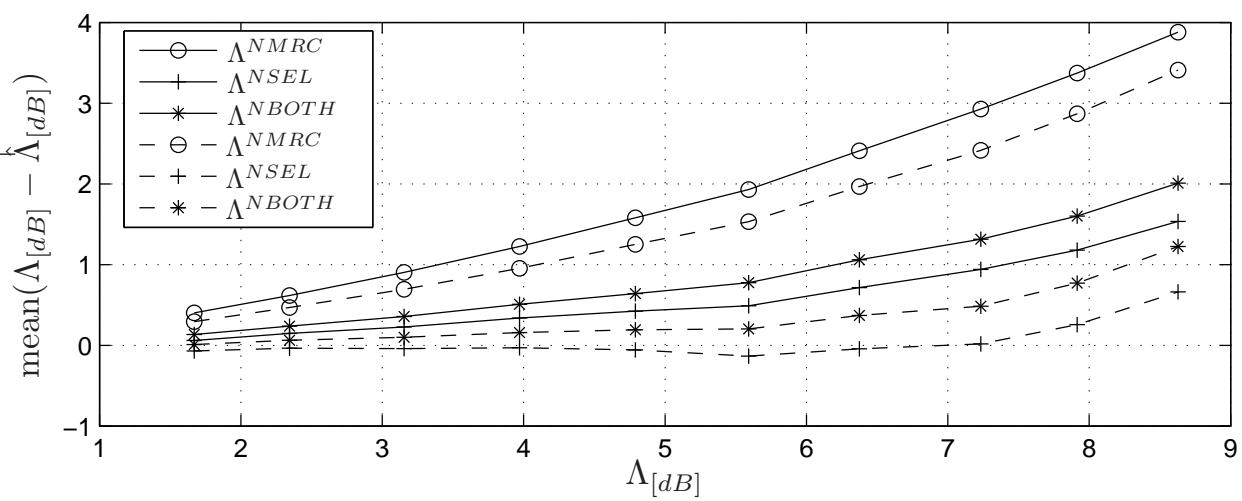

Figure 5.9: Error in noise rise with $64 \mathrm{kbps}$ users. TX increase compensation has be applied for the dashed lines.

a)
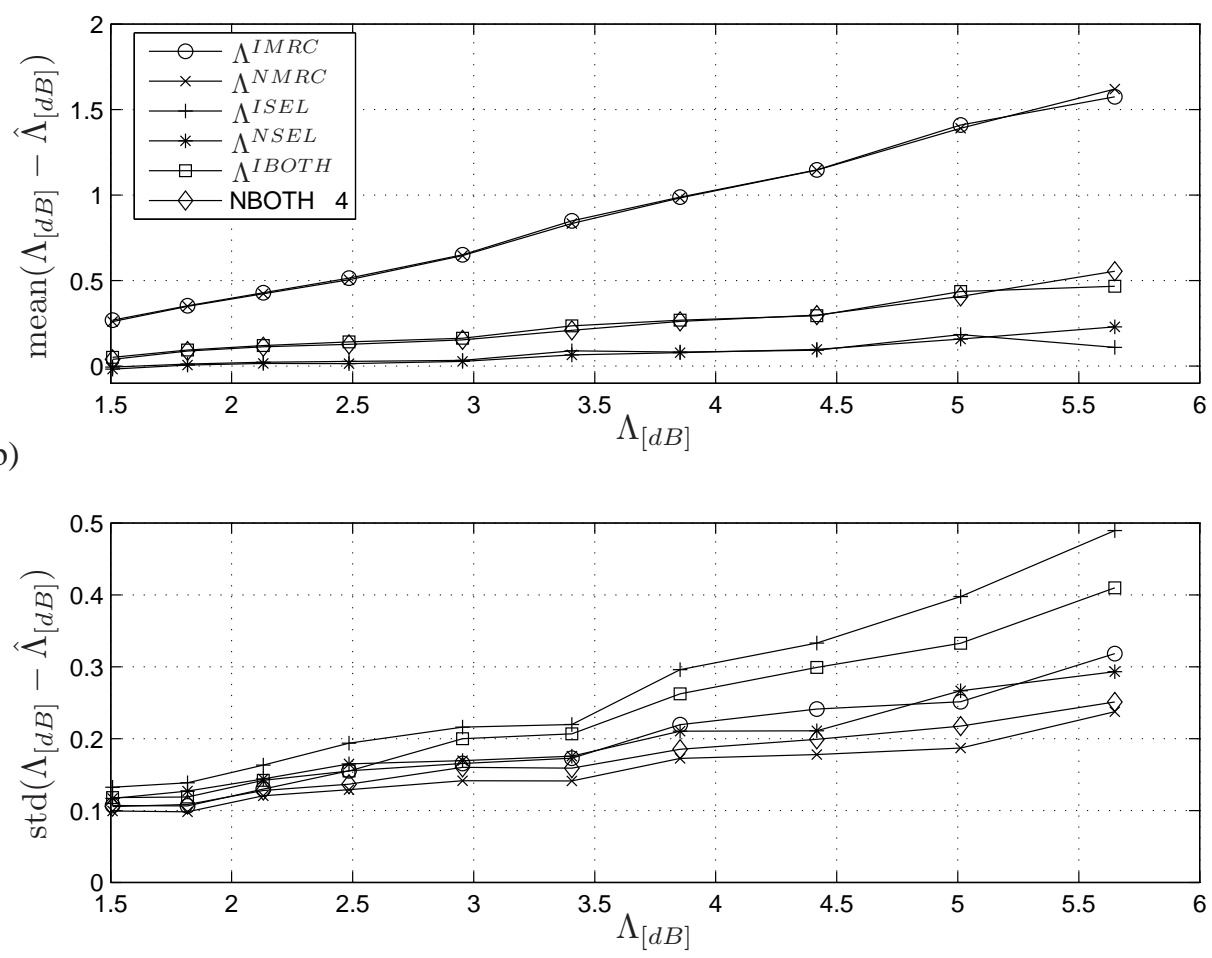

Figure 5.10: Error in noise rise with $64 \mathrm{kbps}$ users. Up to three links per user. a) Average error, b) Standard deviation for single errors. 
independent of different load in different cells. The approximations $\Lambda^{N M R C}, \Lambda^{N S E L}$ and $\Lambda^{N B O T H}$ all use the previous approximation to approximate each user's contribution to the noise rise. By looking at (5.16a), which is repeated below, it is apparent that underestimating the noise rise at time $t-1$ results in underestimating every term in the sum that constitutes the noise rise at time $t$

$$
\Lambda_{j}^{N M R C}(t)=1+\sum_{i=1}^{M} \beta_{i}^{t g t} \frac{g_{i, j}}{\sum_{k \in K_{i}} \frac{g_{i, k}}{\Lambda_{k}^{N M R C}(t-1)}} .
$$

As can be expected, the performance of $\Lambda^{N B O T H}$ and $\Lambda^{I B O T H}$ can be described as an average of the two other assumptions regarding soft or softer handover. The two middle lines in the Figure 5.10a represent $\Lambda^{I B O T H}$ and $\Lambda^{N B O T H}$. The figure shows that they approximate the noise rise with an average error less than $0.5 \mathrm{~dB}$ while maintaining a low standard deviation even for high true noise rise levels, see Figure 5.10b. The slight increase in error as the noise rise increases, may be explained by looking in the load domain. As mentioned in the introduction, even a small difference in load gives a substantial increase in noise rise at high load levels. This also means that a small load error results in a large noise rise error when operating at high load levels. In Figure 5.11 statistics for load expressions are plotted versus true load computed according to (4.2),

$$
L=1-\frac{1}{\Lambda}
$$

where $\Lambda$ is given by the simulator. There is no dramatic increase in error as the load of the system increases in this domain. All the lines in the figure are quite straight but do not have the correct incline. This can best be explained by studying (5.10). Ideally, the expression is a sum over all users in the network. The fact that for example the line representing $L^{I M R C}$ in Figure 5.11 does not have the correct incline is thus an effect of each user's contribution being underestimated and that not all users are considered due to limited path gain reports. All the expressions have a sum over, ideally, all the users, shown in (5.16a), and hence the same reasoning applies. To summarize, there are two reasons why the lines in Figure 5.10 deviates from a straight line for high load levels:

- even a minor error at high loads produces a non-negligible error in the noise rise

- the sum representing the load approximation has a larger error due to many users, all with a minor underestimated contribution.

\section{Hot Spot}

When deriving $\Lambda^{I M R C}, \Lambda^{I S E L}$ and $\Lambda^{I B O T H}$ in Section 5.2.2 the interference power was assumed to be approximately equal in all cells. This is an assumption that is unsuitable for scenarios with hot spots, i.e., when one or several base stations have considerably higher load than the others. As can be seen in Figure 5.12a) all expressions still provide a pretty good average noise rise approximation for moderate noise rise levels. However, under these circumstances $\Lambda^{I S E L}$ is useless due to its errors have a high standard deviation at heavily loaded cells. In order to explain the severe over estimation done by $\Lambda^{I S E L}$ at high 


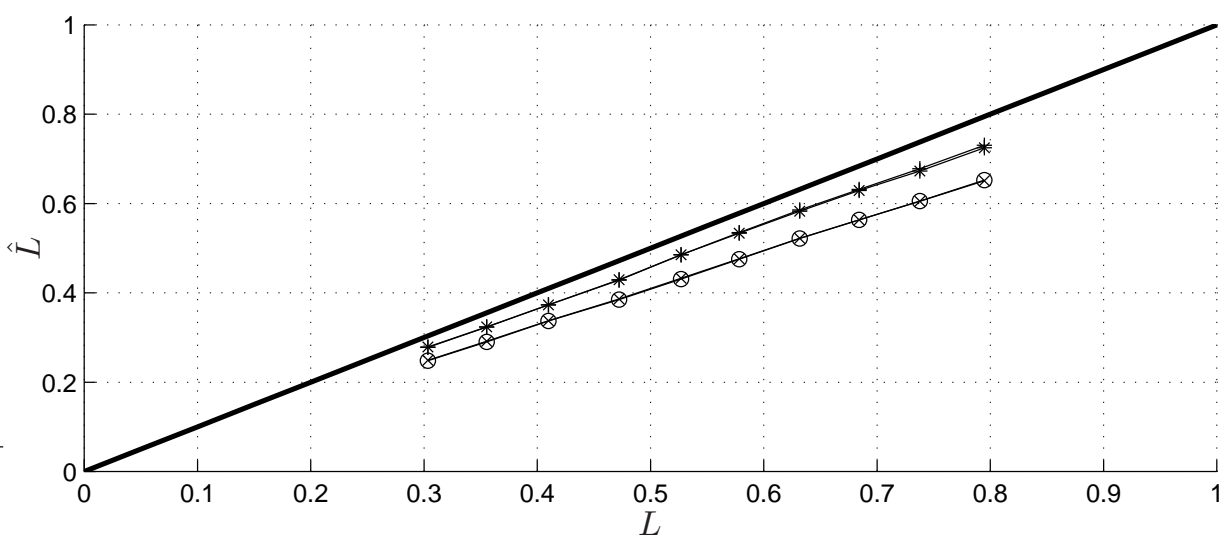

Figure 5.11: Error in uplink load when using $64 \mathrm{kbps}$ users. $L^{I M R C}$ : 'o', $L^{N M R C}$ 'X', $L^{I S E L}$ : '+', $L^{N S E L}$ : '*'. The curve $\hat{L}=L$ is also shown.

loads, study a scenario where user 1 is connected, by soft handover, to base stations $j$ and $\ell$. Since both base stations are in the active set, the path gains from the user to the base stations, i.e., $g_{1, j}$ and $g_{1, \ell}$, cannot differ too much. If $I_{j}^{\text {tot }}$ is much greater than $I_{\ell}^{\text {tot }}$, than the contribution from user 1 is according to (5.6b)

$$
g_{1, j} \frac{\beta_{1}^{t g t}}{\max \left\{\frac{g_{1, j}}{I_{j}^{t o t}}, \frac{g_{1, \ell}}{I_{\ell}^{t o t}}\right\}}=g_{1, j} \frac{\beta_{1}^{t g t}}{g_{1, \ell}} I_{\ell}^{t o t}
$$

while it is reflected in $\Lambda^{I S E L}$ by

$$
g_{1, j} \frac{\beta_{1}^{t g t}}{\frac{1}{I_{j}^{t o t}} \max \left\{g_{1, j}, g_{1, \ell}\right\}}=g_{1, j} \frac{\beta_{1}^{\text {tgt }}}{\max \left\{g_{1, j}, g_{1, \ell}\right\}} I_{j}^{\text {tot }} .
$$

If $I_{j}^{t o t} \gg I_{\ell}^{t o t}$, the load contribution (5.22) is likely highly overestimated using (5.23). This explains $\Lambda^{I S E L}$, s overestimation of the load in highly loaded cells, as illustrated in Figure 5.12. The high standard deviation is a result of the expressions alternating between being an over estimation in the case of different interference levels in neighboring base stations and under estimation in the case of almost equal interference levels. $\Lambda^{N B O T H}$ provides approximations with both low average error and low standard deviation.

\subsubsection{Several Radio Network Controllers}

The knowledge on users' path gains can be even further limited by considering reports from only a subset of the users. As argued in Section 5.2.4, some users will not report their path gain to the RNC they are currently controlled by if they started their session when located in an area served by another RNC. In this section we have used path gain reports according to the scheduling technique referred to as M2 and compared the performance of the expressions defined by (5.18) for different fractions of external users (i.e., users not reporting their path gain to the RNC referred to as $R N C_{1}$ in Section 5.2.4). As a 
a)

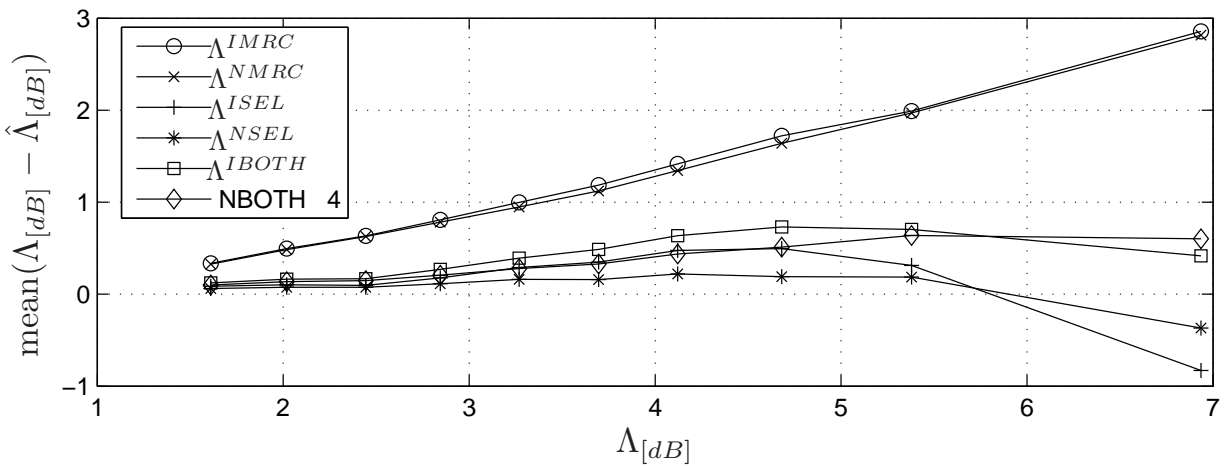

b)

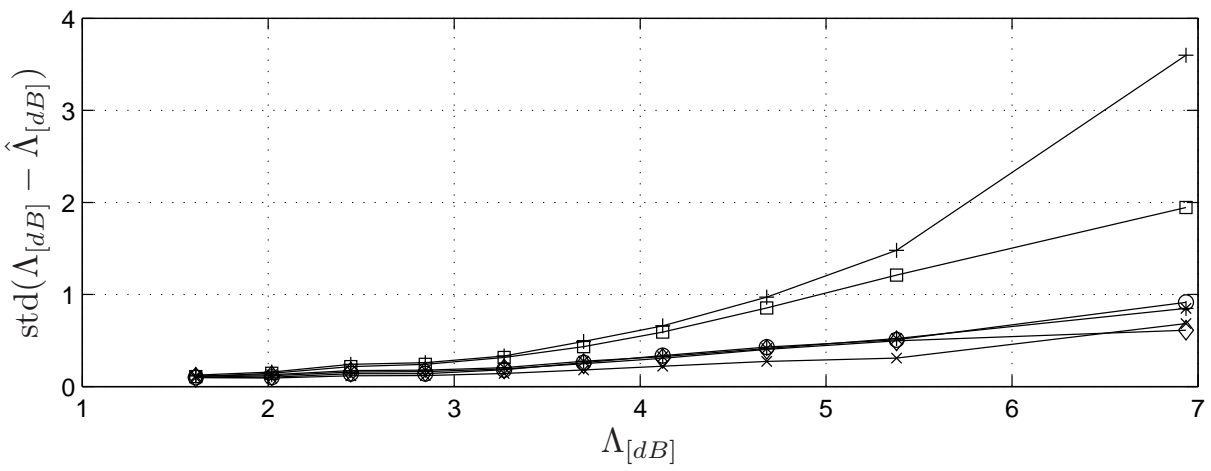

Figure 5.12: Error in noise rise with $64 \mathrm{kbps}$ users. Three links. Hot spot. a) Average approximation error. b) Standard deviation of single approximation errors. 
comparison, statistics for the expression that completely ignores the external users are also studied. Figure 5.13-5.15 show the average estimation error when 10, 20 and 30\% of the users report to another RNC than the one they are closest to, respectively. Clearly, just ignoring that some users are not considered in the expression can be quite drastic. However, when using an expression that incorporates an estimate of the load contribution from the external users (i.e., $\Lambda^{R N C M R C}, \Lambda^{R N C S E L}$ or $\Lambda^{R N C B O T H}$ ), the performance is almost equal to the case where measurement reports from all users are available. A comparison between Figure 5.10b and Figure 5.16 shows that the expressions studied in this section have approximately the same standard deviation as the ones assuming equal background noise power in all cells.

The expressions in (5.13) are not shown here. The problem of not receiving path gain reports from some users can be solved in the same way, i.e., with small messages between different RNCs, without any additional changes to the expressions.

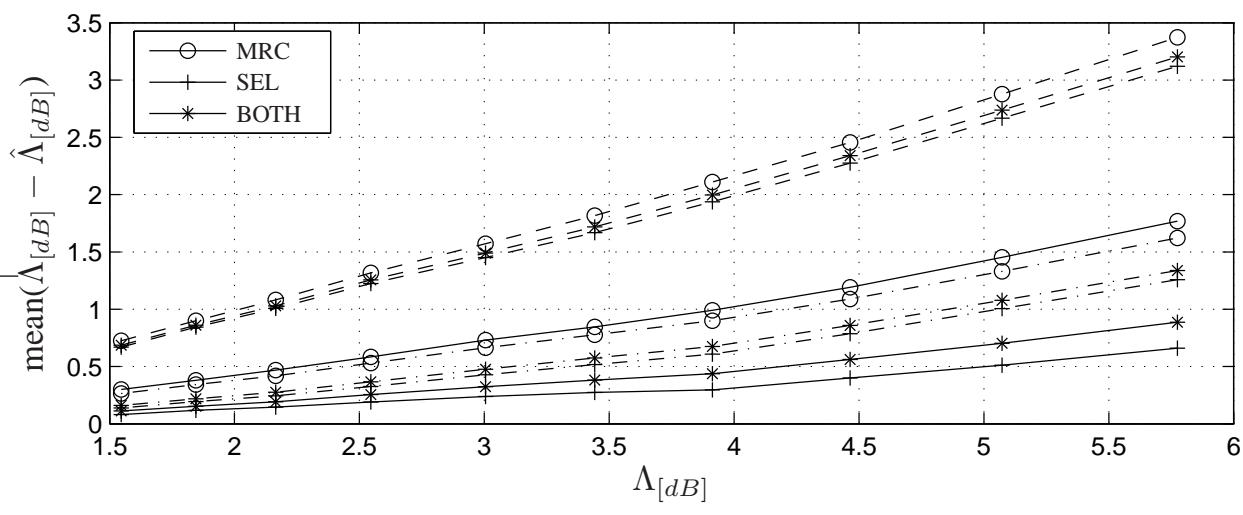

Figure 5.13: Mean errors in noise rise when using two RNCs. Solid: All reports, dashed: Reports in one RNC, dashdotted: $\Lambda^{R N C} .10 \%$ of the users report to another RNC.

\subsection{Summary}

This chapter focused on establishing the noise rise relative load in a practical system. Combining expressions for the total received interference power in different base stations, resulted in a system of nonlinear equations. Two conceptually different methods for approximately solving this system were proposed. The choice of method for solving the system of equations together with different methods for combining information received in different base stations gave a large number of different approximations. By using information gathered in several cells, they all explicitly consider the intercell interference power. This means that they are in fact sensitive to the system's soft capacity. Since the channels' path gain values are considered by the approximations, the approximations are completely robust to any changes in the radio environment, such as different propagation exponents. 


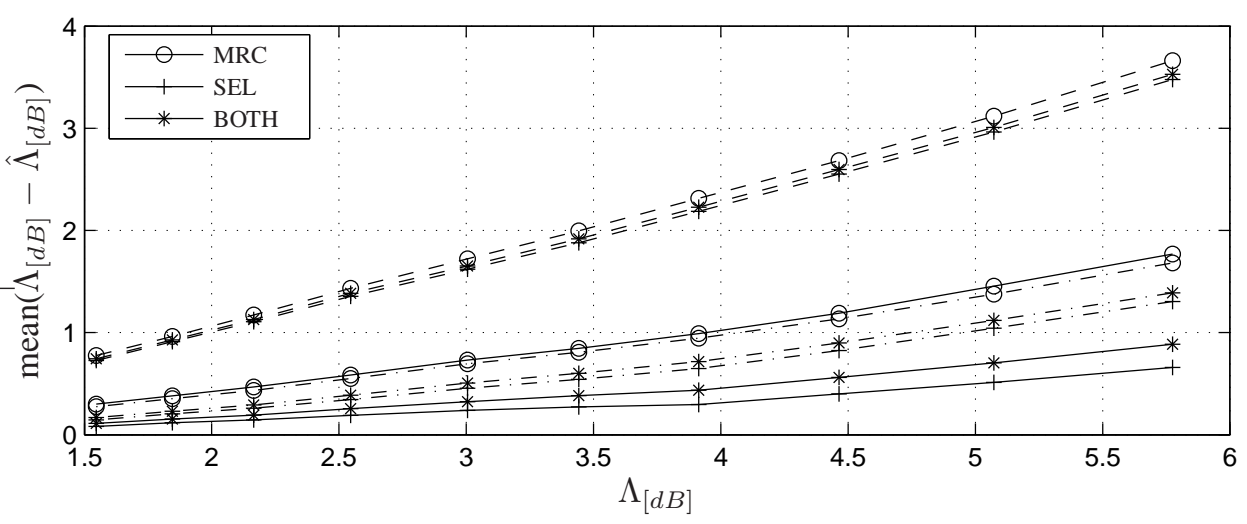

Figure 5.14: Mean errors in noise rise when using two RNCs. Solid: All reports, dashed: Reports in one RNC, dashdotted: $\Lambda^{R N C} .20 \%$ of the users report to another RNC.

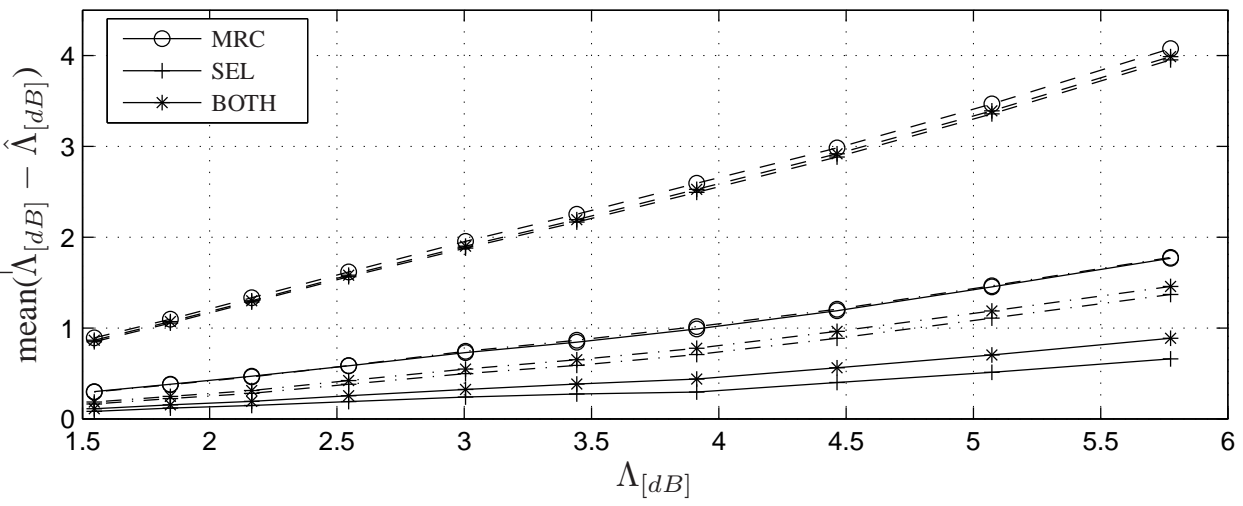

Figure 5.15: Mean errors. Solid:All reports, dashed:Reports in one RNC, dashdotted: $\Lambda^{R N C} .30 \%$ of the users report to another RNC. 


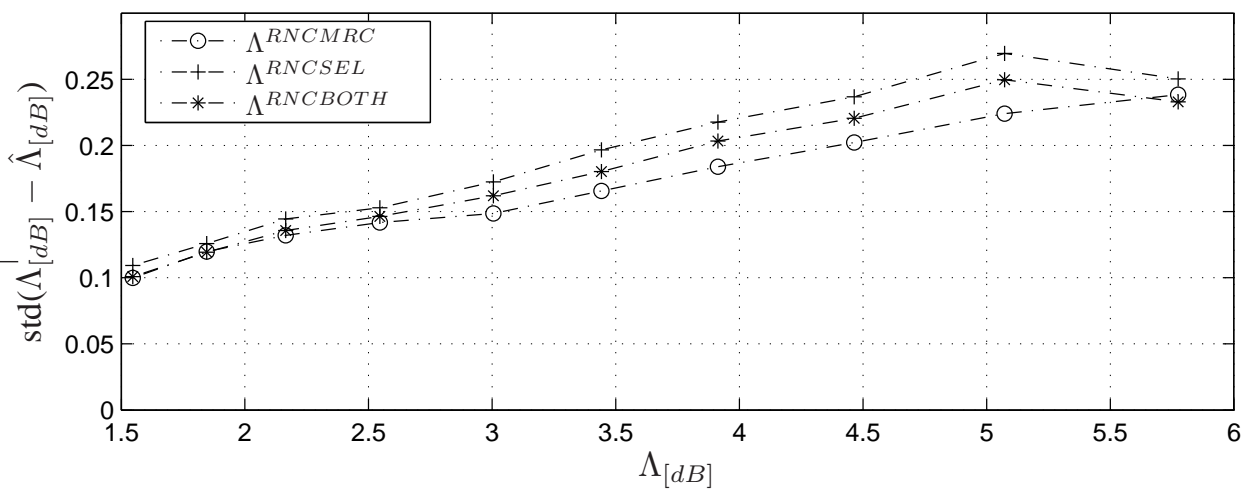

Figure 5.16: Standard deviation of single errors. Dashdotted: $\Lambda^{R N C} .30 \%$ of the users report to another RNC.

As the path gain measurements which the approximations rely on do not include the multi path fading, a technique for approximating the additional intercell interference power that the multi path fading together with fast power control causes. This intercell interference approximation is build on knowledge of the channels impulse responses.

A rather thorough simulation study gave that it is possible to approximate the uplink noise rise with an average error less than $1 \mathrm{~dB}$ and low standard deviation for a noise rise levels even above $8 \mathrm{~dB}$. 


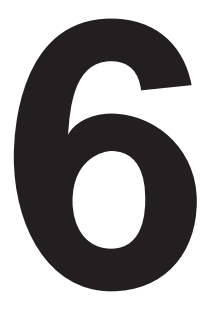

\section{Analyzing Uplink Load}

This chapter studies uplink load from a theoretical perspective. Alternative definitions of uplink load related to the entire system as opposed to just one cell are introduced. Definitions explicitly relating uplink load to existence of a solution to the power control problem of finding appropriate transmission powers for all users are also introduced. A matrix conveniently representing the interconnections between different base stations is introduced. The matrix is then used as a basis for deriving much of the results in this chapter as well as in the following ones. In this chapter the matrix is used to provide links between the uplink load approximations in Chapter 5 and the rather theoretical definitions of uplink load introduced in this chapter. Towards the end, much of the results are condensed into a method for approximating the uplink load in practice. Section 6.2 introduces alternative definitions of uplink load and discusses how the load of a system according to these definitions can be determined. In Section 6.4 relations between different definitions of uplink relative load as well as some of the expressions in the previous chapter are established. All expressions for the uplink load in the previous chapter are derived through approximations of a system of nonlinear equations. Some of the expressions use iterations. Sufficient conditions for convergence of one of the iterations are developed in Section 6.5. Divergence of the iterations will also be related to a lower bound of the uplink load.

Since this is a theoretical study, it is assumed that the system has complete knowledge of all power gains. Furthermore, the users' experienced CTIR will be assumed to equal the target CTIR set by the resource management algorithms. This corresponds to studying the steady state of a perfect power control algorithm. 


\subsection{System Properties}

In this section, a number of properties of a system will be stated in order to simplify the presentation of quantities and results given in the remaining part of the chapter.

\subsubsection{Terminology}

The connections between users and base stations can be conveniently gathered in a matrix.

Definition 6.1 (Link Matrix). The element on row $\ell$ and column $j$ of the link matrix, $K \in \mathbb{R}^{M \times B}$, is defined as

$$
K_{\ell, j} \triangleq\left\{\begin{array}{l}
1, \text { if user } \ell \text { is power controlled by base station } j \\
0, \text { otherwise. }
\end{array}\right.
$$

The sets $K_{\ell}$ can thus be depicted as

$$
K_{\ell}=\left\{j: K_{\ell, j}=1\right\} .
$$

Correspondingly, $c_{j}$, the set of users power controlled by base station $j$, can be defined as

$$
c_{j} \triangleq\left\{\ell: K_{\ell, j}=1\right\} .
$$

Let $N \in \mathbb{R}^{B \times 1}$ be a vector with the base station individual background noise powers. A system with link matrix $K$ and power gain matrix defined according to Definition 3.1 in which the background noise powers in the different cells are given by the vector $N$ and the users have target carrier-to-total interference ratios according to the diagonal matrix $B^{t g t}$ is denoted $\left(B^{t g t}, G, K, N\right)$.

Definition 6.2 (System Feasibility). A system $\left(B^{t g t}, G, K, N\right)$ is feasible if there exist mobile individual finite positive transmission powers such that

$$
\beta_{\ell} \geq \beta_{\ell}^{t g t} \forall \ell
$$

Otherwise, the system is infeasible.

Adopt a terminology stating that a user with links to a number of base stations is said to be connected to those base stations. To quantify the number of base stations mobiles in a network are connected to and power controlled by, strict connectivity and connectivity are introduced.

Definition 6.3 (Strict Connectivity). A system is said to have strict connectivity $k$ if at least one mobile is connected to $k$ base stations.

Definition 6.4 (Connectivity). A system is said to have connectivity $k$ if at least one mobile is power controlled by $k$ base stations.

To exemplify the last two definitions, consider a system with just one user who is in soft handover between two cells, but the power gain to one of the base stations is much higher than that to the other one. The user is thus connected to both base stations 
but power controlled by just one of them. The system then has strict connectivity two but connectivity one. On the other hand, when in softer handover, the user is power controlled by both base stations and the connectivity is then also two.

As concluded in Example 4.3, the interference power a user causes in base station $j$ is proportional to the ratio between $g_{i, j}$ and $g_{i, K_{i}}$ in a system with connectivity one. In connection with the example, these ratios were gathered in a matrix. Here, a similar matrix, the relative power gain matrix, is defined.

Definition 6.5 (Relative Power Gain Matrix). The element on row $i$ and column $j$ of the relative power gain matrix, $Z \in \mathbb{R}^{M \times B}$, is at time $t$

$$
z_{i, j}(t) \triangleq \frac{g_{i, j}(t)}{\sum_{k \in K_{i}} g_{i, k}(t)}
$$

\subsubsection{Interference Power Expression}

A system with connectivity one is more simple to analyze theoretically. All mobiles essentially communicate with one base station in a system with connectivity one. For example, it is possible to establish a matrix expression for the received interference powers.

Combining (5.1) and (5.2) yields (suppressing the time index, $t$ )

$$
I_{j}^{t o t}=N_{j}+\sum_{i=1}^{M} p_{i} g_{i, j}
$$

If user $i$ is solely connected to base station $K_{i}$,

$$
\beta_{i}=\frac{C_{i, K_{i}}}{I_{K_{i}}^{t o t}}=p_{i} \frac{g_{i, K_{i}}}{I_{K_{i}}^{t o t}}
$$

where the first equality is the definition of $\beta_{i}$ (see (3.4)). Solving for $p_{i}$ and inserting it into the above expression for $I_{j}^{t o t}$ yield

$$
I_{j}^{\text {tot }}=N_{j}+\sum_{i=1}^{M} \beta_{i} \frac{g_{i, j}}{g_{i, K_{i}}} I_{K_{i}}^{\text {tot }}=N_{j}+\sum_{i=1}^{M} \beta_{i} z_{i, j} I_{K_{i}}^{\text {tot }} .
$$

The above sum over users can be split into two sums,

$$
I_{j}^{\text {tot }}=N_{j}+\sum_{k=1}^{B} \sum_{i \in c_{k}} \beta_{i} z_{i, j} I_{K_{i}}^{\text {tot }}=N_{j}+\sum_{k=1}^{B} \sum_{i \in c_{k}} \beta_{i} z_{i, j} I_{k}^{\text {tot }} .
$$

The core of the framework developed here is the system matrix.

Definition 6.6 (System Matrix). The element on row $k$ and column $j$ of the system matrix, $L \in \mathbb{R}^{B \times B}$, is

$$
L_{k, j}(t) \triangleq \sum_{i \in c_{k}} \beta_{i}^{t g t}(t) z_{i, j}(t)
$$


Given the link matrices $K$, relative power gain matrix $Z$ and the users' target CTIR values in a diagonal matrix $B^{t g t}$,

$$
L=K^{T} B^{t g t} Z
$$

With the purpose of expressing the total interference powers in (6.1) in vector form, assume perfect power control. This justifies substituting $\beta_{i}$ by $\beta_{i}^{t g t}$ which makes it possible to write (6.1) as

$$
I_{j}^{t o t}=\sum_{k=1}^{B} L_{k, j} I_{k}^{t o t} .
$$

The vector expression corresponding to (6.1) is then

$$
I^{t o t}=L^{T} I^{t o t}+N \Leftrightarrow I^{t o t}=\left(E-L^{T}\right)^{-1} N .
$$

\subsection{System Load}

\subsubsection{System Noise Rise Relative Load}

Even though a relative load per cell is interesting from a practical point of view since it is used with advantage in the resource management, a load related to the entire system is equally interesting from a theoretical point of view. One of the conclusions in Chapter 4 is that the uplink noise rise is a natural load measure in the uplink. Therefore, the following definition of system load is a natural one to make.

Definition 6.7 (System Noise Rise Relative Load). The system noise rise relative load is defined as

$$
L_{s} \triangleq \max _{j} L_{j}^{n r}
$$

where $L_{j}^{n r}$ is the uplink noise rise relative load in cell $j$ according to Definition 4.2.

The expressions for uplink noise rise relative load in Chapter 5 can thus be used to approximate the system noise rise relative load, e.g.,

$$
\begin{aligned}
L_{s}^{I M R C} & \triangleq \max _{j} L_{j}^{I M R C} \\
L_{s}^{I S E L} & \triangleq \max _{j} L_{j}^{I S E L} .
\end{aligned}
$$

\subsubsection{Feasibility Relative Load}

Yet another definition of load is one based on the system as a whole, i.e., not as consisting of a number of small systems (cells). The load of a system can then be explicitly related to existence of a solution to the system's power control problem of finding appropriate transmission powers to satisfy all users' requirements on carrier-to-interference ratios.

The distance to infeasibility according to Defintion 6.2 was expressed by Herdtner and Chong (2000) as a feasibility margin. Later, feasibility margin was related to feasibility relative load by Gunnarsson (2000). In these references the quantities were expressed in terms of CIR. Below, these quantities are redefined in terms of CTIR instead. 
Definition 6.8 (Feasibility Margin). The feasibility margin, $\Gamma_{m}$, of a cellular system, $\left(B^{t g t}, G, K, N\right)$, is defined as

$$
\Gamma_{m} \triangleq \sup \left\{x \in \mathbb{R}:\left(x B^{t g t}, G, K, N\right) \text { is feasible }\right\} .
$$

Clearly, $\Gamma_{m}>1$ corresponds to a feasible system. The interpretation of $\Gamma_{m}$ is that it is the largest factor by which all users' target CTIR values can be scaled without losing feasibility. The relation to the, within automatic control well known quantity, gain margin is obvious.

The alternative load measure can now be defined in terms of the feasibility margin.

Definition 6.9 (Feasibility Relative Load). The feasibility relative load, $L_{f}$, of a system $\left(B^{t g t}, G, K, N\right)$ is defined as

$$
L_{f} \triangleq \frac{1}{\Gamma_{m}}=\inf \left\{\mu \in \mathbb{R}:\left(\frac{1}{\mu} B^{t g t}, G, K, N\right) \text { is feasible }\right\} .
$$

System feasibility is a necessary condition for stability of distributed power control (Gunnarsson, 2000; Herdtner and Chong, 2000; Yates, 1995; Zander, 1993). Note that system infeasibility together with unlimited uplink transmission powers imply an infinite received interference power in some cell. This, in turn, implies that the uplink noise rise relative load equals one in at least one cell. Conversely, the system is feasible if the system noise rise relative load in Definition 6.7 is less than one.

The following theorem is used here merely to give some intuition for Definition 6.8 and Definition 6.9. The result is the same and the proof similar to those given by Zander (1993), only this is applicable to the uplink as opposed to the original theorem which is derived for the downlink.

\section{Theorem 6.1}

A noise-less single-service system with connectivity one, $\left(\beta_{0} E, G, K, \mathbf{0}^{B}\right)$, has a maximum achievable carrier-to-total-interference ratio of

$$
\beta_{0}^{*}=\max \left\{\beta_{0} \mid I_{j}^{t o t}>0 \forall j \in[1, B], \beta_{i} \geq \beta_{0} \forall i \in[1, M]\right\} .
$$

Furthermore, the maximum is given by

$$
\beta_{0}^{*}=\frac{1}{\bar{\lambda}\left(Z^{T} K\right)},
$$

where $Z$ is the system's relative power gain matrix.

Proof: According to 6.2, the system matrix in a single service scenario is

$$
L=\beta_{0} K^{T} E Z,
$$

where $\beta_{0}$ is the target CTIR of the only service provided and $E$ is the identity matrix in $\mathbb{R}^{M \times M}$. Applying (6.3) to a noise-less scenario yields

$$
Z^{T} K I^{t o t}=\frac{1}{\beta_{0}^{*}} I^{t o t}=\lambda I^{t o t} .
$$

The smallest real $\lambda$ which gives a positive real solution $I^{\text {tot }}$ is the greatest eigenvalue of $K Z^{T}, \bar{\lambda}\left(Z^{T} K\right)$, according to theory for positive matrices, see Appendix A.2. Since a larger $\beta_{0}^{*}$ corresponds to smaller eigenvalues of $K Z^{T}, \frac{1}{\beta_{0}^{*}}=\bar{\lambda}\left(Z^{T} K\right)$. 
The same result applies also for a system with noise as Theorem 6.2 will show. Given the above result that a maximum $\beta_{0}$ does exist, the intuitive choice of feasibility margin and feasibility relative load in a single service system,

$$
\Gamma_{m}=\frac{\beta_{0}^{*}}{\beta_{0}} \text { and } L_{f}=\frac{\beta_{0}}{\beta_{0}^{*}},
$$

are true if the system has connectivity one.

The definition of feasibility relative load above is similar to Definition 4.3. They are, however, not equal since Definition 6.9 uses target carrier-to-total-interference $\left(\beta^{\text {tgt }}\right)$ ratios instead of target carrier-to-interference ratios $\left(\gamma^{t g t}\right)$ but most of all, Definition 4.3 is concerned with the downlink. Note that $L_{f}$ has the desirable properties that $L_{f} \geq 0$ and that a system is feasible if and only if $L_{f}<1$.

\subsection{Estabilishing the Feasibility Relative Load}

This section is devoted to finding an expression for the feasibility load. Connectivity one and higher connectivity will be handled in separate subsections.

\subsubsection{Connectivity One}

The system property $L_{f}$ can be found in systems with connectivity one by using Theorem 6.2 below. For convenience introduce

$$
\bar{\lambda}(L) \triangleq \max \operatorname{eig}(L),
$$

for the maximum eigenvalue of the matrix $L$.

\section{Theorem 6.2 (Feasibility Relative Load)}

In a system $\left(B^{\text {tgt }}, G, K, N\right)$ with connectivity one, the feasibility uplink relative load is

$$
L_{f}=\bar{\lambda}(L) .
$$

Proof: According to Definition 6.9, the feasibility relative load is the infimum $\mu$ such that $\left(\frac{1}{\mu} B, G, K, N\right)$ is feasible. The system is feasible if there are finite positive transmission powers such that $\beta_{i}^{t g t} \leq \beta_{i}$. Since $\beta_{i}>0$ and $g_{i, K_{i}}>0$,

$$
p_{i}=\frac{\beta_{i}}{g_{i, K_{i}}} I_{K_{i}}^{\text {tot }}>0 \Rightarrow I_{K_{i}}>0 .
$$

The feasibility relative load is thus the smallest real $\mu$ yielding a positive solution $I^{\text {tot }} \succ 0$ to

$$
I^{t o t} \succeq \frac{1}{\mu} L^{T} I^{t o t}+N
$$

Let us first consider a noise-free scenario, i.e., $N=0$,

$$
I^{t o t} \succeq \frac{1}{\mu} L^{T} I^{t o t} \Leftrightarrow L^{T} I^{t o t} \preceq \mu I^{t o t}
$$


where the inequalities should be interpreted element wise. According to Perron-Frobenius theory for positive matrices, the smallest real $\mu$ such that there is an $I^{t o t}=I_{0}^{t o t}>0$ that satisfies the inequality is the maximum eigenvalue to $L^{T}$, see Appendix A.2. Now, consider a scenario with target CTIR values $\frac{1}{\mu+\epsilon} \beta_{i}^{t g t}, \epsilon>0$. The inequality is then strictly satisfied,

$$
I_{0}^{\text {tot }} \succ \frac{1}{\mu+\epsilon} L^{T} I_{0}^{t o t} \Leftrightarrow I_{0}^{t o t}=\frac{1}{\epsilon+\mu} I_{0}^{t o t}+\Delta,
$$

where $\Delta \succ 0$. Therefore, it is always possible to choose a $k>0$ such that

$$
k \Delta \succeq N .
$$

To conclude, the smallest $\mu+\epsilon$ such that there is a positive solution $I^{\text {tot }}$ to (6.4) is $\bar{\lambda}\left(L^{T}\right)$.

The feasibility relative load is obviously related to the users' target CTIR and the intercell interference they cause, even though often not as simple as in the case with the noise rise relative load. Example 6.1 studies a simple single service scenario with just two cells.

\section{Example 6.1: Feasibility Relative Load, Single Service}

Consider a system consisting of two cells in which the only service provided requires a target CTIR of $\beta_{0}$. If soft handover is not utilized, the system matrix is

$$
L=\left(\begin{array}{cc}
M_{1} \beta_{0} & \beta_{0} \sum_{i \in c_{1}} z_{i, 2} \\
\beta_{0} \sum_{i \in c_{2}} z_{i, 1} & M_{2} \beta_{0}
\end{array}\right),
$$

where $M_{1}$ and $M_{2}$ are the number of users connected to base station one and two, respectively. Assume an average relative power gain per cell of 0.4 , i.e.,

$$
\frac{1}{M_{1}} \sum_{i \in c_{1}} z_{i, 2}=\frac{1}{M_{2}} \sum_{i \in c_{2}} z_{i, 1}=0.4 .
$$

According to Theorem 6.2, the feasibility relative load is the maximum eigenvalue of the system matrix. Given a target maximum feasibility relative load, $L_{f}^{\max }$, an upper bound on $M_{2}$ is

$$
M_{2} \leq \frac{L_{f}^{\max }-M_{1} \beta_{0}}{\beta_{0}\left(L_{f}^{\max }-\left(1-0.4^{2}\right) M_{1} \beta_{0}\right)} L_{f}^{\max } .
$$

This gives the relation between the system capacity and the capacity of cell one according to Figure 6.1 when $\beta_{0}$ equals 0.1 or 0.3 . Choosing a smaller $\beta_{0}$ yields smaller maximal total capacity. On the other hand, a smaller $\beta_{0}$ enables admitting more users. This result concurs with the statement that higher throughput comes with less fairness, i.e., by giving more resources to a few users the throughput is increased, but more users will experience poor or no service at least momentarily.

Another observation is that the maximum capacity is reached when the resources are shared between the base stations. This is a result of less interference when fewer users are connected to the same base station. 


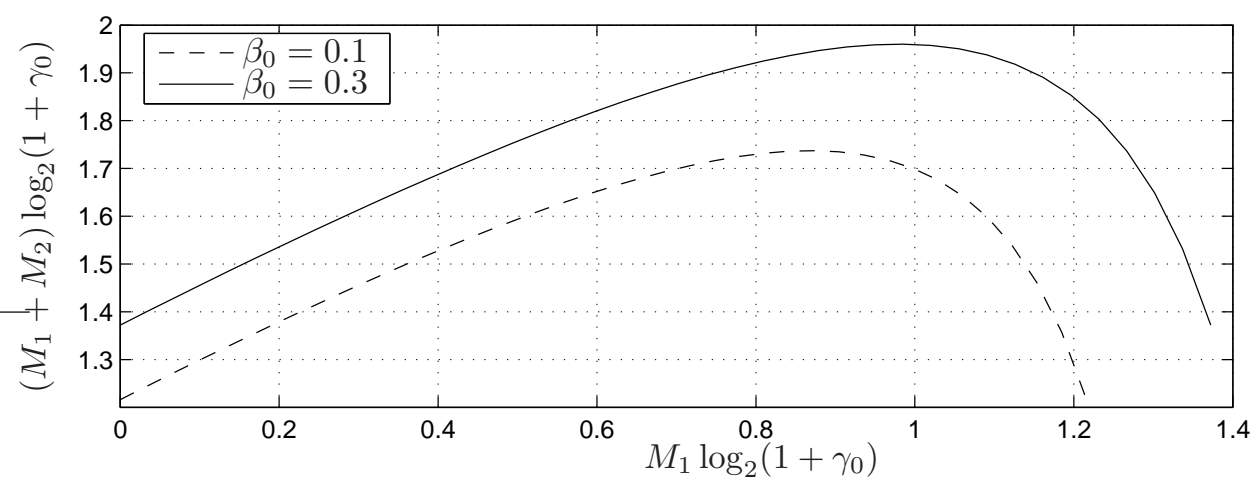

Figure 6.1: Maximum combined capacity in cell one and two, as a function of the capacity in cell one in Example 6.1. $L_{f}^{\max }=0.8$.

\section{Example 6.2: Feasibility Relative Load, Multi Service}

Consider a system with two users and two base stations in which user one is controlled by base station one and user two by base station two. The link matrix and the power gain matrix are

$$
K=\left(\begin{array}{ll}
1 & 0 \\
0 & 1
\end{array}\right) \text { and } G=\left(\begin{array}{cc}
0.8 & 0.32 \\
g_{2,1} & 0.7
\end{array}\right)
$$

respectively. According to Theorem 6.2, the feasibility relative load is the maximum eigenvalue of $B^{t g t} Z$.

The upper bound on $\beta_{2}^{t g t}$ is therefore

$$
\beta_{2}^{t g t} \leq \frac{\beta_{1}^{t g t}-L_{f}}{\beta_{1}^{t g t}\left(1-z_{1,2} z_{2,1}\right)-L_{f}} L_{f} .
$$

Figure 6.2 shows the relation between $\beta_{1}^{t g t}$ and $\beta_{2}^{\text {tgt }}$ such that the feasibility relative load is 0.8 for two different values of $g_{2,1}$. The figure shows that we can allow a higher values for $\beta_{2}^{t g t}$ for a given $\beta_{1}^{t g t}$ when $g_{2,1}$ is smaller. A $g_{2,1}=0$ would give a straight horizontal line at $\beta_{2}^{t g t}=L_{f}$.

Remark 6.1. Since soft handover can only decrease the uplink load, a $L_{f}$ found using Theorem 6.2 in a system with connectivity one will be an upper bound of the load in the corresponding system with soft handover.

An application of Theorem 6.2 is to show that $L_{f}$ equals the natural definition of feasibility relative load in a single service scenario

$$
L_{f}=\bar{\lambda}\left(K^{T} B^{t g t} Z\right)=\bar{\lambda}\left(\beta_{0}^{t g t} K^{T} Z\right)=\beta_{0}^{t g t} \bar{\lambda}\left(K^{T} Z\right)=\beta_{0} \lambda^{*}=\frac{\beta_{0}^{t g t}}{\beta_{0}^{*}} .
$$




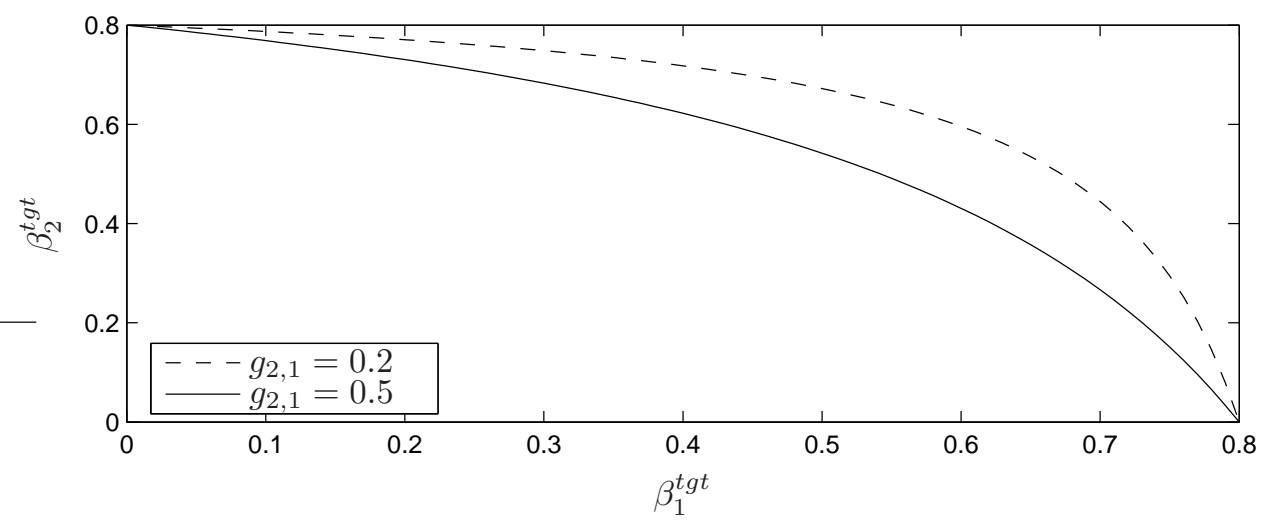

Figure 6.2: Relation between $\beta_{1}^{\text {tgt }}$ and $\beta_{2}^{\text {tgt }}$ such that $L_{f}=0.8$ in Example 6.2.

\subsubsection{Higher Connectivity}

A special case of connectivity higher than one is that all users are power controlled by all base stations. Hanly (1996) shows that, in this special case, the system is feasible if

$$
\sum_{i=1}^{M} \beta_{i}^{t g t} \leq B
$$

Using Defintion 6.9 of feasibility relative load yields

$$
L_{f}=\frac{\sum_{i=1}^{M} \beta_{i}^{t g t}}{B} .
$$

In the general case of higher connectivity, where the users are power controlled by arbitrarily many base stations, a matrix expression can no longer be obtained for the received interference powers. There are also numerous combinations of methods for combining signals received in different base stations. If all signals are combined using maximum ratio combining the received interference powers are the solution to (5.6a), i.e.,

$$
I_{j}^{t o t}=N_{j}+\sum_{i=1}^{M} \beta_{i}^{t g t} \frac{g_{i, j}}{\sum_{k \in K_{i}} \frac{g_{i, k}}{I_{k}^{t o t}}}, j=1,2, \ldots, B .
$$

Introduce the functions

$$
f_{j}\left(B^{t g t}, G, K, N, I\right) \triangleq N_{j}(t)+\sum_{i=1}^{M} \beta_{i}^{t g t}(t) \frac{g_{i, j}(t)}{\sum_{k \in K_{i}} \frac{g_{i, k}(t)}{I_{k}^{t o t}(t)}}, j=1,2, \ldots, B .
$$

The idea is to approximate these functions with linear functions about a point, $I_{0}^{\text {tot }} \in \mathbb{R}^{B}$, and then use the theory for positive matrices that was previously used in the proof of Theorem 6.2. Let $\hat{L}\left(I_{0}^{t o t}\right)$ be the jacobian matrix of $f$. Each element $\hat{L}_{\ell, j}\left(I_{0}^{t o t}\right)$ of $\hat{L}\left(I_{0}^{t o t}\right)$ is defined by

$$
\hat{L}_{\ell, j}\left(I_{0}^{t o t}\right) \triangleq \frac{\partial f_{j}\left(B^{t g t}, G, K, N, I^{t o t}\right)}{\partial I_{\ell}^{t o t}}=\sum_{i \in c_{\ell}} \beta_{i}^{t g t} \frac{g_{i, j}}{\left(\sum_{k \in K_{i}} \frac{g_{i, k}}{I_{k}^{t o t}}\right)^{2}} \frac{g_{i, \ell}}{I_{\ell}^{t o t}}{ }^{2} j, \ell=1,2, \ldots, B .
$$


Based on this linear approximation, the feasibility relative load with arbitrarily connectivity can be approximated as

$$
L_{f} \approx \bar{\lambda}\left(\hat{L}\left(I_{0}^{t o t}\right)\right) .
$$

\subsection{Relative Load Comparisons}

In a single cell system the relative load according to Definition 4.2, Definition 6.7 and Definition 6.9 are the same according to the following theorem.

\subsubsection{Connectivity One}

\section{Theorem 6.3}

In a single cell system with multiple services, the relative load according to Definition 4.2, Definition 6.7 and Definition 6.9 equals

$$
L_{f}=\sum_{i=1}^{M} \beta_{i}^{t g t}=L^{n r}=L_{s}
$$

Proof: It is shown in Example 4.1 that the uplink noise rise relative load in a single cell system is the sum of the users' target CTIR. Since it is a single cell system, this must trivially be the system noise rise relative load as well.

When it comes to the feasibility relative load, first note that all relative power gains are one in a single cell system. The system matrix is then simply the sum of users' target CTIR,

$$
L=\sum_{i=1}^{M} \beta_{i}^{t g t} .
$$

Thus, the feasibility relative load equals the noise rise relative load in single cell system. load.

In a multi cell system, the feasibility relative load is smaller than the noise rise relative

\section{Theorem 6.4}

In a feasible noise-flat system with connectivity one,

$$
L_{f} \leq L_{s}
$$

Proof: Let us first make the following transformation

$$
L_{f} \leq L_{s} \Leftrightarrow \frac{1}{1-L_{f}} \leq \frac{1}{1-L_{s}}=\max _{j} \Lambda_{j} .
$$

Therefore, it is sufficient to prove that $\frac{1}{1-L_{f}} \leq \max _{j} \Lambda_{j}$. Similar to the maximum eigenvalue, introduce

$$
\underline{\lambda}(A) \triangleq \min |\operatorname{eig} A|,
$$


for the eigenvalue of $A$ with the smallest magnitude. In a noise-flat system the maximum noise rise is

$$
\max _{j} \Lambda_{j}=\max _{j}\left(E-L^{T}\right)^{-1} \mathbf{1}=\left\|\left(E-L^{T}\right)^{-1}\right\|_{\infty} \geq \bar{\lambda}\left(\left(E-L^{T}\right)^{-1}\right),
$$

where the second equality is true since the system is feasible, see Appendix A.2. The above inequality is true since, according to Skogestad and Postlethwaite (1996),

$$
\bar{\lambda}(A) \leq\|A\| .
$$

It remains to prove that $\bar{\lambda}\left(\left(E-L^{T}\right)^{-1}\right)$ equals $1 /\left(1-L_{f}\right)$,

$$
\bar{\lambda}\left(\left(E-L^{T}\right)^{-1}\right)=\frac{1}{\underline{\lambda}\left(\left(E-L^{T}\right)\right)}=\frac{1}{1-\bar{\lambda}\left(L^{T}\right)}=\frac{1}{1-L_{f}} .
$$

Other results, which are perhaps more useful in practice, are the following two theorems. Introduce

$$
L_{j}^{l o c a l} \triangleq \sum_{\ell \in c_{j}} \beta_{\ell}^{t g t}
$$

for the load users connected to cell $j$ cause in the own cell.

\section{Theorem 6.5 (Bound on Feasibility Relative Load)}

Consider a noise-flat system with connectivity one. The feasibility relative load, $L_{f}$, is bounded below and above by

$$
\max _{j} L_{j}^{\text {local }} \leq L_{f} \leq \max _{j} L_{j}^{I M R C} .
$$

Proof: See Appendix A.4.

Corollary 6.1 (Sufficient Condition for Stability)

A noise-flat system with connectivity one is feasible if

$$
\max _{j} L_{j}^{I M R C}<1 .
$$

Proof: Follows from Theorem 6.5 and the fact that a system is feasible if $L_{f}<1$.

\section{Corollary 6.2 (Necessary Condition for Stability)}

A necessary condition for feasibility in a noise-flat system with connectivity one is

$$
L_{j}^{\text {local }}<1 \forall j
$$

Proof: Follows from the fact that a system is feasible only if $L_{f}<1$ and Theorem 6.5.

Remark 6.2. This result shows that, in case of connectivity one, a $L_{s}^{I M R C}<1$ is associated with a feasible uplink power control problem. Allowing soft handover (corresponding to connectivity higher than one) can only yield lower uplink load and therefore a $L_{s}^{I M R C}<1$ found without soft handover also proves stability of a system with soft handover. 
Remark 6.3. In the above calculations we used expressions derived under an assumption of maximum ratio combining, any of the other two assumptions, soft handover or correct combination, might just as well have been made since these three are exactly the same when each user is allowed no more than one link.

\subsubsection{Higher Connectivity}

The maximum noise rise relative load can in fact be bounded by similar limits when soft handover is utilized. The following bounds on the uplink noise rise relative load in a noiseflat system with any connectivity should be compared to those given by Theorem 6.5.

\section{Theorem 6.6}

In a noise-flat system with perfect power control, the uplink noise rise relative load is bounded by

$$
\max _{j} L_{j}^{l o c a l} \leq \max _{j} L_{j}^{n r} \leq \max _{j} L_{j}^{I S E L}
$$

Proof: The lower bound is obvious since it corresponds to neglecting the inter-cellinterference. Regarding the upper bound assume, without loss of generality, that base station one is the base station with highest interference power. This is also the base station with highest noise rise relative load since the background noise power is equal in all base stations. Consider the derivation of $L_{1}^{I S E L}$,

$$
I_{1}^{t o t} \leq N_{1}+\sum_{\ell=1}^{M} \beta_{\ell}^{t g t} \frac{g_{\ell, 1}}{\max _{k} \frac{K_{\ell, k} g_{\ell, k}}{I_{k}^{t o t}}} \leq N_{1}+\sum_{\ell=1}^{M} \beta_{\ell}^{t g t} \frac{g_{\ell, 1}}{\max _{k} \frac{K_{\ell, k} g_{\ell, k}}{I_{1}^{t o t}}}
$$

Using only selection combining justifies the first inequality above since this yields a possible under estimation of the system's ability to utilize the received signals. Because of the second inequality, solving for $L_{1}^{I S E L}(t)$ results in an over estimation of the true $L_{1}^{n r}(t)$. Assuming perfect power control justifies using $\beta_{\ell}^{t g t}(t)$ instead of the actual received CTIR, $\beta_{\ell}(t)$.

In the special case of a system with connectivity one, $L_{j}^{I S E L}=L_{j}^{I M R C} \forall j$. Therefore $L^{I M R C}$ can also be used to bound the true uplink noise rise relative load in a system with connectivity one.

\subsection{Convergence of Fix Point Iterations}

The stationary points of the iterations in Section 5.2.3, if they exist, are approximations of the uplink noise rise. This section provides sufficient conditions for convergence of iterations applied to (5.16a) as well as a criteria for making system infeasibility probable. The iterations may be fix point iterations or single updates using (5.16a) with the assumption of constant power gain values and target carrier-to-total-interference ratios during the time it takes the iterations to converge. Fix point iterations may be implemented as the algorithm below. 


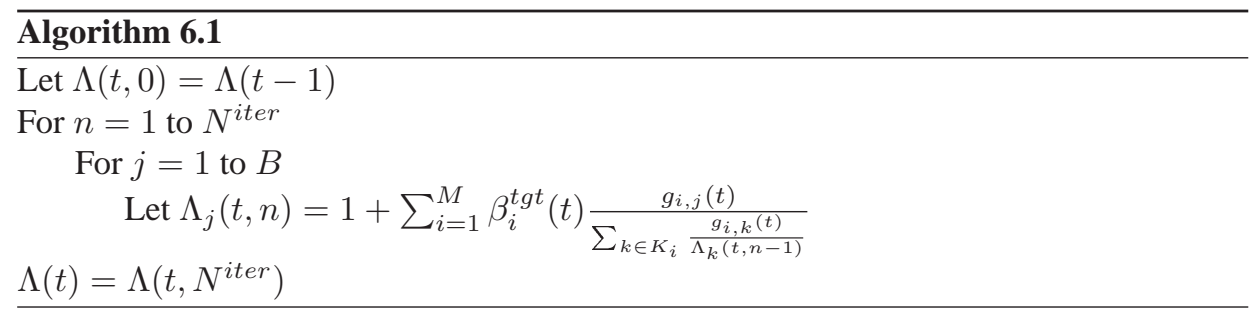

\subsubsection{Connectivity One}

Consider a system without soft handover, i.e., a system with strict connectivity one.

\section{Theorem 6.7 (Convergence of Fix Point Iterations)}

Algorithm 6.1 will converge to the true noise rise if applied to a feasible noise-flat system with connectivity one.

Proof: See Appendix A.5.

Algorithm 6.1 therefore converges to true noise rise when applied in a feasible system with connectivity one and perfect power control, regardless of the initialization point.

\subsubsection{Higher Connectivity}

\section{Theorem 6.8 (Convergence of Fix Point Iterations)}

When Algorithm 6.1 is initialized with $a \Lambda(t, 0) \leq \Lambda$ and applied to a noise-flat feasible system, it converges to a fixed point bounded by the true noise rise.

Proof: See Appendix A.6

Remark 6.4. The definitions of load given in this chapter have been concerned with the entire system. The expressions in Chapter 5, however, provide one relative load measure per cell. From a theoretical point of view, a measure of the load related to the entire system is equally interesting since nowhere is the load allowed to exceed one if the system is to be stable.

\subsection{Approximating Uplink Load in Practice}

The results of the theorems in the previous section are summarized in Algorithm 6.2 which is a recipe for approximating the noise rise relative load in a noise-flat system. Note that in the case of connectivity one, $L_{j}^{I S E L}$ equals $L_{j}^{I M R C}$.

\subsection{A Simulation Example}

Figure 6.3 exemplifies the bounds provided in Theorem 6.5 and Theorem 6.6. Clearly $L_{f}$ is always within the bounds given in Theorem 6.5. Considering the upper bounds given in Theorem 6.6, on the other hand, they are occasionally violated by the maximum noise 
Algorithm 6.2 Approximating Uplink Load

- System with connectivity one

- Check if $\max _{j} L_{j}^{I M R C}$ in (5.10) is less than one.

- If so, approximate the noise rise using Algorithm 6.1 which is guaranteed to converge to the true noise rise levels.

- Transform noise rise to noise rise relative load.

- System with connectivity greater than one

- Check if $\max _{j} L_{j}^{I S E L}$ in (5.11) is less than one.

- If so, approximate the noise rise using Algorithm 6.1 which is guaranteed to converge to a fix point bounded above by the true noise rise.

- Transform noise rise to noise rise relative load.

rise relative load. This is so, even though the TX increase compensation introduced in Section 5.5 is applied. The reason being simply that power control errors are introduced in the simulations that the figure is based on. Note that the system simulated has connectivity one, which implies that the upper bound in both theorems are equal.

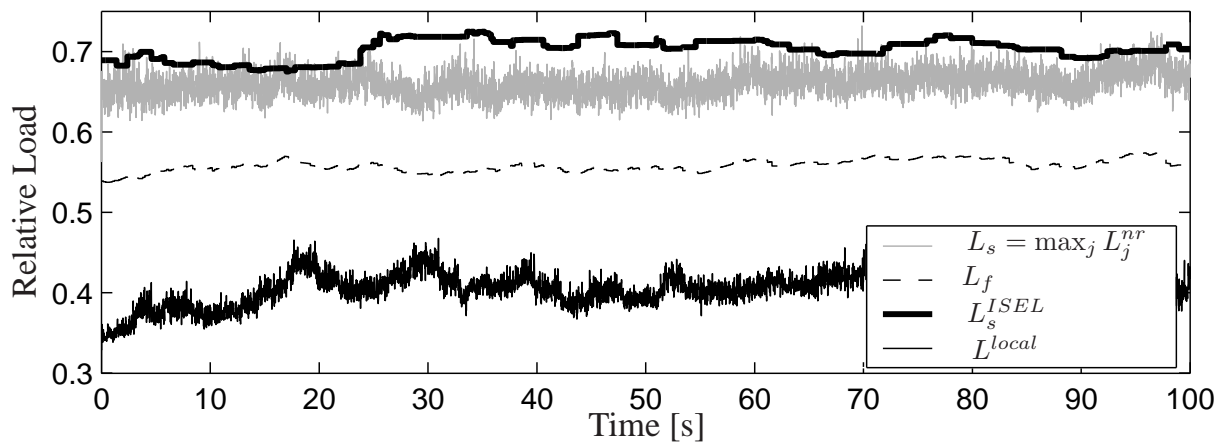

Figure 6.3: An example of the load expressions and bounds discussed in Theorem 6.5 and Theorem 6.6. $L^{\text {local }}$ is the lower bound in Theorem 6.6. Correction for $T X$ increase has been used when calculating $L_{s}^{I S E L}$.

\subsection{Summary}

In this chapter a number of alternative definitions of uplink load has been given. One of them, feasibility relative load, directly relates uplink load to existence of a solution to the power control problem. It has been shown that, in a single cell system, this definition coincides with the more traditional definition of load related to noise rise, uplink noise 
rise relative load. However, in a general system they are different. Despite this, it has been shown that expressions for uplink load derived in Chapter 5 can under somewhat idealistic circumstances be used to provide bounds on the rather theoretically oriented feasibility relative load.

All expressions for uplink noise rise in Chapter 5 originate from a system of nonlinear equations. One way of solving these equations is to use iterations. The iterations are obviously useful only if they converge to a stationary point. A number of sufficient conditions for existence of such a point was established in this chapter, and the point has been shown to be unique in case if the system has connectivity one. The analysis also showed that the convergence point is the true noise rise level in case of a feasible system with connectivity one. 


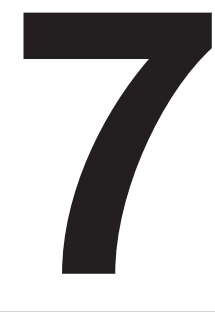

\section{Controlling Uplink Load}

Resource control is about assigning resources to the users. As such, it is a part of the system's resource management. It is a delicate task, since not only must system feasibility be maintained while maximizing the resource utilization, there is also a trade off between fairness and capacity. Moreover, assigning resources to a user does not necessarily mean that they will be used. For example, the user may not have enough transmission power to support the service or may not have any data to send.

These considerations imply that there are advantages with both centralized and decentralized resource control schemes, just as with load approximations. The reasons for this are also pretty much the same. A central node has information about the situation in a wider area and can therefore move resources from one area to another, while a local node has more accurate, detailed and up to date information on the local situation.

Partially as a motivation for the work in this chapter, the load approximations derived in Chapter 5 are used for admission control. The performance of the admission control using the proposed load approximations is compared with using an approximation based on the intercell-to-intracell-interference factor. It is argued that the proposed load approximations are easier to parameterize. For example, there is no need to adapt any parameters to variations in the radio environment characteristics which is the case when using the intercell-to-intracell-interference factor. On the other hand, the centralized load approximations cannot adapt to fast changes in the radio environment, such as the multi path fading. There is thus no way of mitigating any rapid load increase due to for example user movement. A step towards solving this problem is obviously to use decentralized resource management. Following this introductory discussion, Section 7.2 gives a brief survey over different ideas for solving the resource control problem presented in the literature.

The bulk of this chapter is in Section 7.3 and Section 7.4, where a number of resource control algorithms are introduced and compared with each other. Starting with complete knowledge of the radio environment and user requests in the entire network, a centralized resource control algorithm is introduced. This algorithm is not realistic since 
it requires far too much signaling, and is thus only meant for comparison purposes. Realistic resource control algorithms making decisions in local nodes, using less and less information about the surrounding radio environment, are then introduced.

One of the introduced reference algorithms is referred to as noise rise controlling algorithm. This is an example of a type of algorithms that is commonly found in the literature. The algorithm uses no explicit information on the radio environment, such as users' relative power gain, but instead simply studies the uplink noise rise. It is shown in Section 7.5 that this type of algorithm easily suffers from unintentional shift in resources from one base station to another, or even decreased coverage, when using inaccurate knowledge of the background noise power. The chapter is finalized with a short summary in Section 7.6.

The algorithms introduced in Section 7.3 were first presented and evaluated in (Geijer Lundin et al., 2005a). The basic idea which the algorithms are built on is filed in (Geijer Lundin and Gunnarsson).

\subsection{Practical Centralized Resource Allocation}

A number of uplink load approximations are derived and evaluated in Chapter 5. To close the loop in Figure 1.1, these approximations will in this section be used as input to a centralized admission control algorithm, Algorithm 7.1.

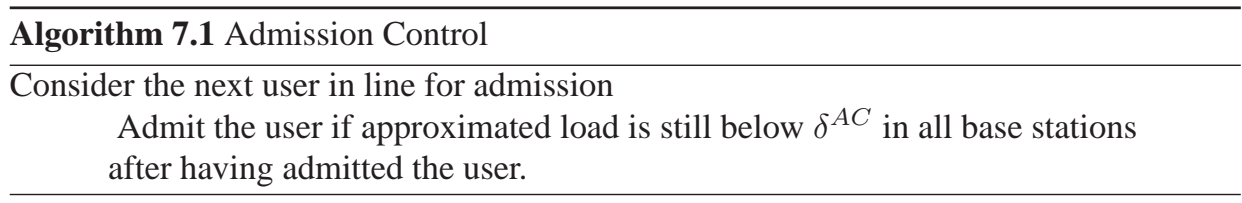

The load approximation to be used has to be able to predict the load after having admitted a user. If the predicted load in any base station is above a parameter $\delta^{A C}$, the user is not admitted.

Seven different methods for approximating the uplink load will be used. The first six of these are derived in Chapter 5, the ones referred to as $L^{I M R C}, L^{I S E L}, L^{I B O T H}$, $L^{N M R C}, L^{N S E L}$ and $L^{N B O T H}$. The seventh approximation is motivated by the discussion in Section 4.2.1 and is in cell $j$

$$
L_{j}^{r e f}=\sum_{i \in c_{j}} \frac{(1+f) \beta_{i}^{t g t}}{\left|K_{i}\right|},
$$

where $\left|K_{i}\right|$ is the number of cells that user $i$ is connected to and $f$ is the intercell-tointracell interference factor.

For increased realism, an admission control decision does not take effect until one second after the decision is made. This is to model the latency in the connection setup between user and the central node in which the load approximations as well as the admission decisions are made. A single service scenario with connectivity three offering a $384 \mathrm{kbps}$ service with target CIR of $\beta_{0}=0.14$ is studied. The simulation area is covered by 21 base stations at 7 different positions. The intercell-to-intrcell-interference factor in 
the reference approximation $L^{r e f}$ is chosen to $f=0.5$ and the load limit for admission in Algorithm 7.1 is set to $\delta^{A C}=0.8$. Further characteristics of the simulator is discussed in Appendix A.1.

Table 7.1 shows that using any of the load approximations proposed in Section 5.2.2 or Section 5.2.3 results in higher capacity per noise rise, compared to using the reference approximation. Another draw back with the reference approximation, $L^{r e f}$, is that the intercell-to-intracell-interference factor, $f$, needs to be adjusted to variations in the radio environment. The approximations proposed in Chapter 5, however, inherently considers the radio environment via the path gain measurements. There is thus no need for re-tuning the proposed approximations for different radio environments. Table 7.1 also shows that

Table 7.1: Result of admission control simulations. Target load, $\delta^{A C}=0.8$. The capacity, $C$, is according to Definition 3.2. Average over 20 simulations. $f=0.5$.

\begin{tabular}{|c|c|c|c|c|c|c|c|}
\hline & $L^{r e f}$ & $L^{I M R C}$ & $L^{I S E L}$ & $L^{I B O T H}$ & $L^{N M R C}$ & $L^{N S E L}$ & $L^{N B O T H}$ \\
\hline$C$ & 18.85 & 17.72 & 14.71 & 15.17 & 18.23 & 15.28 & 15.85 \\
\hline$L_{s}$ & 0.93 & 0.91 & 0.85 & 0.86 & 0.92 & 0.86 & 0.87 \\
\hline$C / \bar{\Lambda}$ & 1.42 & 1.65 & 2.22 & 2.14 & 1.51 & 2.10 & 2.03 \\
\hline
\end{tabular}

using any of the proposed approximations results in a slightly lower average system noise rise relative load, $L_{s}$, as defined in Section 6.2.1. The reason for this is simply that the proposed approximations consider where in the cell users are, since the users' relative power gain values are studied. The reference approximation, on the other hand, simply looks at to which cell the user is applying for a connection to, and thus do not take the resulting intercell load contribution in neighboring cells into consideration when making the admission decision. Figure 7.1 shows how the noise rise relative load progresses when using three different load approximations. The approximations studying the users' relative power gain values provide a relative load significantly closer to the load limit, $\delta^{A C}=0.8$, compared to the reference approximation.

Figure 7.2 shows an example of how noise rise evolves over time. The noise rise makes short deviations from a slow trend. The centralized approximations are rather good at approximating the slow trend, as concluded in Chapter 5, but can not catch the fast variations. Therefore, neither can a centralized resource allocation algorithm using the centralized approximations utilize the short periods of low noise rise levels. However, using decentralized resource control enables better resource utilization since it is then possible to make rapid resource allocation, perhaps through channel switching. The fast variations are primarily due to intercell interference. Gunnarsson et al. (2003); Törnqvist et al. (2004) propose a method to decrease the intercell interference by using completely decentralized resource allocation. In there, the users transmit only when the power gain to the own base station is favorable compared to a time averaged power gain. Figure 7.2 also shows that the slow trend can in fact increase almost $2 \mathrm{~dB}$ in a matter of a second. This puts a hard demand on the resource control bandwidth. If these sudden noise rise raises can be avoided, the average load can be chosen higher, i.e., using decentralized control enables smaller margin to a maximum load level due to a smaller inter-sample time.

To conclude this section, centralized resource management is good when resource 


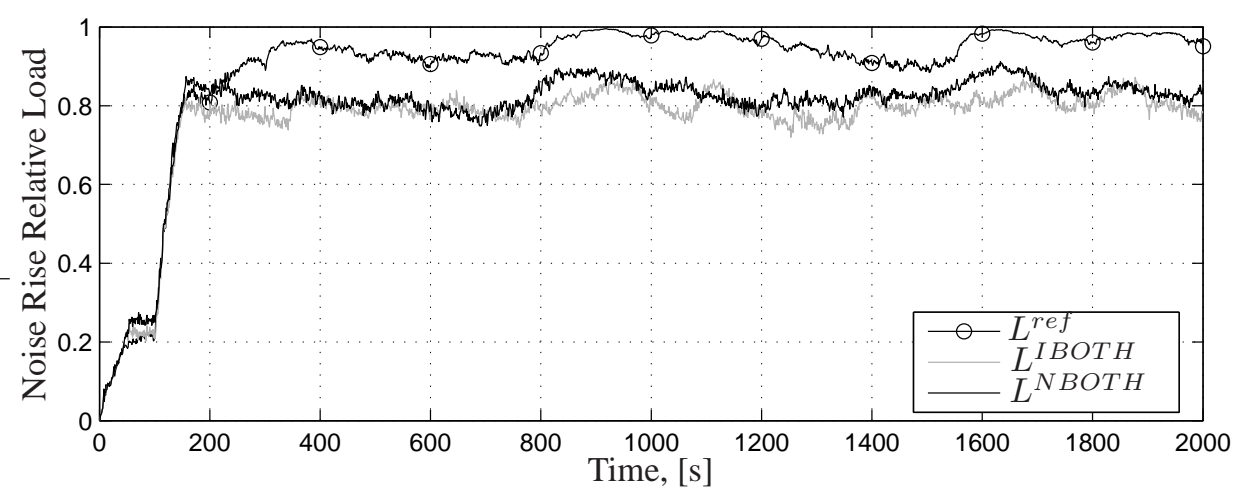

Figure 7.1: Noise rise relative load as a function of time when there is a one second delay between admission decision and transmission initialization (channel setup delay). $\delta^{A C}=0.8$.

mobility between base stations is important. However, the load margins can be chosen smaller when using decentralized resource management due to the superior control bandwidth.

\subsection{Resource Control Approaches}

Resource control is about granting users access to resources in such a manner that both the operator, who owns and operates the system, and the customers, who are users of the system, are satisfied with the performance. Typically, an operator wants as high a throughput as possible, and a user wants good coverage and high bit rates. To some extent, high bit rates are consistent with high throughput, but usually the throughput can be increased by assigning resources to only one or a few users in each cell. Even worse, increased coverage usually comes with decreased throughput. This trade off is the topic of Chapter 8.

Different views and proposed algorithms for regularly (re-)assigning resources to the users will be discussed in this section.

Optimization Approaches Cellular radio systems of today support several services. This has made the resource control problem more complex. One way of solving this complex problem is to mathematically express the quality of service (QoS) demands as a utility function and fundamental requirements such as system feasibility as constraints in an optimization problem. The QoS demands can be network-centric, for example maximizing the throughput as is done by Knopp and Humblet (1995) and Ulukus and Greenstein (2000), or user-centric as in summarizing the users' satisfaction. An example of a user-centric strategy is given by Nagi et al. (2000) where they minimize users' outage probability. Further examples of where optimization has been used to solve the resource control problem are Rodriguez et al. (2004) and Sampath et al. (1995), who study a single cell system. 


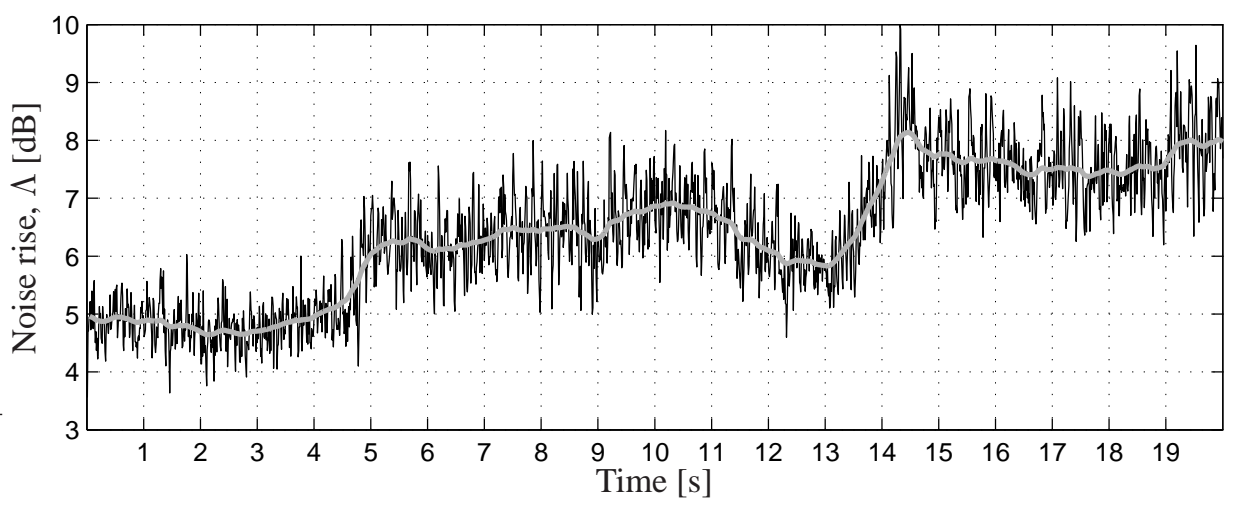

Figure 7.2: Example of noise rise. The grey plot is a time average of the the momentarily noise rise (black). The momentarily noise rise has fast variations that can both be avoided and taken advantage of by using decentralized resource control. The sudden raises in average noise rise puts hard demands on the control bandwidth of the resource control.

Power and Rate Control Power control has been recognized as a powerful tool for increasing system capacity. In fact, in CDMA cellular systems power control is crucial in order to overcome the near-far problem. However, while power control can compensate for deep fades in the channel between a user and the base station it is connected to, power control can also cause considerable intercell interference power peaks in neighboring cells. One way of getting around this problem is to consider power and rate control jointly. Instead of increasing the transmission power, the transmission rate can be decreased. Duan et al. (2002); Hashem and Sousa (1998); Kim and Lee (2000); Oh and Wasserman (2003) and Kim and Honig (2000) use this idea.

Load Control Approaches In the border line between optimization and control, Jäntti and Kim (2001) use optimal control to solve a minimum time problem. The control signals are transmission powers that each user should send within each frame. The results are derived under the assumption that the data rate is proportional to the carrier-tointerference ratio. The solution maximizes the throughput at each time instant.

Javidi (2003) solves an optimization problem using Lagrange multipliers. This leads to a control problem aiming at a certain noise rise relative load. This is a truly decentralized solution in which each base station controls a cost for the users to connect to the base station, higher cost means a smaller cell and less users.

Despite the absence of a central node, feasibility is guaranteed by Javidi (2003) through the use of a mutual agreement between the base stations. This idea is also used by Rezaiifar and Holtzman (1999) and Feng et al. (2004). A drawback with the methods given in the above references is that the local nodes require knowledge of the current received interference power, which is generally considered hard to measure accurately. 
Opportunistic scheduling The idea here is to assign resources to the user with momentarily best channel (Liu et al., 2001). This gives an unfair assignment on a short time scale. However, Berggren and Jäntti (2002) apply opportunistic scheduling in the downlink and show that it asymptotically provides the same fairness as a round robin scheduler, but with better utilization.

\subsection{Resource Control Algorithms}

As discussed in the introduction to this chapter, both centralized and decentralized resource control schemes have its advantages. For example, using local resource control algorithms enables rapid assignment of resources based on local detailed knowledge concerning for example the radio channel or users' momentarily transmission requests. When using a centralized resource control algorithm, on the other hand, it is much easier to achieve the soft capacity of the system since resources can easily be moved from one base station to another. Obviously, knowledge of the situation in several base stations can be explored in many other ways as well.

A number of resource control schemes that guarantee system feasibility are presented in this section. When studying decentralized algorithms not using a central node but yet guaranteeing system feasibility, it will be shown that system capacity can be increased by studying information about the users' relative power gain, compared to studying the corresponding power gain values. Furthermore, a resource control algorithm using both a central node and local nodes will be introduced. The idea is to take advantage of both centralized and decentralized schemes. The algorithms are presented in such an order that they have less and less information on the surrounding radio environment. Starting with a centralized algorithm and ending with the noise rise controlling algorithms using as coarse information as which base station users are connected to.

Algorithms not studying relative power gain values but instead maintain feasibility by looking at measurements of uplink noise rise will also be studied. Characteristics of this kind of algorithms is studied in Section 7.5 through a theoretical study in a simplified scenario as well as a more practical approach using simulations. As relatively high target CTIR values will be studied here, the degradation in performance due to self interference can no longer be neglected. Recalling the relation between CTIR, CIR and self interference in (3.7),

$$
\gamma=\frac{C}{I^{t o t}-(1-\alpha) C}=\frac{\beta}{1-(1-\alpha) \beta} .
$$

Furthermore, the resource control algorithms will be posed as optimization problems. The utility function in these optimization problems will often be the system capacity according to Definition 3.2. For notational ease, introduce

$$
\kappa\left(\beta^{t g t}, \alpha\right)=\sum_{i=1}^{M} \log _{2} \frac{1+\alpha \beta_{i}^{t g t}}{1-(1-\alpha) \beta_{i}^{t g t}}
$$

for the system capacity when users have target CTIR values according to the vector $\beta \in$ $\mathbb{R}^{M}$ and the self interference factor is $\alpha$. 
A final remark before introducing the resource control algorithms is that the eigenvalue corresponding to the spectral radius of a positive matrix, i.e., a matrix with only positive elements in it, is positive and real. See Appendix A.2.

\subsubsection{Centralized Robust Algorithm}

In this section a completely centralized algorithm will be introduced. The algorithm will be used as an upper bound on the capacity. As such it is provided with power gain information from the entire system.

Ideally, the users would be given target CTIR values according to the solution to

$$
\begin{aligned}
& \underset{\beta_{i}^{t g t}}{\operatorname{maximize}} \kappa\left(\beta^{t g t}, \alpha\right) \\
& \text { s.t. } L \preccurlyeq L_{t g t} E,
\end{aligned}
$$

where the utility function is the system capacity and the constraint simply states that the maximum eigenvalue of $L$ must be less than the scalar parameter $L^{t g t}$, i.e., $\bar{\lambda}(L) \leq$ $L^{t g t}<1$. This corresponds to the feasibility relative load also being less than $L^{t g t}$.

The tools used for solving the optimization problems (Löfberg, 2004; Toh et al., 2001) can only solve linear matrix inequalities (LMI) involving symmetric matrices. Therefore, the constraint in (7.1) can not be considered explicitly. However, using the Schur complement, see Appendix A.3, the LMI in (7.1) can be substituted by

$$
\left(\begin{array}{cc}
E & L \\
L^{T} & L^{t g t^{2}} E
\end{array}\right) \succeq 0 .
$$

This constraint corresponds to requiring the maximum singular value of $L$ to be less than or equal to $L^{t g t}$ squared. As the spectral radius of a matrix is always less than the square root of the maximum singular value, the constraint is harder than that in (7.1).

Substituting the constraint in (7.1) with the above LMI yields Algorithm 7.2.

Algorithm 7.2 Centralized RRA Algorithm

Assign target CTIR values according to the solution to

$$
\begin{aligned}
& \underset{\beta_{i}^{t g t}}{\operatorname{maximize}} \kappa\left(\beta^{t g t}, \alpha\right) \\
& \text { s.t. }\left(\begin{array}{cc}
E & L \\
L^{T} & L^{t g t^{2}} E
\end{array}\right) \succeq 0 .
\end{aligned}
$$

\subsubsection{Semi-Centralized Robust Algorithm}

Ways of providing more resources to some base stations and less to others include using a central node or communication between the base stations. A resource controlling algorithm using a central node as well as local nodes is proposed in this section. The central node will hand out resource pools to the local nodes hosted in the base stations. The 
algorithms in the base stations can then use these pools while respecting a mutual agreement between the base stations and the central node. The mutual agreement is crucial to guarantee system feasibility.

Resources are distributed among the base stations through the use of information feed back from the base stations to the central node. The information fed back will be about how many and where the users are located. However, due to limited bandwidth between base stations and the central node, it is not tractable to send too much information.

Figure 7.3 shows a schematic view of the information flow in the algorithm proposed here. The main idea is to limit the intercell interference by considering the relative power

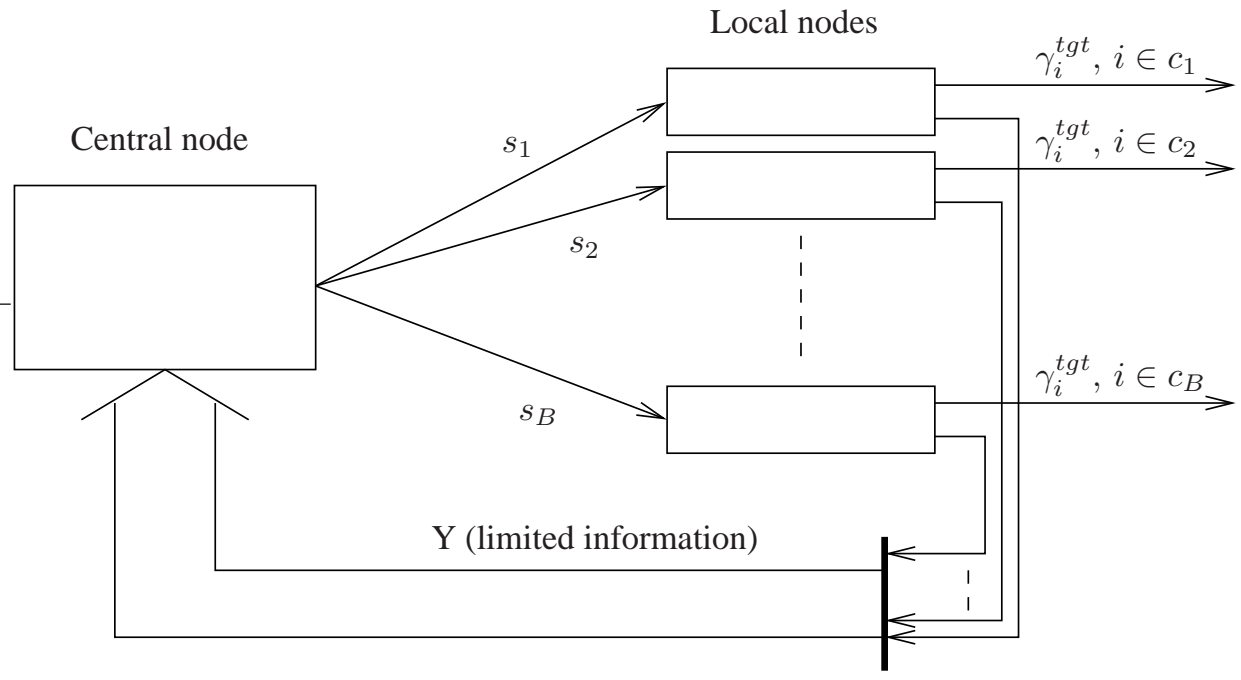

Figure 7.3: Information flow in a decentralized RRM algorithm. The central node hands out resource pools, $s_{k}$, to the local nodes. The local nodes distribute resources to the users in form of target CIR values and also provide feed back to the central node.

gains, $z_{i, j}$. The element on row $k$ and column $j$ in the system matrix $L$ is at time $t$

$$
L_{k, j}(t)=\sum_{i \in c_{k}} \beta_{i}^{t g t}(t) z_{i, j}(t)
$$

This number can be interpreted as the noise rise relative load caused by cell $k$ in cell $j$ or, more accurately put, $L_{k, j} I_{k}^{t o t}$ is the intercell interference power users in base station $k$ introduce in base station $j$. Thus, limiting the off diagonal elements of the system matrix corresponds to limiting the intercell interference. By assigning users with higher relative power gain to neighboring base stations smaller target CTIR values, each term in the elements of the system matrix can be made small without decreasing system capacity.

The resource controlling algorithm is an implementation of the lemmas that follow next. System feasibility is guaranteed by meeting the requirements in these lemmas. The 
information fed back to the central node by base station $k$ is at time $t$

$$
Y_{k, j}(t) \triangleq \frac{1}{\sum_{i \in c_{k}} \beta_{i}^{t g t}(t-1)} \sum_{i \in c_{k}} \beta_{i}^{t g t}(t-1) z_{i, j}(t-1)=\frac{1}{\sum_{i \in c_{k}} \beta_{i}^{t g t}(t-1)} L_{k, j}(t-1) .
$$

The normalizing gives a nice interpretation of the variables sent from the central node to the local nodes as resource pools.

Introduce the matrix $\bar{L}=\left[\bar{L}_{k, j}\right]$ as

$$
\bar{L}_{k, j} \triangleq s_{k}(t) Y_{k, j}(t),
$$

where $s_{k}$ is a scalar. Each $s_{k}$ will later be interpreted as a resource pool given by the central node to base station $k$, see Figure 7.3.

\section{Lemma 7.1}

If

$$
L_{k, j}(t) \leq \bar{L}_{k, j} \forall k, j \in\{1, B\},
$$

and $\bar{\lambda}(\bar{L}) \leq L_{f}^{t g t}$ then $\bar{\lambda}(L) \leq L_{f}^{t g t}$.

Proof: Follows from the fact that increasing an element of a positive matrix can not decrease its maximum eigenvalue.

The inequality in Lemma 7.1 will be used as a mutual agreement between the central node and local nodes.

\section{Lemma 7.2}

If

$$
\left(\begin{array}{cc}
E & \bar{L} \\
\bar{L}^{T} & L_{f}^{t g t^{2}} E
\end{array}\right) \succeq 0
$$

where $E$ is the identity matrix of appropriate dimension, then $\bar{\lambda}(\bar{L}) \leq L_{f}^{t g t}$.

Proof: See Appendix A.7.

Combining Lemma 7.1 and Lemma 7.2 yields the following theorem.

\section{Theorem 7.1}

A system using a resource control algorithm that meets the inequalities in (7.4) and (7.5) is feasible if $L_{f}^{t g t}$ is less than one.

Proof: As the requirement in (7.5) is met, $\bar{\lambda}(\bar{L}) \leq L^{\text {tgt }}$. According to Lemma 7.1, $\bar{\lambda}(L)$ is then also less than $L^{t g t}$ if the inequality in (7.4) is met. Finally, according to Theorem 6.2, this yields feasibility if $L^{t g t}$ is chosen less than one.

With the assumption that each $L_{k, j}(t)$ is smaller than or equal to $L_{k, j}$, the central node assigns resource pools $s_{k}(t)$ according to the solution to optimization problem (7.6). 


$$
\begin{aligned}
& \underset{s(t)}{\operatorname{maximize}} U(s(t)) \\
& \text { s.t. }\left\{\begin{array}{l}
\left(\begin{array}{cc}
E & \bar{L} \\
\bar{L}^{T} & L_{f}^{t g t^{2}} E
\end{array}\right) \succeq 0, \\
\bar{L}_{k, j}=s_{k} Y_{k, j}, k, j=1,2, \ldots, B . \\
s_{k}(t)=\sum_{i \in c_{k}} \beta_{i}^{t g t}(t) \\
\beta_{\text {min }} \leq \beta_{i}^{t g t}(t) \leq \beta_{\max }, i=1,2, \ldots, M .
\end{array}\right.
\end{aligned}
$$

As utility function, $U(s)$, one should choose a concave function as this makes (7.6) a convex problem according to Boyd and Vandenberghe (2004). A possible choice is a greedy version

$$
U_{g}(s(t)) \triangleq \sum_{k=1}^{B} s_{k}(t),
$$

which simply maximizes the combined resource pool or, in order to promote fairness for users in crowded cells, one can choose

$$
U_{f}(s(t)) \triangleq \sum_{k=1}^{B} M_{k} s_{k}(t),
$$

where $M_{k}$ is the number of users connected to base station $k$. Obviously other choices of utility function can be made. The simulations reported on in Section 7.4 use $U_{f}$.

Now, consider the local nodes. Their task is to assign target CTIR values such that the constraint in (7.4) is indeed met. By combining the resource pool $s_{k}(t)$ and the value they fed back to the central node, $Y_{k, j}(t)$, they can calculate the upper bound in (7.4) on their own. Local node $k$ will choose the target CTIR values at time $t$ according to the optimization problem in (7.9).

$$
\begin{aligned}
& \underset{\beta_{i}^{t g t}(t)}{\operatorname{maximize}} \kappa\left(\beta^{t g t}(t), \alpha\right) \\
& \text { s.t. }\left\{\begin{array}{l}
\sum_{i \in c_{k}} \beta_{i}^{t g t}(t) z_{i, j}(t) \leq Y_{k, j}(t) s_{k}(t) \forall j \\
\beta_{\text {min }} \leq \beta_{i}^{t g t}(t) \leq \beta_{\text {max }}, \forall i \in c_{k} .
\end{array}\right.
\end{aligned}
$$

Note that the requirement that the combined target CTIR values must be less than or equal to $s_{k}$ in base station $k$, is handled by the first constraint when $j=k$. If the first constraint is respected in all base stations, each element of $L$ is indeed upper bounded by the corresponding element in $\bar{L}$, i.e., the inequality in (7.4) is met. The utility function is in this case the base station capacity. Again, of course one can think of other utility functions, for example promoting fairness over capacity.

The resource control scheme introduced in this section is summarized in Algorithm 7.3. Using the iterative procedure yields that users who are not assigned any resources will not affect the values send back to the central node. Furthermore, the iterations improve the algorithm's ability to adapt to resource demands moving from one base station to another. 


\section{Algorithm 7.3 Semi-Centralized RRA Algorithm \\ 1. Given an initial matrix $Y(t)$ \\ 2. The central node assigns each base station a resource pool, $s_{k}(t), k=1,2, \ldots, B$, according to the solution to problem (7.6). \\ 3. Each base station $k$ chooses target CTIR values, $\beta_{i}^{t g t}(t) \in c_{k}$, according to the solution to problem (7.9). \\ 4. Each base station $k$ calculates new values $Y_{k, j}(t+1), j=1,2, \ldots, B$ to be send to the central node. \\ 5. Increase the time index $t, t=t+1$. \\ 6. Continue from point 2 above.}

Remark 7.1. An interpretation of $s_{k}(t)$ as a resource pool is given by studying (7.4) when Algorithm 7.3 has reached a steady state. For the special case when $k=j$, i.e., on the diagonal of $L$ (note that $z_{i, K_{i}}=1 \forall i$ ),

$$
L_{k, k}=\sum_{i \in c_{k}} \beta_{i}^{t g t} z_{i, k}=\sum_{i \in c_{k}} \beta_{i}^{t g t} \leq \bar{L}_{k, k}=s_{k} .
$$

The scalars $s_{k}(t)$ is thus an upper bound on the sum of the target CTIR values of users connected to base station $k$.

\subsubsection{Decentralized Robust Algorithms}

A result applicable to all matrices $L$ is

$$
\bar{\lambda}(L) \leq\|L\|_{\infty},
$$

where $\bar{\lambda}(L)$ is the maximum eigenvalue of $L$ (Skogestad and Postlethwaite, 1996, page 520). In the special case of positive matrices, the right hand side of the above inequality is simply the maximum row sum. Using this fact, we can prove the following theorem.

\section{Theorem 7.2 (Local Robustness)}

If users in a system with connectivity one are assigned resources in such a manner that

$$
\sum_{j=1}^{B} L_{k, j} \leq L_{f}^{t g t}, \forall k
$$

then the feasibility relative load is less than or equal to $L_{f}^{t g t}$.

Proof: For positive matrices $L \in \mathbb{R}^{B \times B}$,

$$
\bar{\lambda}(L) \leq\|L\|_{\infty}=\max _{k} \sum_{j=1}^{B} L_{k, j} .
$$


Furthermore, according to Theorem 6.2, the feasibility relative load in a system with connectivity one is $\bar{\lambda}(L)$. Hence, if all row sums of $L$ are less than or equal to $L_{f}$, so must the feasibility relative load be.

A result given by (Gantmacher, 1974, page 63) is applicable to positive matrices and states that the maximum eigenvalue is bounded from below by the minimum row sum. Hence, the upper bound in Theorem 7.2 may not be very conservative. It is even equality if all row sums equal $L_{f}^{t g t}$.

Remark 7.2. Theorem 7.2 is similar to Corollary 6.1. The only difference is that Theorem 7.2 uses row sums of the system matrix $L$ and Corollary 6.1 uses column sums. This corresponds to taking sums over the load a base stations causes and the load a base stations experiences, respectively. A result is thus that the system is feasible if either sum is less than or equal to unity.

Theorem 7.2 can be used to design a local resource control algorithm in which each base station assigns resources to the users connected to it. Since one base station controls a whole row in the system matrix, it can assign resources such that the corresponding row sum is always less than $L_{f}^{t g t}$.

The sum over elements on row $k$ in the system matrix is

$$
\sum_{j=1}^{B} L_{k, j}=\sum_{j=1}^{B} \sum_{i \in c_{k}} \beta_{i}^{t g t} z_{i, j}=\sum_{i \in c_{k}} \beta_{i}^{t g t} \sum_{j=1}^{B} z_{i, j} .
$$

An interesting observation from a practical point of view, is that if the relative power gains, $z_{i, j}$, are not already available in the local nodes all that the central node needs to provide is the relative power gain sum over base stations in (7.10). This means a considerable reduction in signaling between central and local nodes, compared to sending each users' relative power gain to the base stations.

Two different scenarios are considered in different paragraphs below. In them, bounds on the sum of users' target CTIR values are derived with and without information about the users' relative power gain values.

Not Using Relative Power Gain Information If information on the users' relative power gain to other base stations is not used, robustness can still be achieved by assuming that these relative power gains are less than some $z_{\max }{ }^{1}$,i.e.,

$$
z_{i, j}<z_{\max }, j \neq K_{i}
$$

Now, the constraint in Theorem 7.2 yields an upper bound on the sum of the users' target CTIR in each cell,

$$
\begin{array}{r}
\sum_{j=1}^{B} L_{k, j}=\sum_{i \in c_{k}} \beta_{i}^{t g t} \sum_{j=1}^{B} z_{i, j} \leq \sum_{i \in c_{k}} \beta_{i}^{t g t}\left(1+\sum_{j \neq k} z_{\text {max }}\right) \leq L_{f}^{t g t} \Rightarrow \\
s_{k}=\sum_{i \in c_{k}} \beta_{i}^{t g t} \leq \frac{L_{f}^{t g t}}{1+(B-1) z_{\text {max }}},
\end{array}
$$

\footnotetext{
${ }^{1}$ That the relative power gains to other base stations are in fact less then $z_{\max }$ has to be guaranteed by some other means in this case.
} 
where $s_{k}$ can be thought as a resource pool that base station $k$ may split arbitrarily among the users connected to it. Algorithm 7.4 explains a resource control algorithm using this idea.

\section{Algorithm 7.4 Power Gain Based Local RRA Algorithm}

Each base station $k$ assigns target CTIR values to the users in $c_{k}$ according to the solution to the following optimization problem.

$$
\begin{aligned}
& \underset{\beta_{i}^{t g t}}{\operatorname{maximize}} \kappa\left(\beta^{t g t}, \alpha\right) \\
& \text { s.t. }\left\{\begin{array}{l}
\sum_{i \in c_{k}} \beta_{i}^{t g t} \leq \frac{L_{f}^{t g t}}{1+(B-1) z_{\max }} \\
\beta_{\text {min }} \leq \beta_{i}^{t g t} \leq \beta_{\max }, \forall i \in c_{k} .
\end{array}\right.
\end{aligned}
$$

Note that the sum of users' target CTIR in the whole system is upper bounded by

$$
\sum_{j=1}^{B} s_{j} \leq B \frac{L_{f}^{t g t}}{1+(B-1) z_{\max }} .
$$

As the number of base stations, $B$, tends to infinity this upper bound tends to $L_{f}^{t g t} / z_{\max }$.

Using Relative Power Gain Information By looking at the expression in (7.10), we can conclude that users with high relative power gain to other base stations should be given smaller target CTIR values. Algorithm 7.5 uses this idea. The only difference be-

Algorithm 7.5 Relative Power Gain Based Local RRA Algorithm

Each base station $k$ assigns target CTIR values to the users in $c_{k}$ according to the solution to the following optimization problem.

$$
\begin{aligned}
& \underset{\beta_{i}^{t g t}}{\operatorname{maximize}} \kappa\left(\beta^{t g t}, \alpha\right) \\
& \text { s.t. }\left\{\begin{array}{l}
\sum_{i \in c_{k}} \beta_{i}^{t g t} \sum_{j=1}^{B} z_{i, j} \leq L_{f}^{t g t} \\
\beta_{\text {min }} \leq \beta_{i}^{t g t} \leq \beta_{\max }, \forall i \in c_{k} .
\end{array}\right.
\end{aligned}
$$

tween Algorithm 7.4 and Algorithm 7.5 is in the constraints. The feasible region of the optimization problem considered in Algorithm 7.5 is larger than that in Algorithm 7.4 since $z_{i, j} \leq z_{\max }$. The former will therefore provide a capacity at least as high as Algorithm 7.4 will. Another way of looking at it is that Algorithm 7.4 assumes that all users stand in the worst position possible, and must therefore be more conservative than Algorithm 7.5.

By looking at the constraints in (7.12), the cost for assigning resources to a user is proportional to the user's sum over relative power gain values. Therefore, as a user's contribution to the total capacity only depends on its target CTIR, the user with lowest relative power gain sum will be assigned the highest target CTIR when using Algorithm 7.5. 


\subsubsection{Blind Algorithms}

For comparison reasons, two more algorithms will be introduced. These algorithms are blind in the sense that they will not consider the users' power gain values, but merely to what base station the users are connected to.

Noise Rise Controlling Algorithm A straightforward way of implementing decentralized resource management by controlling the uplink noise rise. To exemplify, Algorithm 7.6 is an implementation of this idea. It is common to use a noise rise controlling

Algorithm 7.6 Noise Rise Controlling Algorithm

For each base station $k$ and each time step

1. Establish the uplink noise rise.

2. Assign target CTIR values, $\beta_{i}^{t g t} \in\left[\beta_{\min }, \beta_{\max }\right] \forall i \in c_{k}$ while aiming at a noise rise of $\Lambda^{t g t}$.

algorithm for local resource management, see for example the work done by Damnjanovic et al. (2002); Neubauer and Bonek (2001) or Javidi (2003). A basic requirement for using such an algorithm is obviously that the uplink noise rise can be made available through measurements of the uplink interference power or in some other way, e.g., using the approximations in Chapter 5.

While this simple idea guarantees system feasibility, it suffers from other weaknesses. One of these is that resources can be unintentionally shifted between base stations. This problem is studied in Section 7.5.

Cell Based Algorithm Algorithm 7.7 is inspired by with what is done in an addition to the uplink in WCDMA called Enhanced UplinkParkvall et al. (2005). The algorithm uses fixed resource pools, $s_{k}=s_{0}$. The constant $s_{0}$ is a design parameter which should be chosen with consideration taken to the amount of intercell interference and the trade off between system capacity and probability of assign resources corresponding to an in feasible system. Since the algorithm does not consider the radio environment more than to which base station users are connected to, it can not guarantee system feasibility. When setting the resource pool, the intercell-to-intracell-interference factor can be considered. A higher expected intercell-to-intracell-interference factor can be handled with choosing a smaller resource pool, $s_{0}$. In a sense, this algorithm is the opposite of the completely centralized algorithm, Algorithm 7.2. The algorithm is user-centric as opposed to network-centric since it assign equal resources to all users connected to the same base station. Most important, though, is that Algorithm 7.7 does in no way guarantee system feasibility. 


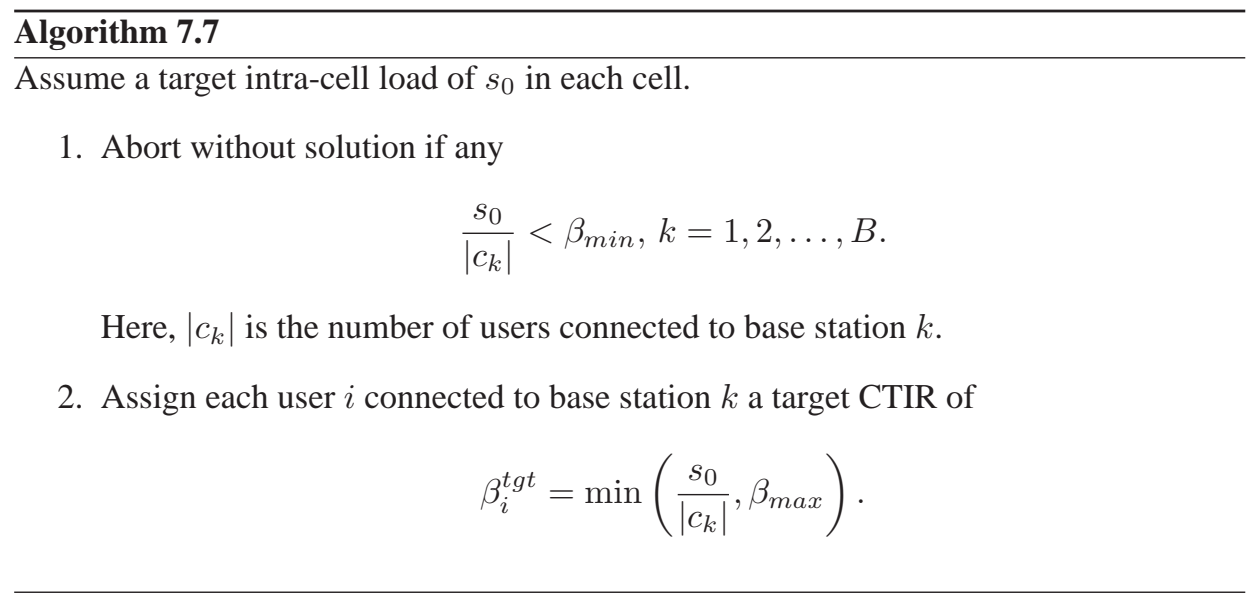

\subsection{Resource Control Evaluations}

Three of the algorithms introduced in Section 7.3 will here be evaluated and compared with a rather simple resource controlling algorithm. The algorithms from the previous sections that will be evaluated are

- Algorithm 7.2, which is a completely centralized algorithm

- Algorithm 7.3, which uses both a central node and local nodes

- Algorithm 7.5, which is a decentralized algorithm using independent local nodes

- Algorithm 7.6, which uses knowledge of the uplink noise rise

- Algorithm 7.7, which is a decentralized algorithm

The first three algorithms have in common that they all guarantee a feasible resource control if they are provided with accurate power gain measurements. Algorithm 7.6 studies the noise rise and thereby guarantee system feasibility, but all users in a cell will be assigned equal target CTIR. No attempts to minimize the intercell interference will thus be made by the algorithm. Algorithm 7.7 does not take any consideration to the intercell interference nor the noise rise level, so system feasibility is therefore not guaranteed.

Two different scenarios will be simulated. The users are spread over the simulation area using a uniform distribution in the first scenario. In the second scenario, a big part of users are concentrated to small area. This will produce high load in some base stations and less load in the surrounding ones.

The simulation area consists of 9 base stations grouped into three different positions. The cell diameter is 750 meters and the attenuation exponent is $\alpha=-3.52$. After one of the resource control algorithms has assigned target CTIR values to the users, the values are adjusted to compensate for limited transmission power. The users' maximum transmission power is $p_{\max }=21 \mathrm{dBm}$. In this simple study, only static simulations have been used, i.e., no user movement or power control has been simulated. This corresponds to assuming perfect power control and as fast a resource control that the changes in radio 
environment due to user movement can be neglected. The users' power gain values are taken from the simulator explained in Appendix A.1. Furthermore, Löfberg (2004) and Toh et al. (2001) were used to solve the optimization problems in the algorithms.

The impact of self interference can be neglected if the target CTIR can be assumed small, as Figure 3.4 suggests. As opposed to earlier work in for example Chapter 5, large target CTIR will be studied here, which is why self interference will be considered in the simulations. The self interference factor is chosen to $\alpha=0.1$.

For increased statistic accuracy, 50 Monte Carlo simulations are used for each load level. The minimum and maximum target CTIR values are chosen to $\beta_{\min }=-15 \mathrm{~dB}$ and $\beta_{\max }=0.5$, respectively.

The result of the semi-centralized algorithm, Algorithm 7.3, depends on the initial matrix $Y$. For the purpose of calculating the initial information to feed back to the central node, introduce

$$
i_{j} \triangleq \arg \min _{i \in c_{j}} \sum_{k} z_{i, k} .
$$

The initial $Y$ is calculated using (7.3) with all users target CTIR equal to $\beta_{\text {min }}$ except user $i_{j}$ in each cell $j$ who is instead given a target CTIR of $\beta_{\max }$. This choice of initial target CTIR values is influenced by the way resources are chosen by Algorithm 7.5.

Uniformly distributed users Figure 7.4a and Figure 7.4b show the system capacity and the variance of the users' target CTIR values as a function of the maximum noise rise, where

$$
\text { maximum noise rise }=\max _{j} \Lambda_{j} .
$$

It is clear from the plots that the algorithms studying the users' relative power gain perform better from a network centric perspective. However, the usual trade off between capacity and fairness, or between network and user centric objectives, is apparent when looking at the middle plot. The users' target CTIR variance is considerably smaller when using either of the reference algorithms, Algorithm 7.6 or Algorithm 7.7, indicating that these algorithms promote fairness more than the algorithms optimizing the system capacity.

All three algorithms studying the users' relative power gain provide approximately equal performance in terms of capacity and fairness. This means that Algorithm 7.5, which does not use a central node at all, provides the same capacity as the completely centralized algorithm. However, as Figure 7.4c shows, Algorithm 7.5, is not as good as the other two robust algorithms to find a feasible solution. The plot indicates that Algorithm 7.3 in fact manages to move resources around to find a feasible solution even for small values of the maximum allowed feasibility relative load. It is therefore likely that Algorithm 7.3 is better at finding a feasible solution for a fixed maximum allowed feasibility relative load as the offered load grows, compared to Algorithm 7.5.

Non-Uniformly distributed users In this scenario, the users are located at positions $\{x, y\}$ which are both distributed according to a bimodal distribution consisting of a Gaussian and a uniform distribution. In meters, the distribution is

$$
0.3 \mathcal{N}(0,300)+0.7 \mathcal{U}[-2000,2000]
$$


a)

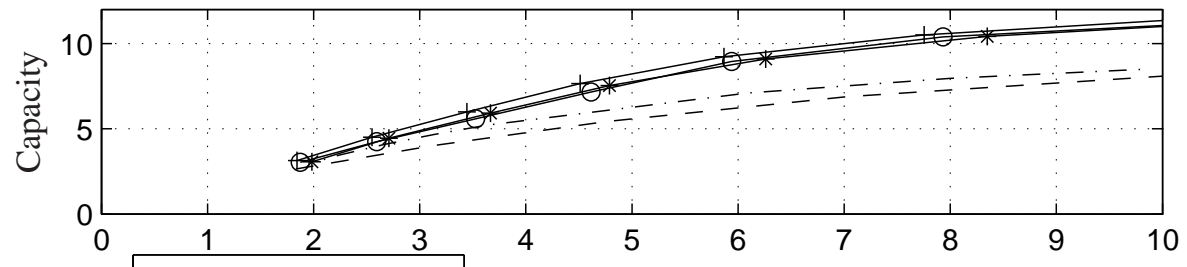

b)

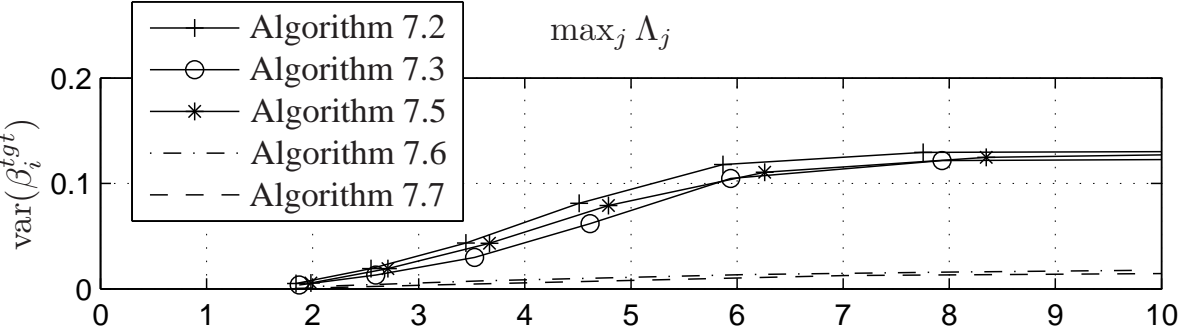

c)

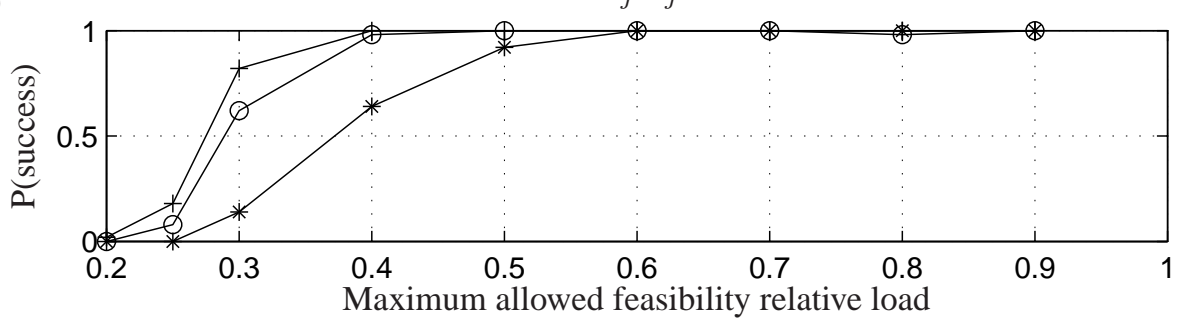

Figure 7.4: Simulation results when users are uniformly distributed. $\beta_{\min }=$ $-15 \mathrm{~dB}, \beta_{\max }=0.5, \alpha=0.1$

This gives an area with considerably higher user density. This scenario is one where there is performance to gain from moving resources from the low user density area to the high density area. This can be done by i.e., Algorithm 7.2 and Algorithm 7.3 but by the decentralized robust algorithm, Algorithm 7.5. The former two have a central node that can distribute resources between different base stations. Figure 7.5 indicates that these algorithms are indeed capable of moving resources to the highly loaded cell since, compared to the local robust algorithm, they more often find a feasible solution as shown in Figure 7.5c. Note that the Algorithms proposed in Section 7.3 and studied here, will either produce a feasible resource assignment or no result at all. This means that it is possible to in advance detect an overload situation and take necessary actions before trying to make an infeasible resource assignment leading to performance losses.

\subsection{Inaccurate Background Noise Power Knowledge}

The current section is devoted to evaluating how inaccurate information on the uplink noise rise affects the system capacity when Algorithm 7.6 is used in the base stations. In the specific scenario studied, the uplink noise rise is established by measuring the uplink interference power and dividing by the background noise power. A problem with this 
a)

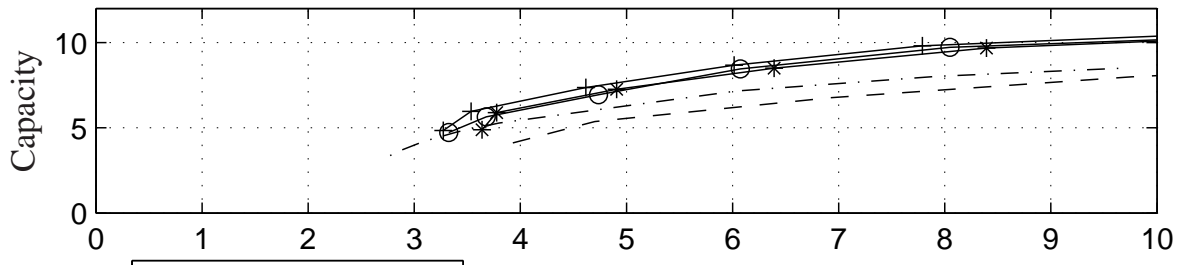

b)

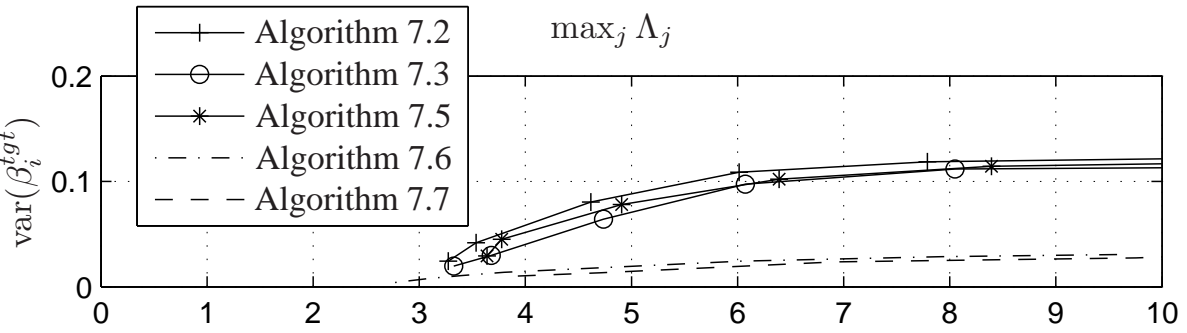

c)

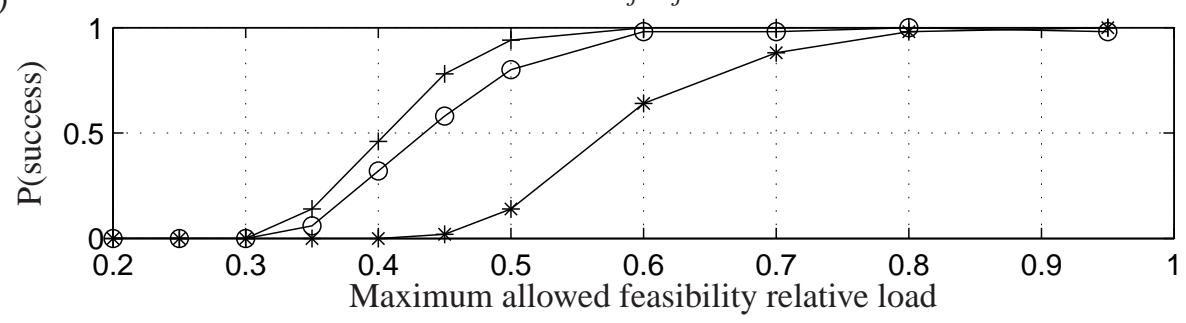

Figure 7.5: Simulation results when $30 \%$ of the users are concentrated to a small area. $\beta_{\min }=-15 \mathrm{~dB}, \beta_{\max }=0.5, \alpha=0.1$

technique is that it is hard to measure the background noise power. Inaccurate knowledge of the background noise power leads to inaccurate information about the noise rise.

Let the background noise power ratio, $\theta_{j}$, be the ratio between true and a nominal background noise power in cell $j$, i.e.,

$$
\theta_{j} \triangleq \frac{N_{j}}{N_{0}} \Rightarrow N_{j}=\theta_{j} N_{0}
$$

where $N_{0}$ is a nominal background noise power common to all cells. Using an estimate of the background noise power ratio, $\hat{\theta}_{j}$, and accurate measurements of the uplink interference power, the uplink noise rise in cell $j$ can be estimated as

$$
\hat{\Lambda}_{j}=\frac{I_{j}^{t o t}}{\hat{\theta}_{j} N_{0}}=\frac{\theta_{j}}{\hat{\theta}_{j}} \Lambda_{j} .
$$

The ratio between true and estimated noise rise is thus

$$
\rho_{j} \triangleq \frac{\Lambda_{j}}{\hat{\Lambda}_{j}}=\frac{\hat{\theta}_{j}}{\theta_{j}} .
$$




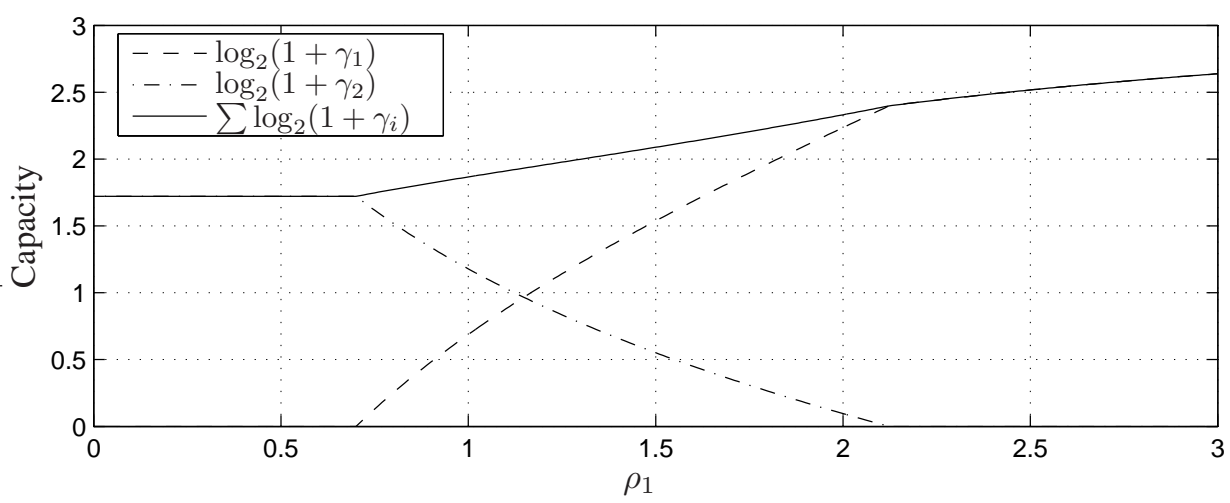

Figure 7.6: Capacity's dependence on inaccurate information on background noise power. $\Lambda^{\max }=6 \mathrm{~dB}, z_{1,2}=0.4, z_{2,1}=0.6, \alpha=0.1, \rho_{2}=1, N_{1}=N_{2}$.

The aim of this section is to study how the base station capacities change as the $\rho$ values in the different base stations deviate from one.

A $\rho_{j}<1$ corresponds to $\Lambda_{j}<\hat{\Lambda}_{j}$, i.e., the true noise rise is lower than what the resource assigning algorithm thinks. This causes a decrease in resource utilization in cell $j$. Exactly how much the capacity is decreased will be studied in two examples below.

\section{Example 7.1}

Consider a system consisting of just two base stations, or likewise, assume that two base stations can be isolated from the remaining system. For presentational ease, it is assumed that only one user at a time in each cell is transmitting, user one in cell one and user two in cell two. Consider a noise flat system with connectivity one. Solving for the uplink interference power in (6.1) and dividing by the common background noise power result in a matrix expression according to

$$
\left(\begin{array}{c}
\Lambda_{1} \\
\Lambda_{2}
\end{array}\right)=\left(\begin{array}{l}
1 \\
1
\end{array}\right)+\left(\begin{array}{cc}
\Lambda_{1} & \Lambda_{2} z_{2,1} \\
\Lambda_{1} z_{1,2} & \Lambda_{2}
\end{array}\right)\left(\begin{array}{l}
\beta_{1} \\
\beta_{2}
\end{array}\right)
$$

Now, if the local noise rise controlling algorithm in base station $j$ aims at an estimated noise rise of $\Lambda^{\max }$, the true noise rise will be $\Lambda_{j}=\Lambda^{\max } \hat{\theta}_{j} / \theta_{j}=\rho_{j} \Lambda^{\max }$. The above matrix expression can under these assumptions be written as

$$
\Lambda^{\max }\left(\begin{array}{l}
\rho_{1} \\
\rho_{2}
\end{array}\right)=\left(\begin{array}{l}
1 \\
1
\end{array}\right)+\Lambda^{\max }\left(\begin{array}{cc}
\rho_{1} & \rho_{2} z_{2,1} \\
\rho_{1} z_{1,2} & \rho_{2}
\end{array}\right)\left(\begin{array}{l}
\beta_{1} \\
\beta_{2}
\end{array}\right) .
$$

Figure 7.6 shows the capacity of the two base stations as well as the sum of the capacities as function of $\rho_{1}$ when $\rho_{2}=1$. When solving (7.14) above, the natural constraints that $0 \leq \beta_{i} \leq 1$ has been taken into account. If the solution to (7.14) implies a $\beta_{i}<0$, a single cell scenario is studied instead, i.e., the cell with $\beta_{i}<0$ will be omitted.

Naturally, the capacity in cell one increases with $\rho_{1}$, which is also apparent in Figure 7.6. This gain in capacity in cell one comes with decreased capacity in the neighboring cell. A too low $\theta_{1}$ will lead to loss in coverage since cell one is effectively not providing any service at all. For example when $\rho_{1}<0.55, \gamma_{1}=0$ because the base station thinks that the intercell interference causes a $\Lambda_{1}>\Lambda^{\max }$. Conversely, a too high $\rho_{1}$ leads to absence of service in cell two. 


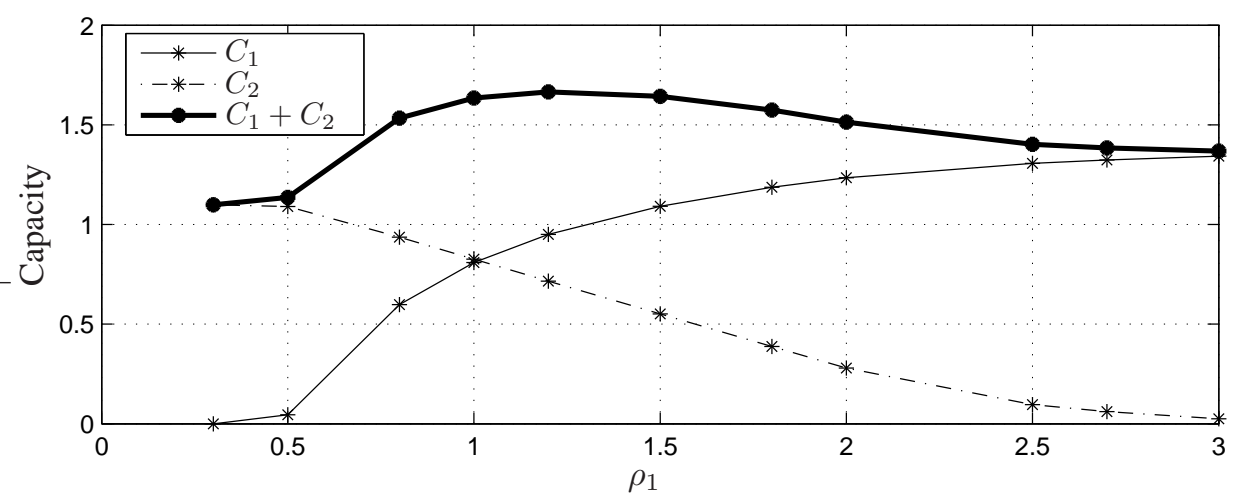

Figure 7.7: Capacity's dependence on inaccurate information on background noise power. The capacity in base station $j$ is denoted $C_{j} . \Lambda^{\max }=6 \mathrm{~dB}, \alpha=0.1, \rho_{2}=1$, $N_{1}=N_{2}$.

Behavior of the base station capacities in Example 7.1 with specific choices of relative power gain values and only one user in each cell can be generalized. Clearly, underestimating the background noise power, i.e., $\rho<1$, corresponds to a capacity loss in the own cell, but other cells experience lower intercell interference. The total system capacity may thus not decrease in the general case, but resources will definitely be transferred between cells. Conversely, overestimating the background noise power, i.e., $\rho>1$, leads to increased capacity in the own cell also since it corresponds to underestimating the noise rise yielding a true noise higher than $\Lambda^{\max }$. A significant error, such as $\rho<0.5$ corresponding to under estimating the background noise power with $3 \mathrm{~dB}$, will certainly cause an unintentional shift of resources between the base stations. Example 7.2 shows an example of how the resources are shifted when considering several users in each cell.

\section{Example 7.2}

Consider a noise flat system with two base stations. The same calculations as were done in Example 7.1 can be used to consider several users in each base station. As opposed to Example 7.1, simulations are this time used. In each of the 50 performed Monte Carlo simulations, 40 users are spread over an area between the two base stations. The path gain model in the simulator described in Appendix A.1 is used. To simplify the calculations, all users in the same base station will be assigned the same target CTIR, $s_{j}, j=1,2$.

Figure 7.7 shows how resources are transferred from base station two to base station one as $\rho_{1}$ increases. As long as $\rho_{1}$ is small, increased $\rho_{1}$ also yields higher total capacity. However, too high values of $\rho_{1}$ implies that the capacity loss in base station two is bigger than the capacity increase in base station one yielding a loss in total capacity.

The example indicates that it is important to have accurate knowledge of the background noise power, otherwise capacity is unintentionally transferred between base stations and the total capacity is decreased in general. 


\subsection{Summary}

Simulations indicate that using knowledge about the users' relative power gain values enables better resource control. Both when using centralized and decentralized algorithms. In the case of centralized control, the load approximations studied in Chapter 5 were used for admission control. By choosing users with a more favorable radio environment, higher capacity can achieved with the same noise rise compared to when using a load approximation not considering the users' radio environment.

However, due to the slow update rate and the latency in performing the resource management decisions, a completely centralized solution is not appealing in practice. Instead, a degree of decentralization should be introduced in order to better adapt and take advantage of a changing radio environment. Two algorithms that make local resource management decisions were proposed, one that uses a central node to distribute resources over different base stations and one algorithm that does not have a central node at all. A simulation study of the performance of the algorithms implied that considering the relative power gain in the resource allocation gives better performance compared to only studying which base station the users are connected to. More importantly, the algorithms using relative power gain either produce a feasible resource assignment or no assignment at all. This means that it is possible to, in advance, decide if the resource demands are too high.

A conceptual study of a group of decentralized resource control algorithms, here referred to as noise rise controlling algorithms, was introduced. These algorithm can, in a way, be seen as the opposite to the practical centralized algorithms since they only study the uplink noise rise in the own cell. The evaluation showed that neither these algorithms are well suited for practical usage due to a possible unintentional shift of resources. This shift may lead to increased capacity in a few base stations but will definitely imply decreased coverage or even lack of service in other. 


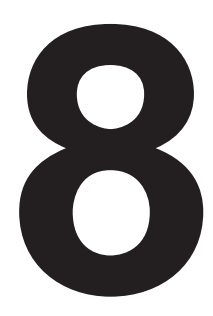

\section{Feasibility versus Coverage}

Load has always been related to system feasibility in the previous chapters. It has always been a question on whether or not the system is feasible. This chapter focuses on the load level's impact on the relation between performance measures such as coverage, capacity and users' individual service quality. This trade off is touched upon in Section 4.1, when motivating the definition of noise rise relative load. The analysis in this chapter leads to a discussion on how to set the target values of the resource control algorithm in different system deployments.

Section 8.1 studies a scenario where the users have unlimited transmission power. It is concluded that there is a trade off between signal quality and cell size in a multi cell system where the users interfere with each other, even with unlimited transmission powers. The type of limitations is also put in a system deployment perspective.

A user transmission power limitation has been applied in Section 8.2. This implies a different dependence on the users power gain distribution. A stochastic approach to link budgets is adopted. This leads to guide lines for choosing the maximum uplink noise rise relative load as a function of the background noise power in systems with large cells. Results of the link budgets are also used as input to a discussion about what quantities are important in different system deployments, when the users' transmission powers are limited.

The two different scenarios, with and without transmission power limitations, are combined in a discussion on how to choose the target load level for resource management algorithms in Section 8.3.

Results presented in this chapter are partially given in (Geijer Lundin et al., 2005b). 


\subsection{Unlimited Transmission Powers}

\subsubsection{Two Users}

Consider a single service system with two users, which are solely power controlled by base station one and two, respectively. The system matrix, as defined in Definition 6.6, is in this case simply

$$
L=\beta_{0}\left(\begin{array}{cc}
1 & z_{1,2} \\
z_{2,1} & 1
\end{array}\right),
$$

where $\beta_{0}$ is the target CTIR of the only service provided. According to Theorem 6.2 the feasibility relative load of this system is

$$
L_{f}=\beta_{0}\left(1+\sqrt{z_{1,2} z_{2,1}}\right) .
$$

We can thus conclude that a requirement for maintaining the feasibility relative load below $L_{f}$ is

$$
\sqrt{z_{1,2} z_{2,1}}+1<\frac{L_{f}}{\beta_{0}}
$$

A higher $\beta_{0}$ puts higher demands on the product $z_{1,2} z_{2,1}$. As user one moves closer to base station one, $z_{1,2}$ increases. This results in tighter demands on $z_{2,1}$, i.e., user two eventually also has to move closer to base station two. Conversely, a lower $z_{1,2}$ admits a higher relative power gain between user two and base station one.

Equation (8.1) thus shows that in order to allow for a higher target CTIR, the coverage may have to be decreased since the upper bound on the users' relative power gain is then decreased.

Remark 8.1. The relation between maximum target CTIR and the users' relative power gains is explicitly studied in the resource allocation schemes proposed in Chapter 7. Studying the relation gives two main advantages. One being that the capacity can be increased by assigning higher target CTIR values, $\beta_{i}^{t g t}$, to users who experiences favorable radio condition in terms of relative power gain. The second advantage is that system feasibility can guaranteed by the algorithms, provided that the information on the users' relative power gain values is accurate.

\subsubsection{Several Users}

In a system with several users in each cell, the system matrix is

$$
L=\beta_{0}\left(\begin{array}{cc}
\frac{M}{2} & \sum_{i \in c_{1}} z_{i, 2} \\
\sum_{i \in c_{2}} z_{i, 1} & \frac{M}{2}
\end{array}\right),
$$

where $M$ is the total number of users in the system. The assumption of equally many users in both cells is made for presentational ease. The inequality corresponding to Equation $(8.1)$ is

$$
\sqrt{\sum_{i \in c_{1}} z_{i, 1} \sum_{i \in c_{2}} z_{i, 2}}+\frac{M}{2}<\frac{L_{f}}{\beta_{0}} .
$$


The above inequality must be respected if the feasibility relative load is to be maintained below $L_{f}$. However, the constraint can be met in many different ways. For example, providing service to more users can be compensated for by decreasing the target CTIR, increasing the possible feasibility relative load or assigning service only to users with small relative power gain.

In for example systems with small cells, where the users' power gain values are relatively large, it is likely that the above inequality is in fact what limits the performance also with limited user transmission powers. The above expression not only confirms the well known fact that a dense deployment yields a capacity limited system, as opposed to coverage limited, but the expression also provides a requirement on properties directly related to coverage, capacity and quality of users' service.

\subsection{Limited Transmission Powers}

In the presence of limited user transmission power, coverage depends on the amount of received interference power at the base station antenna. This section is devoted to finding an approximative relation between the maximum received interference power and the system's grade of service when the users' transmission powers are limited.

\subsubsection{Link Budget}

Traditionally, link budgets is used to e.g., calculate a minimum power gain given constraints on received signal quality and maximum transmission power. The uplink noise rise is then represented by a constant, interference margin, which is usually chosen to approximately 3 to $4 \mathrm{~dB}$ (or, equivalently, a target load of 50 to $60 \%$ ) (Black and Wu, 2002; Laiho et al., 2002; Stevens, 1998). Herein, the link budget is used to relate GoS to average uplink noise rise given a propagation model and an approximative uplink noise rise distribution.

The total power gain between user and base station can be modeled as consisting of propagation loss, $\bar{g}^{p}$, shadow fading, $\bar{g}^{s h}$, multi path fading, $\bar{g}^{m p}$, and antenna gain and cable loss, $\bar{g}^{a c l}$. In logarithmic scale,

$$
\bar{g}=\bar{g}^{p}+\bar{g}^{s h}+\bar{g}^{m p}+\bar{g}^{a c l} .
$$

The overhead bar indicates that the quantity is in $\mathrm{dB}$. The provided service corresponds to a carrier-to-total-interference ratio (CTIR), $\bar{\beta}$, which may be expressed in logarithmic scale as

$$
\bar{\beta}=\bar{p}+\bar{g}-\bar{I}^{t o t},
$$

where $\bar{p}$ is the mobile's uplink transmission power and $\bar{I}^{\text {tot }}$ is the total received power. In logarithmic scale, the uplink total received power is

$$
\bar{I}^{t o t}=\bar{N}+\bar{\Lambda}
$$

where $\bar{N}$ is the thermal noise power plus receiver noise figure common to all users and $\bar{\Lambda}$ is the uplink noise rise. 
When the users' transmission powers are limited, it is the uplink total interference power that puts a constraint on the achievable coverage. This is realized by studying Equation (4.10), which gives that a user's transmission power is proportional to the ratio of the total interference power in the connected base station and the power gain value to the base station. Thus, higher received interference power yields higher demands on the user's transmission power. However a fixed background noise power is introduced here. This, together with Equation (8.4), enables us to discuss what the performance relations look like for different uplink noise rise levels.

Combining equations (8.2), (8.3) and (8.4) yields

$$
\begin{gathered}
\bar{\beta}=\bar{p}+\bar{g}-\bar{N}-\bar{\Lambda}= \\
\bar{p}+\bar{g}^{p}+\bar{g}^{s h}+\bar{g}^{m p}+\bar{g}^{a c l}-\bar{N}-\bar{\Lambda} .
\end{gathered}
$$

Consider a user with a target CTIR equal to $\bar{\beta}_{0}$. Equation (8.5) gives that the maximum achievable CTIR, $\bar{\beta}_{\text {max }}$, is obtained using maximum transmission power, $\bar{p}_{\text {max }}$. The GoS is the probability that $\bar{\beta}_{\max }$ is greater than or equal to $\bar{\beta}_{0}$. By using Equation (8.6) the GoS can be expressed as

$$
P\left(\bar{\beta}_{\max } \geq \bar{\beta}_{0}\right)=P\left(\bar{g}^{p}+\bar{g}^{s h}-\bar{\Lambda} \geq \bar{\beta}_{0}-\bar{p}_{\max }-\bar{g}^{m p}-\bar{g}^{a c l}+\bar{N}\right) .
$$

\subsubsection{Density Functions and Constants}

Simulations show that it is reasonable to assume that $\bar{\Lambda} \in \mathcal{N}(m, \sigma)$ (Schwartz and Yeh, 1982). On the other hand, Padovani et al. (1994) provides theoretical results showing that the interference power is no longer Gaussian in a single cell system. Instead the uplink noise rise relative load can be assumed Gaussian. Thus, as a multi cell scenario is studied here, the uplink noise rise is assumed Gaussian. The difference in noise rise characteristics also means that the results found here do not apply to a single cell scenario. The shadow fading, $\bar{g}^{s h}$, can be modeled as $\mathcal{N}\left(0, \sigma_{s h}\right)$ (Hata, 1980; Okumura et al., 1968). As both $\bar{\Lambda}$ and $\bar{g}^{s h}$ are Gaussian distributed in a multi cell scenario, so is the difference between the two. This difference will therefore be modeled as a Gaussian stochastic variable

$$
\bar{X}=\bar{g}^{s h}-\bar{\Lambda} \sim N\left(-m, \sqrt{\sigma^{2}+\sigma_{s h}^{2}}\right) .
$$

The standard deviation of the shadow fading is chosen to $\sigma_{s h}=7 \mathrm{~dB}$ in accordance with guidelines found in Laiho et al. (2002).

The propagation loss, $\bar{g}^{p}$, will be considered as a random variable. Under an assumption of uniformly distributed users, the probability density function for the distance between user and base station, $r$, is

$$
f_{r}(r)= \begin{cases}\frac{2 \pi r}{A} & \text { if } r_{\min } \leq r \leq R \\ 0 & \text { otherwise }\end{cases}
$$

where $A=\pi\left(R^{2}-r_{\text {min }}^{2}\right)$ is the area of a circular cell with a minimum distance to the base station of $r_{\min }$ and a maximum distance of $R$. According to the OkumuraHata propagation model (Hata (1980); Okumura et al. (1968)) the propagation loss in 
Table 8.1: Constants used in the link budgets.

\begin{tabular}{|c|c|}
\hline$r_{\text {min }}$ & $30 m$ \\
\hline$R$ & $1100 m$ \\
\hline$\sigma_{s h}$ & $7 \mathrm{~dB}$ \\
\hline $\bar{g}^{s h}$ & $N\left(0, \sigma_{s h}\right)$ \\
\hline $\bar{g}^{m p}$ & $-4 \mathrm{~dB}$ \\
\hline $\bar{g}^{a c l}$ & $16 \mathrm{~dB}$ \\
\hline
\end{tabular}

\begin{tabular}{|c|c|}
\hline$\Lambda$ & $N(m, \sigma)$ \\
\hline$N$ & $-103 \mathrm{dBm}$ \\
\hline $\bar{p}_{\max }$ & $26 \mathrm{dBm}$ \\
\hline $\bar{C}$ & $-31.4 \mathrm{~dB}$ \\
\hline$\alpha$ & 3.57 \\
\hline
\end{tabular}

logarithmic scale can be expressed as a function of the distance $r$ between mobile and base station,

$$
\bar{g}^{p}=\bar{C}_{p}-10 \alpha \log _{10}(r),
$$

where $\bar{C}_{p}$ and $\alpha$ are parameters of the Okumura-Hata propagation model. The probability density function for the propagation loss is the derivative of the cumulative distribution function,

$$
\begin{gathered}
\frac{d}{d \bar{g}_{0}} P\left(\bar{g}^{p}<\bar{g}_{0}\right)=\frac{d}{d \bar{g}_{0}} P\left(\bar{C}_{p}-10 \alpha \log _{10}(r)<\bar{g}_{0}\right)= \\
\frac{d}{d \bar{g}_{0}} \int_{10 \frac{\bar{C}_{p}-\bar{g}_{0}}{10 \alpha}}^{R} \frac{2 r}{R^{2}-r_{\min }^{2}} d r .
\end{gathered}
$$

This gives the probability density function for $\bar{g}^{p}$ according to

$$
f_{\bar{g}^{p}}\left(\bar{g}_{0}\right)=C 10^{-\frac{\bar{g}_{0}}{5 \alpha}}, \quad C=\frac{10^{\frac{\bar{C}_{p}}{5 \alpha}}}{5 \alpha\left(R^{2}-r_{m i n}^{2}\right)} \log (10) .
$$

In the link budget, the multipath fading, $g^{m p}$, is typically represented by a multipath fading margin. The margin is chosen to give the uplink power control enough room to maintain an acceptable CTIR despite time varying channel and uplink noise rise. The multipath fading margin is herein set to $-4 \mathrm{~dB}$. The antenna gain and cable loss combination is chosen to $\bar{g}^{a c l}=18-2 \mathrm{~dB}$. The user's maximum transmission power, $\bar{p}^{\text {max }}$, is considered fixed equal to $26 \mathrm{dBm}$ (including $2 \mathrm{~dB}$ in mobile antenna gain). Table 8.1 summarizes the parameter values used in the link budgets (Laiho et al., 2002).

\subsubsection{Simulations}

The probability density function given in (8.10) together with the Gaussian probability density function for $X$ in (8.8) yields the Grade of Service in Equation (8.7) for different $m$ and $\sigma$ according to

$$
\begin{gathered}
\mathrm{GoS}=P\left(\bar{\beta}^{\max } \geq \bar{\beta}_{0}\right)= \\
1-\frac{C}{\sigma \sqrt{2 \pi}} \int_{\bar{g}_{\min }}^{\bar{g}_{\max }} 10^{-\frac{\bar{g}}{5 \alpha}} \int_{-\infty}^{y-\bar{g}} e^{-\frac{\left(x-m_{x}\right)^{2}}{2 \sigma_{x}^{2}}} d x d \bar{g},
\end{gathered}
$$


a)

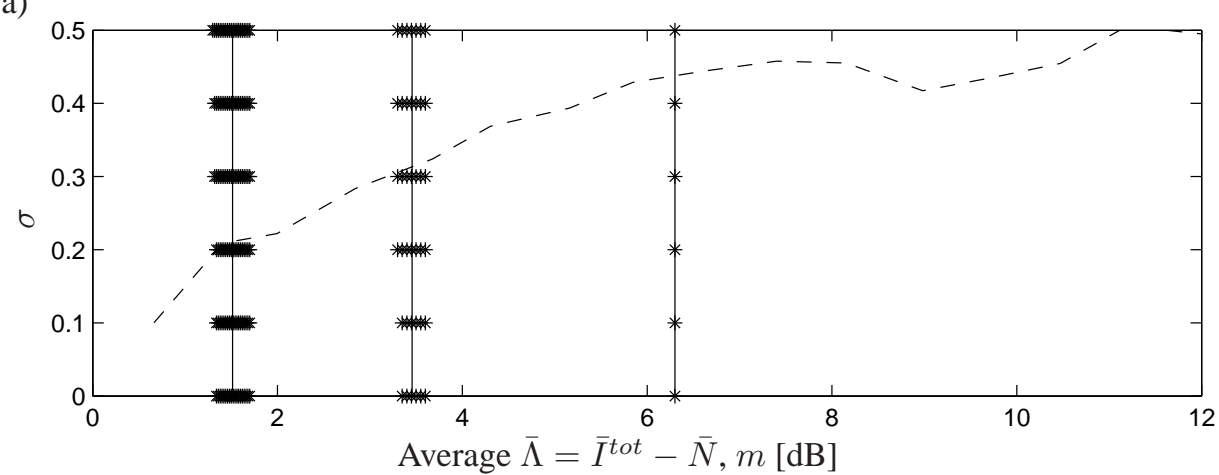

b)

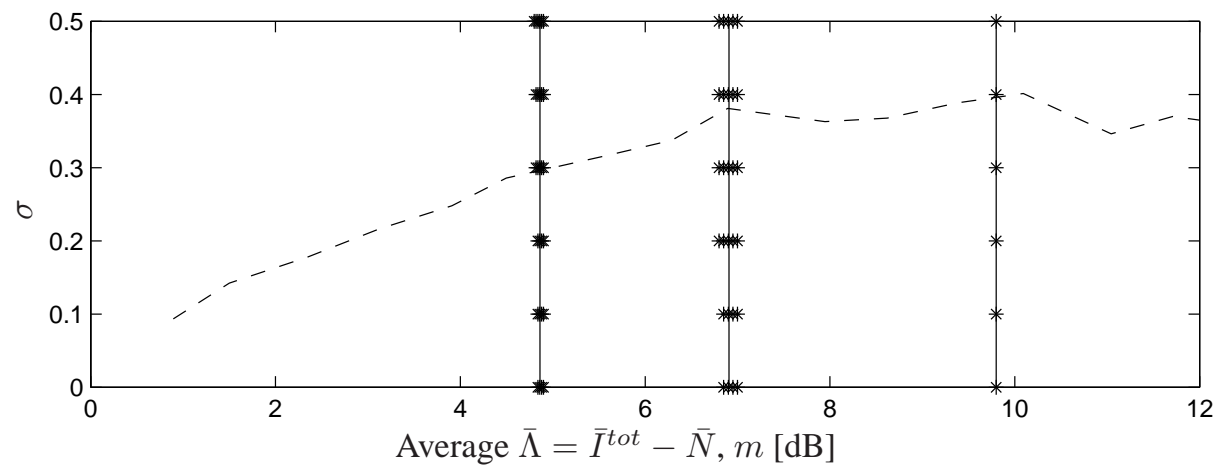

Figure 8.1: Relation between average noise rise, $m$, and standard deviation, $\sigma$, for different levels of grade of service. Also included is a plot showing the relation between average noise rise and standard deviation in simulations (dashed line). a) $\bar{\beta}_{0}=-9.5 \mathrm{~dB}$ (384 Kbps), b) $\bar{\beta}_{0}=-13 \mathrm{~dB}$ (144 kbps)

where $y=\bar{\beta}_{0}-\bar{p}_{\max }+\bar{N}-\bar{g}^{m p}-\bar{g}^{a c l}$. The integration interval for $\bar{g}$, [ $\left.\bar{g}_{\min }, \bar{g}_{\max }\right]$, is given by Equation (8.9) and the choice of $R$ and $r_{m i n}$, respectively.

\section{Grade of Service versus Maximum Noise Rise}

Figure 8.1 shows the relation between $m$ and $\sigma$ according to Equation (8.11) for two different user types and three different levels of GoS. What is important to note, even though a relation between noise rise and GoS is made here, the relation is actually one between the total received interference power and GoS. The relation between uplink noise rise and total received interference power is a one to one relation only because the background noise is chosen to a constant according to Table 8.1. As a higher background noise power yields a higher received total interference power for the same resource assignment, the background noise power indirectly affects the coverage when users have limited transmission power.

The data represented by the dashed lines is taken from a simulation of 9 cells where the only services provided were $384 \mathrm{kbps}$ or $144 \mathrm{kbps}$ services with full channel utiliza- 
tion. Even though data is from two single service scenarios, the noise rise behavior is approximately the same in a multi service scenario. The simulation results are thus applicable to a multi service scenario. The variations in noise rise are a result of imperfect power control due to, among other things, measurement errors, transmission power control errors, inter-cell interference variations and user movement. Since the lines in both plots in Figure 8.1 are rather steep, the standard deviation does not have a considerable impact on the capacity and coverage. A major reason for the independence between GoS and noise rise variance is that the shadow fading variance is considerably larger than the noise rise variance, thus $\sqrt{\sigma_{s}^{2}+\sigma^{2}} \approx \sigma_{s}$. As can also be seen in Figure 8.1, the standard deviation during simulations is quite small and also seems to be fairly service independent. From Figure 8.1 one can conclude that in order to achieve a 95 percent probability of providing service to a user assigned a 384-kbps-service with $\bar{\beta}_{0}=-9.5 \mathrm{~dB}$, the average noise rise should not be much more than $3 \mathrm{~dB}$. For a 144-kbps-service with $\bar{\beta}_{0}=-13 \mathrm{~dB}$, the corresponding noise rise figure is almost $7 \mathrm{~dB}$.

The link budgets clearly indicate a trade off between GoS and acceptable noise rise levels for a fixed background noise power. If the background noise power is increased, the maximum noise rise must decrease to the same extent. Nevertheless, the analysis shows that it is important to have accurate knowledge of the current noise rise level, or equivalently the load level, in order to guarantee a minimum grade of service. What is not as obvious from the link budgets, which considers one user at a time, is how the current noise rise level, which depends on the situation in the entire system, is established.

\section{Load versus Coverage}

The same link budget as previously used to determine a maximum noise rise level given a fixed cell radius, can also be used to determine the maximum radius as a function of the target noise rise relative load. It is simply a matter of looking at the cell radius as a variable instead of a parameter, and conversely with the average noise rise.

Figure 8.2 shows the result of such calculations when the target GoS is $95 \%$. A model stating that the average noise rise in $\mathrm{dB}$ is a linear function of the cell radius has been fitted to data. The figure shows that a large cell radius requires a low choice of maximum load level. Especially for large cell radiuses, the maximum noise rise relative load decreases fast.

The shaded areas correspond to systems that can be expected to be capacity limited, as opposed to coverage limited. These areas correspond to system deployments with cell radiuses so small that the feasibility relative load is likely to be the limiting load. Put another way, the performance for all cell radiuses is limited by the minimum requirement on noise rise relative load and feasibility relative load. In the shaded areas, it is the feasibility relative load that defines the performance limitation.

\subsection{Maximum Load}

Two different scenarios are studied in the two previous sections, with and without user transmission power limitations. Relations between capacity, coverage and users' service quality is established in a few special cases. Combining the two studies yields that the 


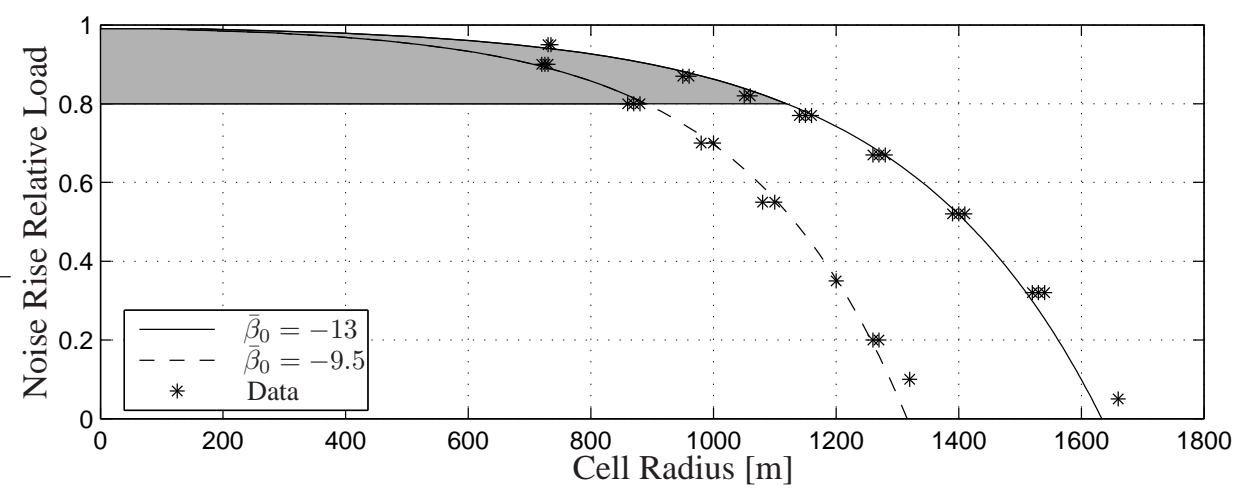

Figure 8.2: Relation between average noise rise relative load and cell radius. A simple model has also been fitted to data. GoS=95\%. The shaded area represents situations where the system is capacity limited, as opposed to coverage limited. A $\bar{\beta}_{0}$ of $-9.5 \mathrm{~dB}$ approximately corresponds to a $384 \mathrm{kbps}$ service and $\bar{\beta}_{0}=-13$ to a 144 kbps service.

trade off is apparent regardless of the transmission power limitation, but different in different scenarios.

Essentially, the system is interference limited as long as no users reach their transmission power limit. This is likely to be the case in for example a dense system, with small cells with overlap. In these system, it is the feasibility relative load that sets the reference value for the resource management algorithms in Figure 1.1.

A system with large cells is likely to instead be limited by the amount of received interference power due to the low power gain for users far from a base station. Users with low power gain to the power controlling base station become sensitive to high received interference power. Therefore, the maximum load will in this case be in terms of noise rise relative load.

Combining the results yields that the maximum uplink load depends on the specific deployment. As such, it is also possible to improve the scene by for example changing the size of some cells or applying titled antennas with lower antenna gain in suitable directions.

\subsection{Summary}

This chapter focused on how load is related to coverage and capacity in terms of users' signal qualities. The relation was studied both in presence and absence of user transmission power constraints.

Two examples were used to show that a multi cell system is in fact coverage limited, even if the users have unlimited transmission powers. The limitation is in terms of the sum of users' relative power gain values. This means that a load controlling algorithm can increase the coverage for some users at the expense of other users.

In the case when users have limited transmission power, link budgets were used to 
establish an approximative relation between grade of service, i.e., the probability of a user having service, and maximum uplink noise rise in the presence of a certain chosen background noise power. It was shown that if the maximum noise rise is chosen too high the grade of service is considerably decreased. The same link budget approach was also used to establish an approximative relation between noise rise relative load and cell radius for a certain grade of service. The cell radius also decreases rapidly with increasing load when the system is operated at high load levels.

The chapter has thus studied the well known trade off between performance measures such as system capacity, coverage and service to individual users. The overall result concerns what this trade off looks like in different deployments, and that it is very much applicable also when users do not have shortage of transmission powers. 


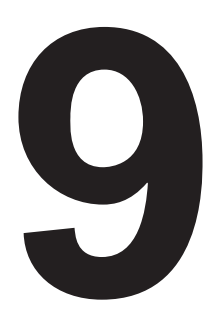

\section{Filtering and Estimating Uplink Load}

This chapter contains two signal processing application. In the first application, advanced filtering is applied to a readily available load measurement given by any of the expressions in Chapter 5. A biased auto-regressive signal model has been developed and used. Using the model together with a change detection technique results in a stable estimate which quickly adapts to new load levels. The work presented in this first part of the chapter was first published in (Geijer Lundin et al., 2003b).

The second application is estimation of the background noise power. Things get complicated due to a bias in the measurements. This makes the relation between measurements of the total received interference power on the one hand and the bias and background noise power on the other, nonlinear. It is therefore useful to apply nonlinear estimation.

\subsection{Adaptive Filtering Theory}

The filtering and estimation techniques used in later sections of this chapter are here explained. Kalman filtering is explained in Section 9.1.1. Kalman filtering was first developed for linear filtering. One way of extending it to nonlinear applications is to linearize the nonlinear model around a working point. A straight forward way is to choose a fixed working point. This idea is presented in Section 9.1.2. Another idea is to linearize about the best estimate of the current state values. This idea is used in the Extended Kalman Filter (EKF) which is explained in Section 9.1.3. The perhaps most sound idea is to not linearize at all, but instead simulate the system model as is done by the particle filters. When using particle filters, several possible state vector values are considered at the same time. In a sense, the most probable ones, based on measurements of the system model output, are kept and the rest are discarded. Section 9.1.4 discusses the particle filter method. 
A complication with applying any type of filtering or estimation is the trade off between tracking and noise suppression. This can be somewhat relaxed by studying the residuals of the estimation process. This idea and an implementation of it, the CUSUM algorithm, are explained in Section 9.1.5. A good basis for using Adaptive filtering in practice is given by Gustafsson (2000).

Introduce the notation $\hat{x}(t \mid \tau)$ for the estimate of $x(t)$ given measurements until time $\tau$. The notation is justified by the fact that at each time instant there is a measurement update, where the new measurement is considered, and a time update, where the predictions are updated.

\subsubsection{Kalman Filtering}

A basis for applying Kalman Filtering is a linear system model,

$$
\begin{aligned}
x(t+1) & =A x(t)+B_{v} w(t) \\
y(t) & =C(t) x(t)+e(t),
\end{aligned}
$$

where

$$
x(t)=\left(\begin{array}{c}
x_{1}(t) \\
x_{2}(t) \\
\cdots \\
x_{n}(t)
\end{array}\right)
$$

is the state vector containing the internal states of the model. Equation (9.1a) describes the internal dynamics of the model while (9.1b) explains how the state vector and measurement errors relate to the measurements, $y(t)$. The process noise, $w(t)$, and the measurement noise, $e(t)$, are assumed to be white Gaussian noise with zero mean. As such, they are completely characterized by their covariances $Q=\mathrm{E} w(t) w(t)^{T}$ and $R=\mathrm{E} e(t) e(t)^{T}$, respectively.

The aim is to estimate the state vector $x(t)$ in the above state space model such that the covariance of the state error, $\mathrm{E}(\hat{x}(t)-x(t))(\hat{x}(t)-x(t))^{T}$, is minimized given measurements $y(t)$. A natural way of updating the state estimate with a new measurement is

$$
\hat{x}(t \mid t)=\hat{x}(t \mid t-1)+K(t) \epsilon(t),
$$

where $\epsilon(t)=y(t)-C(t) \hat{x}(t \mid t-1)$ is called the residual at time $t$. Out of all possible ways of choosing $K(t)$, the Kalman filter (Kalman, 1960) chooses $K(t)$ such that the two-norm of $\hat{x}-x$ is minimized. This optimality holds if the noise vectors $w(t)$ and $e(t)$ are Gaussian distributed, otherwise the Kalman filter is the best possible linear filter. The Kalman gain, $K(t)$, is chosen based on knowledge of the measurement covariance, $R$, the process noise covariance, $Q$, and an estimate of the covariance of the current estimation error, $P(t \mid \tau)=\mathrm{E}(x-\hat{x}(t \mid \tau))(x-\hat{x}(t \mid \tau))^{T}$. Since $P(t \mid t)$ is time-varying it, too, has to be updated. When using the Kalman filter, the update of $P(t), K(t)$ and $\hat{x}(t \mid t)$ is done according to Algorithm 9.1. The covariance matrices $Q$ and $R$ are design parameters of the filter. Filtering $y(t)$, as opposed to predicting $y(t+1)$, corresponds to studying $\hat{x}(t \mid t)$, as opposed to $\hat{x}(t+1 \mid t)$.

To summarize, the Kalman filter delivers an estimate of the state vector $x(t)$, an estimated covariance matrix for this estimate (indicating the accuracy of the estimate), $P(t \mid t)$, and a residual $\epsilon(t)$ at each time instant $t$. 


\section{Algorithm 9.1 Kalman filter}

Initialization:

$$
\hat{x}(0 \mid-1), P(0 \mid-1)=P_{0}
$$

Measurement update:

$$
\begin{aligned}
\hat{x}(t \mid t) & =\hat{x}(t \mid t-1)+K(t)(y(t)-C(t) x(t)) \\
P(t \mid t) & =P(t \mid t-1)-K(t) C(t) P(t \mid t-1)
\end{aligned}
$$

where

$$
K(t)=P(t \mid t-1) C(t)^{T}\left(C(t) P(t \mid t-1) C(t)^{T}+R\right)^{-1}
$$

Time update:

$$
\begin{aligned}
\hat{x}(t+1 \mid t) & =A \hat{x}(t \mid t) \\
P(t+1 \mid t) & =A P(t \mid t) A^{T}+B_{v} Q B_{v}^{T}
\end{aligned}
$$

\subsubsection{Linearized Kalman Filtering}

The Kalman filter assumes that the system model is linear, both in the dynamics and in the relation between state vector and measured quantity. If this is not the case, the nonlinear model can be approximated by a linear model. To linearize the model, a working point about which the model will be linearized must be chosen. Denote the chosen working point by $x_{0}$.

Consider a system with linear dynamics, i.e., the time update of the model is linear. The nonlinearity of the model is in the measurement equation. Thus, the studied model is of the type

$$
\begin{aligned}
x(t+1) & =A x(t)+B_{v} w(t) \\
y(t) & =h(x(t), t)+e(t) .
\end{aligned}
$$

In general, the dynamics of the model can be also be time-varying, i.e., the matrices $A$ and $B_{v}$ can depend on the time index $t$. However, (9.2) suffices for our needs. Once again, the process noise $w(t)$ is independent white noise with zero mean and covariance matrix $Q$, and each measurement noise $e_{j}(t), j=1,2, \ldots, B$ is zero mean Gaussian distributed with covariance $R$.

Using a Taylor expansion about an assumed trajectory $x_{0}(t), h(x(t), t)$ may be approximated as

$$
h(x(t), t) \approx h\left(x_{0}(t), t\right)+H(t)\left(x(t)-x_{0}(t)\right),
$$

where

$$
\left.H(t) \triangleq \nabla_{x} h(x(t), t)\right|_{x=x_{0}(t)}
$$

When using the linearized Kalman filter (LKF), Algorithm 9.1 is used to estimate

$$
\tilde{x}(t) \triangleq x(t)-x_{0}(t)
$$




\subsubsection{Extended Kalman Filtering}

The extended Kalman filter (EKF) is another algorithm for estimating the internal states of the nonlinear model (9.2). EKF was first derived by Smith et al. (1962) and later by Schmidt (1966). A nice presentation of the filter based on the traditional Kalman filter is given by Kailath (1980). A system with dynamics and additive noise is studied in the above references, but the presentation here is limited to nonlinearities in the measurement equation only. The basic idea used in the EKF, is to always use a model linearized about the last estimate of the state variables. This simply means that $x_{0}(t)$ in the presentation of the linearized Kalman filter will be chosen as

$$
x_{0}(t)=\hat{x}(t \mid t-1),
$$

at time $t$.

The Kalman filter requires a linear relation between the states $x(t)$ and the measurements. Approximating $h(x(t), t)$ in (9.2) with a first order Taylor expansion around the time-varying $x_{0}(t)$ yields

$$
h(x(t), t) \approx h(\hat{x}(t \mid t-1), t)+H(t)(x(t)-\hat{x}(t \mid t-1)),
$$

where

$$
H(t)=\left.\nabla_{x} h(x(t), t)\right|_{x=\hat{x}(t \mid t-1)} .
$$

Consider a new system that has

$$
\tilde{y}(t) \triangleq y(t)-h(\hat{x}(t \mid t-1), t)+H(t) \hat{x}(t \mid t-1)=H(t) x(t)+e(t)
$$

as measurement equation. This new system has a linear relation between the measurement $\tilde{y}(t)$ and the state vector $x(t)$. Applying the Kalman filter to this new system yields the EKF applied to the original system in (9.2).

The measurement update of the EKF when applied to the model in (9.2) is

$$
\begin{aligned}
& \hat{x}(t \mid t) \\
& \quad=\hat{x}(t \mid t-1)+K(t)(\tilde{y}(t)-H(t) \hat{x}(t \mid t-1)) \\
& \quad=\hat{x}(t \mid t-1)+K(t)(y(t)-h(\hat{x}(t \mid t-1), t)+\overbrace{H(t) \hat{x}(t \mid t-1)-H(t) \hat{x}(t \mid t-1)}^{0}) \\
& \quad=\hat{x}(t \mid t-1)+K(t)(y(t)-h(\hat{x}(t \mid t-1), t)) .
\end{aligned}
$$

This and the remaining steps taken by the extended Kalman filter at each time instant are described by Algorithm 9.2.

\subsubsection{Particle Filtering}

Estimation can be seen as using noisy measurements together with prior knowledge to calculate the most probable value of the state vector. Therefore, a Bayesian approach is natural. In the Bayesian estimation formulation the time update and the measurement update are expressed as probability density functions (pdf). The idea in particle filters is to use numerical integration to express how the density functions for the values in the state 


\section{Algorithm 9.2 Extended Kalman Filter}

\section{Initialization:}

$$
P(0)=P_{0} \text { and } \hat{x}(0 \mid-1)
$$

Measurement update:

$$
\begin{aligned}
\hat{x}(t \mid t) & =\hat{x}(t \mid t-1)+K(t) \epsilon(t) \\
P(t \mid t) & =P(t \mid t-1)-K(t) H(t) P(t \mid t-1)
\end{aligned}
$$

where

$$
\begin{aligned}
K(t) & =P(t \mid t-1) H^{T}(t)\left(H(t) P(t \mid t-1) H(t)^{T}+R\right)^{-1} \\
H(t) & =\left.\nabla_{x} h(x(t), t)\right|_{x=\hat{x}(t \mid t-1)} \\
\epsilon(t) & =y(t)-h(\hat{x}(t \mid t-1))
\end{aligned}
$$

Time update:

$$
\begin{aligned}
\hat{x}(t+1 \mid t) & =A \hat{x}(t \mid t) \\
P(t+1 \mid t) & =A P(t \mid t) A^{T}+B_{v} Q B_{v}^{T}
\end{aligned}
$$

vector change as time evolves and new measurements are made. These density functions are then used to make an estimate of the values in the state vector. Because Monte Carlo integration is used, particle filters are rather computationally intensive.

The presentation here will only be on the implementation of the particle filter. Ristic et al. (2004) gives a comprehensive presentation of Monte Carlo integration and how it is used in particle filters.

It is called particle filters because several realizations, particles, of the state vector are simulated simultaneously. It is these particles that are meant to characterize the pdf of the elements in the state vector. Algorithm 9.3 specifies the particle filter. Almost arbitrarily density functions can be used to describe the measurement and process noise, not just Gaussian. However, the measurement noise must be additive. Another strength of particle filter is that there is no need to linearize the measurement equation nor any nonlinear dynamics.

\subsubsection{Change Detection}

An always present problem with traditional estimation is the trade off between tracking and noise suppression. A way of getting around this problem is to study the residuals, $\epsilon(t)$, of a process estimating the state vector of a system model. In the model used here, the residuals are expected to be zero mean Gaussian with variance $R$, i.e., $\epsilon(t) \in \mathcal{N}(0, R)$. If the estimates of $e(t), \epsilon(t)$, are not zero-mean Gaussian or have a variance which is considerably greater than $R$, there is reason to believe there has been an abrupt change in one or several of the parameters.

In order to detect a bias in the residuals, the squared normalized residuals (normalized 


\section{Algorithm 9.3 Particle Filter}

Initialization:

Initialize the particles, $\hat{x}_{i}(0 \mid-1)$, such that they cover the true state vector.

Measurement update:

- Calculate residuals for each particle $i$ :

$$
\epsilon_{i}(t)=y(t)-h\left(\hat{x}_{i}(t \mid t-1), t\right), i=1,2, \ldots, N .
$$

- Calculate the probability for each residual, $p_{i}=P_{e}\left(\epsilon_{i}\right)$ using the pdf of the measurement noise.

- Normalize the probabilities: $\bar{p}_{i}=\frac{p_{i}}{\sum_{i=1}^{N} p_{i}}$.

Resampling:

Sample particles with replacement according to the discrete probability distribution given by $\tilde{p}_{i}, i=1,2, \ldots, N$.

Time update:

$$
\hat{x}_{i}(t+1 \mid t)=A \hat{x}_{i}(t \mid t)+w_{i}(t), i=1,2, \ldots, N
$$

where $w_{i}(t)$ is drawn from the process noise's pdf.

with their estimated standard deviation), $\bar{\epsilon}^{2}$, are fed to a distance measurement algorithm. At every time instant this algorithm adds the normalized residual minus a drift term $\nu$ to the previous accumulated distance. Hence, several consecutive residuals with considerable large magnitude will result in a growing distance $g(t)$, and eventually a change detection once $g(t)$ has exceeded a fix threshold, $h$. The cumulative sum (CUSUM) algorithm (Gustafsson, 2000) is defined by Algorithm 9.4 which is run through at each update of the state vector estimate. Once a change is detected, the filter characteristics is some-

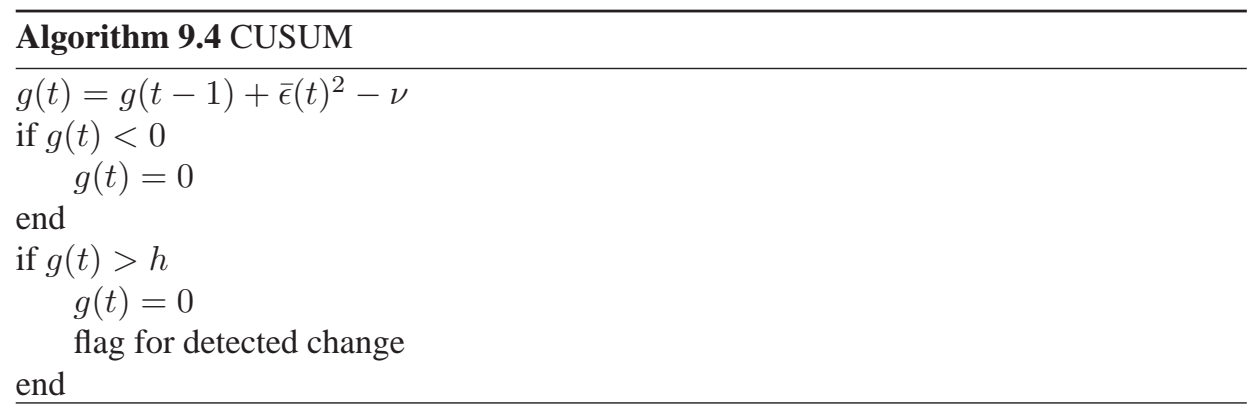

how temporarily changed in order to quickly adapt to the new situation. Figure 9.1 shows the different parts of the adaptive filter applied in this chapter and how the behaviour of the algorithms are decided by a number of design parameters. 


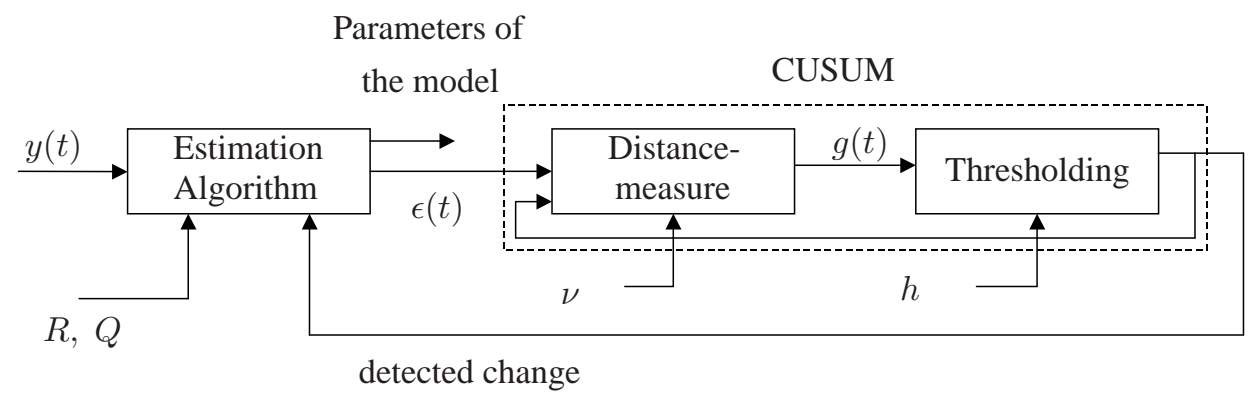

Figure 9.1: The different parts and parameters of an adaptive filter with change detection.

\subsection{Load Filtering}

The approximations given in Chapter 5 produce results which will fluctuate over time. As discussed in Section 9.2.1, the goal with the work in this section is primarily to provide a more stable approximation. In order to suppress the fluctuations, a signal model describing the fluctuations as well as the load level which the approximations fluctuate about is developed in Section 9.2.2. The filtering involves using Kalman filter and a CUSUM detector. The choice of parameter values for these algorithms are discussed in Section 9.2.3. Finally, adaptive filtering is applied to the model in Section 9.2.4.

Outcomes of the uplink noise rise relative load approximations in Chapter 5 will in this chapter be considered as measurements fed to the filtering process. They will therefore be referred to as measurements of the uplink load. They should not be mistaken for load approximations based on measurements of the uplink interference power.

\subsubsection{Motivation}

As can be seen in Figure 9.2, the raw measurement of the noise rise relative load has, just as the true load, a trend and low frequency oscillations on top of this trend. The oscillations are due to the user movement; as the users move around in the environment their number of soft handover links regularly changes. Figure 9.3 shows that oscillations in a cell's uplink noise rise are strongly correlated with oscillations in the number of soft handover links. These oscillations can easily be canceled by low pass filtering the signal. However, applying a simple low pass filter could be risky since it is important to be alert on sudden changes in the load. Therefore, a signal model together with Kalman filtering and change detection is applied to the noise rise relative load estimate in order to reduce noise and oscillations while keeping track of sudden changes in the signal. A more stable signal enables operation at a higher load level since the resource management algorithms would not need as large margin to secure stability of the system. The time-varying model also provides an indication of towards where the load is currently heading, something which enables more aggressive resource management algorithms, e.g., PID-control. 


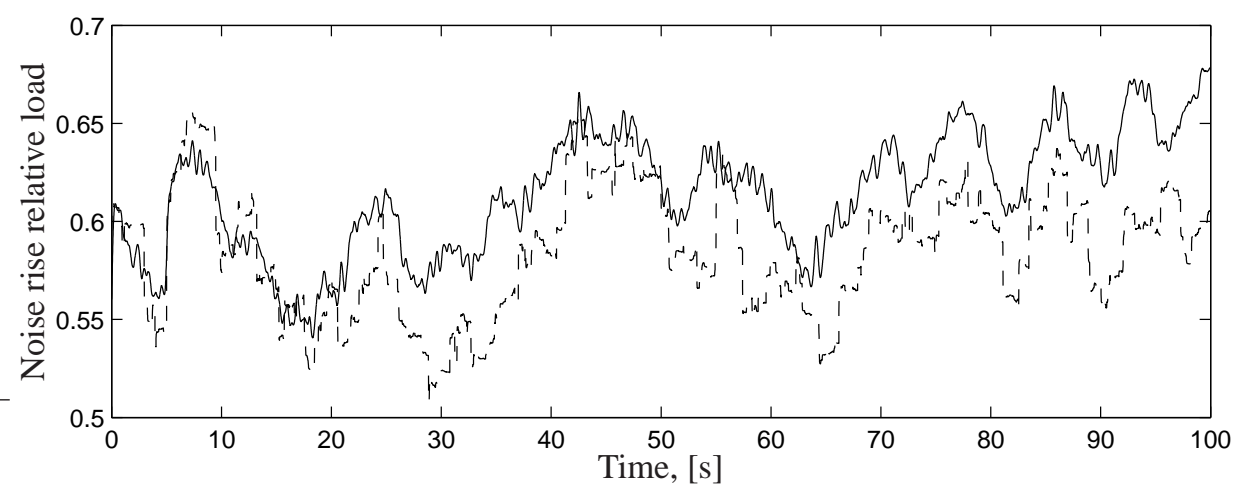

Figure 9.2: Low frequency oscillations in relative load. Solid: Low pass filtered true noise rise relative load. Dashed: Approximative noise rise relative load according to $(5.13 \mathrm{c})$.

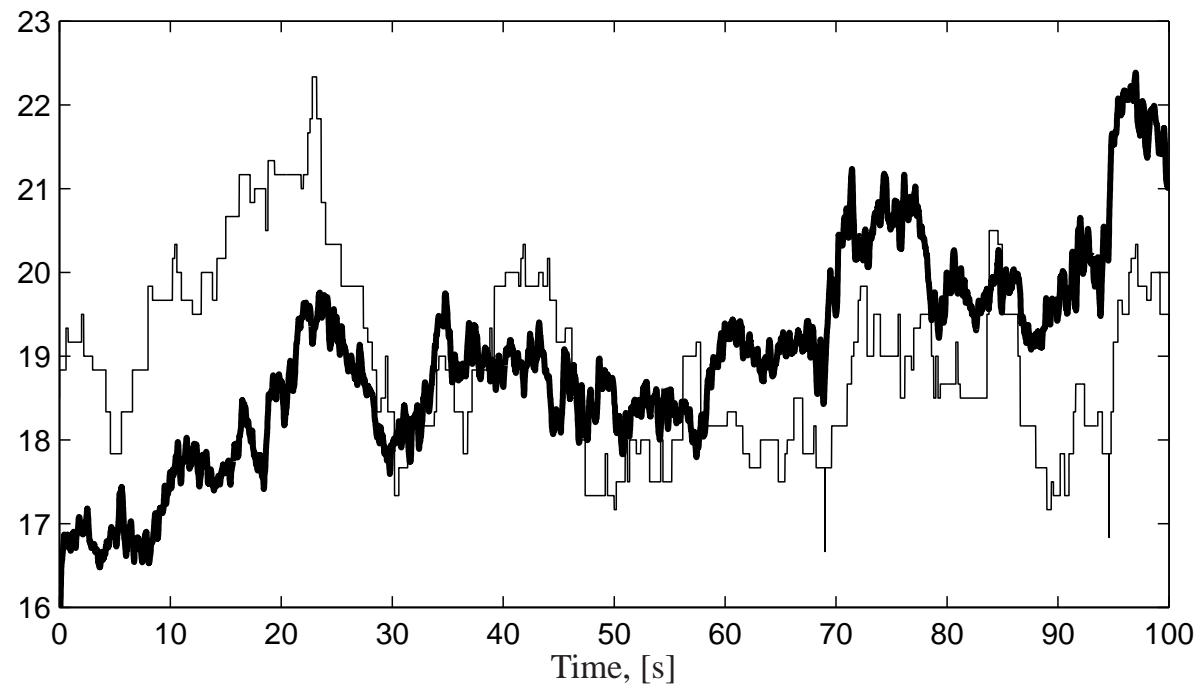

Figure 9.3: Oscillations in uplink noise rise are strongly correlated with the number of soft handover links. Thick: scaled and low pass filtered noise rise, thin: number of soft handover links 


\subsubsection{Signal Model}

When dealing with signals whose behaviour is expected to change, continuously or abruptly, a fixed model is obviously not attractive. Using a parameterized signal model and estimating not only the actual signal value but also the parameters of the model enables a more accurate signal estimate as well as an earlier detection of an abrupt change, in the parameters and/or the signal level. A signal $y(t)$, originating from an unbiased $n$ :th order Auto Regressive (AR)-model, may be described as (Ljung, 1999)

$$
y(t)+a_{1} y(t-1)+a_{2} y(t-2)+\ldots+a_{n} y(t-n)=e(t)
$$

where $e$ is white Gaussian noise with zero mean and $a_{1}, a_{2}, \ldots, a_{n}$ are the parameters of the model. The signal $y(t)$ in (9.3) will have $\mathrm{E} y(t)=0$, but the signals we study have $\mathrm{E} y(t)=\bar{L}(t)$, where $\bar{L}(t)$ is a time-varying bias. Therefore, a slightly more complex model is used, in which the time-varying bias is subtracted from each $y(t)$ giving $\mathrm{E}(y(t)-$ $\bar{L}(t))=0$. Replacing $y(t)$ by $y(t)-\bar{L}(t)$ in (9.3) yields

$$
(y(t)-\bar{L}(t))+\sum_{i=1}^{n} a_{i}(y(t-i)-\bar{L}(t-i))=e(t) .
$$

This equation defines what can be described as a biased AR model, the oscillations are no longer restricted to be around zero. There are other alternatives when modeling a signal with oscillations around a non zero mean. Alasti and Farvardin (2000) use non-zero-mean Gaussian noise to derive an expression for a biased AR model which is used to model the amount of requested bandwidth in a wireless network. A fourth order biased AR model is used to describe $y$, i.e., $n=4$ in (9.4). It is natural to assume different time scales of the bias variation and the dynamics, otherwise the AR-model is ambiguous. That is, the AR-model takes care of short term oscillations and $L(t)$ models the long term drifts. Equation (9.4) may, under these assumptions, be approximated with

$$
y(t)=\bar{L}(t)\left(1+\sum_{i=1}^{4} a_{i}\right)-\sum_{i=1}^{4} a_{i} y(t-i)+e(t) .
$$

A state space representation of (9.5) in discrete time is

$$
\begin{aligned}
& x(t)=\left(\bar{L}(t) \dot{\bar{L}}(t) a_{1} a_{2} a_{3} a_{4}\right)^{T} \\
& x(t+1)=\left(\begin{array}{ccc}
1 & T & 0 \\
0 & 1 & 0 \\
0 & 0 & E_{4}
\end{array}\right) x(t)+\left(\begin{array}{cc}
\frac{T^{2}}{2} & 0 \\
T & 0 \\
0 & T E_{4}
\end{array}\right) w(t) \\
& y(t)=\left(1+\sum a_{i} \quad 0 \quad-y(t-1) \cdots-y(t-4)\right) x+e(t) \text {, }
\end{aligned}
$$

where $w(t)$ is a 5 -dimensional vector containing the process noise and $E_{4}$ is the $4 \times 4$ identity matrix. Note that the measurement $y(t)$ has a non-linear relation to the parameters. This makes the parameter estimation more difficult. By introducing two new variables, $\tilde{L}=\left(1+\sum a_{i}\right) \bar{L}$ and $\dot{\tilde{L}}$, the nonlinear state space model is converted into a linear state 
space model according to

$$
\begin{aligned}
\tilde{x}(t) & =\left(\tilde{L}(t) \dot{\tilde{L}}(t) a_{1} a_{2} a_{3} a_{4}\right)^{T} \\
\tilde{x}(t+1) & =\left(\begin{array}{ccc}
1 & T & 0 \\
0 & 1 & 0 \\
0 & 0 & E_{4}
\end{array}\right) \tilde{x}(t)+\left(\begin{array}{cc}
\frac{T^{2}}{2} & 0 \\
T & 0 \\
0 & T E_{4}
\end{array}\right) w(t) \\
y(t) & =\left(\begin{array}{ccc}
1 & 0 & -y(t-1) \cdots-y(t-4)) \tilde{x}(t)+e(t) .
\end{array}\right.
\end{aligned}
$$

Even though $\dot{\tilde{L}}(t)$ includes time derivatives of $a_{i}$, the variation in the bias can perhaps be assumed far larger in magnitude than the variations in the AR-parameters and thus $\dot{\tilde{L}}(t) \approx\left(1+\sum a_{i}\right) \dot{\bar{L}}$. Assume the parameters to be independent of each other. This results in a diagonal covariance matrix for the process noise, $Q$, with elements $q_{1}, q_{2} \cdots q_{5}$ in the diagonal. The values $q_{2}$ to $q_{5}$ are all the same and much less than $q_{1}$. Hence we encourage changes in $\tilde{L}$ and $\dot{\tilde{L}}$ rather than in the AR-parameters. The innovations, $e(t)$ are assumed to be zero mean Gaussian with constant variance $R$.

The conversion from $\tilde{L}$ back to $\bar{L}$ is

$$
\begin{gathered}
\hat{\bar{L}}(t)=\frac{\hat{\tilde{L}}(t)}{1+\sum_{i=1}^{4} \hat{\tilde{a}}_{i}(t)}=\frac{\hat{\tilde{x}}_{1}(t)}{1+\sum_{k=3}^{6} \hat{\tilde{x}}_{k}(t)} . \\
\operatorname{Var} \hat{\bar{L}}(t) \approx L_{\hat{\bar{x}}}^{\prime}(t)^{T} \operatorname{Cov} \hat{\tilde{x}}(t) L_{\hat{\hat{x}}}^{\prime}(t) .
\end{gathered}
$$

The expression for the variance is motivated by studying a first order Taylor expansion of $\hat{\bar{L}}(t)$.

\subsubsection{Design Choices}

Figure 9.4 shows an example of a raw signal produced by the load estimation (i.e., (5.16c) and (4.3)). The period of the slowest oscillations is about 20 seconds. The sampling time of this signal is $T_{\text {frame }}=0.01$ seconds. Thus the slowest oscillations have a period of about 2000 samples. To describe the correlation in a signal having oscillations with such a long period would require thousands of parameters. A rule of thumb is that the fundamental period of the oscillations to be modeled should be approximately 10 samples (Ljung, 1999). Therefore every two hundredth sample is used when constructing the regression vector, $C(t)$. This is a kind of down sampling which requires an anti alias filter to be applied. The chosen low pass filter is a second order filter with both its poles on the real axis,

$$
y(t)=\frac{4 \cdot 10^{-4}}{1-1.96 q^{-1}+0.9604 q^{-2}} L(t) .
$$

This results in a filter which does not introduce any additional oscillations but on the other hand does not have a linear phase shift. Since it is the low frequencies of the signal that are studied here, a linear phase shift is not a requirement here. The choice of filter bandwidth is a trade off between low alias effects and small time delay between input and output of the anti-alias filter. 


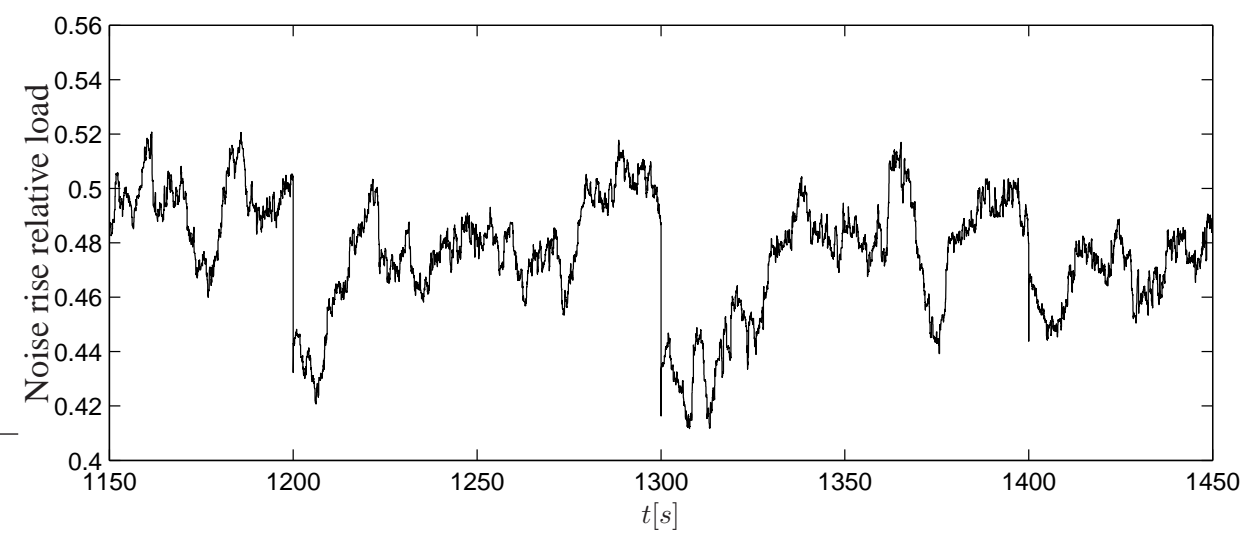

Figure 9.4: Example of noise rise relative load as function of time.

The state space representation in (9.6) has to be slightly modified to adapt to the resampling. Thus the final state space model used in the simulations is

$$
\begin{aligned}
& \tilde{x}(t)=\left(\tilde{L}(t) \dot{\tilde{L}}(t) a_{1} a_{2} a_{3} a_{4}\right)^{T} \\
& \tilde{x}(t+1)=\left(\begin{array}{ccc}
1 & T & 0 \\
0 & 1 & 0 \\
0 & 0 & E_{4}
\end{array}\right) \tilde{x}(t)+\left(\begin{array}{cc}
\frac{T^{2}}{2} & 0 \\
T & 0 \\
0 & T E_{4}
\end{array}\right) w \\
& y(t)=\left(\begin{array}{lllll}
1 & 0 & -y(t-200) & \cdots & -y(t-800)
\end{array}\right) \tilde{x}(t)+e(t) .
\end{aligned}
$$

Note that $y(t)$ is the output from the anti-alias filter, and $T$ is the update rate of the filter times the length of a frame, $T_{\text {frame }}=0.01 \mathrm{~s}$. The Kalman filter is parameterized by the constants in the matrix $Q$ and the scalar $R$, which thus can be seen as filter design variables. A larger value in one of $Q$ 's components means that the corresponding state variable(s) are more willing to change during the time between two time instants, whereas a larger $R$ corresponds to less measurement accuracy. Hence, larger $Q$ results in larger $K$ and larger $R$ results in a filter which puts less trust in the measurements and $K$ is therefore chosen smaller yielding a "slow" filter. The choice of $Q$ versus $R$ is thus a trade off between tracking and noise suppression. The design parameter $R$ should be chosen such that it approximately equals the variance of the residuals.

Another design choice concerns the update rate of the filter. A reason for not choosing a faster update rate, which of course provides a possibility for earlier detection of large changes in the relative load, is the increased computational burden a higher update rate induces. So perhaps updating every sample is not an obvious choice. We have studied filter that has an update rate of every tenth or every hundredth estimate sample. Simulations show that the performance in steady state when filtering is the same regardless of the update rate.

The behaviour of the CUSUM detector is primarily decided by the parameters $h$ and $\nu$. These have been chosen in such a way that we detect sudden changes of considerable amplitude within a reasonable time without having too many false alarms. In an attempt to 
make the detection time independent of the update rate of the filter, $h$ is chosen inversely proportional to the time between updates, i.e.,

$$
h=\frac{T_{\text {frame }}}{T} h_{0},
$$

where $h_{0}$ is the threshold used when updating every frame. In case of a change detection, the values of $P$ corresponding to $\tilde{x}_{1}$ and $\tilde{x}_{2}$ are increased a factor five, which results in making filtered value of these states more sensitive to the current input to the filter, $y(t)$. The motivation for increasing only these two states is that the change is believed to be in the load level and not in the AR parameters.

\subsubsection{Simulations}

Input data for this estimation application is taken from simulations produced by the simulator explained in Appendix A.1. A Kalman filter is used to extract the load level $\bar{L}$ from the signal $y$ in (9.5). By using the change detection technique described in Section 9.1 the filter is able to follow any sudden jumps in the load estimate.

Figure 9.5a shows the approximation of $L$ in (4.3) where $\Lambda$ is approximated using (5.16c). Also shown in Figure 9.5a is the Kalman filter estimate. The filter estimate is updated every frame, so $T=T_{\text {frame }}=0.01 \mathrm{~s}$. The filtered estimate is free of oscillations and follows an underlying trend in the original estimate. Note how the filtered estimate jumps when there is a distinct jump in the raw estimate. Figure $9.5 \mathrm{~b}$ shows a close up version of the top plot together with a rough low pass filtered version of the original estimate. Both filters suppress noise equally well, but the low pass filtered signal adapts to the new load level much slower. In case of a sudden jump upwards, the slow acclimatization of the low pass filtered signal can be risky. In case of a jump downwards, the resource utilization can be improved compared to using the low pass filtered version.

As a comparison, Figure 9.5c shows the performance of the filter when using three different update rates; once every frame as above, once every tenth frame and once every hundred frame. In steady state, the three versions of the filtering provide almost identical outputs. But, due to its superior update rate, the version which is updated every frame detects the jump earlier. However, all three update rates adapt to the new load level far faster than the simple low pass filtered signal, also shown Figure 9.5c. Thus, a faster update rate is perhaps not crucial, especially when comparing with the low pass filtered version. The estimation process also provides an estimate of the derivative of the trend in the relative load which enables a more aggressive resource management.

Due to the anti alias filter applied before the Kalman filter, the measurement noise $e(t)$ is no longer white. Therefore, the residuals from the estimation process are not white either. This indeed indicates the need for a more complex model, for example an ARMA model. However, as the simulations show, the proposed filter still manages to provide a good estimate of the underlying trend in the relative load estimate.

\subsection{Background Noise Power Estimation}

Section 7.5 establishes that using inaccurate information about the background noise power leads to unwanted transfer of resources between base stations. This section is de- 
a)

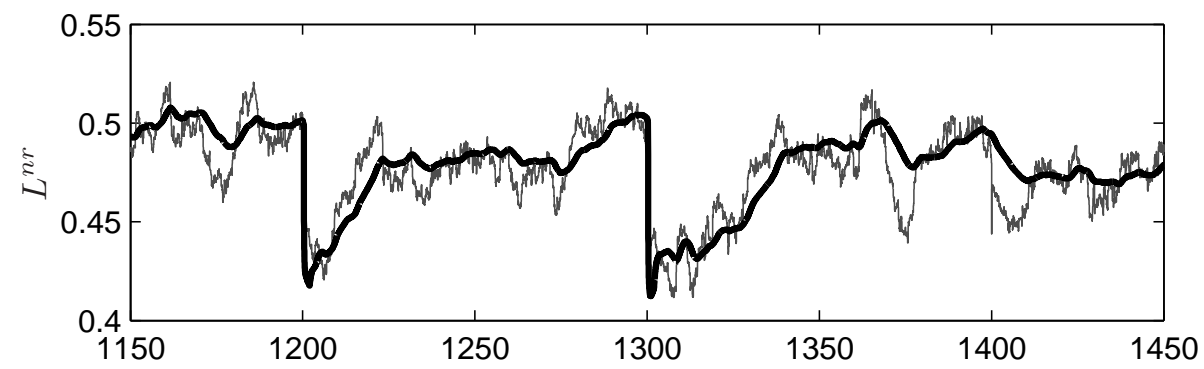

b)

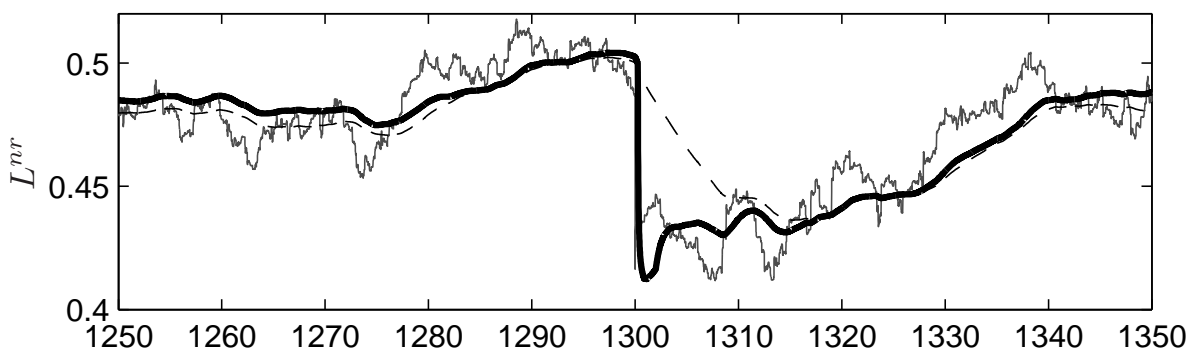

c)

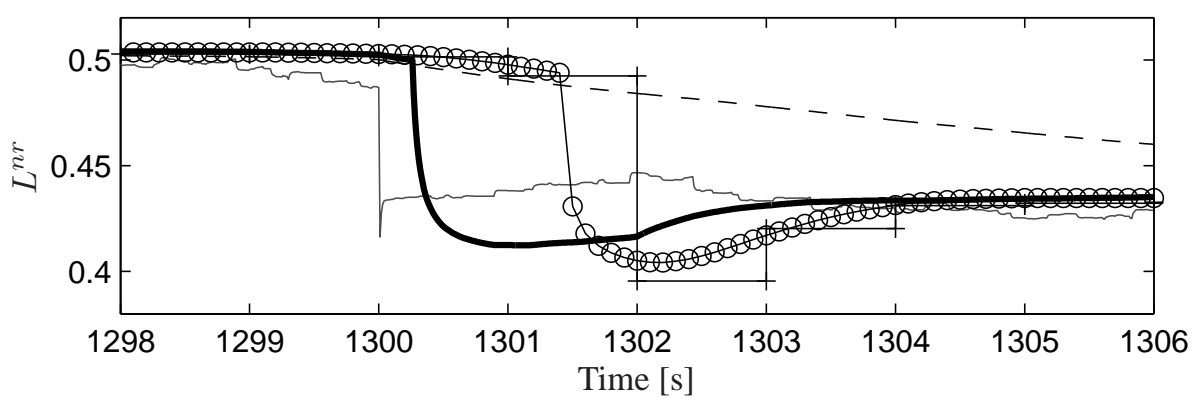

Figure 9.5: Performance of the Kalman filtering and change detection when applied to a signal with a rather big jump in it. The different plots are magnified versions of the same simulation. Solid: original estimate. Solid, thick: updated each frame. Solid, ring: updated every tenth frame. Solid, plus: updated every hundredth frame. Dashed: low pass filtered. 
voted to estimating the background noise power ratio, $\theta$, as defined in (7.13). An accurate estimate of the background noise power can, for example, be used by the load approximations based on iterations in Chapter 5. If the background noise powers are known, there is no need to assume equal background noise power during the derivation of the approximations.

Definition 6.6 introduces the system matrix. Properties of this matrix was repeatedly explored in Chapter 6. Here, the same framework is used to estimate the background noise power using measurements of the uplink interference power. Assuming that interference power measurements are available does in no way interfere with the motivation for studying uplink load approximations not using these measurements, as is done in Chapter 5. On the contrary, knowledge of the background noise power can be used instead of assuming a noise-flat system when deriving some of the approximations in Chapter 5. Furthermore, another reason for not using measurements of the uplink interference power for load approximations is that individual measurements may be associated with considerable errors, something which the background noise power application is not as sensitive to.

Unfortunately, measurements of the total uplink interference power is often corrupted by a bias in logarithmic scale, which also needs to be estimated. The state vector is thus

$$
x(t)=\left(\begin{array}{l}
\theta(t) \\
\delta(t)
\end{array}\right),
$$

where $\delta(t) \in \mathbb{R}^{B}$ is the vector with base station individual biases.

The biases and the background noise power are related to measurements of the total interference power in Section 9.3.1, where the relation is expressed as the measurement equation in a state space model. The state space model is derived under an assumption of connectivity one but this requirement is relaxed via an approximation in Section 9.3.2. A basic requirement for possibility of estimating the values in the state vector, is that the connection between them and measurements of the uplink interference power is strong enough, that they are observable. Even though the individual states are not observable, products of bias and background noise power ratio in the different base stations are observable, as is shown in Section 9.3.3. Fortunately, these are the products that will be used in practice anyway. Section 9.3.3 also contains a discussion on how the base stations can use these products together with measurements of the uplink interference power to estimate the current uplink noise rise. Finally, methods for nonlinear estimation are applied in Section 9.3.4.

The basis for this estimation is the system of nonlinear equations in (5.6). These equations lay the ground for the uplink load approximations in Chapter 5. Instead of solving for the uplink noise rise, as in Chapter 5, the equations are combined with measurements of the uplink interference power in order to estimate the background noise power.

\subsubsection{Connectivity One}

The nonlinearities in (5.6) are due to soft handover. In case of connectivity one, the system of nonlinear equations breaks down into a system of linear equations. It is shown in Section 6.1.2 that the system of equations then can be expressed in matrix form as

$$
I^{t o t}(t)=C(t) N(t), C(t) \triangleq\left(E-L(t)^{T}\right)^{-1}
$$


where $L(t)$ is the system matrix according to Definition 6.6. As opposed to the situation in Chapter 6, the time span studied here is too long for considering the users' target CTIR values and the relative power gain values as constant. In fact, it is crucial for this application that there are variations over time, as will be discussed in Section 9.3.3.

Element $j$ of the function $h(x, t)$ describing the relation between the states and the interference power measurement in base station $j$ is

$$
\begin{array}{r}
h_{j}(x(t), t) \triangleq 10 \log _{10}\left(N^{0} \delta_{j}(t) \sum_{k=1}^{B} C_{j, k}(t) \theta_{k}(t)\right) \\
=10\left(\log _{10}\left(N^{0}\right)+\log _{10}\left(\delta_{j}\right)+\log _{10} \sum_{k=1}^{B} C_{j, k}(t) \theta_{k}(t)\right) .
\end{array}
$$

As the base station error factor, $\delta_{j}, j=1,2 \ldots B$, and the background noise ratio, $\theta_{j}, j=1,2 \ldots B$ are modeled as integrated white noise, the system model becomes

$$
\begin{aligned}
\left(\begin{array}{c}
\theta(t+1) \\
\delta(t+1)
\end{array}\right) & =\left(\begin{array}{l}
\theta(t) \\
\delta(t)
\end{array}\right)+\left(\begin{array}{l}
w_{\theta}(t) \\
w_{\delta}(t)
\end{array}\right) \\
y(t) & =h(x(t), t)+e(t),
\end{aligned}
$$

with $x(t)$ given by (9.9).

The expression for the element on row $j$ and column $k$ of $H(t)$ used in the EKF depends on whether it relates to an element in $\theta$ or $\delta$.

$$
H_{j, k}(t)= \begin{cases}\left.\frac{\partial h_{j}(\delta, \theta)}{\partial \theta_{k}}\right|_{x=\hat{x}(t \mid t-1)}=\frac{10}{\log 10} \frac{C_{j, k}(t)}{\sum_{k=1}^{B} C_{j, k}(t) \hat{\theta}_{k}(t \mid t-1)} & \text { if } k \leq B \\ \left.\frac{\partial h_{j}(\delta, \theta)}{\partial \delta_{k-B}}\right|_{x=\hat{x}(t \mid t-1)}= \begin{cases}\frac{10}{\log 10} \frac{1}{\hat{\delta}_{j}(t \mid t-1)}, & k=j+B \\ 0, & k \neq j+B\end{cases} & \text { if } k>B .\end{cases}
$$

\subsubsection{Higher Connectivity}

In case of higher connectivity at least one mobile is power controlled by more than one base station. In this case it is not as easy to express the relation between measured interference power and the model states $N_{j}$ and $\delta_{j}$. This is due to the lack of an explicit solution to (5.6). As in Chapter 5, approximations can be applied to solve the system of nonlinear equations. Both the linearized and EKFs require an explicit expression for $h(x(t), t)$ in order to calculate $H(t)$. Therefore, fix point iterations to solve the system of nonlinear equations is not an option in this case.

A second way to solve the system of nonlinear equations is the one used in Section 5.2.2. This leads to an explicit, but approximative solution to the system of nonlinear equations. Basically, the denominator in the relative path gain, $z_{i, j}$, is substituted by a sum of path gain values taken over the set of cells that the user is connected to. This means that the element on row $k$ and column $j$ in the system matrix, $L(t)$, in (9.10) is substituted by

$$
\tilde{L}_{k, j}(t) \triangleq \sum_{i \in c_{k}} \beta_{i}^{t g t}(t) \frac{g_{i, j}(t)}{\sum_{\ell \in K_{i}} g_{i, \ell}(t)}
$$


Using the matrix $\tilde{L}=\left[\tilde{L}_{k, j}\right]$, the interference power can be approximated by an expression similar to $(9.11)$

$$
h_{j}(x(t), t) \triangleq 10 \log _{10}\left(N^{0} \delta_{j}(t) \sum_{k=1}^{B} \tilde{C}_{j, k}(t) \theta_{k}(t),\right.
$$

where $\tilde{C}(t) \triangleq(E-\tilde{L}(t))^{-1}$.

The particle filter can be applied with either of the methods discussed above, i.e., both fix point iterations or using the sum of path gain values in the denominator of relative power gain values. However, no adapts of estimating the background noise power when using fix point iterations have been made in the simulations.

\subsubsection{Estimation Limitations}

A necessary condition for estimation is observability. Loosely speaking, a system is observable if the connection between outputs and internal states is strong enough to uniquely determine the value of the internal states given the outputs. In our model, (9.12), the internal states are the $2 B$ variables found in the vectors $\delta$ and $N$.

Definition 9.1 (Observability). An internal state (function of states) is observable if, given measurements at time instants up until $t_{0}$, it is possible to establish what the state (function of states) is at time $t_{0}$.

Merely by looking at the model, one can conclude that it is impossible to determine the states uniquely since the biases, $\delta_{j}$, always appear multiplied by a background noise power ratio $\theta_{k}$, e.g., $\delta_{1} \theta_{1}, \delta_{1} \theta_{2}$ etc.

However, below it is shown that the products of biases and background noise power ratio are observable, i.e., that it is possible to estimate them based on measurements $y_{j}(t)$. Specifically, it is products of type $\delta_{j} \theta_{j}$ that are of interest. If these products are known, it is possible to calculate the noise rise based on measurements of the uplink interference power,

$$
\Lambda_{j}(t)=\frac{I_{j}(t)}{N(t)}=\frac{y_{j}(t) / \delta_{j}(t)}{\theta_{j}(t) N^{0}}=\frac{y_{j}(t)}{\delta_{j}(t) \theta_{j}(t) N^{0}} .
$$

The products are observable if, given a number of measurements $y(t), t=1,2, \ldots$, it is possible to establish the value of the products uniquely. It is much easier to show that the products are observable if the relation between them and a function of the measurements is linear. The function obviously has to be one-to-one. Be choosing the function to

$$
y^{l i n}=10^{y / 10},
$$

the relation between $y^{l i n}$ and the products is linear. Applying the conversion to the measurements of the model (9.12) in a noise less scenario yields, at time $t$,

$$
y_{j}^{l i n}(t)=\sum_{k=1}^{B} C_{j, k}(t) \delta_{j}(t) \theta_{k}(t) N^{0}, j=1,2 \ldots, B .
$$


Under the assumption that $\delta_{j}$ and $\theta_{j}$ are approximately constant over a period of time $t=t_{0}, t_{0}+1, \ldots, t_{1}$, consider the following system of equations.

$$
\begin{gathered}
y_{j}^{l i n}\left(t_{0}\right)=N^{0} \sum_{k=1}^{B} C_{j, k}\left(t_{0}\right) \delta_{j}\left(t_{0}\right) \theta_{k}\left(t_{0}\right), j=1,2 \ldots, B \\
y_{j}^{l i n}\left(t_{0}+1\right)=N^{0} \sum_{k=1}^{B} C_{j, k}\left(t_{0}+1\right) \delta_{j}\left(t_{0}\right) N_{k}\left(t_{0}\right), j=1,2 \ldots, B \\
\vdots \\
y_{j}^{l i n}\left(t_{1}\right)=N^{0} \sum_{k=1}^{B} C_{j, k}\left(t_{1}\right) \delta_{j}\left(t_{0}\right) N_{k}\left(t_{0}\right), j=1,2 \ldots, B .
\end{gathered}
$$

Skipping the time indices for $\delta_{j}$ and $\theta_{j}$, these equations can be put in matrix form as

$$
\frac{1}{N^{0}}\left(\begin{array}{c}
y_{1}^{l i n}\left(t_{0}\right) \\
y_{2}^{l i n}\left(t_{0}\right) \\
\vdots \\
y_{B-1}^{l i n}\left(t_{0}\right) \\
y_{B}^{l i n}\left(t_{0}\right) \\
y_{1}^{l i n}\left(t_{0}+1\right) \\
y_{2}^{l i n}\left(t_{0}+1\right) \\
\vdots \\
y_{B-1}^{l i n}\left(t_{1}\right) \\
y_{B}^{l i n}\left(t_{1}\right)
\end{array}\right)=\mathcal{O}\left(\begin{array}{c}
\delta_{1} N_{1} \\
\delta_{1} N_{2} \\
\vdots \\
\delta_{1} N_{B} \\
\delta_{2} N_{1} \\
\delta_{2} N_{2} \\
\vdots \\
\delta_{B} N_{B}
\end{array}\right)
$$

where

$$
\mathcal{O} \triangleq\left(\begin{array}{ccccc}
C_{1}\left(t_{0}\right) & 0 & \ldots & \ldots & 0 \\
0 & C_{2}\left(t_{0}\right) & 0 & \ldots & 0 \\
\vdots & \ddots & \ddots & \ddots & \vdots \\
\vdots & \ddots & \ddots & C_{B-1}\left(t_{0}\right) & 0 \\
0 & \ldots & \ldots & 0 & C_{B}\left(t_{0}\right) \\
C_{1}\left(t_{0}+1\right) & 0 & \ldots & \ldots & 0 \\
0 & \ddots & \ddots & \ldots & 0 \\
\vdots & \ddots & \ddots & \ddots & \vdots \\
\vdots & \ddots & \ddots & C_{B-1}\left(t_{1}\right) & 0 \\
0 & \ldots & \ldots & 0 & C_{B}\left(t_{1}\right)
\end{array}\right) .
$$

With $C_{j}(t)$ being the $j$ :th row of $C(t)$. Below are two different cases where the matrix $\mathcal{O}$ has full rank.

- As the cross products in the right hand side of (9.13) are not of interest, it is satisfactory to be able to solve for products of type $\delta_{j} \theta_{j}$. If, at time instant $t^{\prime}, C\left(t^{\prime}\right)$ 
is diagonal with all diagonal elements being nonzero, it is possible to estimate all $\delta_{j} \theta_{j}$ from that time instant alone.

$$
y_{j}^{l i n}\left(t^{\prime}\right)=C_{j, j}\left(t^{\prime}\right) \delta_{j} \theta_{j} N^{0}, j=1,2, \ldots, B .
$$

- If $C(t)$ is not diagonal, it is necessary to solve for products of type $\delta_{j} \theta_{k}$ as well. Equation (9.13) has a unique solution if $\mathcal{O}$ has full rank. As there are $B^{2}$ different products in the vector in the right hand side, we need measurements from at least $B$ different time instants. Furthermore, the $C$-matrix has to change over time in order to generate different measurement equations (different rows) at different times, $t=$ $t_{0}, t_{0}+1, \ldots, t_{2}$.

There are of course other cases where we can solve for the interesting products. The second case above is however the case interesting in practice, since we in practice can be expected to always have some active users in each cell. The main conclusion from the discussion in this section is summarized in the Theoreom 9.1

\section{Theorem 9.1 (Observability)}

If $\mathcal{O}$ achieves full rank within a time frame in which $\delta_{j}(t)$ and $N_{j}(t)$ may be assumed constant for all $j$, it is possible to estimate all products $\delta_{j} \theta_{j} \forall j=1,2, \ldots, B$.

Proof: If $\mathcal{O}$ has full rank, it is possible to solve for all products in the vector in the right hand side of (9.13). Specifically, it is possible to determine $\delta_{j} \theta_{j}, \forall j=1,2, \ldots, B$.

The requirement that $\mathcal{O}$ must achieve full rank is met if $C(t)$ varies in a random fashion over time. This is the case if traffic is rapidly changing, e.g., due to fast scheduling.

\subsubsection{Simulations}

Once again, the simulator discussed in Appendix A.1 is used for producing simulation data. This time realistic interference power measurements are gathered together with information about the users' target CTIR values and their path gain values to base stations. All values are averaged over a time frame of 100 milliseconds in the base stations before being delivered to the central node where the actual estimation process takes place.

As the measurements are averaged over a relatively long time period, compared to both the fast variations in radio channel between users and base stations and the rapid variations in users' transmission rates, the measurements do not capture momentarily interference power peaks. Simulations indicate that it is still possible to accurately estimate the products of the base station's individual biases, $\delta_{j}$, and their background noise power ratios, $\theta_{j}$.

Table 9.1 and Table 9.2 show the statistics of 15 Monte Carlo simulations with 20000 samples in each simulation. The root mean square error (RMSE) as defined by Gustafsson (2000) has been calculated as

$$
R M S E=\left(\frac{1}{M C} \sum_{\ell=1}^{M C} \frac{1}{B} \sum_{j=1}^{B} \frac{1}{T} \sum_{t=1}^{T}\left\|\hat{\theta}_{j}^{(\ell)}(t)-\theta_{j}^{\ell}(t)\right\|_{2}^{2}\right)^{1 / 2},
$$


where $\theta_{j}^{\ell}(t)$ is the true background noise power ratio in base station $j$ at time $t$ in Monte Carlo simulation $\ell$ and $[1, T]$ is the interval over which statistics is studied. When calculating the statistics in the table, the first 20 seconds of each simulation has been removed.

The two particle filters, PF1 and PF2, have 5000 and 10000 particles, respectively. The two filters provide roughly the same statistics, meaning that there is either not much to gain from adding even more particles, or the number of particles is far too small. The extended Kalman filter actually provides slightly better performance in the case of connectivity one, which is the case that the model is designed for. The particles filters, however, seems slightly more robust against the model error that connectivity three brings. Out of the proposed filters, all save for the linearized Kalman filter provides good estimation error statistics. Using an ordinary Kalman filter applied to a linearized model does not seem to work at all in this application. The true product of $\delta_{j}$ and $\theta_{j}$ is probably too far from the chosen working point. The relatively poor performance of the particle filter, compared to the extended Kalman filter, may be explained by a property of particle filters yielding that they perform worse when estimating parameters of a model with small process noise and many states (Gustafsson et al., 2002). The model contains 18 states and the process noise is practically zero since both $\theta$ and $\delta$ are assumed to be approximately constant. Gustafsson et al. (2002) claims that it can be worth trying even more particles in this case.

As the users' path gain values are both averaged over quite a few time instants and do not include the multi path fading, the model does not consider the TX increase. Furthermore, the TX increase is larger when using connectivity one compared to using connectivity three. This may explain some of estimation improvement when going from connectivity one to connectivity three. Another reason may be the decreased intensity of high interference power peaks when using connectivity three.

Table 9.1: Estimation errors when estimating the product of background noise ratio and the base station individual biases. PF1 and PF2 are particle filters with 5000 and 10000 particles, respectively. Connectivity one. 15 Monte Carlo simulations.

\begin{tabular}{|c|c|c|c|}
\hline & Mean & Var. & RMSE \\
\hline EKF & 0.05 & 0.04 & 0.21 \\
\hline PF1 & 0.07 & 0.08 & 0.29 \\
\hline PF2 & 0.07 & 0.09 & 0.30 \\
\hline
\end{tabular}

Table 9.2: Estimation errors when estimating the product of background noise ratio and the base station individual biases. PF1 and PF2 are particle filters with 5000 and 10000 particles, respectively. Connectivity three. 15 Monte Carlo simulations.

\begin{tabular}{|c|c|c|c|}
\hline & Mean & Var. & RMSE \\
\hline EKF & -0.09 & 0.02 & 0.18 \\
\hline PF1 & -0.06 & 0.02 & 0.14 \\
\hline PF2 & -0.06 & 0.01 & 0.13 \\
\hline
\end{tabular}

The statistics in Table 9.1 and Table 9.2 are calculated in linear domain. Correspond- 
ing results for the case of connectivity three in logarithmic scale are given in Table 9.3. The table shows that the particle filters provide a smaller RMSE corresponding to a more reliable estimate. Furthermore, discussing the absolute numbers, an average error of $0.4 \mathrm{~dB}$ and an error variance of 0.6 means that the estimate is less than one $\mathrm{dB}$ wrong in approximately two thirds of the considered time instants. This is rather small considering that the background noise takes values in a range of about three $\mathrm{dB}$ and that there is a measurement bias on top of that. Figure 9.6 shows how the different estimates evolve

Table 9.3: Estimation statistics in logarithmic scale $(d B)$ for connectivity three.

\begin{tabular}{|c|c|c|c|}
\hline & Mean & Var. & RMSE \\
\hline EKF & -0.80 & 4.26 & 2.21 \\
\hline PF1 & -0.38 & 0.58 & 0.85 \\
\hline PF2 & -0.45 & 1.01 & 1.10 \\
\hline
\end{tabular}

over time. At $t=20 \mathrm{~s}$, the true background noise power jumps up with three $\mathrm{dB}$ and at $t=40 \mathrm{~s}$ it jumps back down again. The linearized Kalman filter is not shown in the picture. The jump up corresponds to the true background noise ratio making a jump from one to two. This jump corresponds to halve $\rho$ defined in Section 7.5. It is certainly not possible to relate the jump in Figure 9.6 to a certain decrease in the estimated background noise ratio in for example Figure 7.6. Even so, it is still clear that if such a jump is not adapted to by a base station using a noise rise controlling algorithm, the base station's capacity will decrease considerably. Figure 9.6 shows that the extended Kalman filter and the two particle filters manage to adapt to the new background noise power level, despite the sudden and substantial jump. Thus, if using the proposed technique for estimating the background noise power, it possible for a noise rise controlling algorithms to eventually operate normally despite the sudden jump in the background noise level.

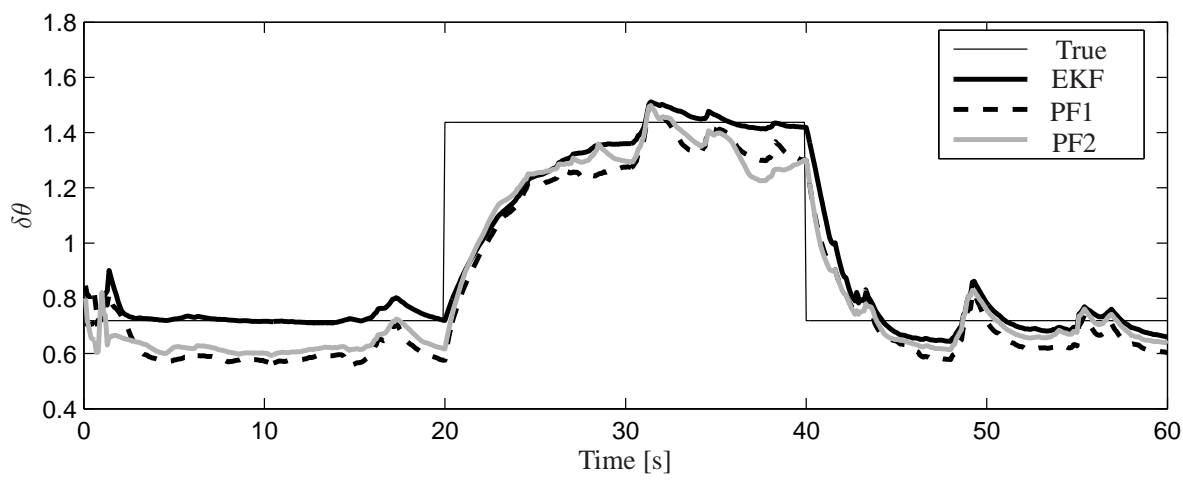

Figure 9.6: Estimation of $\theta \delta$. The true background noise power makes jumps at $t=20 \mathrm{~s}$ and $t=40 \mathrm{~s}$. The particle filters have 5000 (PF1) and 10000 (PF2) particles. The filters are initialized with a product equal to one. A system with connectivity three has been simulated. 


\subsubsection{Implementation Aspects}

When calculating $C(t)$, the system matrix, $L(t)$, as in Definition 6.6 is used. The system matrix consists of ratios between users' power gains. In practice, the central node is unlikely to receive information on the users' instantaneous multi path fading. One way of still considering this fast changing part of the power gain is to compile the system matrix in the base stations.

Loosely speaking, the element on row $k$ and column $j$ of the system matrix represents the load cell $k$ causes in cell $j$. Since the base station receives frequent reports on the power gains between users and several base stations, each base station $k$ can, on its own, calculate values representing an average of these load contributions,

$$
\tilde{L}_{k, j}=\frac{1}{T} \sum_{\tau=1}^{T} \sum_{i \in c_{k}} \frac{g_{i, j}(\tau)}{g_{i, k}(\tau)} \beta_{i}^{t g t}(\tau) .
$$

Here, $T$ is the number of time instants between each report from the base stations to the central node. Based on these reports, the central node would then compile the system matrix and then use it to calculate $C(t)$.

\subsection{Summary}

Due to user movement, the noise rise relative load is subject to slow oscillations. Earlier work has provided an approximation of the noise rise relative load which to a great extent captures these oscillations. In this chapter, a signal model which describes the noise rise relative load as a signal consisting of a bias with a trend together with a fourth order auto regressive model has been developed. A Kalman filter together with a change detection algorithm has been applied to the model. The result is a stable signal representing a time average of the uplink load. Unlike what an ordinary low pass filter would provide, the filter is still alert on sudden changes in the load level due to the change detection algorithm used. Furthermore, the filter provides an indication towards where the load is heading. This information enables more aggressive resource management, which in the end enables better resource utilization.

A straight forward way of controlling the uplink noise rise level is to measure the uplink interference power and divide it by a number representing the background noise power. Two problems with this are that the interference power measurements are often corrupted by a bias in logarithmic scale and that the background noise power is unknown. The second application of this chapter is a way of centrally estimate the product of measurement bias and background noise power in the different base stations. This is done by using advanced signal processing together with measurements of the interference power and path gain between users and base stations. Simulations indicate that it is possible to satisfactory estimate the product, despite the fact that both multi path fading and soft handover is considered in the simulations but not in the model used in the estimation process. Using this method, the base stations can calculate the uplink noise rise by measuring the uplink interference power and divide it by the estimated product. This gives, not only sensitivity to variations in the background noise power, but also robustness to the interference power measurement bias. Better knowledge of the background noise power enables 
better resource utilization as argued in Chapter 8. Furthermore, if the measurement bias can be eliminated, the need for it to be small is not as crucial for good performance. 


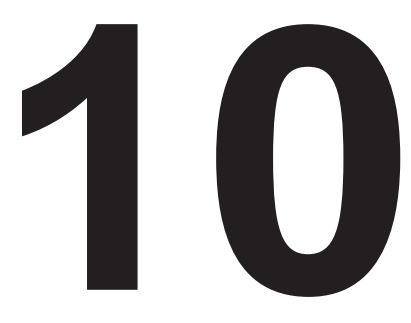

\section{Conclusions}

Due to the nature of code division multiple access (CDMA), the uplink load of CDMA cellular radio system should be related to either received or transmitted interference power. In this thesis, load definitions based on both of these approaches are proposed and relations between them explored. Intercell interference, i.e., interference received in or caused by users connected to another cell, is studied in all parts of the thesis.

A number of load approximations that explicitly consider the received intercell interference contribution to the uplink load are proposed. The approximations are independent of interference power measurements through the use of a model for the received interference power together with users' path gain reports. A time averaged load can be approximated with negligible errors. However, the approximations use sparsely sampled information and can therefore not relate to momentarily load levels. A resource control algorithm based on the approximations can thus utilize the system's soft capacity, but can not adapt to fast variations in the radio environment.

A system matrix, describing the relation between users' radio environment, background noise power and the received interference power is developed and properties of it are repeatedly used. A first application is to establish a number of relations between the different uplink load definitions. These include a load definition related to system feasibility, i.e., whether there exists transmission power levels to support the resource demands. Relations between the load definitions and the load approximations are also derived. This analysis yields that iterations involved in the load approximations converge to the true uplink load under certain requirements on the true system.

It is generally acknowledged that the high control bandwidth required to adapt to fast variations in users' radio environment can be achieved through decentralized control. Properties of the system matrix are used to design a number of decentralized resource allocation algorithms. As these algorithms control the amount of transmitted intercell interference, they are robust in the sense that system feasibility is guaranteed. Simulations indicate that the proposed algorithms provide equal performance as a completely 
centralized algorithm with complete knowledge of all users' radio environment.

A straightforward way of controlling the uplink load is to measure the amount of received interference power and divide by the background noise power. Yet another application of the system matrix is to estimate the background noise power based on measured interference power. The resulting estimation errors are negligible compared to the interference power measurement errors. 


\section{A}

Appendix

\section{A.1 Cellular Radio System Simulator}

Properties of the simulator will be described in this section. First, algorithms and parameters of the radio environment as well as user creation, movement and transmission patterns will be described. Later, properties of the system such as base station and cell configuration are given together with resource management algorithms and parameters.

A wide range of algorithms and characteristics of a CDMA system is simulated, from algorithms operating at a short time scale such as power control and multi path fading to mechanisms/algorithms naturally operating on a far longer time scale such as user creation and link configuration. The simulator is an improved version of the simulator included in Zander et al. (2001).

\section{A.1.1 Models}

Attenuation. The simulator models the total power gain as a product of four parts; antenna gain, distance attenuation, shadow fading and multi path fading. The three first parts are considered fixed for a certain position in the simulator area throughout the entire simulations. In the factor representing the antenna gain, a direction-dependent attenuation is modeled. The distance attenuation, or path loss, is parameterized through the path loss coefficient, $\alpha=3.5$ and the shadow fading through $d_{0}=100 \mathrm{~m}$ and $\sigma=6 \mathrm{~dB}$ in the models given in Section 3.1. Multi path fading is updated with a function of traveled distance every simulation step. This is used to model the stochastics in the multi path fading even for a given point in space. On top of this an extra component is used to model the performance of the link estimation algorithms of a real system. This factor is a function of the user's speed. Characteristics of the multi path fading model is given in (3GPP, 2000c) under the name of "3GPP Typical Urban". A rake receiver with three fingers is incorporated in the model. 
User Movement and Transmission Behavior. The mobility model states that the users move with a constant absolute velocity but with angular variations. There are two types of transmission behaviors. Both of them use full buffers, i.e., users always have data to send.

In the first one, admitted users are continuously transmitting between the time of admission until their they have send all their data. The amount of data to send is randomly chosen from an exponential distribution. This is an example of slow radio resource management (RRM).

Fast RRM is modeled in the second model, meaning that the users send only when explicitly told to do so. No specific amount of data is modeled in this case.

\section{A.1.2 Other Features}

Power Control The simulator updates the transmission power of all users at every slot, i.e., at a rate of $1500 \mathrm{~Hz}$. Feedback delay is implemented and power control errors are modeled. The update rate is in agreement with what is done in WCDMA (3GPP, 2000b).

Radio Resource Management Session admission is performed every frame. Two different frame lengths have been used in the simulations. Slow RRM corresponds to a frame length of $10 \mathrm{~ms}$, and fast RRM corresponds to $2 \mathrm{~ms}$. The shorter frame length is used in an enhanced version of the WCDMA uplink (Parkvall et al., 2005).

\section{A.1.3 Simulator Utilization}

Various parts of the simulator are used for different purposes. Table A.1 summarizes what parts of the simulator that is used for different simulation purposes.

Table A.1: Summary of where the different parts of the simulator are used.

\begin{tabular}{|l|c|c|c|c|c|}
\hline & $\begin{array}{l}\text { Power } \\
\text { Control }\end{array}$ & $\begin{array}{l}\text { Power } \\
\text { Gain } \\
\text { Modeling }\end{array}$ & Mobility & $\begin{array}{l}\text { Slow } \\
\text { RRM }\end{array}$ & $\begin{array}{l}\text { Fast } \\
\text { RRM }\end{array}$ \\
\hline Section 5.7 & $\mathrm{x}$ & $\mathrm{x}$ & $\mathrm{x}$ & $\mathrm{x}$ & \\
\hline Section 7.1 & $\mathrm{x}$ & $\mathrm{x}$ & $\mathrm{x}$ & $\mathrm{x}$ & \\
\hline Section 7.4 & & $\mathrm{x}$ & & & \\
\hline Section 8.2.3 & & $\mathrm{x}$ & & & \\
\hline Section 9.2.4 & $\mathrm{x}$ & $\mathrm{x}$ & $\mathrm{x}$ & $\mathrm{x}$ & \\
\hline Section 9.3.4 & $\mathrm{x}$ & $\mathrm{x}$ & $\mathrm{x}$ & & $\mathrm{x}$ \\
\hline
\end{tabular}

\section{A.2 Positive Matrices Theory}

The characterizing property of positive matrices is that they have only positive elements. The following theorem can be found in (Gantmacher, 1974, page 66). All inequalities in this appendix should be interpreted component wise. 


\section{Theorem A.1}

A non-negative matrix A always has a non-negative eigenvalue $r$ such that the moduli of all the eigenvalues of $A$ do no exceed $r$. To this 'maximal' eigenvalue $r$ there corresponds a non-negative eigenvector

$$
A y=r y,(y \geq 0, y \neq 0) .
$$

The adjoint matrix $B(\lambda)=(\lambda E-A)^{-1} \Delta(\lambda)$ satisfies the inequalities

$$
B(\lambda) \geq 0, \frac{d}{d \lambda} B(\lambda) \geq 0 \text { for } \lambda \geq r .
$$

Another word for moduli is absolute value and $\Delta(\lambda)$ is the characteristic polynomial of $A, \Delta(\lambda)=|\lambda E-A|$.

Proof: See (Gantmacher, 1974).

Interesting consequences of the theorem related to this work are

- The maximum eigenvalue, i.e., the eigenvalue with largest absolute value, is real and positive

$$
\bar{\lambda}(A)=\max \operatorname{eig}(A)=\max |\operatorname{eig}(A)| \geq 0
$$

- The smallest real eigenvalue such that the inequality

$$
A y \leq r y
$$

has positive real solutions $y$ is the maximum eigenvalue.

- Choosing $\lambda=1$ in the theorem yields that

$$
(E-A)^{-1}=\frac{B(1)}{\Delta(1)}>0
$$

if the largest eigenvalue, $r$, is less than one and $A$ is an irreducible matrix.

A strictly positive matrix is always irreducible.

\section{A.3 Schur Complement}

The Schur Complement of a matrix is a useful tool for establishing whether a matrix is positive definite or not Zhou et al. (1995). Consider the matrix

$$
X=\left(\begin{array}{cc}
A & B \\
B^{T} & C
\end{array}\right),
$$

where $A$ is a symmetric matrix. Assume that $\operatorname{det}(A) \neq 0$ and define the Schur complement of $X$ as the matrix

$$
S=C-B^{T} A^{-1} B .
$$

By the construction of $S$, it follows that ${ }^{1} X \succeq 0 \Rightarrow S \succeq 0$ if $A \succ 0$. 


\section{A.4 Proof of Theorem 6.5}

Theorem 6.5 is repeated here for convenience.

Theorem 6.5 (Bound on Uplink Feasibility Relative Load) Consider a noise-flat system with connectivity one. The feasibility relative load, $L_{f}$ is bounded below and above by

$$
\max _{j} \sum_{\ell \in c_{j}} \beta_{\ell}^{t g t} \leq L_{f} \leq \max _{j} L_{j}^{I M R C} .
$$

The two different inequalities will be proven in separate lemmas.

\section{Lemma A.1}

In a system with connectivity one,

$$
L_{f} \leq \max _{j} L_{j}^{I M R C},
$$

with equality in a single cell scenario.

Proof: In a system with connectivity one, $L_{j}^{I M R C}$ can be expressed in vector form as

$$
L_{j}^{I M R C}=\sum_{i=1}^{M} \beta_{i}^{t g t} z_{i, j}=\sum_{k_{1}}^{B} \sum_{i \in c_{k}} \beta_{i}{ }^{t} g t z_{i, j}=\sum_{k=1}^{B} L_{k, j} .
$$

The greatest approximation can then be expressed as

$$
\max _{j} L_{j}^{I M R C}=\|L\|_{1} .
$$

Theorem A.4 in Skogestad and Postlethwaite (1996) states that for any matrix norm

$$
\max |\operatorname{eig}(L)| \leq\|L\| \text {. }
$$

According to Theorem 6.2, $L_{f}=\max _{j}|\operatorname{eig}(L)|$ in case of connectivity one.

In case of a single cell scenario, the matrix $L=\sum_{i} \beta_{i}^{t g t}$, hence so thus $L_{f}$ which proves that equality holds in a single cell scenario.

\section{Lemma A.2}

In a system with connectivity one,

$$
\max _{j} \sum_{\ell \in c_{j}} \beta_{\ell}^{t g t} \leq L_{f}
$$

with equality in and only in a single cell scenario.

Proof: The cases of single cell and multi cell are studied separately. The equality requirement in case of a single cell system has already been handled the proof of Lemma A.1. Strict inequality is required in the multi cell case. The left hand side of the expression in the lemma can be reformulated as $\max _{j} L_{j, j}$. As $L_{j, j}$ is a principal minor of $L$ in case of multi cell system, $L_{j, j}$ is strictly less than the maximum eigenvalue of $L$ (Gantmacher, 1974). This proves the strict inequality in the multi cell case and claim in the lemma is proved.

\footnotetext{
${ }^{1}$ The notation $A \prec B$, where $A$ and $B$ are matrices, means that the maximum eigenvalue of $A-B$ is less than 0 .
} 
Proof: (Theorem 6.5)

The theorem follows from Lemma A.1 and Lemma A.2.

\section{A.5 Proof of Theorem 6.7}

Consider the update function in Algorithm 6.1 in the case of connectivity one,

$$
\begin{gathered}
\Lambda_{j}(t, n)=1+\sum_{i=1}^{M} \beta_{i}^{t g t}(t)+\frac{g_{i, j}(t)}{\sum_{k \in K_{i}} \frac{g_{i, k}(t)}{\Lambda_{k}(t, n-1)}}= \\
1+\sum_{i=1}^{M} \beta_{i}^{t g t}(t) \frac{g_{i, j}(t)}{g_{i, K_{i}}} \Lambda_{K_{i}}=1+\sum_{i=1}^{M} \beta_{i}^{t g t}(t) z_{i, j} \Lambda_{K_{i}} .
\end{gathered}
$$

As fixed point iterations are considered, the time index is dropped, $t$, for the target CTIR values, $\beta_{i}^{t g t}$. Furthermore, the above expression for base station $j$ can be written for all base stations in matrix form as

$$
\Lambda(t, n)=1+Z^{T} B K \Lambda(t, n-1)=1+L^{T} \Lambda(t, n-1) .
$$

This linear recursion will converge, regardless of initialization point, if the eigenvalues of $L$ are all inside the unit circle. Finally, the system as the system is assumed feasible,

$$
L_{f}<1 \Rightarrow \max \operatorname{eig}(L)<1 \text {. }
$$

The convergence point is found by inserting a constant $\Lambda$, independent of the index $n$, into the above update formula.

$$
\Lambda=1+L^{T} \Lambda
$$

This equation is the same as (6.3) in case of perfect power control and a noise-flat system. The convergence point of Algorithm 6.1 thus equals the true noise rise in a noise-flat system with perfect power control.

\section{A.6 Proof of Theorem 6.8}

\section{A.6.1 Preliminaries}

Introduce $f(x)$ as the update function of Algorithm 6.1,

$$
f_{j}(x) \triangleq 1+\sum_{\ell=1}^{M} \beta_{\ell}^{t g t} \frac{g_{\ell, j}}{\sum_{k=1}^{B} \frac{K_{\ell, k} g_{\ell, k}}{x_{k}}}, j=1,2 \ldots, B .
$$

\section{Lemma A.3}

When Algorithm 6.1 is initialized with a $\Lambda(t, 0) \leq \Lambda$ and applied to a noise-flat feasible system, the iterations are bounded above by the finite true noise rise vector, $\Lambda$. 
Proof: (Lemma A.3)

The theorem states that

$$
f(\Lambda(t, 0)) \leq \Lambda \text { if } \Lambda(t, 0) \leq \Lambda .
$$

Note that each $f_{j}(x), j=1,2 \ldots, B$ is a non-decreasing function in $x$. It is thus sufficient to show that $f(\Lambda) \leq \Lambda$. Introduce

$$
\hat{p}_{\ell}(x) \triangleq \frac{\beta_{\ell}^{t g t}}{\sum_{k=1}^{B} \frac{K_{\ell, k} g_{\ell, k}}{x_{k}}} .
$$

Next it is shown that $\hat{p}_{\ell}(x)$ is less than or equal to the true transmission power, $p_{\ell}$.

The actual power control algorithms are based on measurements of the the user's carrier-to-interference ratio (CIR), $\gamma_{\ell}$, instead of its CTIR. In case of maximum ratio combining, the sum of the locally received CIR-values is studied. Because of the nonlinear relation between CTIR and CIR, $\beta_{\ell}^{t g t}=\gamma_{\ell}^{t g t} /\left(1+\gamma_{\ell}^{t g t}\right)$, a combined CIR equal to the target CIR does not imply that the combined CTIR equals the target CTIR, despite perfect power control. The implication is true only in systems with connectivity one (i.e., when the total received CTIR consist of just one received signal). In general the combined CTIR is greater than or equal to the target CTIR,

$$
\begin{gathered}
\sum_{k \in K_{\ell}} \beta_{\ell, k}=\sum_{k \in K_{\ell}} \frac{p_{\ell} g_{\ell, k}}{I_{k}}=\sum_{k \in K_{\ell}} \frac{\gamma_{\ell, k}}{1+\gamma_{\ell, k}} \\
\geq \sum_{k \in K_{\ell}} \frac{\gamma_{\ell, k}}{1+\gamma_{\ell}}=\frac{\gamma_{\ell}}{1+\gamma_{\ell}}=\beta_{\ell}=\beta_{\ell}^{t g t},
\end{gathered}
$$

where $\beta_{l, k}$ and $\gamma_{l, k}$ are the locally received CTIR and CIR, respectively. Solving for $p_{\ell}$ above yields

$$
p_{\ell} \geq \frac{\beta_{\ell}^{t g t}}{\sum_{k=1}^{B} \frac{K_{\ell, k} g_{\ell, k}}{I_{k}}}=\hat{p}_{\ell}(I)=\hat{p}_{\ell}(\Lambda) N .
$$

Dividing the inequality by the common background noise $N$ and inserting it into (A.3) yield

$$
f_{j}(\Lambda)=1+\sum_{\ell=1}^{M} \hat{p}_{\ell}(\Lambda) g_{\ell, j} \leq 1+\sum_{\ell=1}^{M} \frac{p_{\ell}}{N} g_{\ell, j}=\Lambda_{j} .
$$

Finally, all elements of $\Lambda$ are obviously finite since the system is feasible.

\section{Lemma A.4}

The update function of Algorithm 6.1, $f(x)$, is a standard interference function as defined in Yates (1995).

See Section A.6.3 for an introduction to standard interference functions.

Proof: The statement follows by simply checking that $f(x)$ complies with the requirements of a standard interference function,

- Positivity This property is obvious considering that $f(x)$ represents a noise rise level. 
- Monotonicity Assume $x^{\prime} \geq x$ and study the denominator of an arbitrary user $\ell^{\prime}$ s contribution to the sum in (A.3),

$$
\sum_{k=1}^{B} \frac{K_{\ell, k} g_{\ell, k}}{x_{k}^{\prime}} \leq \sum_{k=1}^{B} \frac{K_{\ell, k} g_{\ell, k}}{x_{k}} .
$$

Since the denominator of term in (A.3) is either unchanged or decreased, user each user $\ell$ 's contribution is either unchanged or increased. Thus,

$$
\begin{array}{r}
f_{j}\left(x^{\prime}\right)=1+\sum_{\ell=1}^{M} \beta_{\ell}^{t g t} \frac{g_{\ell, j}}{\sum_{k=1}^{B} \frac{K_{\ell, k} g_{\ell, k}}{x_{k}^{\prime}}} \\
\geq 1+\sum_{\ell=1}^{M} \beta_{\ell}^{t g t} \frac{g_{\ell, j}}{\sum_{k=1}^{B} \frac{K_{\ell, k} g_{\ell, k}}{x_{k}}}=f_{j}(x), j=1,2 \ldots B .
\end{array}
$$

- Scalability It suffices to consider one arbitrary component, $f_{j}(x)$, at a time. For component $f_{j}(x)$,

$$
\begin{aligned}
f_{j}(\mu x)=1 & +\sum_{i} \beta_{i}^{t g t} \frac{g_{i, j}}{\sum_{k=1}^{B} \frac{K_{\ell, k} g_{i, k}}{\mu x_{k}}}=1+\mu \sum_{i} \beta_{i}^{t g t} \frac{g_{i, j}}{\sum_{k=1}^{B} \frac{K_{\ell, k} g_{i, k}}{x_{k}}} \\
& <\mu\left(1+\sum_{i} \beta_{i}^{t g t} \frac{g_{i, j}}{\sum_{k=1}^{B} \frac{K_{\ell, k} g_{i, k}}{x_{k}}}\right)=\mu f_{j}(x) .
\end{aligned}
$$

The inequality is true since $\mu$ is greater than one.

\section{A.6.2 Proof of Theorem 6.8}

Proof: (Theorem 6.8)

The theorem claims that there is a solution, $x$, to the equation $x=f(x)$ if the system is feasible. According to Lemma A.3 the iterations in Algorithm 6.1 are bounded by the compact, convex set $C=\left\{x \in \mathbb{R}^{B}: 1 \leq x_{j} \leq \Lambda_{j}\right\}$, if initialized with $x_{j}(0)=1 \forall j$ and applied to a noise-flat feasible system. Furthermore, $f(x)$ is a continuous function on $C$. Therefore, according to Brouwer's fixed point theorem (Ortega and Rheinboldt, 1970), there exists a vector, $x_{0}$, such that $x_{0}=f\left(x_{0}\right)$.

According to Lemma A.4, the existence of a solution to the equations and Theorem 2 in Yates (1995) which is stated in Section A.6.3 below, the iterations in Algorithm 6.1 will converge to $x_{0}$.

\section{A.6.3 Standard Interference Functions}

Yates (1995) shows that, given the three conditions in the theorem below, the equilibrium point $x_{*}$ is unique in the entire $\mathbb{R}^{n}$. Furthermore, the convergence is independent of the initialization point. 


\section{Theorem A.2 (Global Convergence)}

Assume that there exist a $x_{*}$ such that $f\left(x_{*}\right)=x_{*}$ and $f(x)$ satisfies the following properties

- Positivity: $f(x)>0$

- Monotonicity: If $x \geq x^{\prime}$ then $f(x) \geq f\left(x^{\prime}\right)$

- Scalability: For all $\mu>1, \mu f(x)>f(\mu x)$.

Then the iteration $x(t+1)=f(x(t))$ converges to $x_{*}$ for all $x(0) \in \mathbb{R}^{n}$.

The above inequalities should be interpreted component wise.

Proof: See Yates (1995).

\section{A.7 Proof of Lemma 7.2}

The schur complement discussed in Appendix A.3 will be used to prove the lemma. Comparing $X$ in (A.1) with the extended matrix in Lemma (7.5) yields

$$
A=E, C=L^{t g t^{2}} E \text { and } B=\bar{L} \text {. }
$$

Now, since $A=E \succ 0$, the requirement in the lemma implies

$$
L^{t g t^{2}} E-\bar{L}^{T} \bar{L} \succeq 0 \Leftrightarrow \bar{L}^{T} \bar{L} \preceq L^{t g t^{2}} E .
$$

This means that

$$
\lambda_{k}\left(\bar{L}^{T} \bar{L}\right) \leq L^{t g t^{2}}, k=1,2, \ldots B .
$$

As the maximum eigenvalue of a matrix is less than or equal to its maximum singular value (Golub and van Loan, 1996, page 318) this gives

$$
\bar{\lambda}(\bar{L}) \triangleq \max _{k} \lambda_{k}(\bar{L}) \leq \sqrt{\bar{\lambda}\left(\bar{L}^{T} \bar{L}\right)} \leq L^{t g t} .
$$




\section{Bibliography}

3GPP. Radio Resource Management. Technical Specification 3GPP TS 25.133, 3GPP, 2000a.

3GPP. Physical radio procedures. Technical Specification 3GPP TS 25.214, 3GPP, 2000b.

3GPP. FDD Enhanced Uplink; Overall description. Technical Specification 3GPP TS 25.309, 3GPP, 2005.

3GPP. RRC Protocol Specification. Technical Specification 3GPP TS 25.331, 3GPP, 1999.

3GPP. Physical radio procedures. Technical Specification 3GPP TR 25.943, 3GPP, 2000c.

Mehdi Alasti and Nariman Farvardin. A diffusion approximation for voice-data integration in a wireless network. In Proceedings of the IEEE Wireless Communications and Networking Conference, Chicago, IL, USA, Sep 2000.

Sirikiat Ariyavisitakul and Li Fung Chang. Signal and interference statistics of a CDMA system with feedback power control. IEEE Transactions on Communications, 41(11), 1993.

Fredrik Berggren and Riku Jäntti. Asymptotically fair scheduling on fading channels. In Proceedings of the IEEE Vehicular Technology Conference, 2002.

Patrick Billingsley. Probability and Measure. John Wiley \& Sons, LTD, 1995.

Peter J. Black and Qiang Wu. Link budget of cdma2000 1xEV-DO wireless internet access system. In Proceedings of the IEEE Personal, Indoor and Mobile Radio Communications Conference, September 2002. 
Hatem Boujemaa, Mohammed Siala, and Raphael Visoz. Multipath diversity and power control. In Proceedings of the IEEE Vehicular Technology Conference, Birmingham, Alabama, USA, May 2002.

Stephen Boyd and Lieven Vandenberghe. Convex Optimization. Cambridge University Press, The Edinburgh Building, Cambridge, CB2 2RU, UK, 2004.

Pete Boyer, Milica Stojanovic, and John Proakis. A simple generalization of the CDMA reverse link pole capacity formula. IEEE Transactions on Communications, pages 1719-1722, October 2001.

Jin-Ghoo Choi and Saewoong Bahk. Multiclass call admission control in QoS-sensitive CDMA networks. In Proceedings of the IEEE International Conference on Communications, Helsinki, Finland, June 2001.

Jelena Damnjanovic, Avinash Jain, Tao Chen, and Sandip Sarkar. Scheduling the cdma2000 reverse link. In Proceedings of the IEEE Vehicular Technology Conference, September 2002.

John Dennis Jr. and Robert B. Schnabel. Numerical Methods for Unconstrained Optimization and Nonlinear Equations. Prentice Hall, Englewood Cliffs, N.J., USA, 1983.

Nikos Dimitriou, Georgios Sfikas, and Rahim Tafazolli. Call admission policies for UMTS. In Proceedings of the IEEE Vehicular Technology Conference, Tokyo, Japan, May 2000.

Xiang Duan, Zhisheng Niu, David Huang, and Dadvid Lee. A dynamic power and rate joint allocation algorithm for mobile multimedia DS-CDMA networks based on utiliity functions. In Proceedings of the IEEE Personal, Indoor and Mobile Radio Communications Conference, September 2002.

Nan Feng, Siun-Chuon Mau, and Narayan B. Mandayam. Pricing and power control for joint network-centric and user-centric radio resource management. IEEE Transactions on Communications, 52(9), 2004.

Felix R. Gantmacher. The Theory of Matrices, Vol. II, Ch. XIII. Chelsea Publishing Company, New York, NY, USA, 1974.

Erik Geijer Lundin and Fredrik Gunnarsson. Uplink load in CDMA cellular radio systems. IEEE Transactions on Vehicular Technology, 2005. To Appear.

Erik Geijer Lundin and Fredrik Gunnarsson. Characterizing uplink load - concepts and algorithms. In Mohsen Guizani, editor, Wireless Communications Systems and Networks, chapter 14, pages 425-441. Kluwer Academic, 2003.

Erik Geijer Lundin and Fredrik Gunnarsson. Using uplink relative path gain related measurements to support uplink resource management. US Patent Application No: $11 / 066,558$.

Erik Geijer Lundin, Fredrik Gunnarsson, and Fredrik Gustafsson. Uplink load estimates in WCDMA with different availability of measurements. In Proceedings of the IEEE Vehicular Technology Conference, Cheju, South Korea, April 2003a. 
Erik Geijer Lundin, Fredrik Gunnarsson, and Fredrik Gustafsson. Adaptive filtering applied to an uplink load estimate in WCDMA. In Proceedings of the IEEE Vehicular Technology Conference, Cheju, South Korea, April 2003b.

Erik Geijer Lundin, Fredrik Gunnarsson, and Fredrik Gustafsson. Uplink load estimation in WCDMA. In Proceedings of the IEEE Wireless Communications and Networking Conference, New Orleans, LA, USA, March 2003c.

Erik Geijer Lundin, Fredrik Gunnarsson, and Fredrik Gustafsson. Robust uplink resource allocation in CDMA cellular radio systems. In Proceedings of the IEEE Conference on Decision and Control, Seville, Spain, December 2005a. To appear.

Erik Geijer Lundin, Fredrik Gunnarsson, and Fredrik Gustafsson. Uplink load and link budget with stochastic noise rise levels in CDMA cellular systems. In RVK05, Linköping, Sweden, June 2005b.

Klein S. Gilhousen, Irwin M. Jacobs, Roberto Padovani, Andrew J. Viterbi, Lindsay A. Weaver Jr., and Charles E. Wheatley. On the capacity of a cellular CDMA system. IEEE Transactions on Vehicular Technology, 40(2), 1991.

Gene H. Golub and Charles F. van Loan. Matrix Computations. The Johns Hopkins University Press, Baltimore, MD, USA, third edition, 1996.

Mikael Gudmundson. Correlation model for shadow fading in mobile radio systems. IEE Electronics Letters, 27(23), November 1991.

Fredrik Gunnarsson. Power Control in Cellular Radio System: Analysis, Design and Estimation. PhD thesis, Linköping University, Linköping, Sweden, April 2000. Linköping Studies in Science and Technology. Dissertations No. 579.

Fredrik Gunnarsson, Erik Geijer Lundin, Gunnar Bark, and Niclas Wiberg. Uplink admission control in WCDMA based on relative load estimates. In IEEE International Conference on Communications, New York, NY, USA, April 2002.

Fredrik Gunnarsson, David Törnqvist, Erik Geijer Lundin, Gunnar Bark, Niclas Wiberg, and Eva Englund. Uplink transmission timing in WCDMA. In IEEE Vehicular Technology Conference, Orlando, FL, USA, October 2003.

Fredrik Gustafsson. Adaptive filtering and change detection. John Wiley \& Sons, LTD, 2000.

Fredrik Gustafsson, Fredrik Gunnarsson, Niclas Bergman, Urban Forssel, Jonas Jansson, Rickard Karlsson, and Per-Johan Nordlund. Particle filters for positioning, navigation and tracking. IEEE Transactions on Signal Processing, 50(2), February 2002.

Hwang Gyung-Ho and Cho Dong-Ho. Dynamic rate control based on interference and transmission power in 3GPP WCDMA system. In Proceedings of the IEEE Vehicular Technology Conference, Boston, MA, USA, September 2000.

Stephen Vaughan Hanly. Capacity and power control in spread spectrum macrodiversity radio networks. IEEE Transactions on Communications, 44(2), 1996. 
Stephen Vaughan Hanly and David N. C. Tse. Power control and capacity of spread spectrum wireless networks. Automatica, 35:1987-2012, 1999.

Bassam Hashem and Elvino Sousa. Reverse link capacity and interference statistics of a fixed-step power-controlled DS/CDMA system under slow multipath fading. IEEE Transactions on Communications, pages 1905-1912, December 1999.

Bassam Hashem and Elvino Sousa. A combined power/rate control scheme for data transmission over a DS/CDMA system. In Proceedings of the IEEE Vehicular Technology Conference, pages 1096-1100, Ottawa, Ontario, Canada, May 1998.

Masayasu Hata. Empirical formula for propagation loss in land mobile radio services. IEEE Transactions on Vehicular Technology, 29(3), 1980.

Jeffrey D. Herdtner and Edwin K.P. Chong. Analysis of a class of distributed asynchronous power control algorithms for cellular wireless systems. IEEE Journal on Selected Areas in Communications, 18(3), March 2000.

Kimmo Hiltunen and Nicola Binucci. WCDMA downlink coverage:interference margin for users located at the cell coverage border. In Proceedings of the IEEE Vehicular Technology Conference, Birmingham, AL, USA, May 2002.

Harri Holma and Janne Laakso. Uplink admission control and soft capacity with MUD in CDMA. In Proceedings of the IEEE Vehicular Technology Conference, Amsterdam, the Netherlands, Sep 1999.

Harri Holma and Antti Toskala. WCDMA for UMTS, Radio Access For Third Generation Mobile Communications. John Wiley \& Sons, LTD, 2000.

Ching-Yao Huang and Roy D. Yates. Call admission in power controlled CDMA systems. In Proceedings of the IEEE Vehicular Technology Conference, Atlanta, GA, USA, May 1996.

Yoshihiro Ishikawa and Narumi Umeda. Capacity design and performance of call admission control in cellular CDMA systems. IEEE Journal on Selected Areas in Communications, 15(8), October 1997.

Ahmad Jalali. On cell breathing in CDMA networks. In Proceedings of the IEEE International Conference on Communications, Atlanta, GA , USA, June 1998.

Riku Jäntti and Seong-Lyun Kim. Transmission rate scheduling for the non-real-time data in a cellular CDMA system. IEEE Communications Letters, 5(5), May 2001.

Tara Javidi. Decentralized rate assignments in a multi-sector CDMA network. In Proceedings of the IEEE Global Telecommunications Conference, Dec 2003.

Thomas Kailath. Linear Systems. Prentice Hall, Englewood Cliffs, NJ, USA, 1980.

Rudolph E. Kalman. A new approach to linear filtering and prediction problems. Journal of Basic Engineering, Transactions of the ASME, 82D:35-45, March 1960. 
Joon Bae Kim and Michael L. Honig. Resource allocation for multiple classes of DSCDMA traffic. IEEE Transactions on Vehicular Technology, 49(2):506-519, May 2000.

Sang Wu Kim and Ye Hoon Lee. Combined rate and power adaptation in DS/CDMA communications over nakagami fading channels. IEEE Transactions on Communications, 48(1), January 2000.

Raymond Knopp and Pierre A. Humblet. Information capacity and power control in single-cell multiuser communications. In Proceedings of the IEEE International Conference on Communications, Seattle, USA, June 1995.

Troels E. Kolding, Frank Frederiksen, and Preben E. Mogensen. Performance aspects of WCDMA systems with high speed downlink packet access (HSDPA). In Proceedings of the IEEE Vehicular Technology Conference, Vancouver, Canada, 2002.

Joy Kuri and Paul Mermelstein. Call admission on the uplink of a CDMA system based on total received power. In Proceedings of the IEEE International Conference on Communications, Vancouver, BC, Canada, June 1999.

Janne Laakso, Riku Jäntti, Mika Rinne, and Oscar Salonaho. Radio resource knapsack packing for WCDMA air interface. In Proceedings of the IEEE Personal, Indoor and Mobile Radio Communications Conference, Boston, MA, USA, Sep 1998.

Jaana Laiho, Achim Wacker, and Tomás Novosad. Radio Network Planning and Optimisation for UMTS. John Wiley \& Sons, LTD, 2002.

Zhuyu Lei, David J. Goodman, and Narayan B. Mandayam. Location-dependent othercell interference and its effect on the uplink capacity of a cellular CDMA system. In Proceedings of the IEEE Vehicular Technology Conference, Houston, TX, USA, May 1999.

Xin Liu, Edwin K. P. Chong, and Ness B. Shroff. Opportunistic transmission scheduling with resource-sharing constraints in wireless networks. IEEE Journal on Selected Areas in Communications, 19(10), October 2001.

Lennart Ljung. System Identification - Theory for the User. Prentice Hall, Englewood Cliffs, NJ, USA., second edition, 1999.

Johan Löfberg. YALMIP : A toolbox for modeling and optimization in MATLAB. In Proceedings of the CACSD Conference, Taipei, Taiwan, 2004. Available from http://control.ee.ethz.ch/ joloef/yalmip.php.

Rohit Nagi, Moses Charikar, and John Cioffi. Minimum outage transmission over fading channels with delayconstraint. In Proceedings of the IEEE International Conference on Communications, pages 282-286, New Orleans, LA, USA, June 2000.

Thomas Neubauer and Ernst Bonek. Impact of the variation in the background noise floor on UMTS system capacity. In Proceedings of the IEEE Vehicular Technology Conference, volume 4, pages 2435-2439, Rhodes, Greece, May 2001. 
Seong-Jun Oh and Kimberly M. Wasserman. Optimal resource allocation in multiservice CDMA networks. IEEE Transactions on Wireless Communications, 2(4), July 2003.

Yoshihisa Okumura, E Ohmori, T. Kawano, and K. Fukuda. Field strength and its variability in VHF and UHF land-mobile radio service. Review of the Electrical Communication Laboratory, 16(9-10), 1968.

James M. Ortega and Werner C. Rheinboldt. Iterative Solution of Nonlinear Equations in Several Variables. Academic Press, London, 1970.

José Outes, Lars Nielsen, Klaus Pedersen, and Preben Mogensen. Multi-cell admission control for UMTS. In Proceedings of the IEEE Vehicular Technology Conference, Rhodes, Greece, May 2001.

Roberto Padovani, B. Butler, and R. Boesel. CDMA digital cellular: field test results. In Proceedings of the IEEE Vehicular Technology Conference, Stockholm, Sweden, June 1994.

Stefan Parkvall, Janne Peisa, Johan Torsner, and Peter Malm. WCDMA enhanced uplink - principles and basic operation. In Proceedings of the IEEE Vehicular Technology Conference, Stockholm, Sweden, May 2005.

Ramin Rezaiifar and Jack Holtzman. Proof of convergence for the distributed optimal rate assignment algorithm. In Proceedings of the IEEE Vehicular Technology Conference, Houston, TX, USA, May 1999.

Branko Ristic, Sanjeev Arulampalam, and Neil Gordon. Beyond the Kalman Filter: Particle Filters for Tracking Applications. Artech House, 2004.

Virgilo Rodriguez, David J. Goodman, and Marantz Zory. Power and data rate assignment for maximal weighted throughput in 3G CDMA: a global solution with two classes of users. In Proceedings of the IEEE Wireless Communications and Networking Conference, March 2004.

Ashwin Sampath, Kumar P. Sarath, and Jack M. Holtzmann. Power control and resource management for a multimedia CDMA wireless system. In Proceedings of the IEEE Personal, Indoor and Mobile Radio Communications Conference, Toronto, Ontario, Canada, September 1995.

Juan Sanchez, Jordi Pérez-Romero, Oriol Sallent, and Ramón Agusti. Mixing conversational and interactive traffic in the UMTS radio access network. In Proc.IEEE 4th International Workshop on Mobile and Wireless Communications Network, 2002, Stockholm, Sweden, September 2002.

Stanley F. Schmidt. Application of state-space methods to navigation problems. Advances in Control Systems, C.T. Leondes, Ed., Academic Press, 3:293-340, 1966.

Stuart C. Schwartz and Yu-Shuan Yeh. On the distribution function and moments of power sums with log-normal components. The Bell System Technical Journal, 61(7), 1982. 
Kari Sipilä, Jaana Laiho-Steffens, Achim Wacker, and Mika Jäsberg. Modeling the impact of the fast power control on the WCDMA uplink. In Proceedings of the IEEE Vehicular Technology Conference, Houston, Texas, May 1999.

Bernard Sklar. Rayleigh fading channels in mobile digital communication systems. IEEE Communications Magazine, 35(7), 1997.

Sigurd Skogestad and Ian Postlethwaite. Multivariable Feedback Control. John Wiley \& Sons, LTD, 1996.

Gerald L. Smith, Stanley F. Schmidt, and Leonard A. McGee. Application of statistical filter theory to the optimal estimation of position and velocity on board a circumlunar vechicle. Technical report, NASA TR T-35, National Aeronautics and Space Administration, 1962.

Peter Stevens. Operator design and planning issues for UMTS networks. In IEE Colloquium on UMTS - The R\&D Challenges (Ref. No. 1998/496), London, UK, November 1998.

Bogdan Timus and Jonas Pettersson. Uplink admission control for conversational services using information from many cells. In Proceedings of the IEEE Vehicular Technology Conference, Rhodes, Greece, May 2001.

Kim C. Toh, Michael J. Todd, and Reha H. Tütüncü. SDPT3 - a MATLAB software package for semidefinite programming, version 3.02, September 2001.

David Törnqvist, Erik Geijer Lundin, Fredrik Gunnarsson, and Fredrik Gustafsson. Transmission timing - a control approach to distributed uplink scheduling in WCDMA. In Proceedings of the American Control Conference, Boston, MA, USA, June 2004.

Sennur Ulukus and Larry J. Greenstein. Throughput maximization in CDMA uplinks using adaptive spreading and power control. In Proceedings of the Symposium on Spread Spectrum Techniques and Applicatoins, September 2000.

John M. Wozencraft and Irwin Mark Jacobs. Principles of Communication Engineering. John Wiley \& Sons, LTD, 1965.

Roy D. Yates. A framework for uplink power control in cellular radio systems. IEEE Journal on Selected Areas in Communications, 13(7), September 1995.

Wang Ying, Zhang Jingmei, Wang Weidong, and Zhang Ping. Call admission control in hierarchi cell structure. In Proceedings of the IEEE Vehicular Technology Conference, Birmingham, AL, USA, May 2002.

Jens Zander. Transmitter power control for co-channel interference management in cellular radio systems. In Proc. WINLAB Workshop, New Brunswick, NJ, USA, October 1993.

Jens Zander, Seong-Lyun Kim, Magnus Almgren, and Olav Queseth. Arthech House, 2001. 
Qinqing Zhang and On-Ching Yue. UMTS air interface voice/data capacity - part 1:reverse link analysis. In Proceedings of the IEEE Vehicular Technology Conference, Rhodes, Greece, May 2001.

Kemin Zhou, John C. Doyle, and Keith Glover. Robust and Optimal Control. Prentice Hall, Upper Saddle River, NJ, USA, 1995. 


\section{Index}

3GPP, 37

active set, 32

admission control, 35

background noise power ratio, 114

base station, 30

capacity, 33

capacity limited system, 121

carrier-to-total-interference ratio, 31

CDMA, 29

cell, 30

cellular radio network, 30

channel switching, 36

chip rate, 29

CIR, 28, 31

congestion control, 36

connectivity, 82

strict, 82

correlation distance, 26

coverage limited system, 121

CTIR, 31

CUSUM, 134

downlink, 30

enhanced uplink, 38 fast fading, 27

FDMA, 29

feasibility, 82

feasibility relative load, 85

grade of service (GoS), 33

handover, 31

soft, 32

softer, 38

hard capacity, 42

intercell-to-intracell-interference factor, 44 interference

intercell, 33, 43

intracell, 43

total, 31

interference limited system, 41

Kalman filter

extended, 133

linear, 131

link admission, 36

link matrix, 82

maximum ratio combining, 32

multipath fading, 27 
noise limited system, 41

noise rise, 40

noise rise relative load, 41

non-orthogonal signals, 29

observability, 144

orthogonal signals, 29

packet scheduling, 36

particle filter, 134

party effect, 35

path loss, 26

pole capacity, 41

power control, 31, 34

power gain, 26

power gain matrix, 30

processing gain, 29

propagation exponent, 26

Quality of Service (QoS), 33

radio network controller (RNC), 37

radio resource management (RRM), 34

rake, 27

relative power gain matrix, 83

RRM, 34

selection combining, 32

self interference factor, 32

shadow fading, 26

SIR, 28

soft capacity, 42

soft resource, 43

spreading code, 29

system feasibility, 82

system matrix, 83

system noise rise relative load, 84

TDMA, 29

total interference power, 31

TX increase, 64

uplink, 30

WCDMA, 37 


\section{PhD Dissertations \\ Division of Automatic Control \\ Linköpings universitet}

M. Millnert: Identification and control of systems subject to abrupt changes. Thesis No. 82, 1982. ISBN 91-7372-542-0.

A. J. M. van Overbeek: On-line structure selection for the identification of multivariable systems. Thesis No. 86, 1982. ISBN 91-7372-586-2.

B. Bengtsson: On some control problems for queues. Thesis No. 87, 1982. ISBN 91-7372-593-5.

S. Ljung: Fast algorithms for integral equations and least squares identification problems. Thesis No. 93, 1983. ISBN 91-7372-641-9.

H. Jonson: A Newton method for solving non-linear optimal control problems with general constraints. Thesis No. 104, 1983. ISBN 91-7372-718-0.

E. Trulsson: Adaptive control based on explicit criterion minimization. Thesis No. 106, 1983. ISBN 91-7372-728-8.

K. Nordström: Uncertainty, robustness and sensitivity reduction in the design of single input control systems. Thesis No. 162, 1987. ISBN 91-7870-170-8.

B. Wahlberg: On the identification and approximation of linear systems. Thesis No. 163, 1987. ISBN 91-7870-175-9.

S. Gunnarsson: Frequency domain aspects of modeling and control in adaptive systems. Thesis No. 194, 1988. ISBN 91-7870-380-8.

A. Isaksson: On system identification in one and two dimensions with signal processing applications. Thesis No. 196, 1988. ISBN 91-7870-383-2.

M. Viberg: Subspace fitting concepts in sensor array processing. Thesis No. 217, 1989. ISBN 917870-529-0.

K. Forsman: Constructive commutative algebra in nonlinear control theory. Thesis No. 261, 1991. ISBN 91-7870-827-3.

F. Gustafsson: Estimation of discrete parameters in linear systems. Thesis No. 271, 1992. ISBN 91-7870-876-1.

P. Nagy: Tools for knowledge-based signal processing with applications to system identification. Thesis No. 280, 1992. ISBN 91-7870-962-8.

T. Svensson: Mathematical tools and software for analysis and design of nonlinear control systems. Thesis No. 285, 1992. ISBN 91-7870-989-X.

S. Andersson: On dimension reduction in sensor array signal processing. Thesis No. 290, 1992. ISBN 91-7871-015-4.

H. Hjalmarsson: Aspects on incomplete modeling in system identification. Thesis No. 298, 1993. ISBN 91-7871-070-7.

I. Klein: Automatic synthesis of sequential control schemes. Thesis No. 305, 1993. ISBN 917871-090-1.

J.-E. Strömberg: A mode switching modelling philosophy. Thesis No. 353, 1994. ISBN 91-7871430-3.

K. Wang Chen: Transformation and symbolic calculations in filtering and control. Thesis No. 361, 1994. ISBN 91-7871-467-2.

T. McKelvey: Identification of state-space models from time and frequency data. Thesis No. 380, 1995. ISBN 91-7871-531-8.

J. Sjöberg: Non-linear system identification with neural networks. Thesis No. 381, 1995. ISBN 917871-534-2.

R. Germundsson: Symbolic systems - theory, computation and applications. Thesis No. 389, 1995. ISBN 91-7871-578-4.

P. Pucar: Modeling and segmentation using multiple models. Thesis No. 405, 1995. ISBN 917871-627-6.

H. Fortell: Algebraic approaches to normal forms and zero dynamics. Thesis No. 407, 1995. ISBN 91-7871-629-2. 
A. Helmersson: Methods for robust gain scheduling. Thesis No. 406, 1995. ISBN 91-7871-628-4. P. Lindskog: Methods, algorithms and tools for system identification based on prior knowledge. Thesis No. 436, 1996. ISBN 91-7871-424-8.

J. Gunnarsson: Symbolic methods and tools for discrete event dynamic systems. Thesis No. 477, 1997. ISBN 91-7871-917-8.

M. Jirstrand: Constructive methods for inequality constraints in control. Thesis No. 527, 1998. ISBN 91-7219-187-2.

U. Forssell: Closed-loop identification: Methods, theory, and applications. Thesis No. 566, 1999. ISBN 91-7219-432-4.

A. Stenman: Model on demand: Algorithms, analysis and applications. Thesis No. 571, 1999. ISBN 91-7219-450-2.

N. Bergman: Recursive Bayesian estimation: Navigation and tracking applications. Thesis No. 579, 1999. ISBN 91-7219-473-1.

K. Edström: Switched bond graphs: Simulation and analysis. Thesis No. 586, 1999. ISBN 917219-493-6.

M. Larsson: Behavioral and structural model based approaches to discrete diagnosis. Thesis No. 608, 1999. ISBN 91-7219-615-5.

F. Gunnarsson: Power control in cellular radio systems: Analysis, design and estimation. Thesis No. 623, 2000. ISBN 91-7219-689-0.

V. Einarsson: Model checking methods for mode switching systems. Thesis No. 652, 2000. ISBN 91-7219-836-2.

M. Norrlöf: Iterative learning control: Analysis, design, and experiments. Thesis No. 653, 2000. ISBN 91-7219-837-0.

F. Tjärnström: Variance expressions and model reduction in system identification. Thesis No. 730, 2002. ISBN 91-7373-253-2.

J. Löfberg: Minimax approaches to robust model predictive control. Thesis No. 812, 2003. ISBN 91-7373-622-8.

J. Roll: Local and piecewise affine approaches to system identification. Thesis No. 802, 2003. ISBN 91-7373-608-2.

J. Elbornsson: Analysis, estimation and compensation of mismatch effects in A/D converters. Thesis No. 811, 2003. ISBN 91-7373-621-X.

O. Härkegård: Backstepping and control allocation with applications to flight control. Thesis No. 820, 2003. ISBN 91-7373-647-3.

R. Wallin: Optimization algorithms for system analysis and identification. Thesis No. 919, 2004. ISBN 91-85297-19-4.

D. Lindgren: Projection methods for classification and identification. Thesis No. 915, 2005. ISBN 91-85297-06-2.

R. Karlsson: Particle Filtering for Positioning and Tracking Applications. Thesis No. 924, 2005. ISBN 91-85297-34-8.

J. Jansson: Collision Avoidance Theory with Applications to Automotive Collision Mitigation. Thesis No. 950, 2005. ISBN 91-85299-45-6. 